\title{
HETEROLEPTIC ALUMINUM HYDRIDE COMPOUNDS: \\ SYNTHESIS, THERMOLYSIS, AND APPLICATION \\ IN ALUMINUM METAL THIN FILM DEPOSITION \\ THROUGH CHEMICAL VAPOUR METHODS
}

by

Julie R. Delahunt B.Sc.

\begin{abstract}
A thesis submitted to the Faculty of Graduate Studies and Research in partial fulfillment of the requirements for the degree of

Master of Science
\end{abstract}

to

The Faculty of Graduate Studies and Research

Department of Chemistry

Carleton University

Ottawa, Ontario

Canada

(C) 2010, Julie R. Delahunt 
Library and Archives

Canada

Published Heritage

Branch

395 Wellington Street

Ottawa ON K1A ON4

Canada
Bibliothèque et

Archives Canada

Direction du

Patrimoine de l'édition

395 , rue Wellington

Ottawa ON K1A ON4

Canada
Your file Votre référence

ISBN: 978-0-494-71580-2

Our file Notre référence

ISBN: 978-0-494-71580-2
NOTICE:

The author has granted a nonexclusive license allowing Library and Archives Canada to reproduce, publish, archive, preserve, conserve, communicate to the public by telecommunication or on the Internet, loan, distribute and sell theses worldwide, for commercial or noncommercial purposes, in microform, paper, electronic and/or any other formats.

The author retains copyright ownership and moral rights in this thesis. Neither the thesis nor substantial extracts from it may be printed or otherwise reproduced without the author's permission.
AVIS:

L'auteur a accordé une licence non exclusive permettant à la Bibliothèque et Archives Canada de reproduire, publier, archiver, sauvegarder, conserver, transmettre au public par télécommunication ou par l'Internet, prêter, distribuer et vendre des thèses partout dans le monde, à des fins commerciales ou autres, sur support microforme, papier, électronique et/ou autres formats.

L'auteur conserve la propriété du droit d'auteur et des droits moraux qui protège cette thèse. $\mathrm{Ni}$ la thèse ni des extraits substantiels de celle-ci ne doivent être imprimés ou autrement reproduits sans son autorisation.
In compliance with the Canadian Privacy Act some supporting forms may have been removed from this thesis.

While these forms may be included in the document page count, their removal does not represent any loss of content from the thesis.
Conformément à la loi canadienne sur la protection de la vie privée, quelques formulaires secondaires ont été enlevés de cette thèse.

Bien que ces formulaires aient inclus dans la pagination, il n'y aura aucun contenu manquant. 


\section{ABSTRACT}

The synthesis of five 5-coordinate amidinate hydrido aluminum compounds with general structure $\left[\mathrm{R}\left(\mathrm{NC}\left(\mathrm{N}^{\mathrm{i}} \mathrm{Pr}\right)_{2}\right]_{2} \mathrm{AlH}\right.$ using ligand exchange and hydrogen elimination methods. Compounds 1-5, with $\mathrm{R}=\mathrm{NMe}_{2}, \mathrm{NEt}_{2}, \mathrm{~N}^{\prime} \mathrm{PrH}, \mathrm{N}^{\mathrm{i}} \mathrm{Pr}_{2}$, and $\mathrm{Me}$, were isolated with respective yields of $85 \%, 86 \%, 93 \%, 78 \%$, and $16 \%$. The five compounds were investigated as potential precursors for atomic layer deposition (ALD): structural and thermal characteristics were studied using melting point, sublimation temperature, single crystal X-ray diffraction (scXRD), thermogravimetric analysis (TGA), ${ }^{1} \mathrm{H}$ NMR studies, and time-of-flight mass spectroscopy (TOFMS). Pulsed chemical vapour deposition (CVD) experiments were performed with compound 3 and forming gas $\left(5 \% \mathrm{H}_{2}\right.$ in $\left.\mathrm{N}_{2}\right)$. Deposited films were characterized using scanning electron microscopy (SEM) and powder X-ray diffraction ( $\mathrm{PXRD)}$ and determined to be aluminum metal in a cubic closest packed (ccp) arrangement. 


\section{ACKNOWLEGMENTS}

I would like to express my gratitude towards my supervisor, Dr. Seán Barry, for allowing me to join his research group during my undergrad, and for inviting me to continue my research in pursuit of a MSc degree. Seán is an excellent supervisor providing each of his students with as much advice, or as much independence, as each individual would desire. His leadership and guidance was invaluable over the years- discussions over morning and afternoon coffee will be greatly missed.

To all of the Barry lab group members, past and present: thank you for providing an excellent work environment. Thank you to past members who helped to establish the lab, whose research provided a great foundation to expand upon. To those whose research terms overlapped with mine, thank you all for excellent advice and discussions, but most of all for friendship and memories.

A special thanks to those who assisted in the characterization contained in this thesis: scXRD was solved by Dr. Glenn Yap at the University of Delaware, TOF-MS was performed by Jason Coyle, under the supervision of Dr. Jens Müller at the University of Saskatchewan, pXRD was performed by various members of the Sundararajan group at Carleton University, and SEM training was provided by Dr. Jianqun Wang at Carleton University.

I would especially like to thank my family, whose love and support have been essential to the completion of this degree. Finally to Peter, whose love has done more for me than he will ever know, couldn't have done it without him. 


\section{TABLE OF CONTENTS:}

ABSTRACT

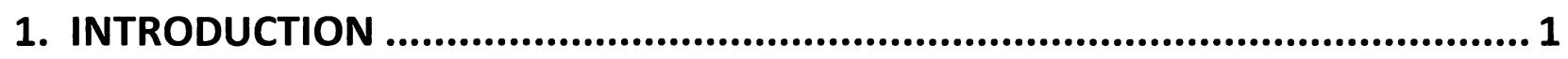

1.1 Aluminum

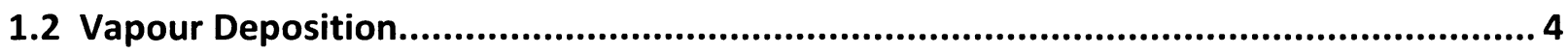

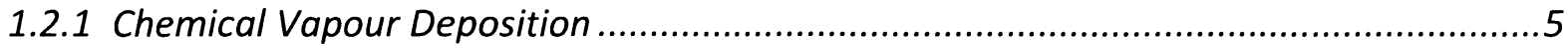

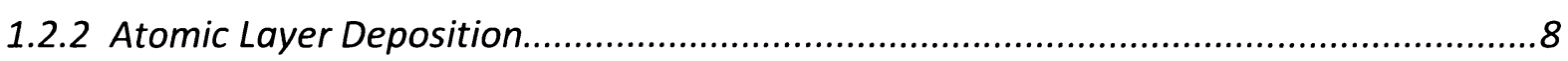

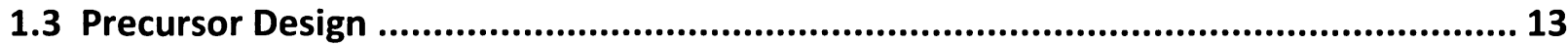

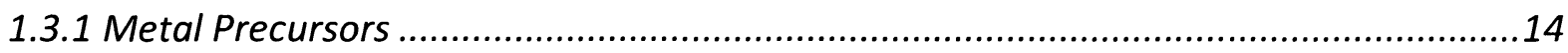

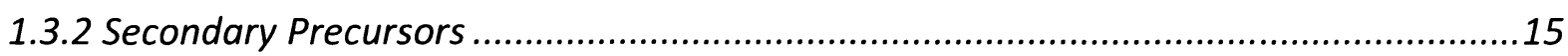

1.3.3 Amidinates and Guanidinates ..........................................................................18

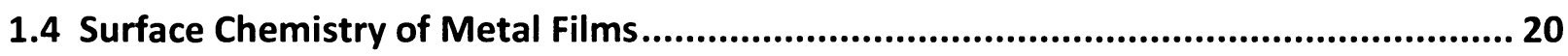

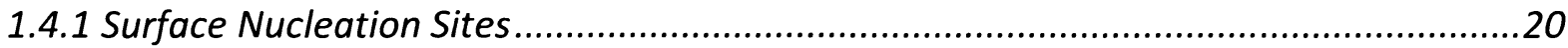

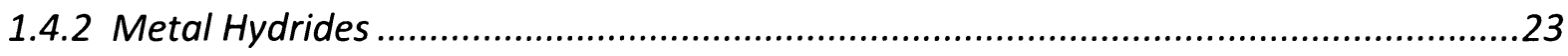

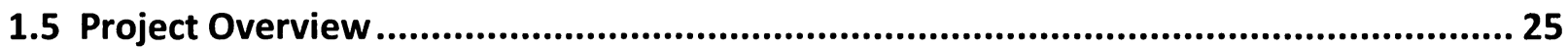

2. HETEROLEPTIC ALUMINUM COMPOUNDS ............................................ 27

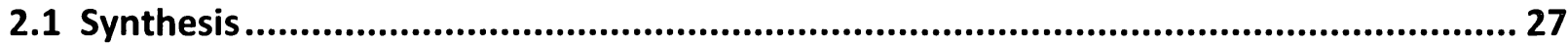

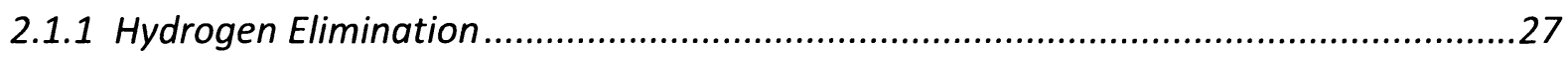

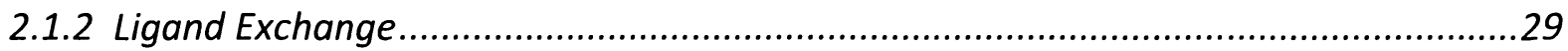

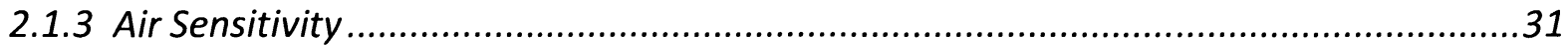




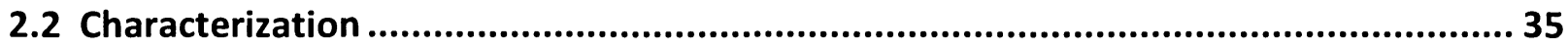

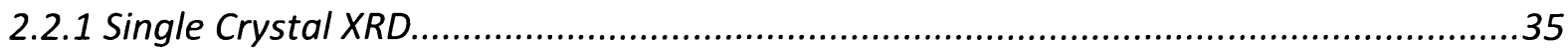

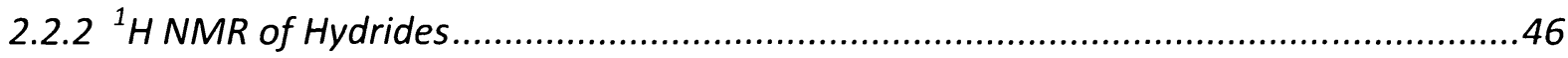

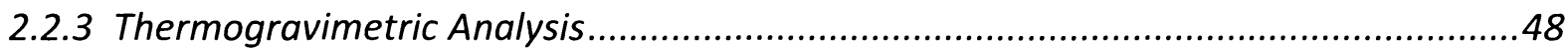

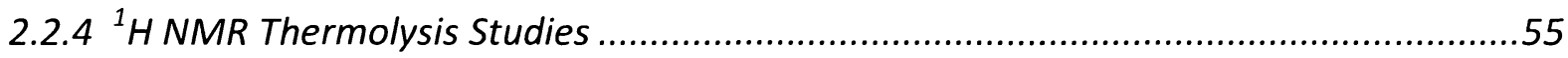

2.2.5 Time-of-Flight Mass Spectrometry ........................................................................62

3. ALUMINUM METAL DEPOSITION .......................................................67 67

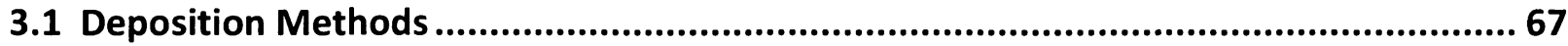

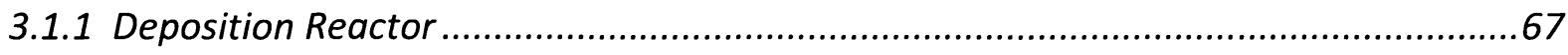

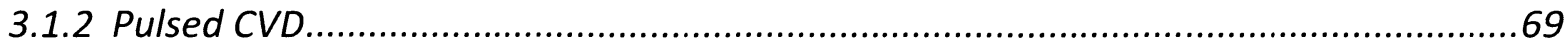

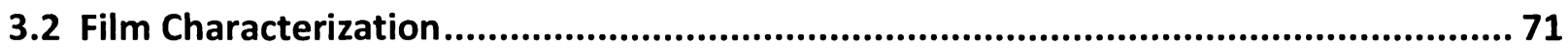

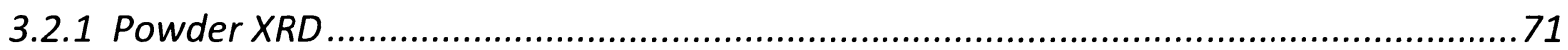

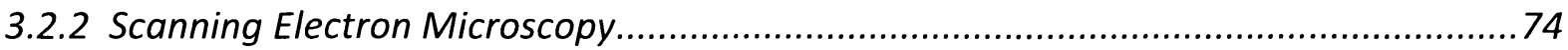

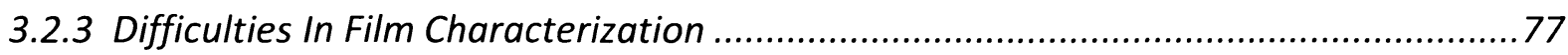

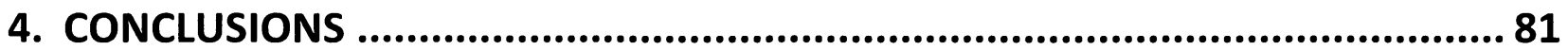

4.1 Synthesis and Characterization of Heteroleptic Aluminum Compounds ....................... 81

4.2 Aluminum Metal Deposition and Film Characterization............................................... 83

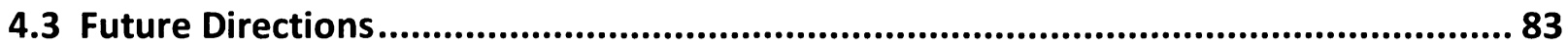

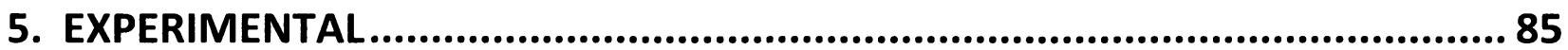

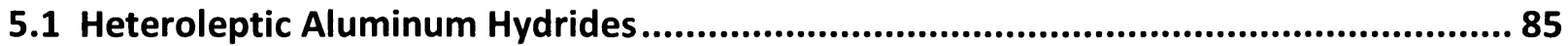

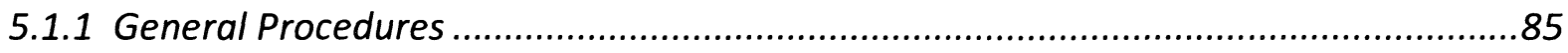

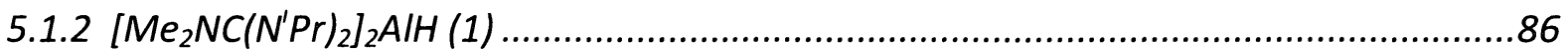

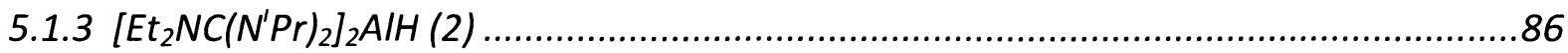

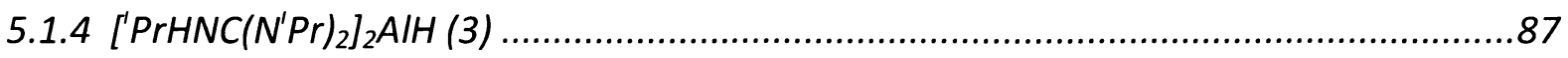

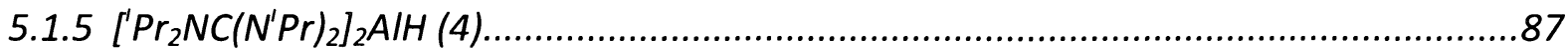

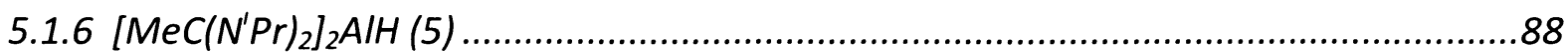

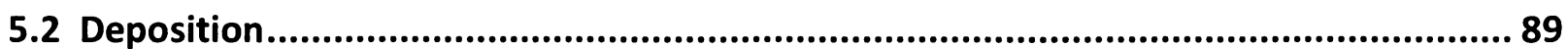

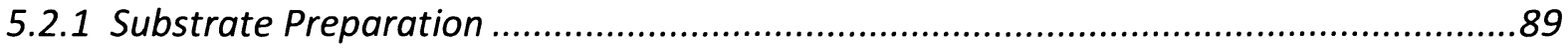




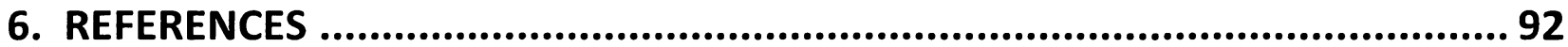

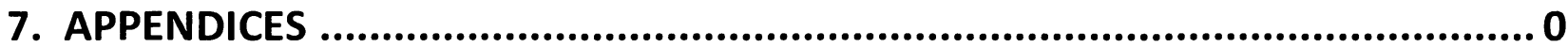




\section{LIST OF FIGURES}

Figure 1. Decreasing thickness of copper interconnects within dynamic random access memory (DRAM) capacitor dielectrics ${ }^{(3 ; 4)}$ 1

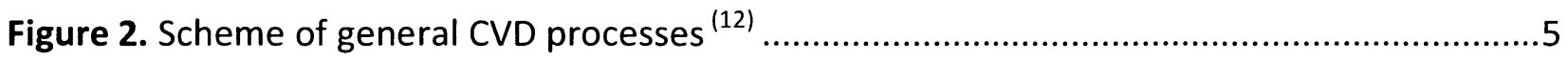

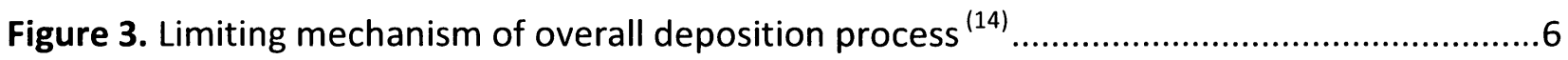

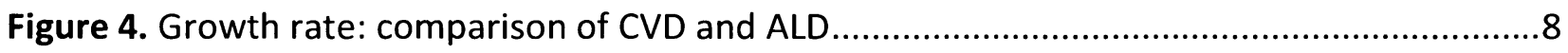

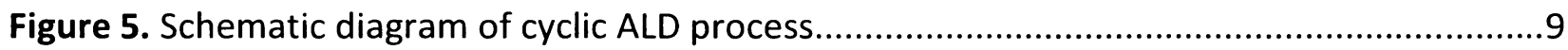

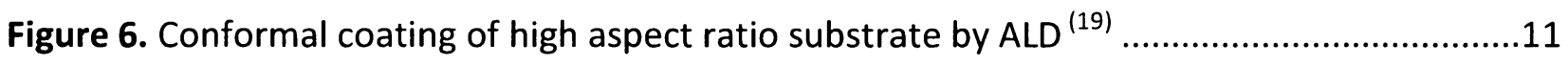

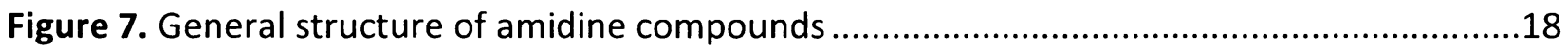

Figure 8. Resonance structures of A) amidinate ligands, and B) guanidinate ligands.................19

Figure 9. Amidinate and guanidinate coordination to aluminum (iii) metal centre.....................20

Figure 10. Hydroxyl density on high surface area silica $A$ ) general trend based on $\mathrm{Si}^{29} \mathrm{NMR}$ and

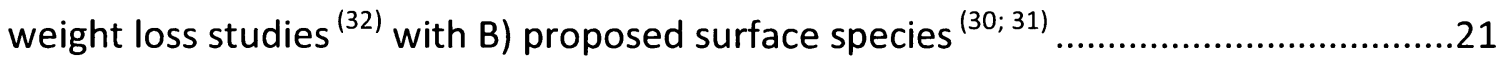

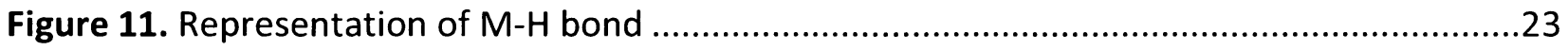

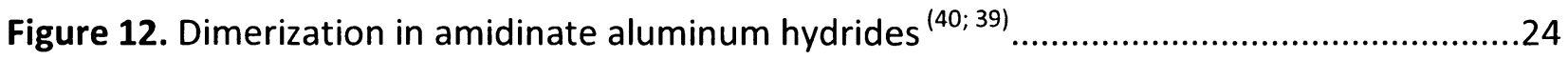

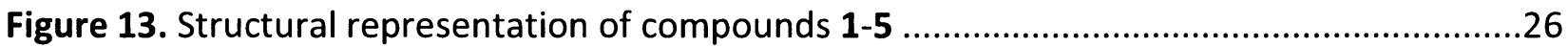

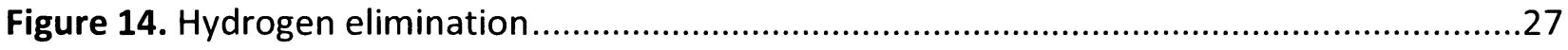

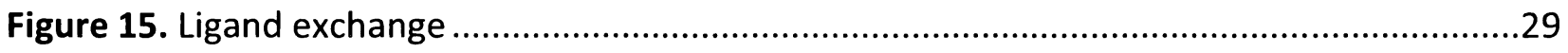

Figure 16. Needle-like crystal formations of triisopropyl guanidine resultant from air assisted

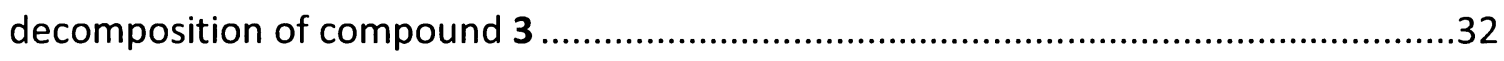

Figure 17. Aluminum metal formations from compound 5 in ambient conditions.....................33

Figure 18. Crystal structure of compound 1 A) view normal to Al-H1, B) view along Al-H1, and C) selected bond lengths and angles..............................................................................38 
Figure 19. Crystal structure of compound 2 A) view normal to Al-H1, B) view along Al-H1, and C) selected bond lengths and angles. 39

Figure 20. Crystal structure of compound 4 A) view normal to Al-H1, B) view along Al-H1, and C) selected bond lengths and angles. .40

Figure 21. Crystal structure of compound 5 A) view normal to Al-H1, B) view along Al-H1, and C) selected bond lengths and angles

Figure 22. $\tau$ parameter for geometric description of 5-coordinate compounds ${ }^{(51)}$ .42

Figure 23. Berry pseudorotation for 5-coordinate compounds. .45

Figure 24. Hydride peak in baseline of ${ }^{1} H$ NMR for A) compound 1 , B) compound 2, C) compound 3, D) compound 4, and E) compound 5

Figure 25. Thermogravimetric analysis of compounds 1-5 .49

Figure 26. Thermogravimetric analysis of compound $\mathbf{1}$ with derivative weight curve 51

Figure 27. Thermogravimetric analysis of compound $\mathbf{2}$ with derivative weight curve .51

Figure 28. Thermogravimetric analysis of compound 3 with derivative weight curve .52

Figure 29. Thermogravimetric analysis of compound 4 with derivative weight curve .52

Figure 30. Thermogravimetric analysis of compound 5 with derivative weight curve .53

Figure 31. ${ }^{1} \mathrm{H}$ NMR Boilup data collected for the decomposition of A) compounds $\mathbf{1}$ and $\mathbf{2}$ at $56^{\circ} \mathrm{C}$, and $\mathrm{B}$ ) compounds 3,4 , and 5 at $65^{\circ} \mathrm{C}$. .58

Figure 32. Decomposition of compounds $1(\boldsymbol{\square})$ at $56^{\circ} \mathrm{C}$ into products $\left[\mathrm{Me}_{2} \mathrm{NC}\left(\mathrm{N}^{\prime} \mathrm{Pr}\right)_{2}\right] \mathrm{Al}\left(\mathrm{NMe}_{2}\right)_{2}$ $(--),\left[\mathrm{HC}\left(\mathrm{N}^{\prime} \mathrm{Pr}\right)_{2}\right]_{2} \mathrm{AlH}(\ldots)$ and $\left[\mathrm{HC}\left(\mathrm{N}^{\prime} \mathrm{Pr}\right)_{2}\right]_{3} \mathrm{Al}(--)$ 60

Figure 33. Decomposition products of compounds 1 at $56^{\circ} \mathrm{C}$ 60

Figure 34. Proposed decomposition mechanism of guanidinate aluminum hydrides based on thermolysis of compound 1 61

Figure 35. Electron impact mass spectrum of compound $\mathbf{3}$ at room temperature. .63 
Figure 36. Temperature dependence of selected mass fragments measured by TOF-MS during thermolysis compound 3 . .64

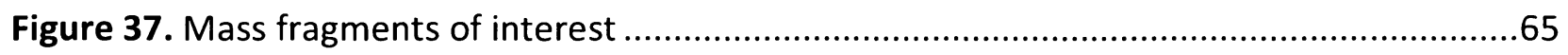

Figure 38. Schematic diagram of custom made hot-walled ALD reactor .68

Figure 39. Pulse program used for A) preliminary experiments and B) bulk of deposition experiments. .69

Figure 40. XRD powder pattern of film deposited with compound 3, overlaid with literature powder pattern for ccp aluminum metal ${ }^{(54) \ddagger}$ .72

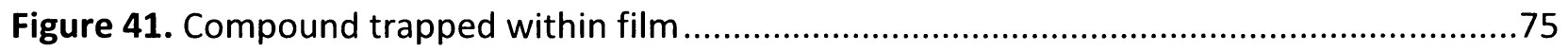

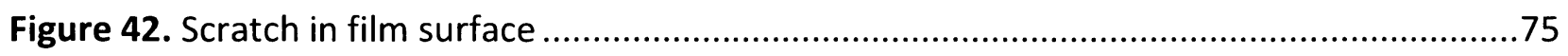

Figure 43. Scotch tape test of aluminum metal film ..................................................76

Figure 44. Energy dispersive spectroscopy of aluminum metal film ..................................79

Figure 45. Edge of deposited film on Si wafer with A) $10^{\circ}$ tilt, $2000 \mathrm{x}$, B) $10^{\circ}$ tilt, $900 \mathrm{x}$, C) $10^{\circ}$ tilt, $600 x$, and D) $5^{\circ}$ tilt, $518 x$ 


\section{LIST OF TABLES}

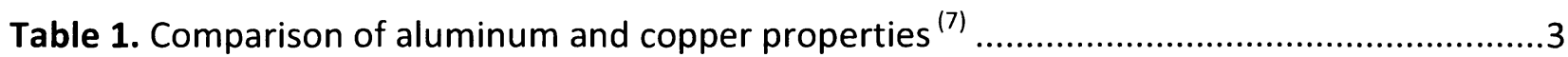

Table 2. Selected preliminary characterization data for compounds 1-5................................35

Table 3. Selected crystal data and structural refinement parameters for compounds

$1,2,4$, and 5 .36

Table 4. Determination of the $\tau$ parameter

Table 5. Structural perturbations experienced by compounds 1, 2, 4 and 5 .43

Table 6. Comparison of hydride bond length and chemical shift ....

Table 7. Thermal characteristics of compounds 1-5 .50

Table 8. Kinetic data collected for compounds 1-5 .58

Table 9. Comparison of experimental and literature pXRD data ${ }^{(46) \ddagger}$ .73 


\title{
LIST OF ABBREVIATIONS
}

\author{
ALD - Atomic Layer Deposition \\ ALE - Atomic Layer Epitaxy \\ CVD - Chemical Vapour Deposition \\ EDS - Electron Diffraction Spectrometry \\ 'Pr-CDI - diisopropyl carbodiimide \\ MOCVD - Metallic Organic Chemical Vapour Deposition \\ MP - Melting Point \\ MS - Mass Spectrometry \\ NMR - Nuclear Magnetic Resonance \\ PEALD - Plasma Enhanced Atomic Layer Deposition \\ pXRD - powder X-ray Diffraction \\ SCXRD - single crystal X-ray Diffraction \\ SEM - Scanning Electron Microscopy \\ TGA - Thermogravimetric Analysis \\ TMA - trimethylaluminum \\ TOF-MS - Time-of-Flight Mass Spectroscopy
}




\section{LIST OF APPENDICES}

\section{Appendix A: Structural Determination by Single Crystal XRD}

Selected crystal data and structural refinement parameters for compounds $1,2,4$, and $5 \ldots . . . A-1$

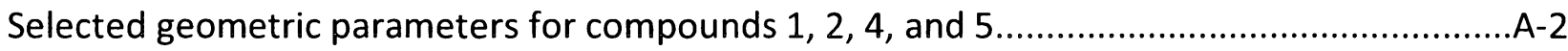

Compound 1: $\left[\mathrm{Me}_{2} \mathrm{NC}\left(\mathrm{N}^{\prime} \mathrm{Pr}\right)_{2}\right]_{2} \mathrm{AlH}$

-Selected structural views

-Full crystallographic information '.cif'.

Compound 2: $\left[\mathrm{Et}_{2} \mathrm{NC}\left(\mathrm{N}^{\prime} \mathrm{Pr}\right)_{2}\right]_{2} \mathrm{AlH}$

-Selected structural views

-Full crystallographic information '.cif'. A-17 to $A-26$

Compound 4: ['Pr $\left.{ }_{2} \mathrm{NC}\left(\mathrm{N}^{\prime} \mathrm{Pr}\right)_{2}\right]_{2} \mathrm{AlH}$

-Selected structural views.

-Full crystallographic information '.cif' A-28 to $A-44$

Compound 5: $\left[\mathrm{MeC}\left(\mathrm{N}^{\prime} \mathrm{Pr}\right)_{2}\right]_{2} \mathrm{AlH}$

-Selected structural views. A-45

-Full crystallographic information '.cif'. A-46 to $A-52$

\section{Appendix B: ${ }^{1} \mathrm{H}$ NMR Spectra for Thermolysis Studies}

Summary of boil up data....

Chemical shift of hydride for compound 1-5.

Compound 1: $\left[\mathrm{Me}_{2} \mathrm{NC}\left(\mathrm{N}^{\prime} \mathrm{Pr}\right)_{2}\right]_{2} \mathrm{AlH}$

- at room temperature.

- at $56^{\circ} \mathrm{C}$ after 310 hours 
Compound 2: $\left[\mathrm{Et}_{2} \mathrm{NC}\left(\mathrm{N}^{\prime} \mathrm{Pr}\right)_{2}\right]_{2} \mathrm{AlH}$

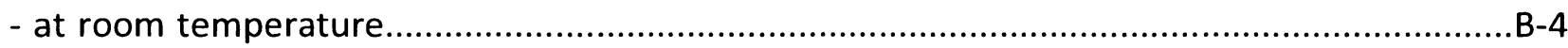

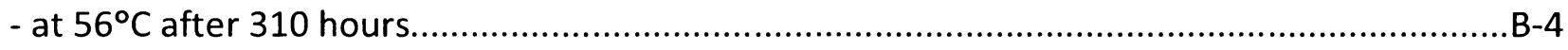

Compound 3: ['PrHNC(N'Pr $\left.)_{2}\right]_{2} \mathrm{AlH}$

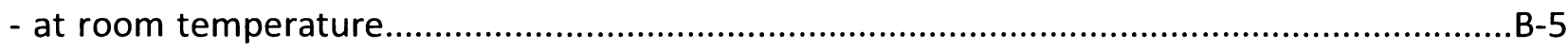

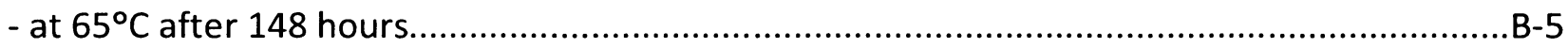

Compound 4: ['Pr $\left.2 \mathrm{NC}\left(\mathrm{N}^{\prime} \mathrm{Pr}\right)_{2}\right]_{2} \mathrm{AlH}$

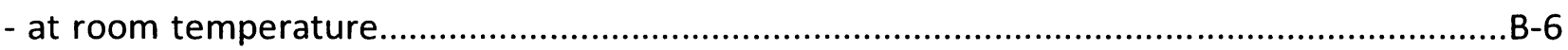

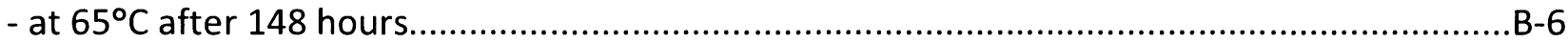

Compound 5: $\left[\mathrm{MeC}\left(\mathrm{N}^{\prime} \mathrm{Pr}\right)_{2}\right]_{2} \mathrm{AlH}$

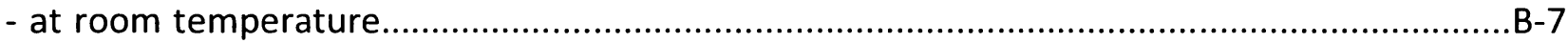

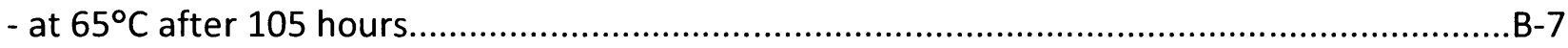

\section{Appendix C: Deposition Parameters}

Schematic diagram of hot-walled ALD reactor......................................................................

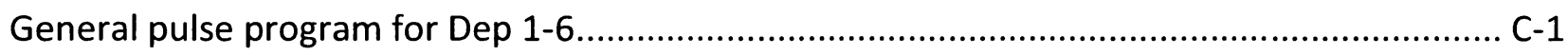

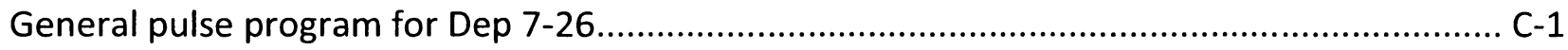

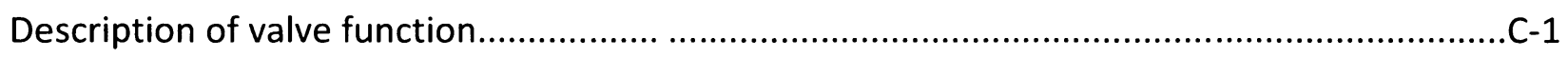

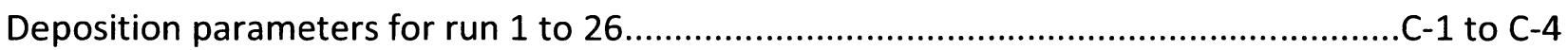




\section{INTRODUCTION}

Based on information collected between 1959 and 1965, Dr. Gordon E. Moore predicted that the number of components included per integrated function would increase linearly; doubling each year. ${ }^{(1)}$ This prediction, known as "Moore's Law", has been remarkably accurate with the only modification being to extend the doubling period to every two years. ${ }^{(2) \ddagger}$ Alongside this trend, we have seen a rapid downsizing in electronic components: traditional electronics became microelectronics, and microelectronics are becoming nanoelectronics. With this trend, each component in a simple circuit requires reinvention; from the materials incorporated into the system, to logistics of how to position and connect various components.

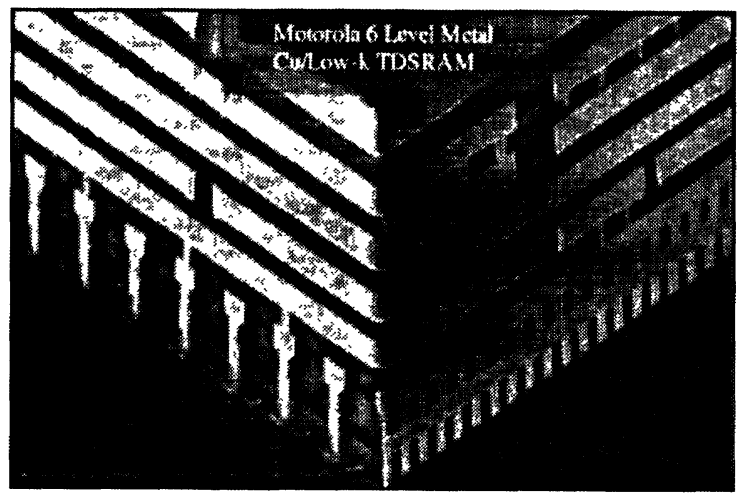

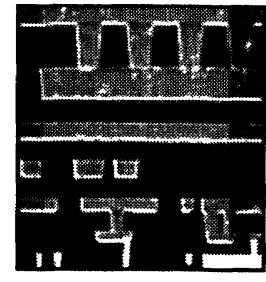

1997

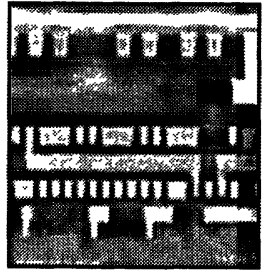

2002

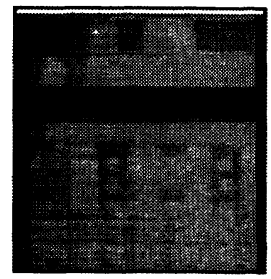

2004

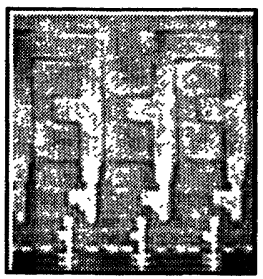

2006

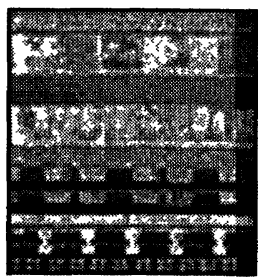

2008

$250 \mathrm{~nm} \mathrm{Cu} \quad 130 \mathrm{~nm} \mathrm{Cu} \quad 90 \mathrm{~nm} \mathrm{Cu} \quad 65 \mathrm{~nm} \mathrm{Cu} \quad 45 \mathrm{~nm} \mathrm{Cu}$

Figure 1. Decreasing thickness of copper interconnects within dynamic random access memory (DRAM) capacitor dielectrics ${ }^{(3 ; 4)}$

\footnotetext{
"Now the one that gets quoted is doubling every 18 months... I think it was Dave House, who used to work here at Intel, did that, he decided that the complexity was doubling every two years and the transistors were getting faster, that computer performance was going to double every 18 months... but that's what got on Intel's Website... and everything else. I never suid 18 months that's the way it often gets quoted." - Dr. Gordon Moore 21
} 
Advances in nanotechnology research have provided answers to these registration issues. ${ }^{(5,4,6)}$ The challenge now is to produce conductive interconnect materials with a high level of control. Herein, the use of aluminum metal thin films grown by thermal atomic layer deposition (ALD) is proposed as a solution to the interconnect problem. The synthesis and characterization of five amidinate aluminum hydride compounds is presented and their suitability for use as precursors for ALD is explored. Thermal ALD of aluminum metal thin films using these compounds in conjunction with a forming gas reducing agent is attempted, and characterization of aluminum metal films grown by a pulsed chemical vapour deposition (CVD) method are presented.

\subsection{Aluminum}

Often, interconnects in electronic devices are made out of copper due to copper's excellent thermal conductivity $\left(4.01 \mathrm{~W} \cdot \mathrm{cm}^{-1} \cdot \mathrm{K}^{-1}\right) \cdot{ }^{(7)}$ However, as film thickness demands approach $5-10 \mathrm{~nm}$ thick, copper by any known deposition process will result in island growth introducing grain boundaries, thus increasing resistivity. Figure 1 demonstrates the decrease in copper interconnect thickness between 1997 and 2008. ${ }^{(4)}$ The denoted thickness refers to the width at the bottom of the copper segment. The total thickness of the deposited film is shown in the figure as the height of the copper segment (not quantified). Currently, there remains a lot of interest in the use of copper for interconnects and a lot of research into ways of solving this problem continue. ${ }^{(4)}$ In the mean time, researchers are also turning to alternative interconnect materials and the development of suitable deposition processes.

Aluminum is the most abundant element in the Earth's crust making up for approximately $8 \%$ of the crusts mass. Thus, the availability of this resource makes aluminum 
very appealing in terms of scale up production and inclusion into electronic devices, while at the same time being economically feasible. Table 1 presents some properties of aluminum in relation to copper as bulk materials.

Aluminum is a versatile main group element, possessing many characteristics of transition metals, but does not possess d-block electrons. Aluminum can adopt $+1,+2$, and +3 oxidation states, but is most commonly found as +3 . Aluminum is known as a Lewis acidic, electron dense metal, and as such tends to possess strong interactions with electron donors such as oxides and nitrides. Aluminum's ability to accept electron density allows a +3 oxidation state centre to adopt 4-, 5-, and 6-coordinate compounds resultant from dative bonding through dative ligands.

Table 1. Comparison of aluminum and copper properties ${ }^{(7)}$

\begin{tabular}{llll}
\hline & Aluminum & Copper & Units \\
\hline melting point & 660.32 & 1084.62 & ${ }^{\circ} \mathrm{C}$ \\
density, $\rho$ & 2.70 & 8.96 & $\mathrm{~g} \cdot \mathrm{cm}^{-3}$ \\
crystal structure & Face-centered cubic & Face-centered cubic & \\
space group & Fm3m & Fm3m & \\
thermal conductivity, $\lambda$ & 2.37 & 4.01 & $\mathrm{~W} \cdot \mathrm{cm}^{-1} \cdot \mathrm{K}^{-1}$ \\
mass thermal capacity, $C_{p}$ & 897 & 385 & $\mathrm{~J} \cdot \mathrm{kg}^{-1} \cdot \mathrm{K}^{-1}$ \\
electrical resistivity, $\rho$ & 27.1 & 17.1 & $\mathrm{n} \Omega \cdot \mathrm{m}$ \\
\hline
\end{tabular}

Unfortunately, aluminum is extremely oxophillic, producing one of the most stable oxides on the periodic table with an enthalpy of $\Delta G=21582 \mathrm{~kJ} / \mathrm{mol} .{ }^{(8)}$ As a result, pure metallic aluminum cannot be found in nature, but rather is available in hundreds of minerals which can be separated into five families, providing a breadth of material characteristics. ${ }^{(8)}$ Due to aluminum's oxophillic nature, the formation of $\mathrm{Al}_{2} \mathrm{O}_{3}$ is a very favourable process and any air leaks that may occur during film deposition with ultimately result in oxide formation. 
Furthermore, when dealing with aluminum metal organic compounds aluminum, synthesis and manipulation must be performed under inert atmosphere.

Once an aluminum metal deposition process has been established, there are other issues to be considered: the conducting aluminum interconnect must exhibit strong adhesion to the underlying surface. This may require the addition of a thin dielectric material which will enhance the adhesion. However it is achieved, diffusion of the aluminum into the dielectric material or a silicon substrate must be prevented. ${ }^{(9)}$ Since aluminum metal is oxophilic, diffusion of oxygen through other materials and into the aluminum layer is also of great concern, prompting the use of protective layers, such as metal nitrides, to the aluminum metal film. ${ }^{(9,10)}$

\subsection{Vapour Deposition}

The deposition of thin films through chemical vapour methods has been known for a long time. Over the years the processes have become increasingly more controlled and a number of variations on technique have emerged. As electronic devices rapidly decrease in size, the demand for high quality materials from film deposition techniques becomes more and more apparent. The available techniques have been extensively scrutinized and issues such as precursor volatility, deposition rate, step coverage, film quality, and film adhesion must all be considered when selecting a deposition method to suit the manufacturing of a device. ${ }^{(11,12)}$ When these processes are being scaled up for device manufacturing, it is also important to consider cost and availability of precursors and other materials, energy demands of the process, as well as toxicity and environmental impacts. ${ }^{(13)}$ 


\subsubsection{Chemical Vapour Deposition}

Chemical vapour deposition (CVD) is a set of deposition processes in which film deposition occurs via surface reactions at a substrate by vaporous precursors. Understanding the deposition thermodynamics and kinetics is important when trying to achieve high quality, reproducible films. As depicted in Figure 2, there are 8 processes which contribute to film growth. $^{(12)}$

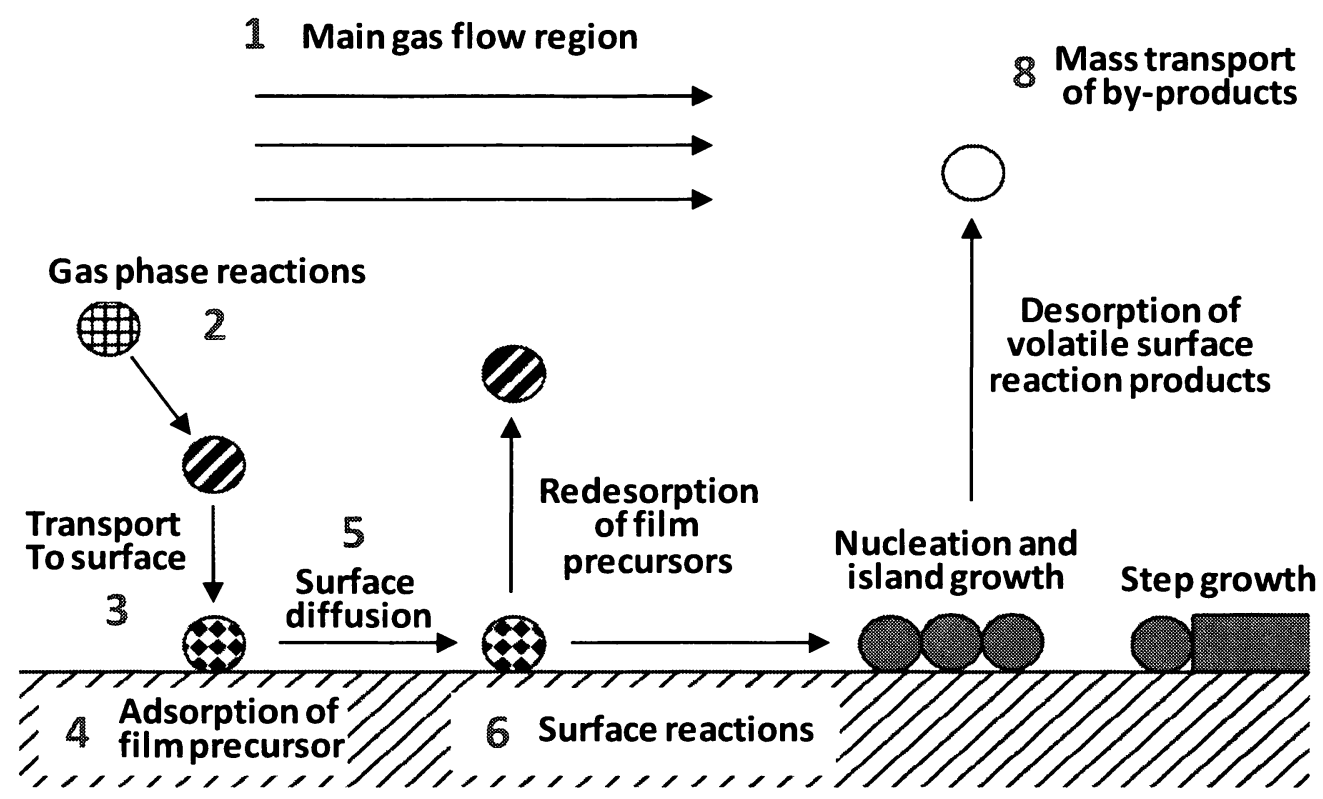

Figure 2. Scheme of general CVD processes ${ }^{(12)}$

In the main gas flow region (1), vaporous precursors are introduced, typically using an inert carrier gas. A differential in pressure across the deposition furnace helps to push the precursor vapour through the reactor, introducing the precursor to the substrate for deposition. Often in CVD processes, precursor compounds will undergo gas phase reactions (2) in order to produce surface reactive species. For a standard CVD process, multiple precursors can be introduced simultaneously to promote these gas phase reactions. This not however the case in atomic layer deposition (ALD) where the precursor introduced into the 
deposition chamber must be thermally robust and remain intact until reaching the surface, and multiple precursors must be introduced sequentially.

The transport of the precursor to the substrate surface (3), adsorption on the substrate (4), and surface reactions (6) define the film growth rate. Figure 3 demonstrates this temperature dependant relationship. At higher temperatures, the film growth rate is dictated by the transportation of precursor compound to the substrate. This is due to the increased rate of surface reactions. As adsorption and chemisorption occur nearly instantaneous at high temperatures, the growth of the film is limited instead by how fast the precursor molecules can make their way to the surface. In contrast, when the temperature of the reaction chamber is low, the rate of surface reactions diminishes, becoming detrimental to film growth.

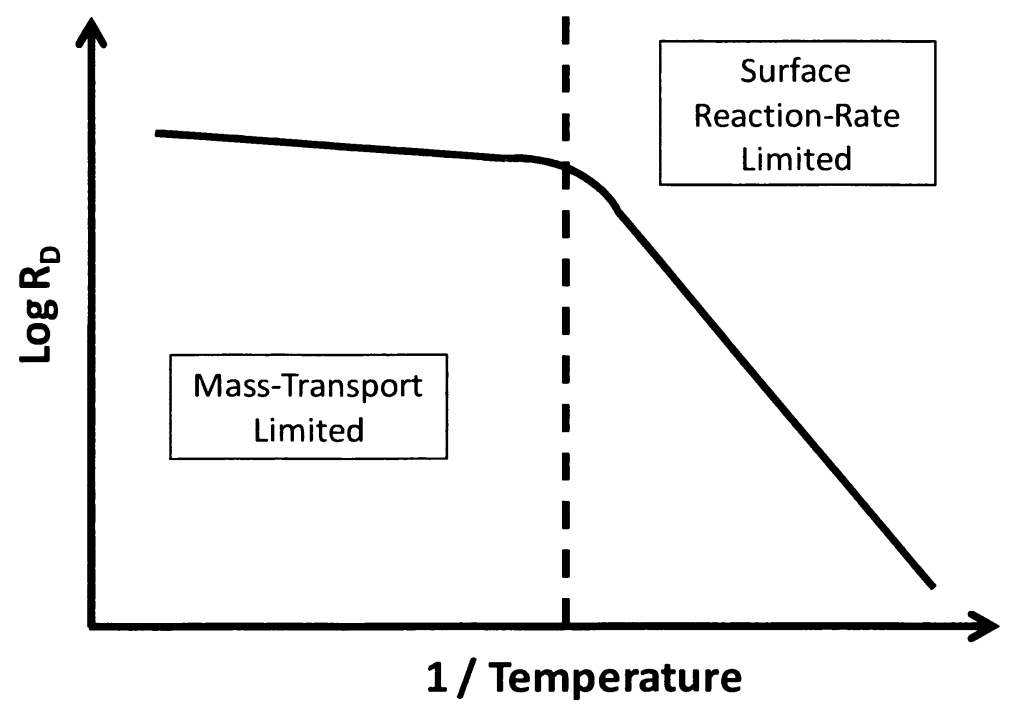

Figure 3. Limiting mechanism of overall deposition process ${ }^{(14)}$

In a CVD process, precursor compounds often adsorb onto the substrate surface and are able to undergo surface diffusion (5) prior to chemisorption. During this surface diffusion, the precursor can desorb which can greatly affect film growth in a mass-transport limited process. 
The ability for a precursor to remain adsorbed on to a surface is a probability known as the sticking coefficient which is both temperature and nucleation site density dependant. Surface diffusion often occurs across a surface as adsorbed species search out lower energy states. This often leads to island growth as adsorbed compounds often undergo surface nucleation next to other nucleated compounds, similar to crystal growth from seeding.

The final process in film deposition is desorption of by-products and their removal from the deposition chamber (8). By-products from the surface reactions should be volatile and nonhazardous as dictated when selecting/designing a precursor for vapour deposition. The removal of these by-products is essential to avoid contaminates from becoming trapped within the growing film. Since CVD is a continuous process, flow rate of the carrier gas must be sufficiently high. Where as in the case of ALD processes, the sequential introduction of precursors allows for introduction of a purge period within the process cycle to help remove by-products and unreacted precursor compounds after each layer of film has been deposited.

Since CVD can be controlled through a transport limited regime, the conformality and continuity of very thin films can be difficult to obtain. ${ }^{(9)}$ In order to gain conformal, continuous films at low thickness using CVD, the process must be performed at a low temperature, thus hindering the surface reactions and slowing the growth rate. This slow reaction rate during CVD can be detrimental to film quality as impurities from unreacted precursor compounds can become trapped from insufficient removal of by-products. ${ }^{(9)}$ Additionally, low temperatures often mean that the process is dependent on uptake of the precursor at the substrate, and the growth rate becomes very temperature dependant. This requires much greater technical 
control over the deposition. Thus, when depositing very thin films (on the order of 10nm), it is important to employ a highly controlled process such as atomic layer deposition (ALD).

\subsubsection{Atomic Layer Deposition}

Atomic layer deposition (ALD) was first introduced as Atomic Layer Epitaxy (ALE) in a patent by Suntola and Antson in $1977 .{ }^{(15)}$ ALE was named for its epitaxial growth, referring to an ordered monocrystalline film where the growing crystal face is defined by the substrate. In early ALE, substrates were placed on a rotating holder within a vacuum chamber. Rotation of the substrates over heated precursor vessels introduced sequential exposure, adding a level of control to film growth that was lacking in traditional CVD methods. ${ }^{(16)}$ Since the initial patent filed in 1977, ALE has been expanded to include growth of polycrystalline and amorphous films, focusing more on the controlled layer-by-layer aspect of the initial process and its ability to grow films with atomic precision, thus becoming denoted as atomic layer deposition (ALD). ${ }^{(17)}$

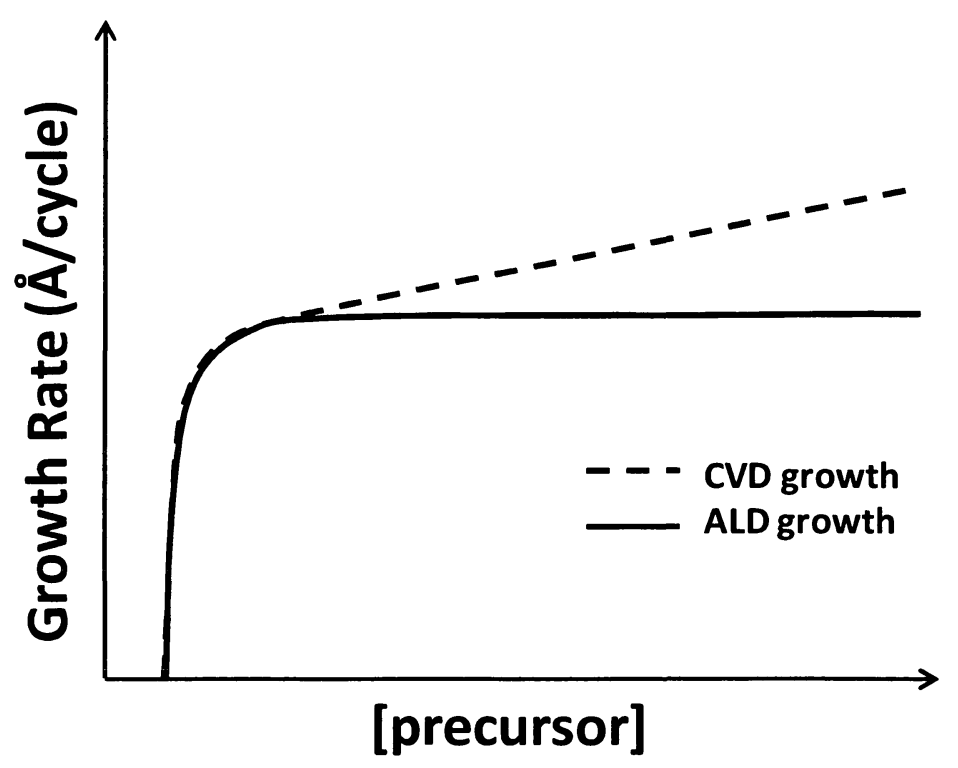

Figure 4. Growth rate: comparison of CVD and ALD 
ALD is a sub-class of CVD which possesses the advantage of self-limiting growth. Figure 4 presents the fundamental difference between ALD and any other CVD method where saturation of a surface occurs, preventing uncontrollable or continuous growth. The selflimiting behaviour that ALD exhibits can be achieved through precursor selection: components of the target film are supplied by vaporous compounds, selected to possess 5 characteristics. The most important of these characteristics is the Inability of the compound to react with like species within the reaction chamber. Once the vaporous precursor has reacted with the nucleation sites of a surface, no further reactions will occur, resulting in a saturated monolayer of growth. Since continual growth does not occur, a cyclic introduction of precursors is required to grow the film, adding another level of control to the technique.

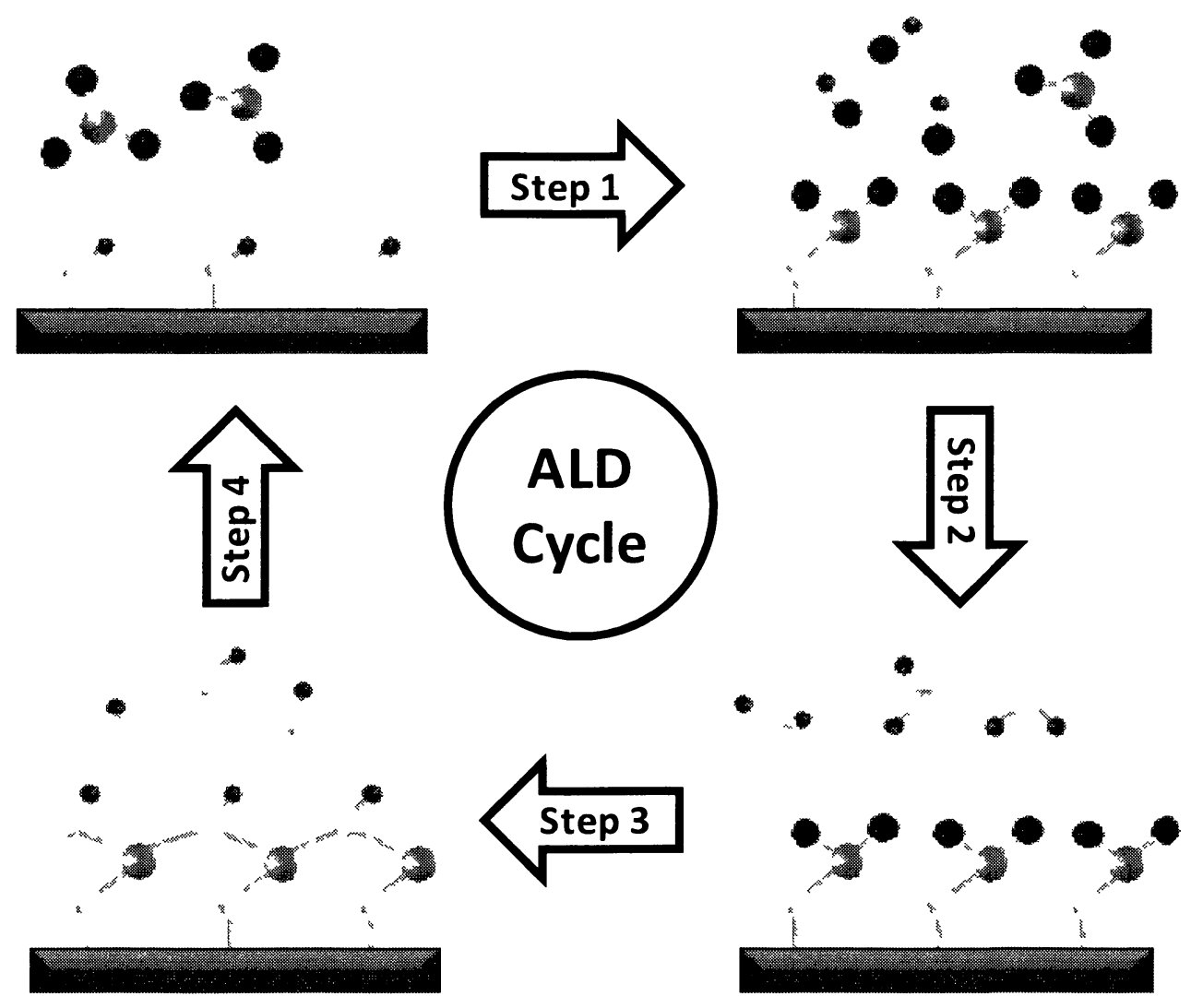

Figure 5. Schematic diagram of cyclic ALD process 
Through sequential introduction of film precursors, the target film can be precisely controlled and grown with atomic precision. The most extensively studied ALD mechanism is that of $\mathrm{Al}_{2} \mathrm{O}_{3}$, deposited using trimethylaluminum (TMA) and water precursors. ${ }^{(18)} \mathrm{A}$ simplified ball and stick diagram of this mechanism is depicted in Figure 5 demonstrating the 4 step growth cycle of a standard ALD process: ${ }^{(3 ; 18)}$

1. The metal precursor is introduced into the reaction chamber in the gas phase and chemisorbs to a preheated substrate at hydroxyl nucleation sites. In theory, all nucleation sites on the surface will undergo reaction with precursor compounds resulting in complete saturation of the substrate surface.

2. The surface reaction results in the release of a protonated ligand from the metal precursor. This by-product, along with any excess precursor (unable to find an available nucleation site) is purged from the reaction chamber using vacuum and/or a flow of inert gas.

3. Introduction of the secondary precursor typically provides the second component of the film. The secondary precursor reacts with the surface species to remove the protective ligands from the metal resulting in one complete layer of target film, also providing a fresh layer of nucleation sites on the surface.

4. The ligands that were released from the exchange reaction between the surface bound metal and the secondary precursor, along with any unreacted precursor, are removed from the reaction chamber using vacuum and/or a flow of inert gas. 


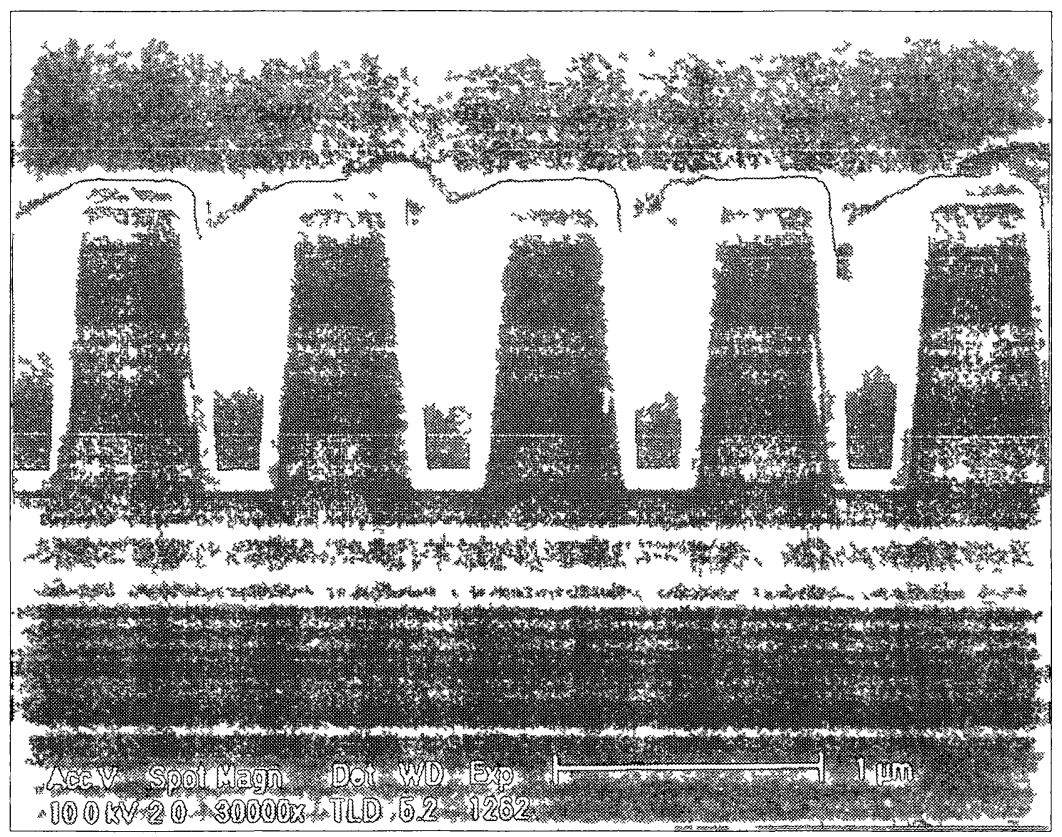

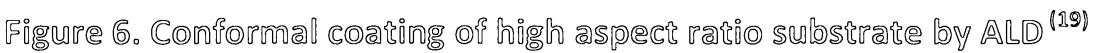

The benefors of the control ganed by depositnon usung ALD is evident un Fugure 6, where the folm growth is completely conformal such that the folm thuckness at the top of the patterned substrate is the same thuckness as the bottom of the wells Thus type of substrate is known as havong a high aspect ratro, defuned as the depth of the feature with respect to the width ${ }^{(20)}$ The duffucultues that arse when artemptng conformal folm depositnons on hogh aspect ratuo substrates is mandy a knnetuc argument where folm growth is dependent on the diffusion of the precursor nnto the feature, allowng for surface reactons to occur at all nucleation sites on the surface ${ }^{(20)}$ Thus demand for controlled folm deposition hughlights the benefut of usung ALD over other CVD methods

Conventronal ALD, as descrubed thus far, is known as thermal ALD sunce the actovation energy which druves the surface reactions is provided by heatng Another form of folm deposition which demonstrates selfalomoted growth is known as plasma enhanced ALD, or 
PEALD. This process does have a known procedure for growing aluminum metal films. PEALD gains its activation energy from a plasma source, allowing for the substrate to be held at a much lower temperature than in thermal ALD. ${ }^{(9)}$ For PEALD, the metal precursor is introduced in the reaction chamber and allowed to react to the substrate surface as in thermal ALD. After purging the chamber, the secondary, non-metal containing precursor is activated by $r f$, microwave, or electron cyclotron resonance to generate a plasma. ${ }^{(9 ; 3)}$

In the case of aluminum metal PEALD, hydrogen plasma is generated to reduce the surface bound aluminum species to yield a layer of aluminum metal. This method can produce films of higher purity, higher density and at lower temperatures then thermal ALD, however these benefits are often outweighed by the disadvantages. One potential draw back in PEALD is the deactivation of reactive species due to wall collisions when attempting depositions on substrates with high aspect ratios. ${ }^{(9)}$ Also, due to the harsh conditions during PEALD processes, the exposure of plasma can be detrimental to device components (such as gate oxides) or detrimental to the film itself. ${ }^{(9)}$ Thus, PEALD is not suitable when depositing films on surfaces composed of various components, as is often the case when depositing interconnect materials for electronic components. ${ }^{(9)}$

Aluminum metal deposition by thermal ALD is yet unknown, making the research reported within this thesis to potentially have a large impact on interconnect device research and development. 


\subsection{Precursor Design}

Early in vapour deposition process development, not much consideration was given to the precursors used for the process. Typically, metal compounds were selected from a chemical catalogue based on their characteristic vapour pressure and molecular weight. A high level of success came from establishing processes in this manner, mostly using homoleptic metal compounds: halides, alkanes, and alkoxides. ${ }^{(21)}$ As demands on film quality, thickness, and composition became more rigorous, deposition techniques began to become altered in order to produce desired results.

This is precisely why techniques such as plasma enhanced CVD and ALD exist. Using the classic ALD process of TMA and water to produce $\mathrm{Al}_{2} \mathrm{O}_{3}$ films as an example, it is apparent that this process established TMA as the precursor for aluminum. Thus, when trying to produce a method for depositing aluminum metal films, instead of investigating other aluminum precursors, plasma enhanced ALD was employed. Aluminum metal thin films were produced with high precision, but with a much higher energy consumption then necessary.

The design of precursor compounds is a powerful tool to alleviate excess use of energy in a deposition process. Depending on the deposition technique, the characteristics required of a precursor can vary. The relationship between the metal precursor (or primary precursor) and the secondary precursor is also of great importance. Through careful design, precursors can be tailored for a process adding control to the deposition mechanism. 


\subsubsection{Metal Precursors}

When designing precursors for atomic layer deposition, there are 5 characteristics which are desirable:

1. High Volatility: The precursor must be introduced into the deposition chamber as a gaseous vapour. It is advantageous for a precursor to volatilize cleanly, and at low temperature; this can be achieved by designing the precursor to have a low molecular weight. Also, by coordinatively saturating the metal centre, the potential for oligomerization is removed.

2. Low Melting Point: During film deposition, it is important for the amount of precursor introduced in each pulse to be consistent. To achieve this, the precursor would ideally be in a liquid state, thus providing a constant vapour pressure. This characteristic can be achieved through branching and asymmetry through ligand design or using heteroleptic ligand systems.

3. Thermally Stable: When designing a precursor, it is important that the precursor remains intact and stable until it reached the substrate surface and chemisorbs. This can be achieved by selecting ligand systems which have strong bonds to the metal centre which can withstand deposition temperatures in reduced pressure environments.

4. Chemically Reactive: Atomic layer deposition requires the vapour precursor to undergo chemisorption onto the substrate surface. Even though strong bonds to the metal centre are desirable to gain thermal stability, the bond must be less energetically 
favourable then chemisorption to the substrate surface. Thus, the metal to ligand bond strength must satisfy this balance.

5. Self-Limiting Behaviour: The entire basis of atomic layer deposition is that film growth is completely uniform. Unlike CVD processes where film growth is continuous, ALD requires saturation of a surface such that excess precursor will not react. This characteristic can be attained by using ligands which provide steric protection for the metal centre, guarding against further reaction with gas phase components until the secondary precursor is introduced.

The characteristics listed above are also desirable for CVD precursors, with the exception of self-limiting behaviour. Since CVD is a continuous process, a precursor which incorporates steric protective ligands would only hinder the process, resulting in slowed deposition rates.

\subsubsection{Secondary Precursors}

Secondary precursors have traditionally not been subjected to the same level of design as the primary precursor; its role is typically to reduce or oxidize the metal centre and sometimes to provide a second component to the film. In CVD processes, unless using a single source precursor, a secondary precursor will often be introduced simultaneously with the primary precursor to promote gas phase reactions.

In ALD processes, a secondary precursor is always required, whether it is to provide a second component to the film, or to simply reduce or oxidize the metal at the surface. Often, water or ammonia will be introduced to provide a source of oxygen or nitrogen when growing metal oxide or metal nitride films, respectively. ALD processes to form metal films are slightly 
more complex than metal oxide or metal nitride film processes and have been found to grow from 3 different simplified mechanisms: ${ }^{(9)}$

1. Metal precursor + reducing agent $\rightarrow$ metal film

Example: $\mathrm{TaCl}_{5}+\mathrm{H} \rightarrow \mathrm{Ta}^{0}$

2. Metal precursor + oxidant $\rightarrow$ metal film

Example: $\mathrm{Ru}(\mathrm{Cp})_{2}+\mathrm{O}_{2} \rightarrow \mathrm{Ru}^{0}$

3. Metal precursor + oxidant $\rightarrow$ reducing agent $\rightarrow$ metal film

Example: $\mathrm{Ni}(\text { acac })_{2}+\mathrm{H}_{2} \mathrm{O} \rightarrow \mathrm{NiO}+\mathrm{H}_{2} \rightarrow \mathrm{Ni}^{0}$

The addition of a reducing agent as in method (1), is the most obvious process. Unfortunately, the use of a forming gas reducing agent $\left(5 \% \mathrm{H}_{2}\right.$ in $\left.\mathrm{N}_{2}\right)$ is often not strong enough to reduce the metal surface species. As in the example, when using halide precursors, the strength of the halide-metal bond is much too strong to be reduced by forming gas. Instead, the introduction of hydrogen plasma is used to reduce the surface species. The use of hydrogen plasma is a harsh process as discussed above. This excessive use of energy is an excellent example of how metal precursor design can be used to diminish process costs by allowing for the use of simpler secondary precursors such as forming gas.

The addition of an oxidant as a secondary precursor when attempting the deposition of a metal film is counter intuitive. Never the less, methods (2) and (3) did just that. The two methods were found by accident as metal oxide depositions being attempted serendipitously resulted in metal films. ${ }^{(9)}$ Method (2) was found to occur for both Ru and Pt deposition: as oxygen was brought into the deposition chamber, it was held there static for a period in order to promote adsorption at the surface to promote oxidation of the surface bound metal. 
However, the $\mathrm{O}_{2}$ instead oxidized part of the hydrocarbon ligand to form carbon oxides and water resulting in reduction of the metal. ${ }^{(3,22)}$

The third method of metal deposition known in the literature is that shown above as method (3). A known deposition method for nickel oxide is first performed using water vapour as an oxidant. The film is then reduced post-deposition using forming gas. ${ }^{(23)}$ This method demonstrates a way around using hydrogen plasma using a very simple secondary precursor of water vapour. Unfortunately, this method is not found to work for many metals as the deposited metal oxide must be susceptible to reduction. In the same body of work, $\mathrm{Cu}$ and $\mathrm{Pt}$ were unable to be reduced by the same method as their oxides are much stronger than that of NiO. ${ }^{(23)}$ To overcome this barrier, forming gas was introduced sequentially to reduce the film after each deposition cycle. This method proved to be more successful as $\mathrm{Cu}$ and Pt metallic films were achieved. ${ }^{(23)}$

The method by which a metal film is deposited is completely dependent on the target material; in no way are the 3 mechanisms described above able to be applied to just any process. What they do demonstrate is that the role of the secondary precursor is essential. The synergistic effects of the metal precursor and secondary precursor in ALD processes cannot be ignored. It is clear by these few examples that ALD processes remain fairly unknown as a diverse set of approaches exist resulting in known metal processes for only 8 materials (Ta, Ti, $\mathrm{W}, \mathrm{Cu}, \mathrm{Ni}, \mathrm{Pt}, \mathrm{Ru}$, and $\mathrm{Al}){ }^{(9)}$ Of these 8 materials, only 5 have known thermal ALD processes. ${ }^{(9)}$ From the methods above, it is clear that the used of PEALD is not necessary for the production of metallic films, and through precursor design, more efficient processes can be gained. 


\subsubsection{Amidinates and Guanidinates}

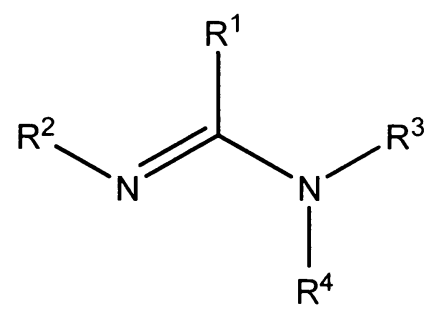

Figure 7. General structure of amidine compounds

Amidines represent a large class of compounds encompassing any compound which exhibits the general structure shown in Figure 7. The ' $R$ ' groups shown can be substituted as hydrogen, alkyl, or amide, and can even be substituted to form heterocyclic structures. ${ }^{(24)}$ When $\mathrm{R}^{4}$ is removed and the amidine is bound to a metal centre, metallocycle amidinate compounds are formed. In this body of work, the amidinates are symmetrically trisubstituted, such that $R^{2}$ and $R^{3}$ are equivalent and $R^{1}$ represents either an alkyl or amide group. The nomenclature of amidines is based around the $R^{1}$ group such that compounds where $R 1$ is a methyl group, the structure is known as an acetamidinate, and when $R^{1}$ is substituted for an amide group, the structure is classified as a guanidinate. ${ }^{(24)}$

Amidinate and guanidinate ligand systems are of particular interest in precursor design as they contribute many of the desirable characteristics required for an ALD precursor. They provide excellent steric protection of the metal centre allowing for self-limiting behaviour. Their coordination to the metal centre occurs through a delocalized bond, shown in Figure 8, which provides both thermal stability as well as aiding in the coordinative saturation of the metal centre preventing oligomerization (which would increase the molecular weight and decrease the volatility of the compound). 
A)

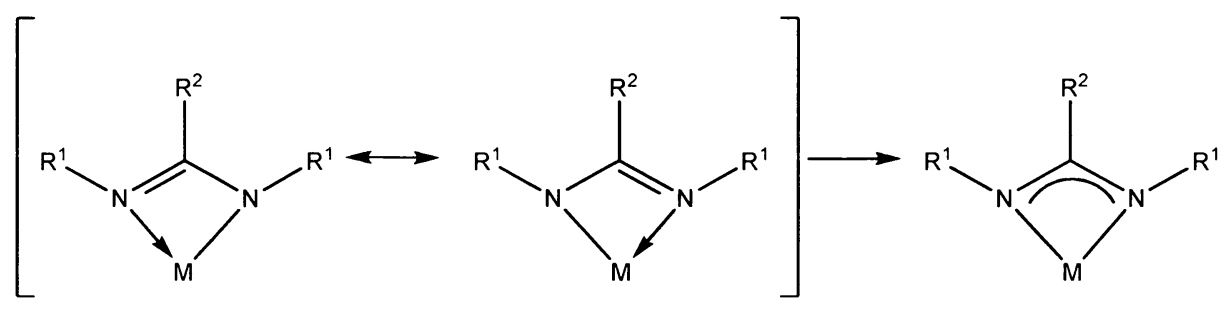

B)

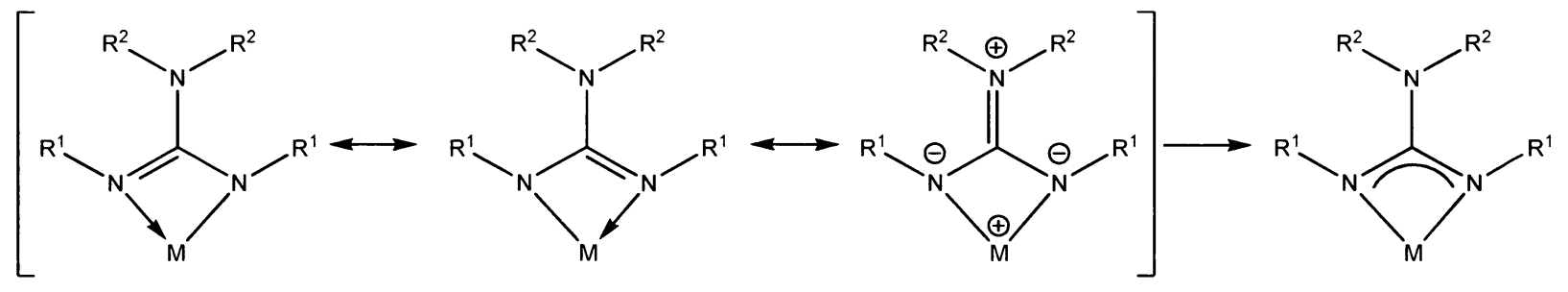

Figure 8. Resonance structures of A) amidinate ligands, and B) guanidinate ligands

Amidinates covalently bind to a metal centre through one of the nitrogen atoms, as shown in Figure 8 . The nitrogen which participates in $\pi$ bonding with the quaternary carbon possesses a lone pair which can contribute donation to the metal centre through a dative bond. Since either nitrogen can possess the double bond, a resonance hybrid structure is often shown depicting each nitrogen as having equal contribution to binding with the metal centre. For guanidinate ligands, a third resonance structure exists in which the double bond can be position between the quaternary carbon and the exocyclic nitrogen as shown in Figure 8B).

As discussed above, aluminum is a hard acid and easily accepts electron density. As such, for an aluminum metal centre in a +3 oxidation state, one, two or three amidinate ligands can be coordinated to produce 4-, 5- and 6-coordinate precursors known as mono, bis and tris amidinates, respectively. These structures are shown in Figure 9: 

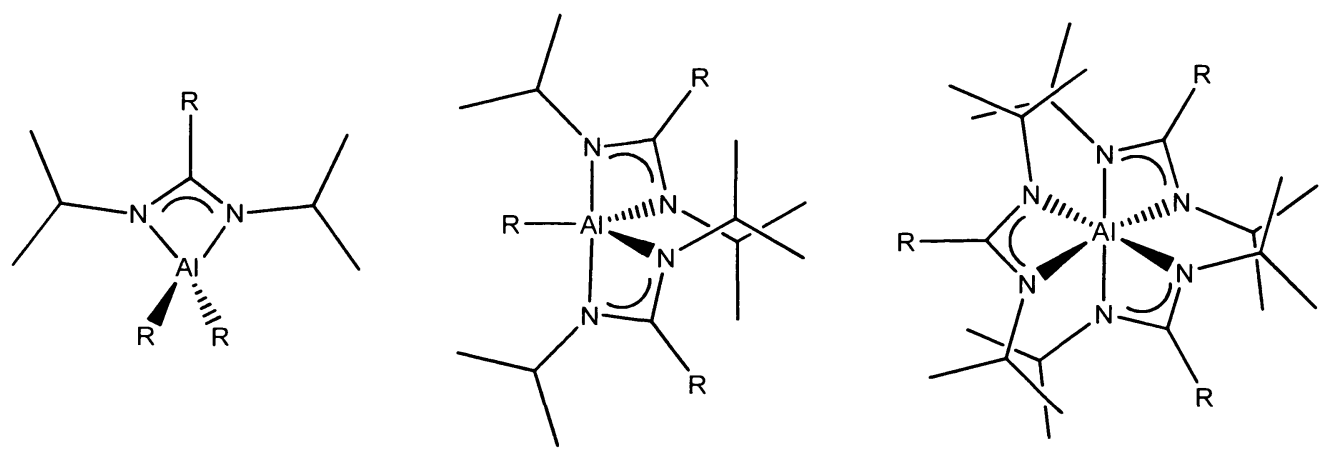

Figure 9. Amidinate and guanidinate coordination to aluminum (iii) metal centre

Amidinates offer excellent properties as ALD precursors: they help coordinatively saturate the metal centre, provide strong bonding to the metal centre, offer tunable thermal characteristics, and possess branching and steric protection. Amidinates are very suitable for use in precursor design, however, some thermal instability exists as amidinates have been found to suffer from carbodiimide deinsertion $(25,26,27,28)$, and more recently beta hydride abstraction ${ }^{(27,29)}$. Despite this small amount of thermal instability, the tunable nature of this class of compounds remains advantageous.

\subsection{Surface Chemistry of Metal Films}

\subsubsection{Surface Nucleation Sites}

The surface reactions that occur during vapour deposition methods dictate the growth rate, density, and morphology of the resultant film. In order to form continuous, pin-hole free thin films the sites available for nucleation are extremely important. ${ }^{(18)}$ Thus, understanding the surface species and the density of the nucleation sites throughout the deposition process is advantageous when trying to produce high quality films.

Traditionally films are grown on substrate surfaces on which the density of nucleation sites can be controlled through pre-treatment. ${ }^{(30,31)}$ For example, the density of hydroxyl sites 
on a silica surface can be controlled by heating the substrate in ambient atmosphere: the higher the temperature, the lower the density of hydroxyl sites on the surface. ${ }^{(32)}$ This trend can be seen in Figure 10, where a $\mathrm{Si}^{29}$ NMR study was used in conjunction with weight loss monitoring to track the dehydration of the silica surface. Figure 10B) presents a representation of the surface species that may exist on the surface of the silica as dehydration occurs with elevated temperature. ${ }^{(30 ; 31)}$

A)

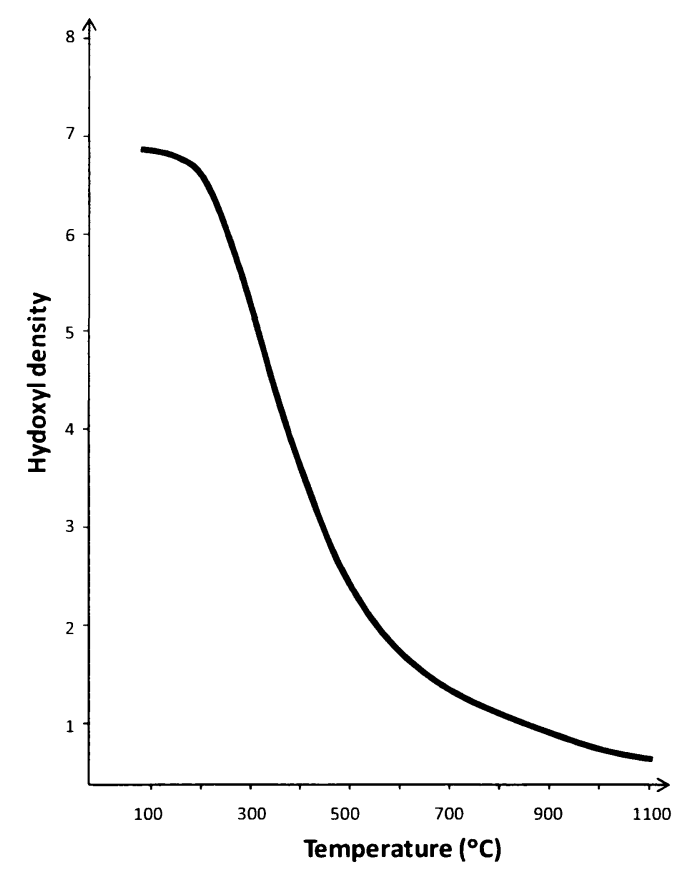

B)

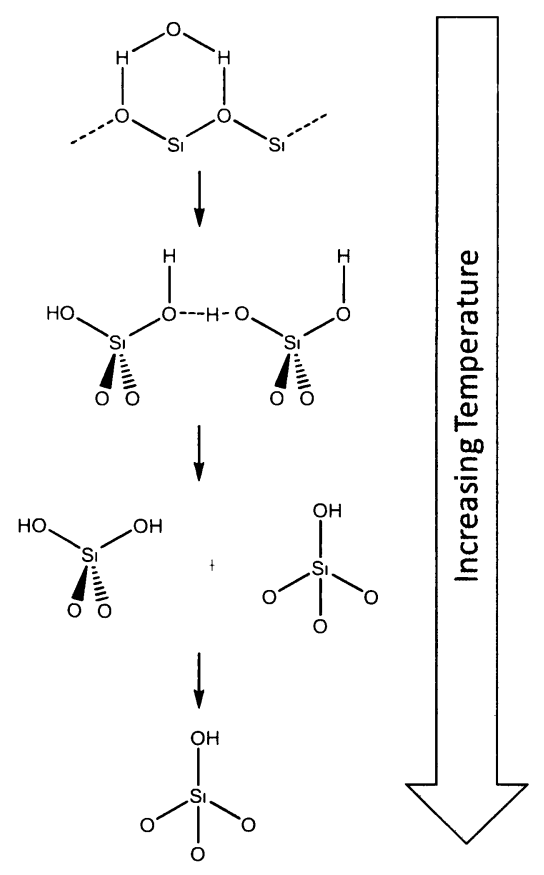

Figure 10. Hydroxyl density on high surface area silica A) general trend based on $\mathrm{Si}^{29} \mathrm{NMR}$ and weight loss studies ${ }^{(32)}$ with B) proposed surface species ${ }^{(30 ; 31)}$

Although the control of hydroxyl sites on an oxide surface is well known, the manner in which precursor compounds nucleate on the surface of metals is still unclear. Thermal ALD processes do exist for the deposition of $\mathrm{W}, \mathrm{Cu}, \mathrm{Ru}$, and Pt metal films, thus the reaction of a precursor with a metal surface must have the potential to proceed in a controlled manner. ${ }^{(9 ; 18)}$ The question becomes: how does the precursor nucleate on the metal surface without 
precursor decomposition? Upon deposition of $\mathrm{Ni}$ metal films this nucleation problem can be avoided by first depositing a $\mathrm{NiO}$ film, through the common use of an oxidant as a secondary precursor, followed by a reduction using $\mathrm{H}_{2}$, post deposition. ${ }^{(33)}$ Unfortunately, when depositing films of oxophillic metals, such as aluminum, the energy barrier required to be overcome and release the oxygen is much too great.

Typically, gas phase precursors undergo exchange reactions at the substrate surface, eliminating one of the precursor's ligands through protonation by the nucleation site. Another factor in the surface chemistry of the deposition process is the ligand which will be removed from the precursor upon exchange reaction at the surface. ${ }^{(34)}$ This exchange reaction can be controlled through the design of the precursor; by using mixed ligand, or heteroleptic, precursor compounds, one can help control which ligand will be released in the surface reaction. Typically, heteroleptic precursors for ALD are designed to have a protective ligand which is not required to undergo surface reaction, rather it must be susceptible to reaction with the secondary precursor in the process. The other ligand in the compound must react with nucleation sites on the surface. This surface reaction can be controlled through the bond energy between the metal and ligand. As discussed previously, the bonds formed at the surface must be more favourable than the bonds broken in the precursor. This aspect of the deposition process provides added control and tunability.

Although the precursor design can be optimized to promote reactions at the substrate surface, the presence of nucleation sites on the surface remains crucial. Ongoing studies into how nucleation occurs during metal deposition processes are essential for understanding the mechanism of deposition. 


\subsubsection{Metal Hydrides}

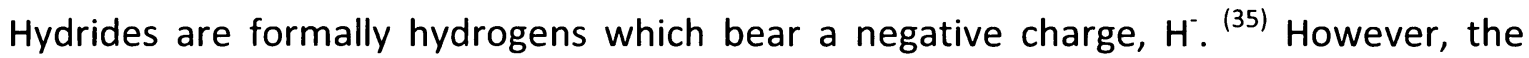
term hydride is also used, and accepted, as a metal bound hydrogen, or hydrido ligand. ${ }^{(36,37)}$ Often, metal bound hydrogens behave as anions, participating in bonding which exhibits ionic behaviour. The representation of $\mathrm{M}-\mathrm{H}$ bonding can be seen in Figure 11 . This ionic behaviour stems from the valence orbital energy of the hydrogen in comparison with that of the metal centre. Depending on the metal centre, and the ligand systems that surround them, the polarity of the $\mathrm{M}-\mathrm{H}$ bond will vary. ${ }^{(36)}$

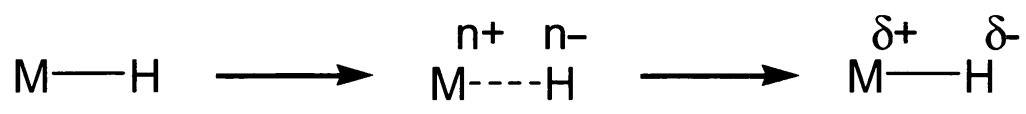

Figure 11. Representation of $\mathrm{M}-\mathrm{H}$ bond

The thermodynamic stability of an $\mathrm{M}-\mathrm{H}$ bond is in competition with the formation of $\mathrm{H}$ $\mathrm{H}$ and $\mathrm{M}-\mathrm{O}$ (in the presence of an $\mathrm{O}$ source). ${ }^{\left({ }^{36)}\right.}$ As discussed above, aluminum, in particular, has a strong affinity for oxygen. Thus, in the presence of air, an aluminum hydride, as well as most other metal hydrides, will decompose almost immediately at room temperature. As such, it is essential for hydride synthesis and compound manipulation to be performed under inert atmosphere.

Often, metal hydrides are investigated as catalysts or reaction intermediates in hydroformylation, olefin isomerisation and hydrogen exchange reactions. ${ }^{(37)}$ Some research into the use of metal hydrides for deposition purposes has been previously reported. The use of amine adducts of $\mathrm{AlH}_{3}$ have been used to deposit $\mathrm{AlAs}$ and $\mathrm{Al}_{\mathrm{x}} \mathrm{Ga}_{1-\mathrm{x}} \mathrm{As}$ films by metalorganic chemical vapour deposition (MOCVD). ${ }^{(38)}$ Amidinate aluminum, gallium, and indium hydride 
compounds have also been investigated previously as precursors for CVD. ${ }^{(39,40)}$ However, the amidinates used in these cases consisted of large bulky, aromatic substituted amidinate ligand systems leading to heavy compounds which were difficult to volatilize. ${ }^{(40)}$

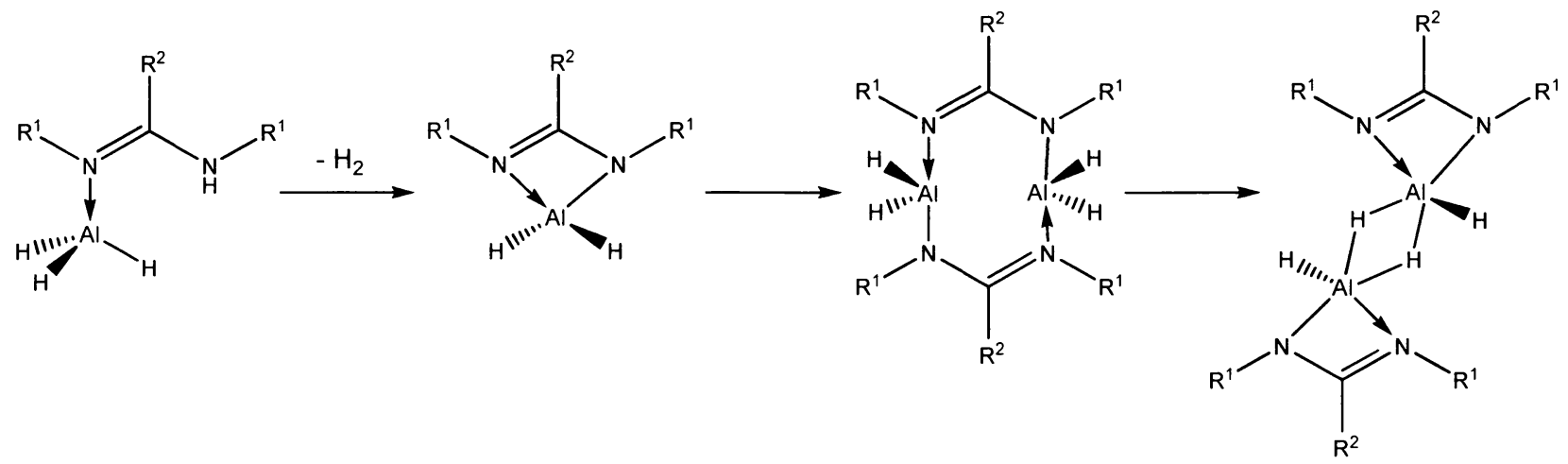

Figure 12. Dimerization in amidinate aluminum hydrides ${ }^{(40,39)}$

Since the ligand systems used for the amidinate metal hydrides were aromatic and bulky, an attempt at lowering the molecular weight by producing a 4-coordinate monoamidinate metal dihydride was attempted. Figure 12 shows the hydrogen elimination synthesis to the 4-coordinate compound as well as the subsequent dimer formations (amidinate bridged and hydride bridged in the case of bulkier R groups). This dimer formation occurs as the aluminum centre does not receive enough stabilization or protection from the two hydrido ligands leading to the formation dimeric compounds to alleviate the potential energy of the compound. From this dimeric compound formation, it is evident that the production of dihydrido aluminum compound are much too unstable, corroborating results collected during an honours research project which preceded this work. ${ }^{(41)}$

As evident by this dimeric bridging, the oligomerization of these compounds is also of concern. Instead of amidinate and hydrogen bridging between two metal centres, it is possible 
for bridging to form extended chains of metal centres. ${ }^{(36 ; 37 ; 39 ; 40)}$ In contrast to the 4 -coordinate compound attempts, the production of 5-coorinate bisamidinate monohydrido aluminum compounds appears more promising, resulting in monomeric compounds. The chelation of the two amidinate ligands helps coordinatively saturate the aluminum centre helping to stabilize the hydride, preventing oligomerization. ${ }^{(40)}$

\subsection{Project Overview}

The objective of producing an aluminum containing precursor for the purpose of depositing aluminum metal films by ALD, began as a Bachelor of Science honours project, preceding this current body of work, in $2007 / 2008 .{ }^{(41)}$ The use of a known aluminum precursor for aluminum oxide $A L D ;\left[\mathrm{MeC}\left(\mathrm{N}^{\mathrm{P} P r}\right)_{2}\right] \mathrm{AlEt}_{2}{ }^{(25)}$, was found to be unsuccessful as a precursor for aluminum metal deposition. ${ }^{(41)}$ The ethyl groups were believed to possess too strong of an interaction with the metal center, resulting in a failure to chemisorb onto the film surface void of hydroxyl nucleation sites. ${ }^{(41)}$ This theory initiated the use of aluminum hydrides as it was thought that the strength of the aluminum-hydrogen bond might be stable enough to withstand thermolysis, but would also be weak enough to promote reaction at the substrate surface. ${ }^{(30)}$ Initial synthesis of hydride compounds explored the use of formamidinate ligands, ultimately leading to the first aluminum metal deposition. ${ }^{(41)}$

The move away from formamidinate ligands for this body of work was an easy decision to make. Formamidinate aluminum hydride compounds were hard to purify; their honey-like consistency made the removal of impurities by distillation, sublimation, or even recrystallization rather difficult. The synthesis proved to be extremely difficult. Furthermore, a study into the relative thermal stability between aluminum formamidinate, aluminum 
dimethylguanidinate, and aluminum acetamidinate, presented a trend where the strength of the bond between the quaternary carbon in the metallocycle to the exocyclic moiety dictated the ligand stability. ${ }^{(41,26)}$ The formamidinate was found to deinsert diisopropyl carbodiimide ('Pr-CDI) at a relatively low temperature compared to the guanidinate, where as the acetamidinate was found to be completely stable throughout the study.

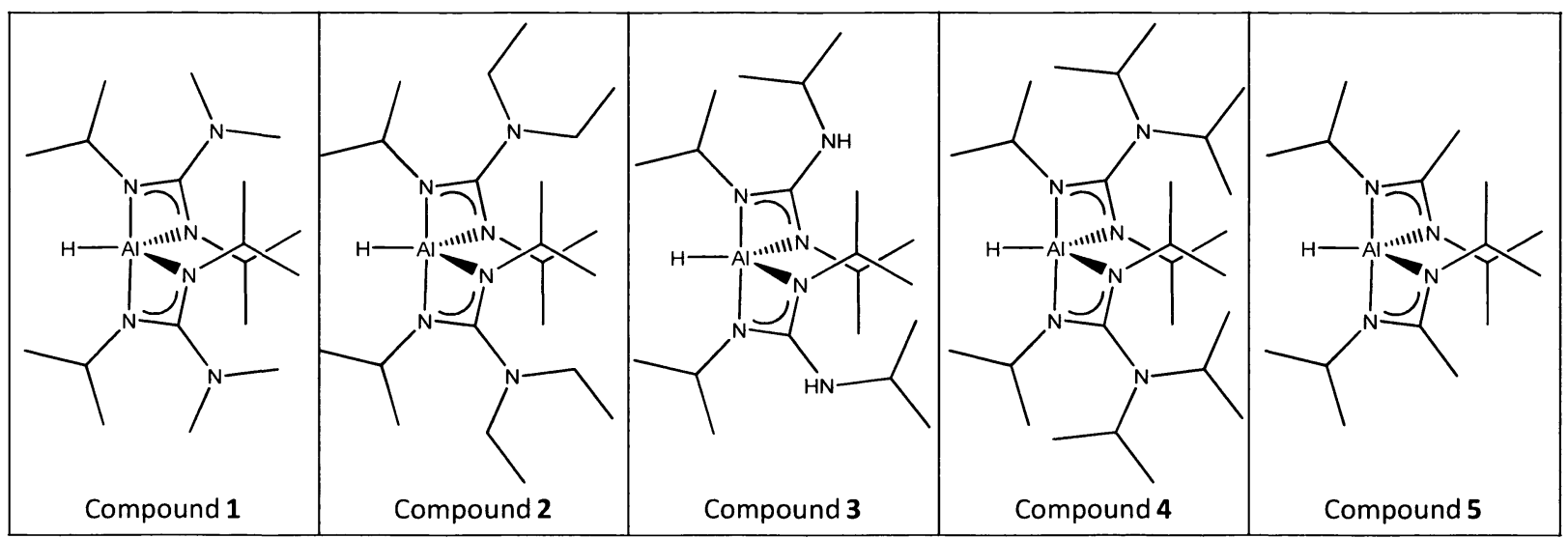

Figure 13. Structural representation of compounds 1-5

Based on the promising results from previous research, ${ }^{(41)}$ an exploration into the use of guanidinate and amidinate aluminum hydrides began in hopes of developing a suitable precursor for aluminum metal deposition. Encompassed in this thesis is an account of the synthesis of compounds 1-5, depicted in Figure 13, using hydrogen elimination and ligand exchange reactions. Air sensitivity of the compounds will be discussed as well as their structural determination through single crystal X-ray diffraction ( $s c X R D$ ), and thermal characterization using thermogravimetric analysis (TGA) and nuclear magnetic resonance (NMR) studies. Pulsed chemical vapour deposition (CVD) experiments are subsequently described and resultant films characterized using powder X-ray diffraction (pXRD), and scanning electron microscopy (SEM). Difficulties in film characterization using other techniques will be discussed. 


\section{HETEROLEPTIC ALUMINUM COMPOUNDS}

\subsection{Synthesis}

When designing precursors for deposition processes, it is essential for the synthetic method to be facile and economical such that scale up of precursor production may be possible. The synthesis of amidinate compounds has been thoroughly studied and methods such as salt metathesis, alkyl elimination, and ligand exchange are all fairly straight forward, often allowing for one-pot syntheses. ${ }^{(24,42,43,44,45)}$ Amidinate aluminum hydrides have been previously reported for amidinates containing aromatic groups, however compound synthesis was performed at $-78^{\circ} \mathrm{C} .{ }^{(39,40)}$ Synthesis of compound 1 was previously reported by our group in 2005 , but has since been tuned for more reproducible results. ${ }^{(46)}$ Herein, the synthesis of compounds 1-5 through facile hydrogen elimination and ligand exchange methods at room temperature are described.

\subsubsection{Hydrogen Elimination}

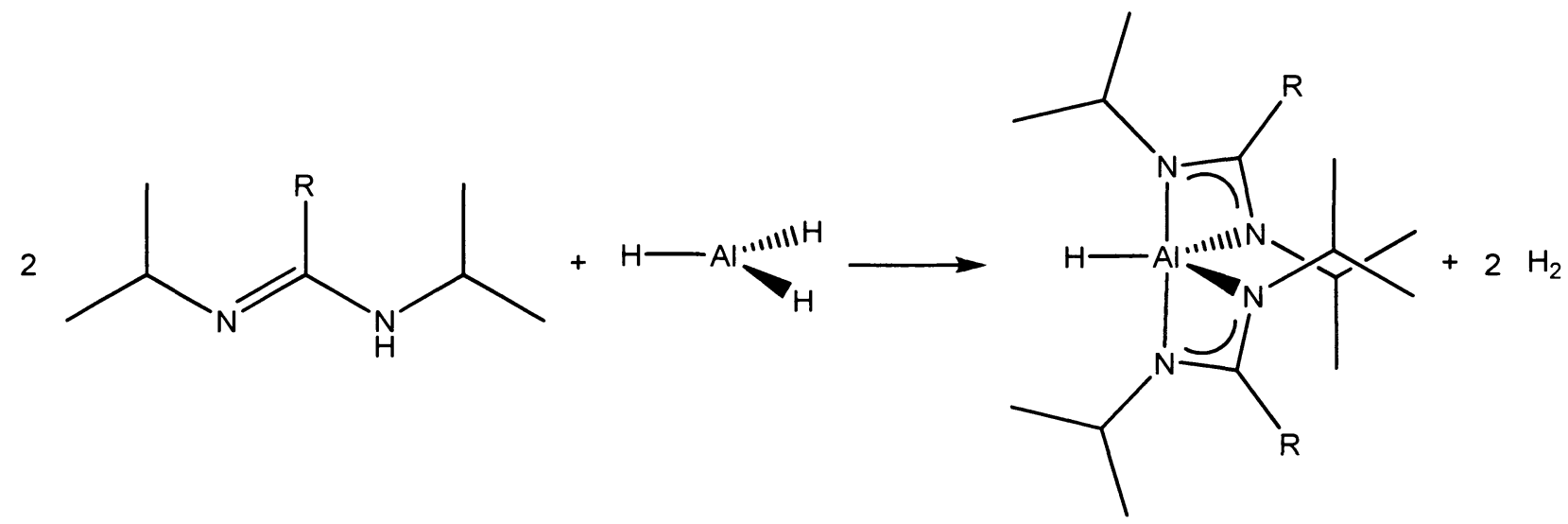

Figure 14. Hydrogen elimination

Compounds 2, 3, and 4 were synthesized through a similar method in which a guanidine is added to $\mathrm{AlH}_{3}$ in proper stiochiometric ratio, eliminating hydrogen gas to produce the desired 
bisguanidinate aluminum monohydride, as shown in Figure 14. Before this step can occur, the guanidine ligands must be synthesized. In order to produce a guanidine with a disubstituted amide, in which is to become the exocyclic position, a 4-step process is required:

1. Alkyl elimination between $\mathrm{Li}-\mathrm{Me}$ or $\mathrm{Li}-{ }^{\mathrm{t}} \mathrm{Bu}$ and the secondary amine to produce $\mathrm{Li}-\mathrm{NR}_{2}$

2. Insertion of 'Pr-CDI across the $\mathrm{Li}-\mathrm{N}$ bond to form the lithium guanidinate

3. Addition of one equivalent of water to hydrolyse the ligand

4. filtration to remove $\mathrm{LiOH}$, drying with $\mathrm{Na}_{2} \mathrm{SO}_{4}$ and filtration to isolate the desired guanidine

For preparation of the guanidine possessing a primary amide in the exocyclic position (as in the case for ligand preparation for compound 3), a simple insertion of 'Pr-CDI across the $\mathrm{N}-\mathrm{H}$ bond can occur. This process is preformed neat with heating to $115^{\circ} \mathrm{C}$ for 18 hours.

Compounds 2, 3, and $\mathbf{4}$ were synthesized upon slow addition of a solution of guanidine in toluene to a solution of $\mathrm{AlH}_{3}$ in toluene. The elimination of $\mathrm{H}_{2}$ was found to be an exothermic process, as one would expect. Thus, the $\mathrm{AlH}_{3}$ solution was cooled in a $-30^{\circ} \mathrm{C}$ freezer for $5-10$ minutes prior to addition, and the guanidine solution was often added using a dropping funnel to control the reaction rate. If the reaction was performed too quickly, enough thermal energy would be released causing the reaction flask to heat up and cause decomposition. A dropping rate of roughly 15 drops per minute was employed either by dropping funnel of by hand using a pipette. Once addition was complete, reactions were left stirring for a number of hours to ensure complete reaction. Compounds 2,3 , and 4 were isolated by crystallization at $-30^{\circ} \mathrm{C}$ after concentration by reduced pressure. Isolated compounds were usually pure by ${ }^{1} \mathrm{H} N \mathrm{NR}$ and did not require further purification methods. 


\subsubsection{Ligand Exchange}

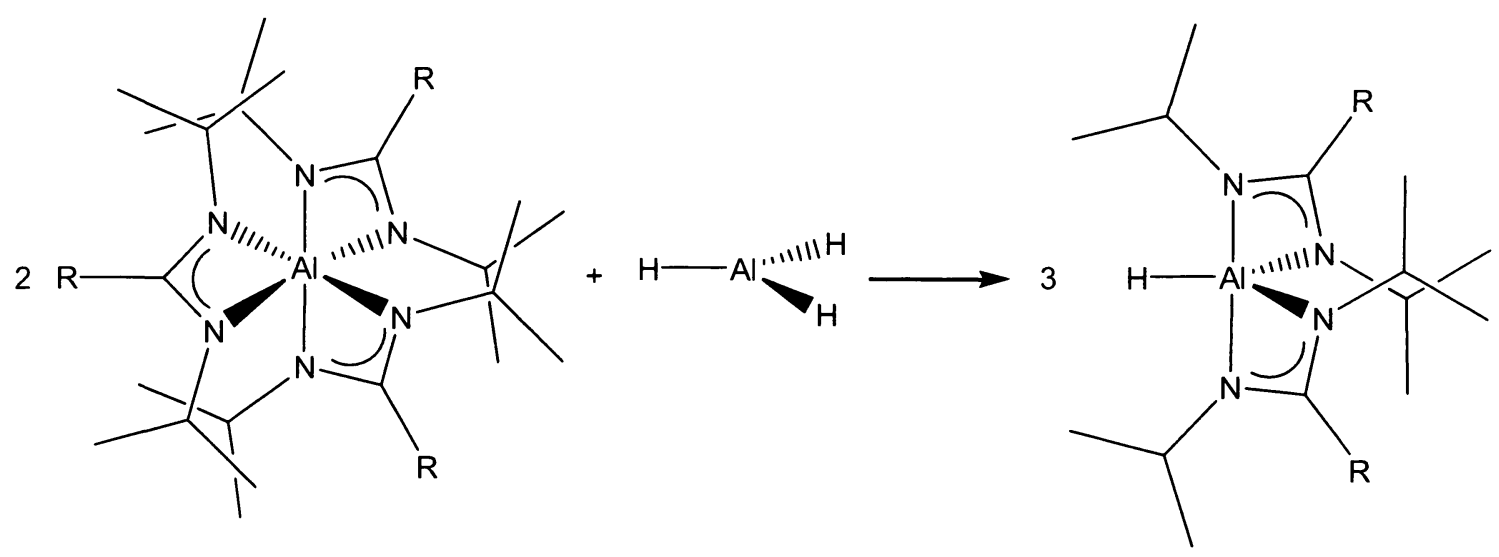

Figure 15. Ligand exchange

Ligand exchange reactions were first described by our group in $2005,{ }^{(47)}$ and have since been extensively investigated. $(25,46,48)$ The reaction method is a facile route to heteroleptic metal compounds where stoichiometic addition of homoleptic metal compounds is employed to tailor the ligand systems of the metal centre. Figure 15 demonstrates this stoichiometric dependence of the ligand exchange synthetic method. The ligand exchange method is often used in conjunction with CDI insertion, as is the case for this body of work. The 6-coordinate amidinate compound, depicted in Figure 15, is first isolated from a CDI insertion reaction where the carbodiimide inserts across an Al-R bond, where $R$ is an alkyl or amide group. $(49,46,48,47)$

Ligand exchange reactions were used to synthesize compounds 1 and $\mathbf{5}$. When mixing 2 equivalents of tris(diisopropyldimethylguanidinate) aluminum with one equivalent of $\mathrm{AlH}_{3}$, as depicted in Figure 15, three equivalents of compound 1 can be produced. The synthesis of compound 1 was previously reported, however the reaction was said to be complete after allowing the reaction to proceed for 24 hours at room temperature. ${ }^{(46)}$ When trying to reproduce these results it was found that a mixture of products and reactants would always result. The volatility of $\mathrm{AlH}_{3}$ was thought to be the reason behind the failed reactions since the 
ligand exchange reaction is highly dependent on the stoichiometry of the reactants. However, in attempt to alleviate this issue, varying the amounts of excess alane was unsuccessful in pushing the reaction to completion. In order to force the reaction to produce completion, the reaction was performed in a pressure vessel and heated to various temperatures for varying amounts of time. It was determined that heating the reaction at $50^{\circ} \mathrm{C}$ for 24 hours provided enough thermal energy to overcome the energy barrier and produce pure compound 1. Herein, the optimized synthesis of compound 1 with reproducible results is reported.

The ligand exchange reaction between tris(diisopropylacetamidinate) aluminum and $\mathrm{AlH}_{3}$ to produce compound 5 presented problems as the ligand exchange was found to be an equilibrium reaction. By tracking the reaction progress by ${ }^{1} \mathrm{H} N M R$ at room temperature, the point of equilibrium was able to be estimated at 66 hours providing a ratio of 1.15 moles of trisacetamidinate to one mole of compound $\mathbf{5}$. Leaving the reaction to stir at room temperature longer than 66 hour did not produce an increase in compound $\mathbf{5}$, rather a slow decomposition would occur leading to the formation of grey particles in the reaction solution.

As in the case with compound $\mathbf{1}$, heating the reaction in a pressure vessel can be employed to push the reaction to completion. However, with the small amount of decomposition observed at room temperature, any excess thermal energy provided to the reaction would significantly increase the observed decomposition. When attempting the ligand exchange at a reduced temperature, no forward progress was found to occur.

The delicate synthesis of compound 5 produced isolated yields of $16 \%$ when the reaction was halted at the 66 hour equilibrium point. This unfortunate yield was unable to be increased as time would not permit further exploration of alternative synthetic routes. 
However, as will become evident in the characterization section of this thesis, compound $\mathbf{5}$ is the most promising in terms of use as a precursor and deserves further study.

\subsubsection{Air Sensitivity}

Due to aluminum's oxophilic nature, it was imperative that the synthesis of compounds 1-5 be performed under inert atmosphere with anhydrous solvents and reagents. All syntheses and manipulations of the five compounds were conducted in a nitrogen filled glovebox or on a Schlenk line providing either a nitrogen or vacuum environment. Any small leak in the glovebox or Schlenk line set up, or oxygen impurities in reaction solvents lead to the decomposition of reactants and/or products while performing syntheses.

Over the course of this work, certain technical issues arose with the nitrogen filled glovebox which lead to the build up of oxygen in the glovebox atmosphere. Frequent purging of the box atmosphere was not enough to prevent oxygen and/or water from making its way into sealed vials containing the hydride compounds. As difficult as it is to lose compounds due to uncontrolled decomposition, an opportunity to investigate the air sensitivity of the compounds arose.

A vial containing pure compound 3 was slowly infiltrated with oxygen or oxygen containing species, long thin crystals were found to nucleate and grow out from the vial wall. Given enough time, the crystal formations grew the length of the vial as shown in Figure 16. Using ${ }^{1} \mathrm{H}$ NMR and sublimation temperature, the crystal formations were identified as triisopropyl guanidine. Identification of the residual mass in the bottom of the vial, however, 
was not straightforward. A white, fluffy solid was isolated and found to be insoluble in most solvents.

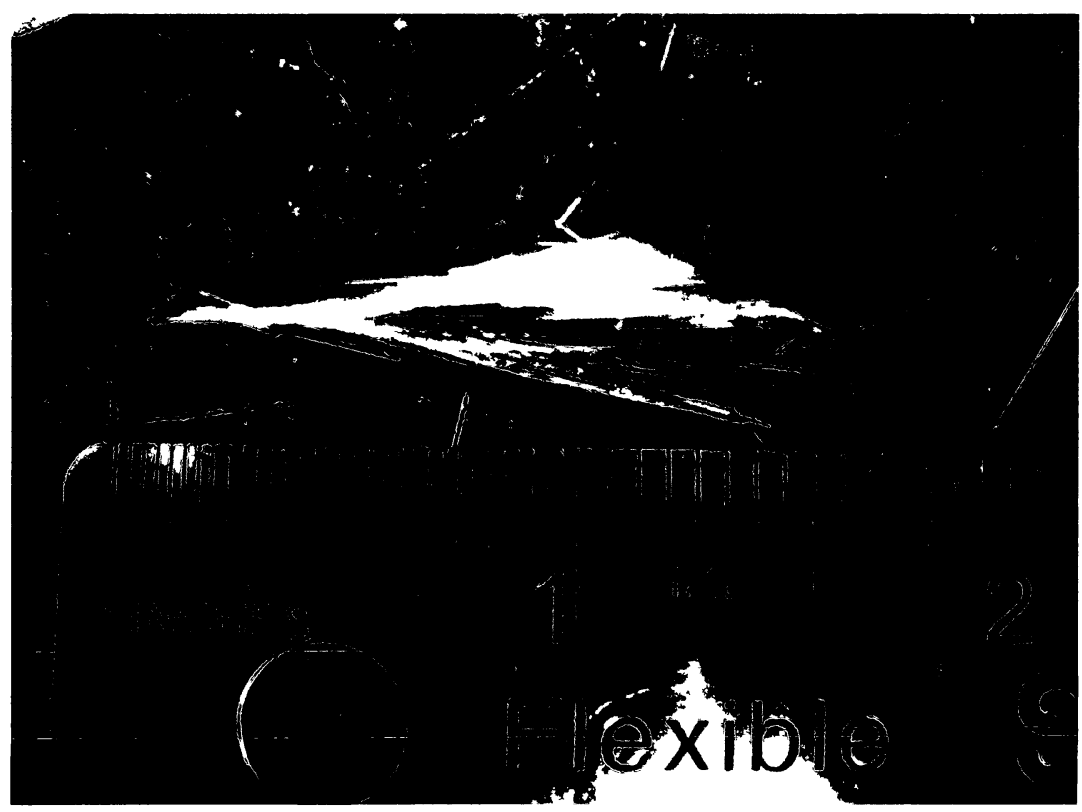

Figure 16. Needle-like crystal formations of triisopropyl guanidine resultant from air assisted decomposition of compound 3

Presumably, the same unknown product resulted from attempts to synthesize compound 3 while the glovebox atmosphere was impure. On a few occasions while performing sublimation of compound $\mathbf{3}$, the formation of triisopropylguanidine needle-like crystals was observed with the unknown product left in the bottom of the apparatus, suggesting air leaks in the sublimation apparatus or Schlenk line. From the data collected, it can be presumed that the exposure of compound 3 to oxygen containing contaminants resulted in decomposition, releasing triisopropyl guanidine, leaving behind a potentially oxo-bridged oligomer product. The loss of guanidine from the aluminum metal centre must result in oligomerization in order to satisfy the +3 oxidation state of aluminum. Although, the full mechanism of decomposition is yet unknown, it is clear that compound $\mathbf{3}$ is extremely air-sensitive. 
Upon removing a sealed vial of pure compound $\mathbf{5}$ from the glovebox and placing it in a fumehood, some interesting results were found. In less than two hours, the ambient atmosphere made its way inside the capped vial resulting in compound decomposition. However, this decomposition was unlike that experienced by compound 3, described above. Instead the formation of circular aluminum metal deposits were observed, shown in Figure 17. The metal deposition clearly originates at point sources potentially defined by crystals of $\mathbf{5}$ and thins out further away from the point source.

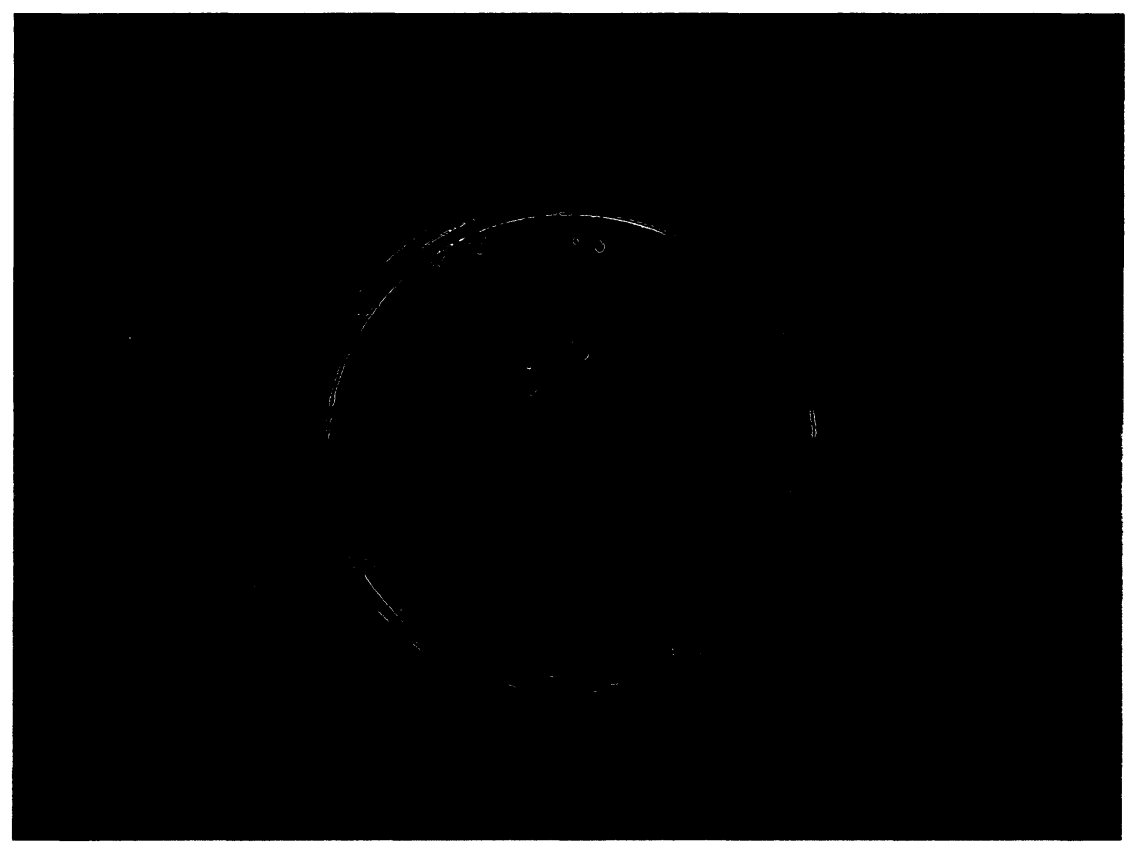

Figure 17. Aluminum metal formations from compound 5 in ambient conditions

When the vial was opened completely to air, no further change was observed. Some white, powdery, solid was found in the vial. The resultant compound was insoluble in several solvents and found to decompose at $\sim 250^{\circ} \mathrm{C} . \mathrm{A}^{1} \mathrm{H}$ NMR of the residual white powder revealed the presence of 6-coordinate trisacetamidinate aluminum as well as other unassigned peaks. 
Since the powder was fairly insoluble, the peaks captured by the ${ }^{1} \mathrm{H}$ NMR represent only the compounds which dissolved, leaving the bulk of the powder unaccounted for.

The deposition of aluminum metal from compound 5 by exposure with ambient air at room temperature is an interesting result. As in the example of ruthenium metal film deposition, the addition of an oxidant can result in a metal film: $\mathrm{Ru}(\mathrm{Cp})_{2}+\mathrm{O}_{2} \rightarrow \mathrm{Ru}^{0}{ }^{(9 ; 50)}$ It is possible that compound 5 may behave similarly. In the case of $\operatorname{Ru}(C p)_{2}$, the ligands were found to be oxidatively decomposed, leaving behind metallic ruthenium. It seems odd to think, that with aluminum's oxophillic nature, the amidinate ligand would oxidatively decompose instead of the aluminum centre becoming oxidized. Also the conditions of the two processes are quite different as the ruthenium deposition process introduces $\mathrm{O}_{2}$ rather than ambient air, where in the aluminum deposition it is more likely that water vapour would interact with the aluminum compound.

It is proposed rather, that the water vapour may have interacted with compound $\mathbf{5}$, which would cause a decomposition releasing thermal energy. This thermal energy could then promote self reduction of compound $\mathbf{5}$ to aluminum metal. The circular aluminum metal formation strongly supports the idea of point source thermal energy. If this self reduction were to occur, the use of compound $\mathbf{5}$ as a single source precursor for CVD of aluminum metal is possible. Of course this hypothesis requires further investigation, but attempts at reproducing the results were unsuccessful. 


\subsection{Characterization}

Preliminary characterization was performed on compounds 1-5 to prove successful synthesis and isolation. ${ }^{1} \mathrm{H} N M R,{ }^{13} \mathrm{C} N M R$, melting point, and electron impact mass spectroscopy data can be found for each compound in the experimental section. A summary of selected preliminary characterization data can be found in Table 2. Included in this characterization chapter are further investigations of the amidinate aluminum hydride family of compounds using single crystal X-ray diffraction ( $s c X R D)$, thermogravimetric analysis (TGA), NMR thermolysis studies, and time-of-flight mass spectrometry (TOF-MS). Through these characterization techniques, valuable information regarding structure and thermal stability is gained.

Table 2. Selected preliminary characterization data for compounds 1-5

\begin{tabular}{ccccc}
\hline Compound & Percent Yield & M.P. & $\boldsymbol{\delta}$ of Al- $\boldsymbol{H}$ & M.S. \\
\hline $\mathbf{1}$ & $85 \%$ & $61-64^{\circ} \mathrm{C}$ & $4.25 \mathrm{ppm}$ & $367 \mathrm{u}$ \\
$\mathbf{2}$ & $86 \%$ & $117-119^{\circ} \mathrm{C}$ & $4.62 \mathrm{ppm}$ & $423 \mathrm{u}$ \\
$\mathbf{3}$ & $93 \%$ & $110-111^{\circ} \mathrm{C}$ & $4.74 \mathrm{ppm}$ & $395 \mathrm{u}$ \\
$\mathbf{4}$ & $78 \%$ & $112-113^{\circ} \mathrm{C}$ & $4.48 \mathrm{ppm}$ & $479 \mathrm{u}$ \\
$\mathbf{5}$ & $16 \%$ & $50-51^{\circ} \mathrm{C}$ & $4.88 \mathrm{ppm}$ & $309 \mathrm{u}$ \\
\hline
\end{tabular}

\subsubsection{Single Crystal XRD}

Of the five compounds synthesized, four crystal structures were able to be collected from crystals grown in toluene at $-30^{\circ} \mathrm{C}$. Structural determination of compounds $\mathbf{1 , 2 , 4}$, and 5 was collected at by the University of Delaware characterization centre using a Bruker AXS APEX diffractometer with a fine-focus sealed tube Mo K- $\alpha$ radiation source $(\lambda=0.71073 \AA)$. The crystals were mounted with viscous oil on a plastic mesh and cooled to the data collection temperature. The structures were solved using direct methods and refined with full matrix least squares 
procedures of $\mathrm{F}^{2}$ data. The optimized structures along with selected geometrical parameters can be seen in Figure 18, 19, 20, and 21, with selected crystal structure data and refinement parameters shown in Table 3. Full '.cif' file data can be found in Appendix A.

Table 3. Selected crystal data and structural refinement parameters for compounds 1, 2, 4, and 5

\begin{tabular}{|c|c|c|c|c|}
\hline & Compound 1 & Compound $\mathbf{2}$ & Compound 4 & Compound 5 \\
\hline fw & 368.55 & 424.38 & 480.76 & 310.46 \\
\hline$T(\mathrm{~K})$ & 206 & 200 & 200 & 200 \\
\hline$\lambda(\AA)$ & 0.71073 & 0.71073 & 0.71073 & 0.71073 \\
\hline cryst syst & Monoclinic & Orthorhombic & Triclinic & Monoclinic \\
\hline space group & $\mathrm{p} 2(1) / c$ & p 2(1) 2(1) 2(1) & P-1 & $\mathrm{C} 2 / \mathrm{c}$ \\
\hline$a(\AA)$ & 13.71 & 10.40 & 9.00 & 11.46 \\
\hline$b(\AA ̊)$ & 9.03 & 15.74 & 18.72 & 12.76 \\
\hline$c(\AA \AA)$ & 19.03 & 17.00 & 19.04 & 14.19 \\
\hline$\alpha$ (deg) & 90 & 90 & 81.06 & 90 \\
\hline$B$ (deg) & 98.11 & 90 & 84.35 & 107.87 \\
\hline$v$ (deg) & 90 & 90 & 89.66 & 90 \\
\hline$V\left(\AA^{3}\right)$ & 2332 & 2783 & 3152 & 1974 \\
\hline$z$ & 4 & 4 & 4 & 4 \\
\hline $\begin{array}{l}\rho \text { (calcd) } \\
\left(\mathrm{Mg} / \mathrm{m}^{3}\right)\end{array}$ & 1.050 & 1.013 & 1.013 & 1.045 \\
\hline abs coeff $\left(\mathrm{mm}^{-1}\right)$ & 0.099 & 0.091 & 0.086 & 0.104 \\
\hline $\begin{array}{l}\text { refinement } \\
\text { method }\end{array}$ & $\begin{array}{l}\text { Full-matrix least- } \\
\text { squares on } \mathrm{F}^{2}\end{array}$ & $\begin{array}{l}\text { Full-matrix least- } \\
\text { squares on } \mathrm{F}^{2}\end{array}$ & $\begin{array}{l}\text { Full-matrix least- } \\
\text { squares on } \mathrm{F}^{2}\end{array}$ & $\begin{array}{l}\text { Full-matrix least- } \\
\text { squares on } \mathrm{F}^{2}\end{array}$ \\
\hline $\begin{array}{l}R \text { indices } \\
{[I>2 \sigma(I)]^{*}}\end{array}$ & $\begin{array}{l}R 1=0.0632 \\
W R 2=0.1408\end{array}$ & $\begin{array}{l}R 1=0.0442 \\
w R 2=0.1195\end{array}$ & $\begin{array}{l}R 1=0.0607 \\
W R 2=0.1450\end{array}$ & $\begin{array}{l}R 1=0.0440 \\
W R 2=0.1286\end{array}$ \\
\hline
\end{tabular}

Interestingly, even though the four compounds are analogous 5 coordinate aluminum species which only differ in the excocyclic moiety of the amidinate ligand, the crystal systems and space groups vary from compound to compound. Compound 1 and $\mathbf{5}$ have monoclinic crystal systems, but their space groups differ as compound $\mathbf{5}$ possesses a C2 axis through the aluminum centre. The triclinic compound $\mathbf{4}$ is even more complicated, as the crystal structure has P-1 symmetry, indicating that the compound does not possess any planes of symmetry, 
rotation axes, and does not possess an inversion centre within the compound; rather the inversion centre exists as a symmetry operation between two structural units. Figures presenting the unit cell of the compounds with views along the $a, b$, and $c$ axis can be found in Appendix A.

When comparing the bond lengths and angles between compounds, the basic structure is fairly similar. The aluminum metal centre always has two shorter bonds and two longer bonds (1.93-1.95 $\AA$ and $2.00-2.06 \AA$, respectively) to the chelating nitrogens of the amidinates. The nitrogens which bind to the aluminum with longer bonds represent the axial positions where as the other two nitrogens and hydrogen provide the equatorial positions. The chelate bite angle of the amidinate ligands appears to decrease with increasing steric bulk within the guanidinates: $67.8^{\circ}, 67.5^{\circ} / 67.4^{\circ}, 67.4^{\circ} / 67.1^{\circ}$ for compound $\mathbf{1}, \mathbf{2}$, and 4 , respectively. As the steric bulk of the excocyclic group in the ligand increases, the nitrogens are forced closer together, essentially decreasing the ligand bite angle. Furthermore, the difference in bite angles between the two ligands of the same compound can be found for compound $\mathbf{2}$ and $\mathbf{4}$. This asymmetry in bite angle can be explained by the bulkier ligand, influencing the extended crystal lattice, causing inequivalence in the intermolecular forces driving the two nitrogens closer together, resulting in different bite angles. 
A)

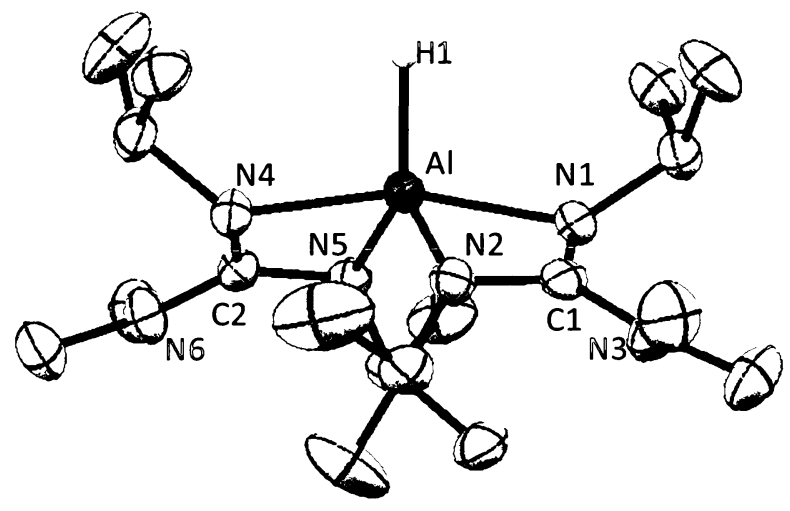

B)

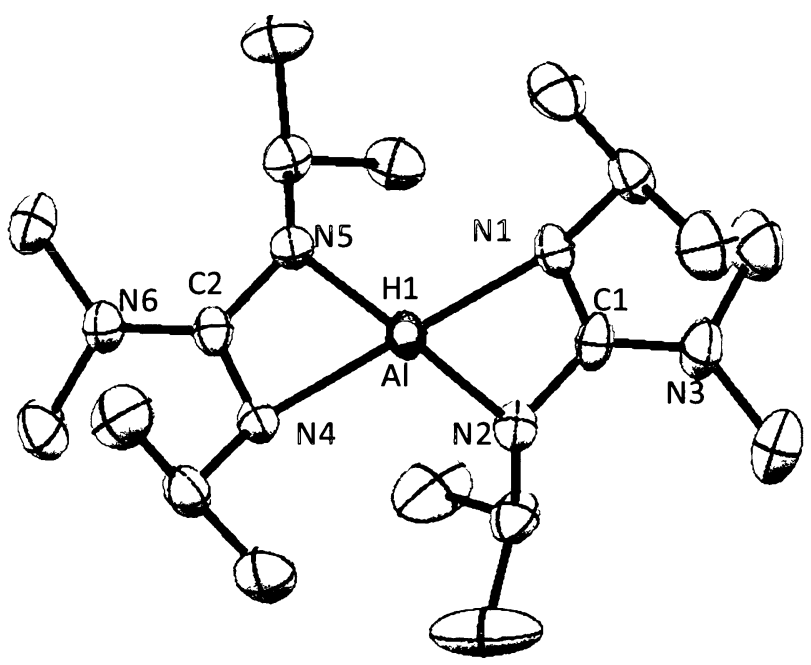

C) Compound 1

\begin{tabular}{lr}
\hline Bond Length (Å) & \\
\hline Al-H1 & 1.58 \\
Al-N1 & 2.00 \\
Al-N2 & 1.95 \\
Al-N4 & 2.00 \\
Al-N5 & 1.95 \\
\hline Bond Angle (deg) & \\
\hline N1-Al-N2 & 67.8 \\
N4-Al-N5 & 67.8 \\
N2-Al-N4 & 102.3 \\
N1-Al-N5 & 102.7 \\
N2-Al-N5 & 116.7 \\
N1-Al-N4 & 162.3 \\
N1-Al-H1 & 100.5 \\
N2-Al-H1 & 122.9 \\
N4-Al-H1 & 97.2 \\
N5-Al-H1 & 120.4 \\
\hline Torsion Angle (deg) & \\
\hline Al-N1-C1-N2 & 0.8 \\
Al-N4-C2-N5 & 1.8 \\
\hline Sum of All Angles (deg) \\
\hline IN3 & 358.9 \\
EN6 & 357.3 \\
\hline & \\
\hline
\end{tabular}

Figure 18. Crystal structure of compound 1 A) view normal to Al-H1, $B$ ) view along $\mathrm{Al}-\mathrm{H1}$, and $\mathrm{C}$ ) selected bond lengths and angles 
A)

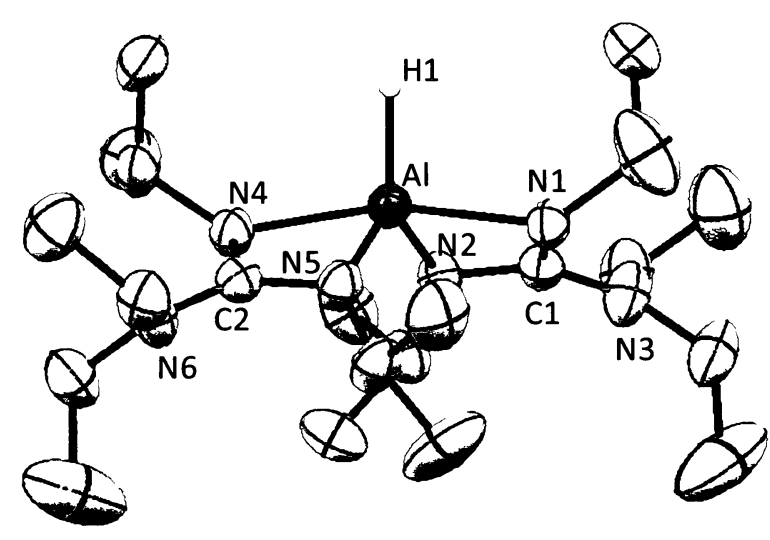

B)

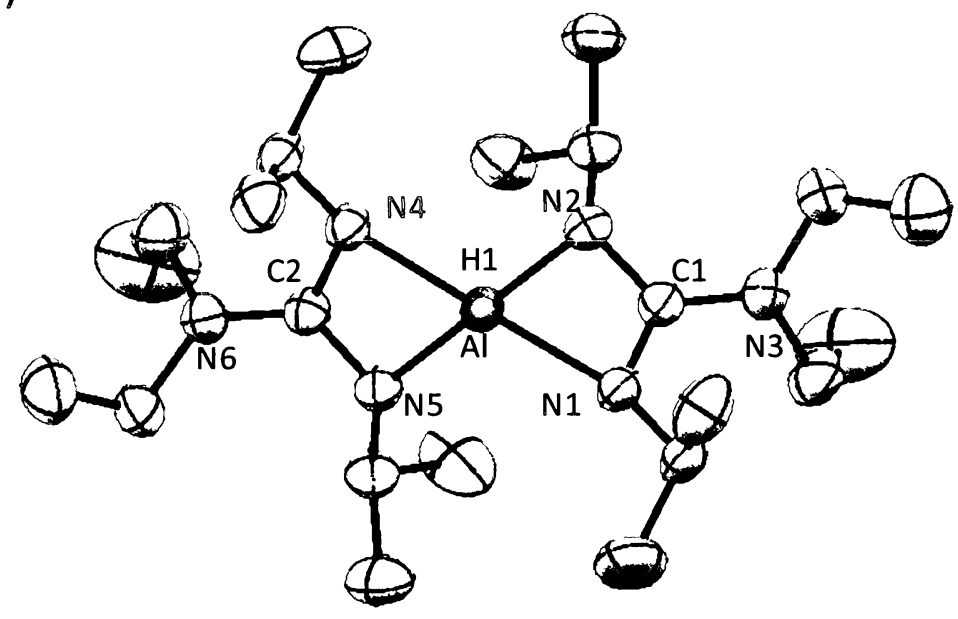

C) Compound 2

\begin{tabular}{lr}
\hline \multicolumn{2}{l}{ Bond Length (Å) } \\
\hline Al-H1 & 1.53 \\
Al-N1 & 2.03 \\
Al-N2 & 1.94 \\
Al-N4 & 2.03 \\
Al-N5 & 1.94 \\
\hline Bond Angle (deg) & \\
\hline N1-Al-N2 & 67.5 \\
N4-Al-N5 & 67.4 \\
N2-Al-N4 & 103.2 \\
N1-Al-N5 & 105.1 \\
N2-Al-N5 & 163.8 \\
N1-Al-N4 & 120.5 \\
N1-Al-H1 & 96.7 \\
N2-Al-H1 & 118.5 \\
N4-Al-H1 & 99.5 \\
N5-Al-H1 & 121.0 \\
\hline Torsion Angle (deg) & \\
\hline Al-N1-C1-N2 & 4.4 \\
Al-N4-C2-N5 & 3.9 \\
\hline Sum of All Angles (deg) \\
\hline EN3 & 358.9 \\
EN6 & \\
\hline & \\
\hline
\end{tabular}

Figure 19. Crystal structure of compound 2 A) view normal to Al-H1, $\mathrm{B})$ view along $\mathrm{Al}-\mathrm{H1}$, and $\mathrm{C}$ ) selected bond lengths and angles 
A)

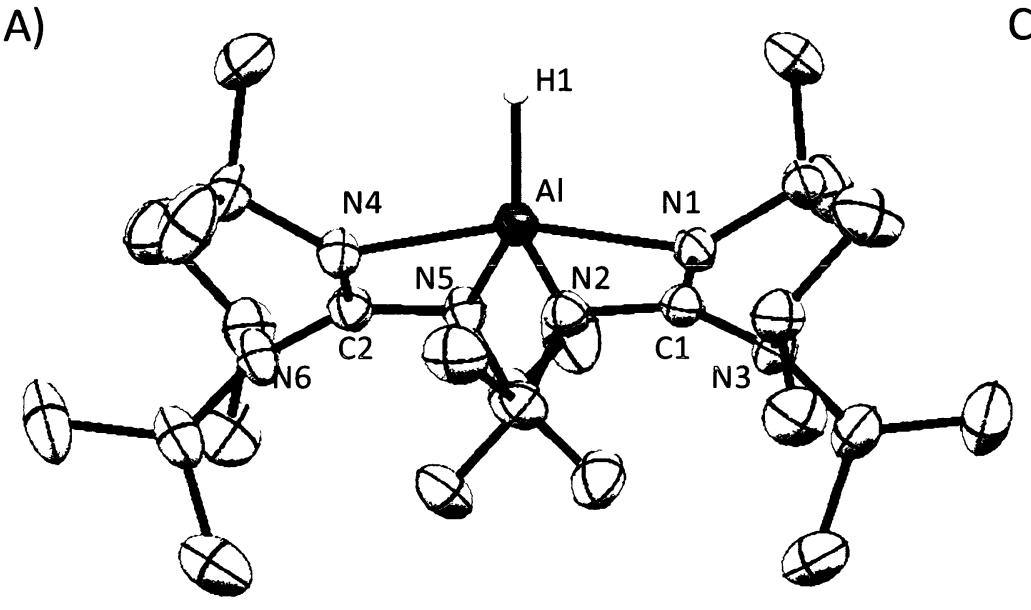

B)

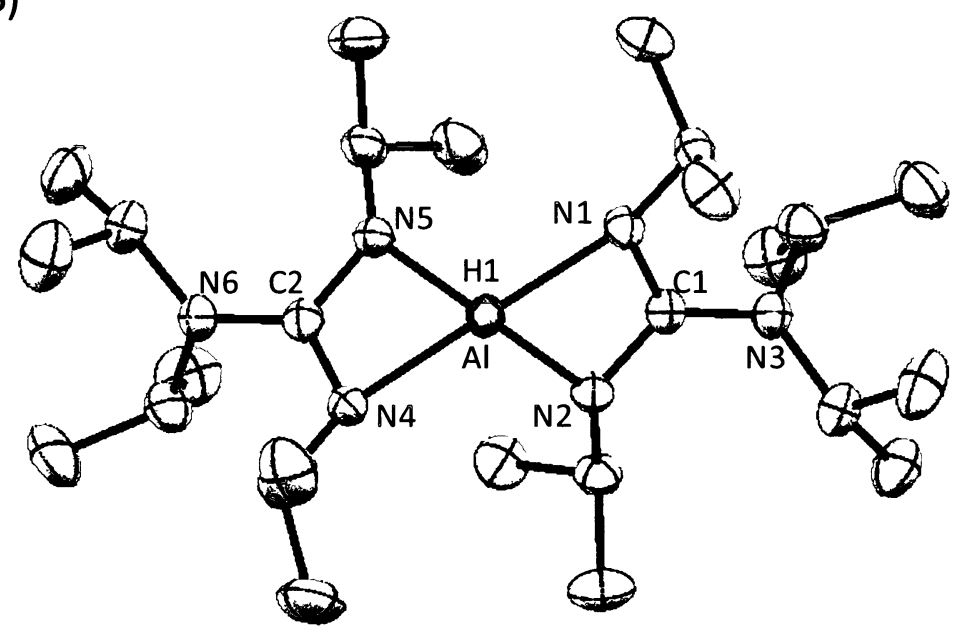

C) Compound 4

\begin{tabular}{lr}
\hline \multicolumn{1}{l}{ Bond Length (Å) } & \\
\hline Al-H1 & 1.51 \\
Al-N1 & 2.03 \\
Al-N2 & 1.93 \\
Al-N4 & 2.06 \\
Al-N5 & 1.93 \\
\hline Bond Angle (deg) & \\
\hline N1-Al-N2 & 67.4 \\
N4-Al-N5 & 67.1 \\
N2-Al-N4 & 103.5 \\
N1-Al-N5 & 102.3 \\
N2-Al-N5 & 161.9 \\
N1-Al-N4 & 117.6 \\
N1-Al-H1 & 98.2 \\
N2-Al-H1 & 119.4 \\
N4-Al-H1 & 99.9 \\
N5-Al-H1 & 123.0 \\
\hline Torsion Angle (deg) & \\
\hline Al-N1-C1-N2 & 358.9 \\
Al-N4-C2-N5 & 357.3 \\
\hline Sum of All Angles (deg) \\
\hline EN3 & \\
EN6 & \\
\hline
\end{tabular}

Figure 20. Crystal structure of compound 4 A) view normal to Al-H1, $\mathrm{B})$ view along $\mathrm{Al}-\mathrm{H1}$, and $\mathrm{C}$ ) selected bond lengths and angles 
A)

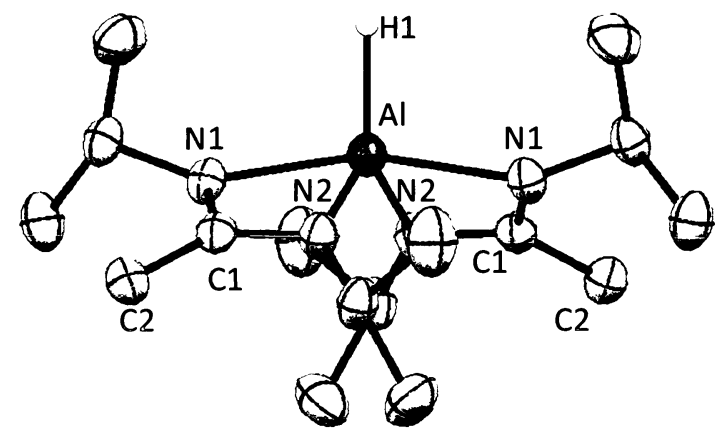

B)

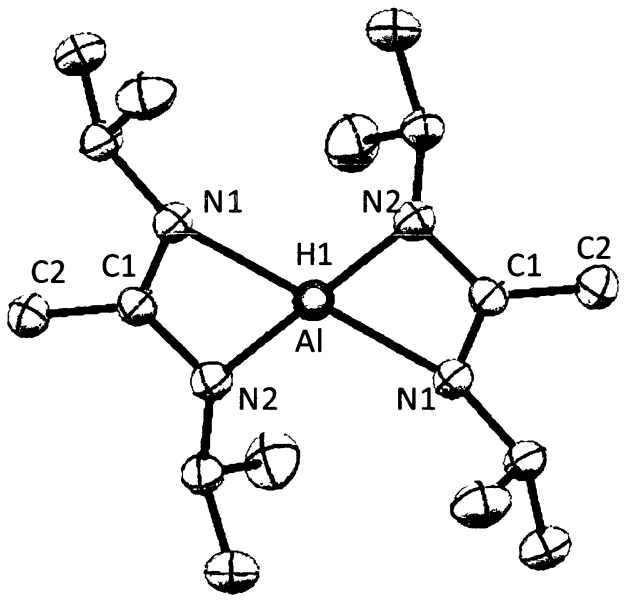

C) Compound 5

\begin{tabular}{lr}
\hline \multicolumn{1}{l}{ Bond Length (Å) } & \\
\hline Al-H1 & 1.94 \\
Al-N1 & 2.06 \\
Al-N2 & 1.94 \\
\hline Bond Angle (deg) & \\
\hline N1-Al-N2 & 66.7 \\
N1-Al-N1 & 103.0 \\
N2-Al-N2 & 161.7 \\
N1-Al-H1 & 99.2 \\
N2-Al-H1 & 122.3 \\
\hline Torsion Angle (deg) & \\
\hline Al-N1-C1-N2 & 1.1 \\
N1-Al-N2-C1 & 0.7 \\
\hline Sum of All Angles (deg) \\
\hline EN3 & 358.9 \\
EN6 & 357.3 \\
\hline
\end{tabular}

Figure 21. Crystal structure of compound 5 A) view normal to Al-H1,

$\mathrm{B})$ view along $\mathrm{Al}-\mathrm{H} 1$, and $\mathrm{C}$ ) selected bond lengths and angles 
In contrast to this trend, the acetamidinate, compound $\mathbf{5}$, exhibits the smallest bite angle of $66.7^{\circ}$. This does not follow the steric bulk trend; one would expect the lack of steric congestion in the exocyclic position to allow the N-C-N ligand backbone to relax in a less perturbed form, resulting in a wider bite angle. However, the opposite is observed and can be attributed to the change in electronic character of the exocyclic position. By switching from an amide group to a methyl group, the excocyclic moiety becomes less electron-donating, resulting in less electron density within the chelate ring, thus allowing for a smaller bite angle when bound to the aluminum centre.

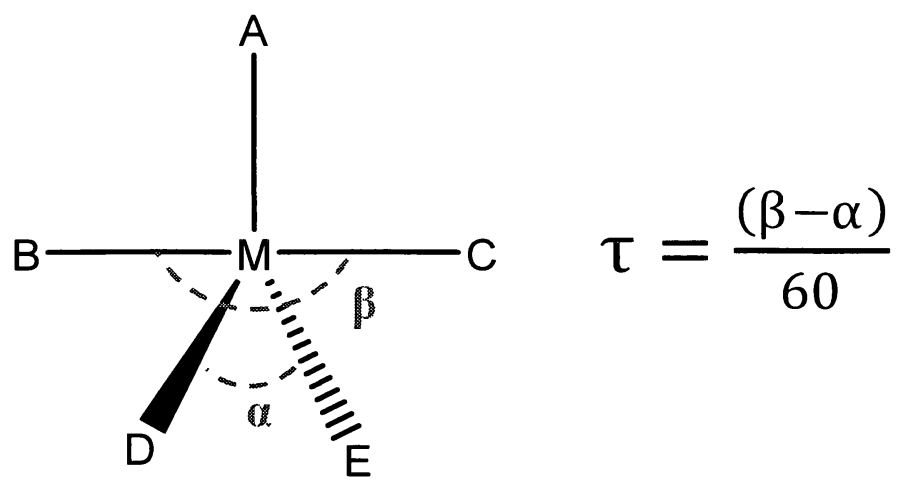

Figure 22. $\tau$ parameter for geometric description of 5-coordinate compounds ${ }^{(51)}$

The geometry of 5-coordinate compounds is often thought of as being either squarepyramidal or trigonal bipyramidal. However, these geometries are idealized and fail to describe compounds which exhibit a certain amount of distortion from the model geometry. Thus, when describing the geometry of 5-coordinate compounds it is helpful to employ a systematic parameter, $\tau$, first described by Addison et. al. as depicted in Figure $22 .{ }^{(51)}$ The parameter $\tau$ allows the geometry of a 5-coordinate compound to be related to square-pyramidal and trigonal bipyramidal geometries, such that when $\tau=0$, the structure is perfectly square- 
pyramidal, and when $\tau=1$, the structure can be describe as being perfectly trigonal bipyramidal. ${ }^{(51)}$

Table 4. Determination of the $\tau$ parameter

\begin{tabular}{cccc}
\hline Compound & $\boldsymbol{\beta}$ & $\boldsymbol{\alpha}$ & $\boldsymbol{\tau}$ \\
\hline $\mathbf{1}$ & $162.3^{\circ}$ & $116.7^{\circ}$ & 0.76 \\
$\mathbf{2}$ & $163.8^{\circ}$ & $120.5^{\circ}$ & 0.72 \\
$\mathbf{4}$ & $161.9^{\circ}$ & $117.6^{\circ}$ & 0.74 \\
$\mathbf{5}$ & $161.7^{\circ}$ & $103.0^{\circ}$ & 0.98 \\
\hline
\end{tabular}

The equation for the $\tau$ parameter selects $\beta$ as the largest of the basal angles and $\alpha$ as the second largest as shown in Figure 22. Thus, using the $\tau$ parameter to help assign geometry to compounds $1,2,4$, and 5, Table 4 demonstrates that all of the guanidinate compounds exhibit behaviour closest to trigonal bipyramidal. The trigonal bypryamidal character of compounds $\mathbf{1}$, 2, and $\mathbf{3}$ is far from perfect as the plane created by N2, N5, and H1 is not perfectly perpendicular to the axial N1, and N4. Instead of the axial positions being located at $90^{\circ}$ to the equatorial plane, the plane is perturbed, resulting in an angle of approximately $100^{\circ}$. This perturbation could be attributed to compression inflicted by the extended crystal structure or bite angle of the ligand.

Table 5. Structural perturbations experienced by compounds 1, 2, 4 and 5

\begin{tabular}{cccccc}
\hline Compound & $\begin{array}{c}\text { Al-H bond } \\
\text { length }(\AA)\end{array}$ & $\begin{array}{c}\text { axial bond } \\
\text { length }(\AA)\end{array}$ & $\begin{array}{c}\text { equatorial } \\
\text { bond length } \\
(\AA \AA)\end{array}$ & $\begin{array}{c}\text { Trigonal } \\
\text { Compression } \\
(\%)\end{array}$ & $\begin{array}{c}\text { Tetragonal } \\
\text { Elongation }{ }^{\text {b }} \\
\text { (\%) }\end{array}$ \\
\hline $\mathbf{1}$ & 1.58 & 2.00 & 1.95 & 2.5 & not defined \\
$\mathbf{2}$ & 1.53 & 2.03 & 1.94 & 4.4 & not defined \\
$\mathbf{4}$ & 1.51 & 2.06 & 1.93 & 6.3 & not defined \\
$\mathbf{5}$ & 1.94 & 2.06 & 1.94 & 5.8 & 0 \\
\hline
\end{tabular}

${ }^{\mathrm{a}} 100(\mathrm{Ax}-\mathrm{Eq}) / \mathrm{Ax},{ }^{\mathrm{b}} 100(\mathrm{Al}-\mathrm{H}-\mathrm{Eq}) / \mathrm{Al}-\mathrm{H}$ 
This trigonal compression can be evaluated using the axial (Ax) and equatorial (Eq) bonds within the compound such that the percentage of compression can be expressed as $100(\mathrm{Ax}-\mathrm{Eq}) / \mathrm{Ax}$. The calculated trigonal compression experienced by compound 1, 2, and $\mathbf{3}$ can be found in Table 6 , clearly demonstrating that as the steric bulk of the excocyclic position increases, so does the amount of trigonal compression experienced. Furthermore, in an ideal trigonal bipyramidal structure, the axial ligands (N1 and N4) would be positioned in line with the metal centre (ie- $\left.<\mathrm{N} 1-\mathrm{Al}-\mathrm{N} 4=180^{\circ}\right) .{ }^{(51)}$ In compounds 1,2 , and 3 , the $<\mathrm{N} 1-\mathrm{Al}-\mathrm{N} 4$ is slightly bent at an angle of approximately $130^{\circ}$, adding to the deviation from perfect trigonal bipyramidal structure.

From the single crystal structures calculated, it is apparent that the Al-N bond lengths within the compounds, as well as the rotations and reflections suggest that there is an inequivalence between the environments of the chelating nitrogens. This is interesting to note since the ${ }^{1} \mathrm{H}$ NMRs collected for the five compounds, as found in the experimental section, present a single doublet peak to represent the isopropyl groups extending from the chelating nitrogens. This single doublet demonstrates that the four isopropyl groups are electronically equivalent.

The equivalence here can be explained through the solution dynamic behaviour that 5coordinate compounds often exhibit, known as Berry pseudorotation. ${ }^{(52)}$ In a solution environment, trigonal bipyramidal compounds undergo fluxional behaviour as shown in Figure 23. This rapid intramolecular exchange occurs as the two axial ligands $(4,5)$, and two of the three equatorial ligands $(2,3)$ move positions through a tetragonal pyramidal transition state, 
resulting in a structure which is a pseudorotation from the original structure. Essentially, the axial ligands become equatorial, and the two equatorial ligands become axial. Since this fluxional behaviour occurs on the time scale of the ${ }^{1} \mathrm{H} N M R$, the ligands are found to exhibit equivalent electronic behaviour.

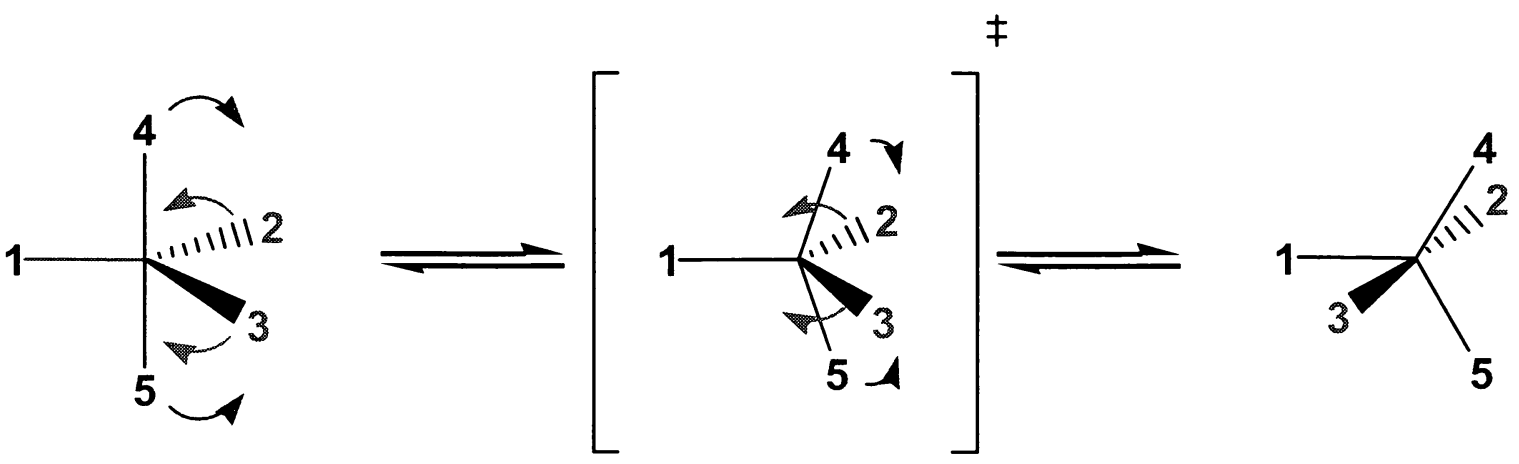

Figure 23. Berry pseudorotation for 5-coordinate compounds

The most noteworthy bond length in this compound with respect to use as an ALD precursor is the $\mathrm{Al}-\mathrm{H}$ bond length. As described within the introduction, a metal hydrogen bond often possesses ionic behaviour such that the hydrogen behaves as an anion. The degree of ionic character of the $\mathrm{M}-\mathrm{H}$ bond is said to be dictated by the ligand environments bound to the metal centre. ${ }^{(36)}$ Thus, the bond length can be related to how strong the interaction between the aluminum and hydrogen is: the longer the bond length, the weaker the interaction, resulting in a more reactive site. The Al-H1 bond lengths for the guanidinates, compounds $\mathbf{1}, \mathbf{2}$, and 4 , are $1.58 \AA, 1.53 \AA$, and $1.51 \AA$, respectively. This trend demonstrates how the decreasing electron donating nature of the exocyclic moiety to the metallocycle (or the increase in steric bulk of the excocyclic moiety) effects the interaction between the aluminum and hydrogen. As the electron density within the metallocycle decreases, the $\mathrm{Al}-\mathrm{H} 1$ bond length increases as related to increasing polarity of the Al- $\mathrm{H}$ bond. When the excocyclic position is occupied by a 
methyl group in compound 5, the Al-H1 bond is lengthen significantly to $1.94 \AA \AA$. This very large $0.4 \AA$ difference in bond length demonstrates how tunable these compounds are with respect to altering the electronic character of the exocyclic position.

The Al-H1 bond length of $1.94 \AA$ can be put into perspective when considering that typical Al-H bonds are about $1.53 \AA$ (as in $\mathrm{AlH}_{3}$ ) and bonds as long as $1.76 \AA$ have been observed for bridging Al-H in $\mathrm{Al}\left(\mathrm{BH}_{4}\right)_{3} .{ }^{(36)}$ Since $\mathrm{M}-\mathrm{H}$ bonds can behave as $\mathrm{M}^{\delta+}--\mathrm{H}^{\delta-}$, the significance of this lengthened $\mathrm{M}-\mathrm{H}$ bond can be translated into the nature of the hydride. This longer Al-H bond in compound $\mathbf{5}$ may prove to be beneficial when considering the potential for surface reactions, provided the Al-H bond can withstand thermolysis of the compound. ${ }^{(36)}$

\subsection{2 ${ }^{1} H$ NMR of Hydrides}

As discussed previously, the ionic nature of the $\mathrm{M}-\mathrm{H}$ bond is completely dependent on the valence orbital energy and surrounding ligand systems of the metal centre. ${ }^{(36)}$ Presumably, the longer the Al-H bond, the more ionic character the bonding will exhibit. Interestingly, when comparing the bond length to the chemical shift of the hydride in Table 7, there is not much correlation. It is true that the analysis methods are performed in very different environments: the Al-H bond length is determined within a crystalline lattice and the electronic/magnetic properties experienced by the hydride is analyzed when in solution. Compound $\mathbf{5}$ does remain an outlier possessing both the longest bond length and largest chemical shift. 
Table 6. Comparison of hydride bond length and chemical shift

\begin{tabular}{ccc}
\hline Compound & $\begin{array}{c}\text { Al-H bond length } \\
(\AA)\end{array}$ & $\begin{array}{c}\boldsymbol{\delta} \text { of Al-H } \\
(\mathbf{p p m})\end{array}$ \\
\hline $\mathbf{1}$ & 1.58 & 4.25 \\
$\mathbf{2}$ & 1.53 & 4.62 \\
$\mathbf{3}$ & - & 4.74 \\
$\mathbf{4}$ & 1.51 & 4.48 \\
$\mathbf{5}$ & 1.94 & 4.88 \\
\hline
\end{tabular}
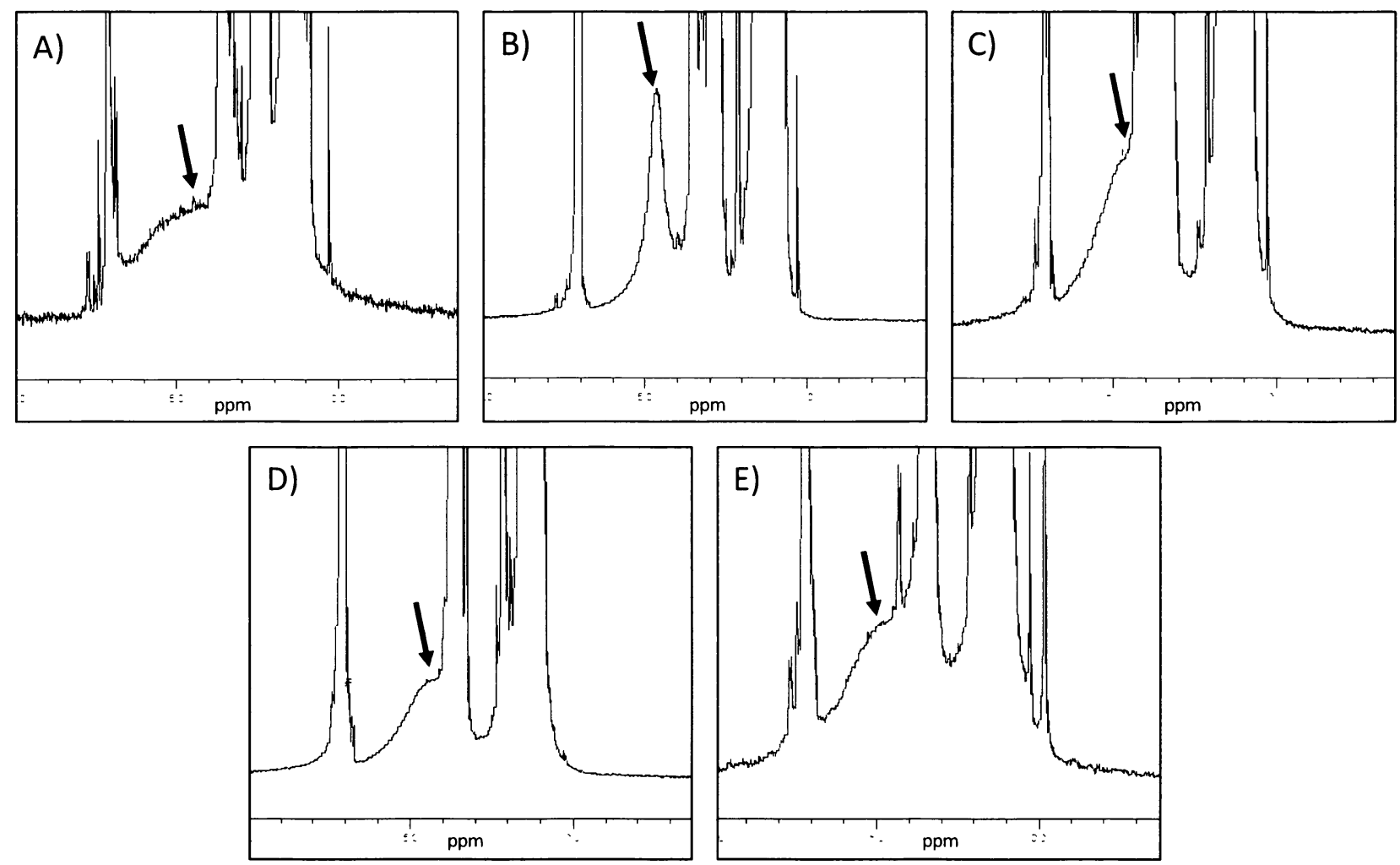

Figure 24. Hydride peak in baseline of ${ }^{1} H$ NMR for A) compound 1, B) compound 2, C) compound 3, D) compound 4, and E) compound 5

In NMR, the further downfield the signal, the more deshielded it is. That is to say, the higher the chemical shift, the more electronegative the proton's attached neighbour is. As the electron density in the ligand systems surrounding the aluminum increase, one would expect the proton to experience more deshielding. Knowing that a hydride is supposed to possess close to anionic behaviour, one would expect a hydride to present far up field, even in the 
negative region. However, the proton peak is found far down field between 4-5ppm, representing an absorption frequency between $1200-1500 \mathrm{~Hz}$. This chemical shift has been found to correlate to that reported in literature, where the chemical shift of hydrido protons are typically reported within the $3.5-5 \mathrm{ppm}$ range. ${ }^{(37,38)}$ The same characteristic broadening is also noted, and is described as being 'presumably' due to coupling with the $\left.A\right|^{27}$ nucleus. ${ }^{(38)} \mathrm{A}$ simple NMR experiment could be performed to verify this assumption by running a ${ }^{1} H$ NMR with aluminum decoupling.

\subsubsection{Thermogravimetric Analysis}

Thermogravimetric analysis (TGA) is a technique in which a sample is loaded into a furnace and heated under an inert atmosphere. The furnace temperature along with the weight of the sample is monitored simultaneously with a $0.1 \mu \mathrm{g}$ weighing precision. All TG data collected, shown in Figures 25-30, were performed on a TGA Q500 by TA instruments with a ramp rate of $5^{\circ} \mathrm{C}$ per minute. Figure 25 provides a direct comparison of compounds $1-5$, as compound weight loss (as a percentage of initial mass) occurs as temperature is increased from room temperature to $550^{\circ} \mathrm{C}$. A few characteristics to be noted of these weight loss curves are the temperature at which the onset of volatility occurs, the slope features of the weight decrease, as well as the residual mass left at the end of the run.

The onset of volatility is defined as the temperature at which the compound has lost $5 \%$ of its initial mass. The onset of volatility is important when selecting a potential precursor for vapour deposition as this demonstrates the relative thermal energy required to volatilize the compound making it available for gas phase deposition. It is important to note that TG is performed close to atmospheric pressure, and thus the temperature required to heat the 
precursor bubbler during depositions is significantly reduced due to low operating pressures. Thus, a comparison of onset of volatility and sublimation temperature, as found in Table 7, is used to determine suitable bubbler temperatures for depositions. Sublimation temperature may appear to reflect the operating conditions of the deposition reactor; however, it fails to provide the accuracy that the TGA offers, as well as the fine detail provided by the subtle changes in slope and the accuracy of residual mass which would be difficult to determine using a bench top sublimation apparatus.

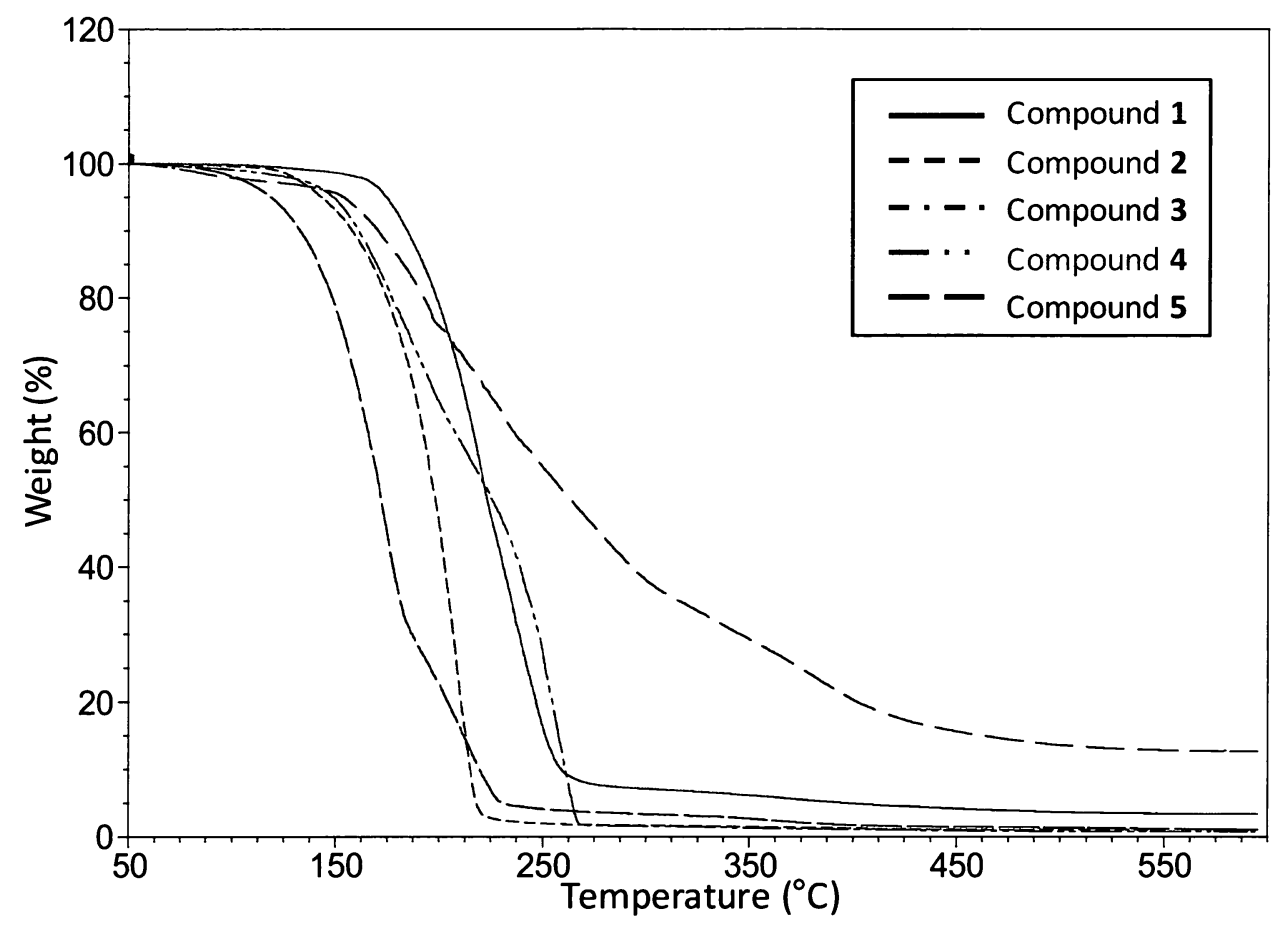

Figure 25. Thermogravimetric analysis of compounds 1-5

It is apparent in Figure 25 that compound $\mathbf{5}$ has the lowest onset of volatility, compound 1 has the highest, and compounds 2-4 have similar results lying directly in between. This trend is similar to the sublimation temperatures and melting points collected for the compounds with the exception of compound 1 . Since the sublimation pressures varied from compound to 
compound, direct comparison of these values could not be made prior to collection of TGA curves. When comparing the onset of volatility to the sublimation temperature it becomes apparent how significant changes in the pressure of the system affect the temperature at which the compound transitions into the gas phase. The sublimation temperature and melting point of compound 1 with respect to the other compounds would suggest a much lower onset of volatility then determined by TGA. This discrepancy can also be elucidated by further analysis using the derivative weight curve in Figure 26.

Table 7. Thermal characteristics of compounds 1-5

\begin{tabular}{ccccc}
\hline Compound & $\begin{array}{c}\text { Melting Point } \\
\left({ }^{\circ} \mathbf{C}\right)\end{array}$ & $\begin{array}{c}\text { Sublimation } \\
\text { Temperature } \\
\left({ }^{\circ} \mathbf{C} / \mathbf{m T o r}\right)\end{array}$ & $\begin{array}{c}\text { Onset of } \\
\text { Volatility } \\
\left({ }^{\circ} \mathbf{C}\right)\end{array}$ & $\begin{array}{c}\text { Residual Mass } \\
(\%)\end{array}$ \\
\hline $\mathbf{1}$ & $61-64$ & $51 / 80$ & 174.4 & 3.36 \\
$\mathbf{2}$ & $117-119$ & $75 / 60$ & 143.3 & 0.97 \\
$\mathbf{3}$ & $110-111$ & $95 / 105$ & 153.5 & 12.6 \\
$\mathbf{4}$ & $112-113$ & $84 / 70$ & 148.9 & 0.76 \\
$\mathbf{5}$ & $50-51$ & $53 / 110$ & 118.8 & 1.01 \\
\hline
\end{tabular}

In order to analyse the subtle changes which occur in the slope of the weight loss curve, the derivative weight curve is added for comparison. Upon taking the derivative of the weight loss curve with respect to temperature, slight changes in the slope of the curve become amplified and more apparent. Figures $26-30$ represent this amplification of subtle features for compounds 1-5. This, in conjunction with the residual mass of the compound can be used to judge the suitability of a compound for use as a precursor for ALD or CVD. Ideal ALD precursors have low onsets of volatilization in conjunction with a single featured, rapid weight loss, and essentially no residual mass. 


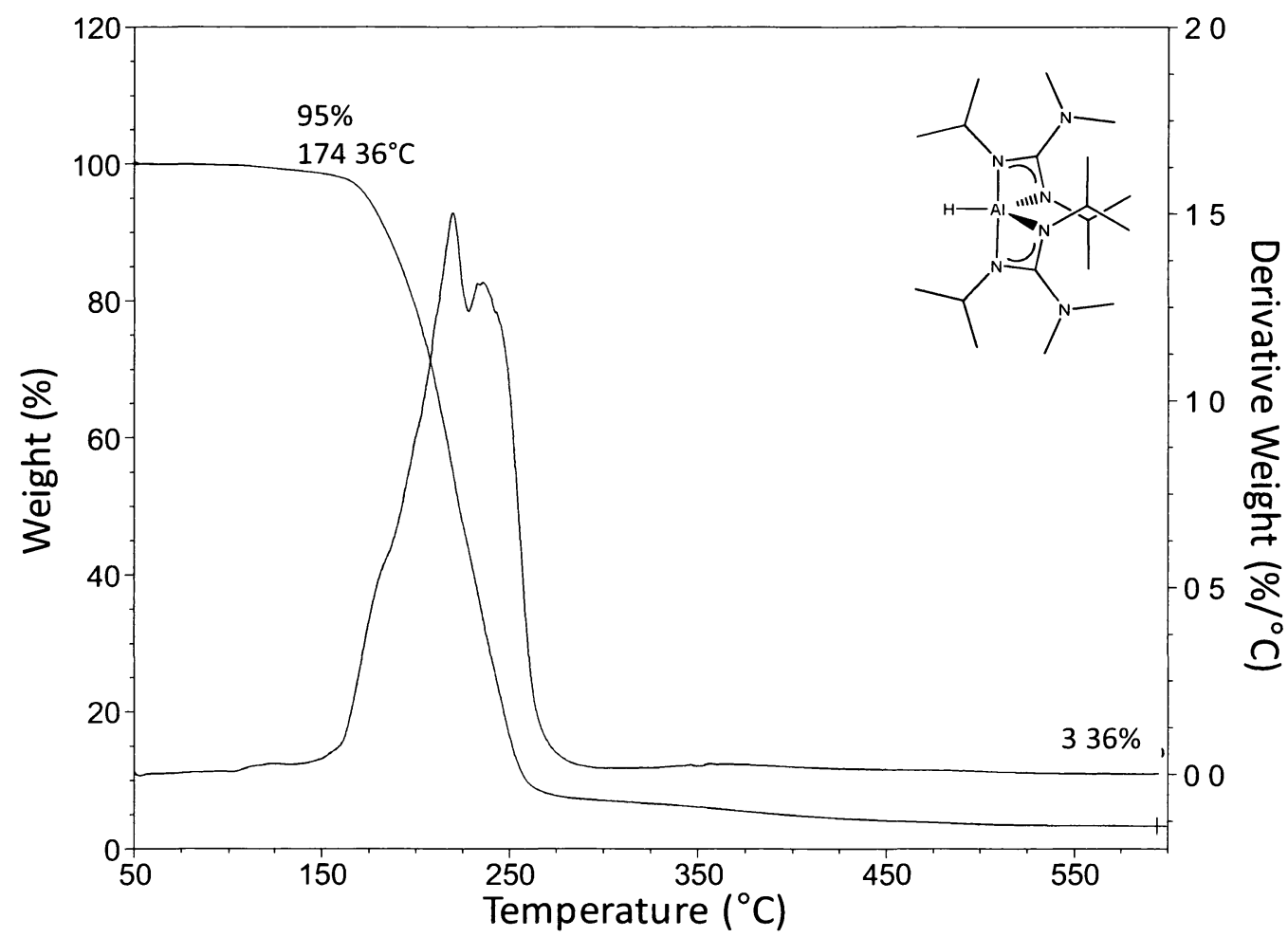

Figure 26. Thermogravimetric analysis of compound 1 with derivative weight curve

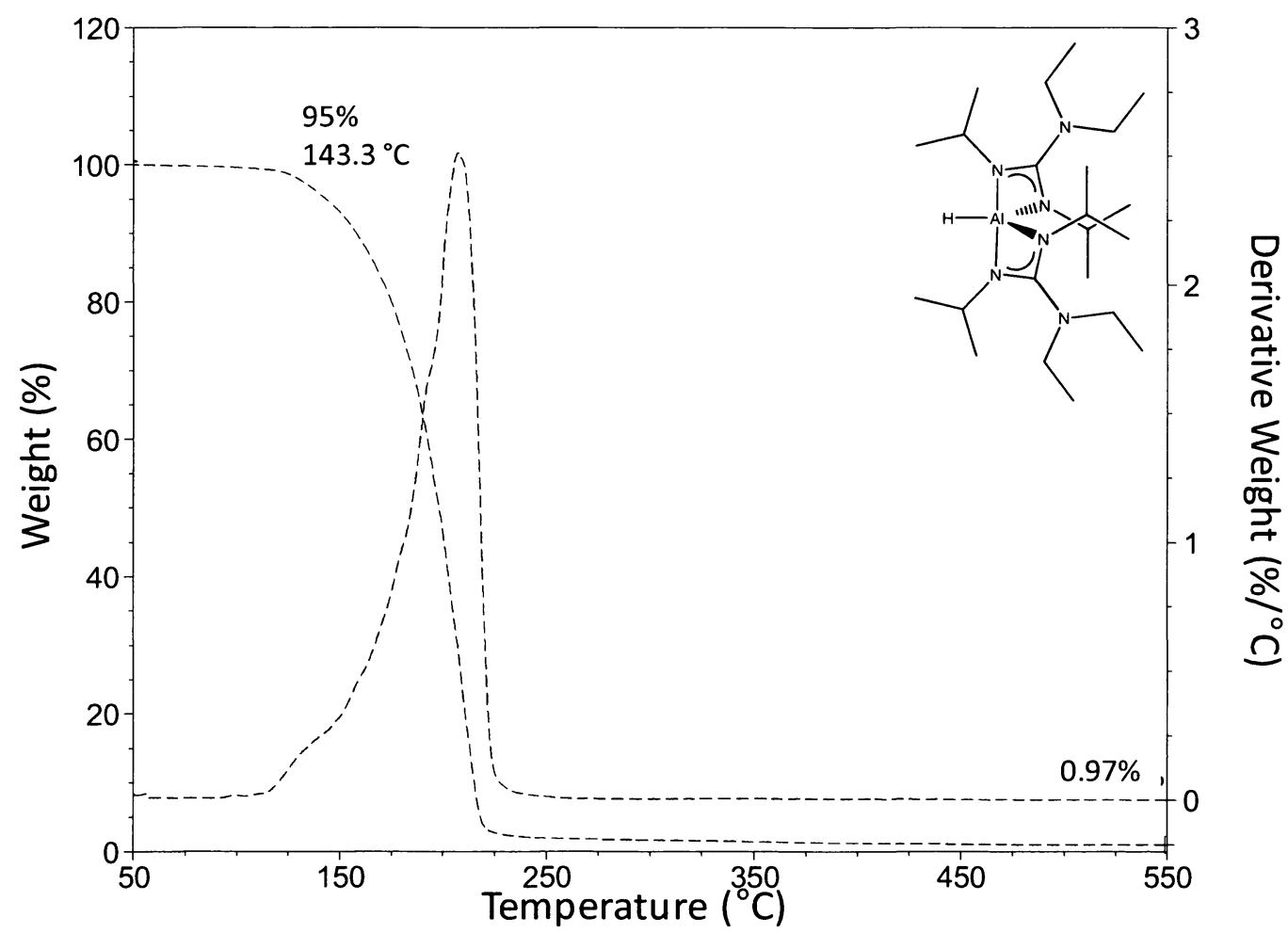

Figure 27. Thermogravimetric analysis of compound 2 with derivative weight curve 


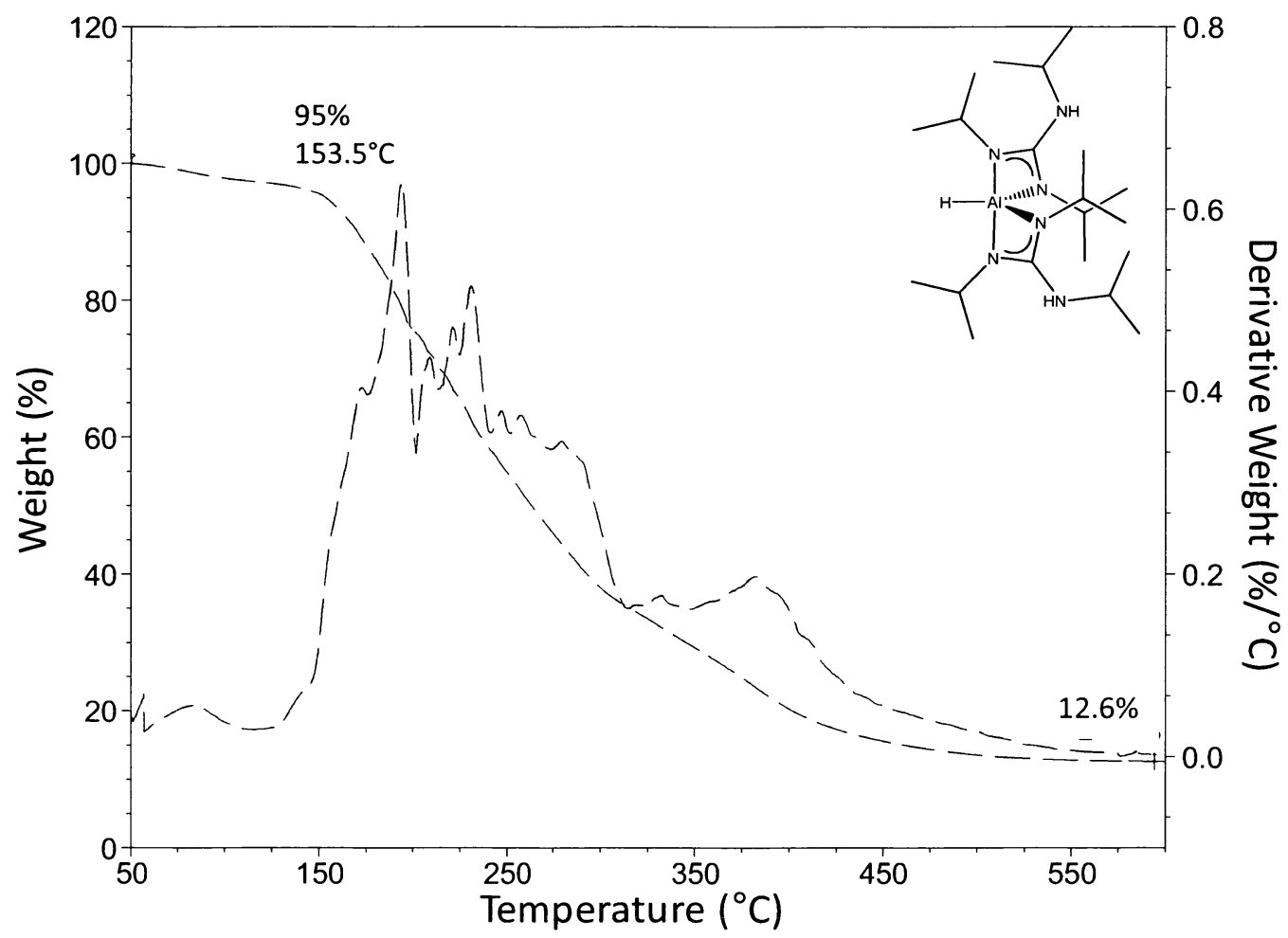

Figure 28. Thermogravimetric analysis of compound 3 with derivative weight curve

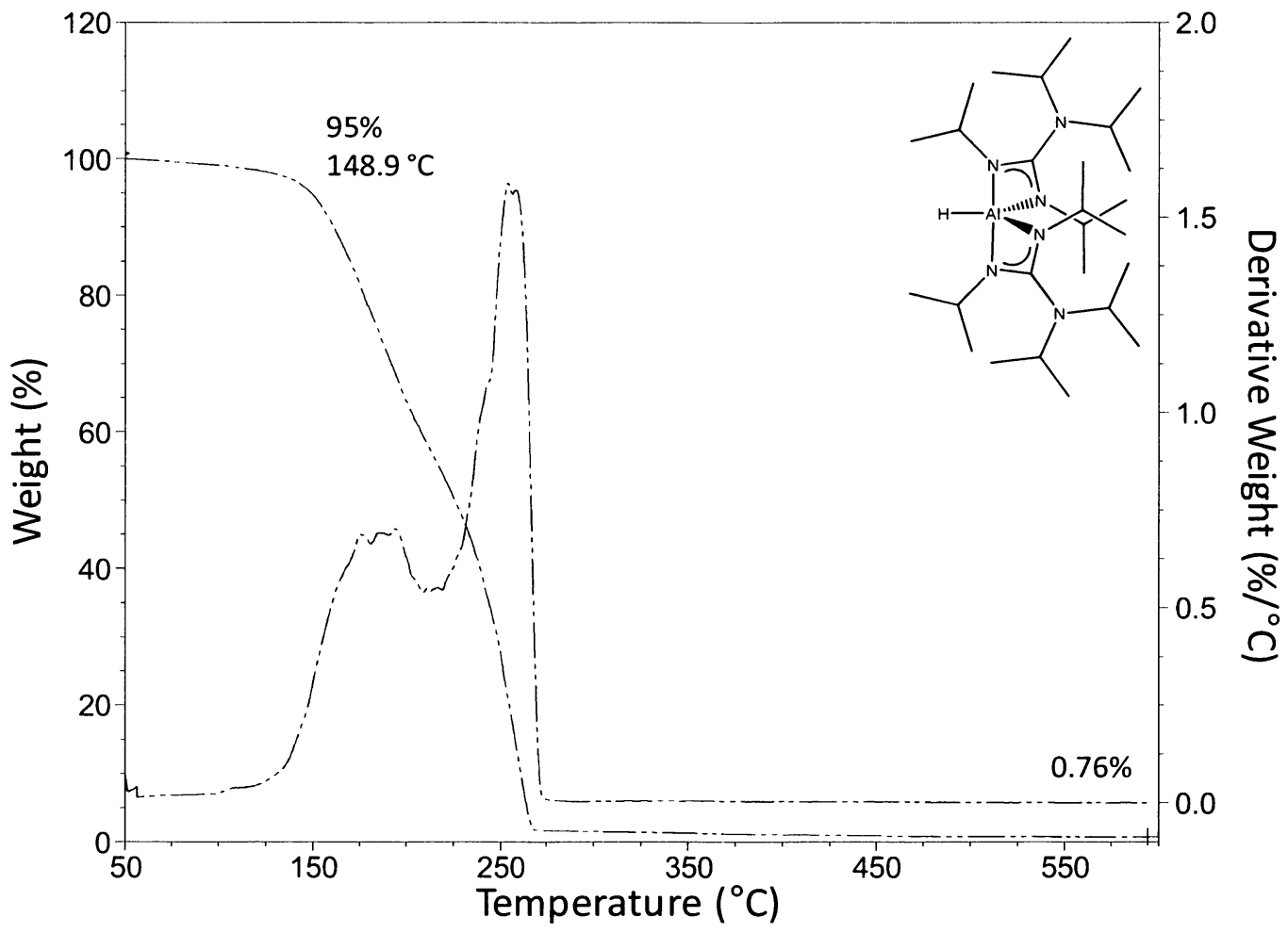

Figure 29. Thermogravimetric analysis of compound 4 with derivative weight curve 


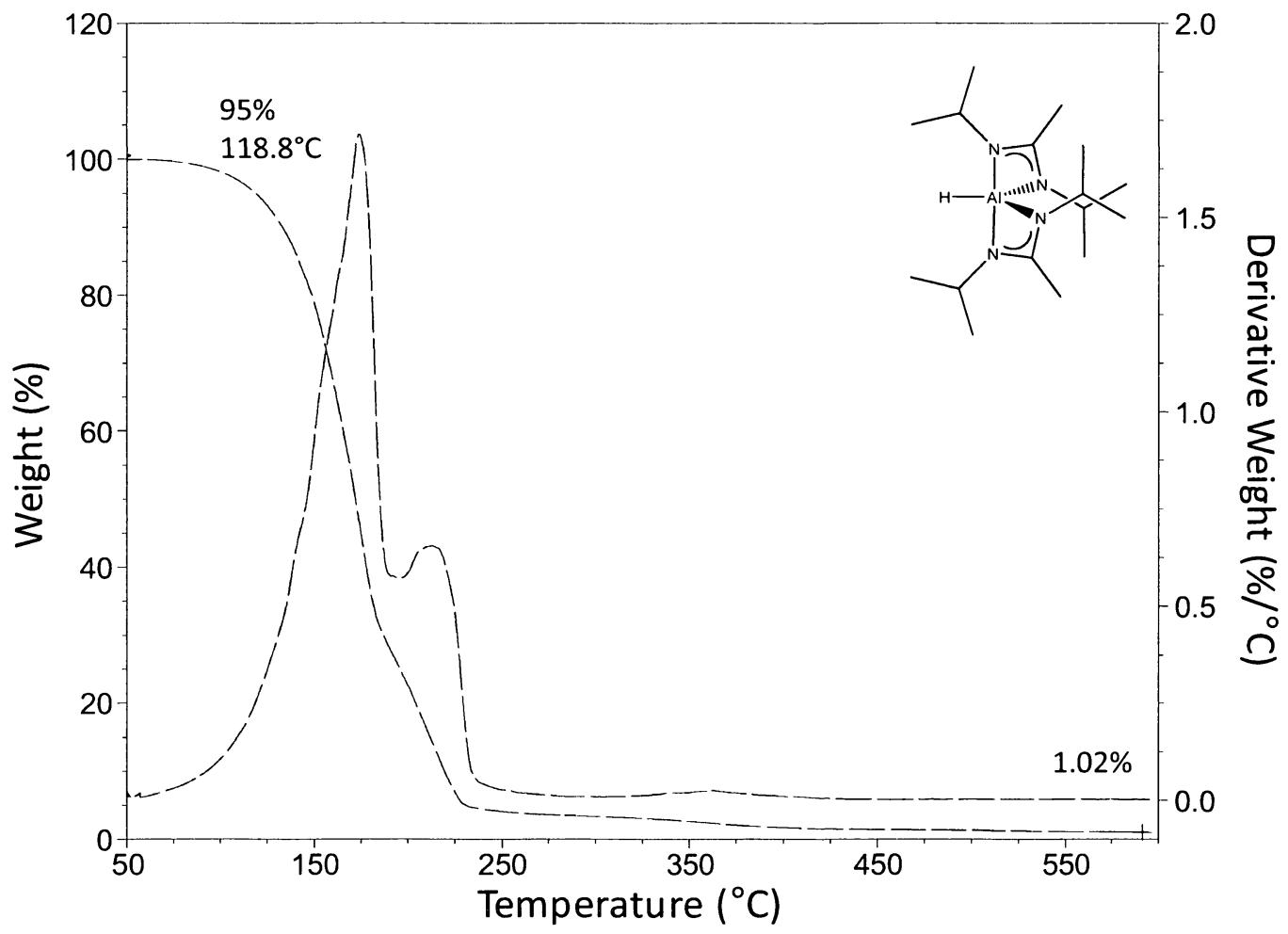

Figure 30. Thermogravimetric analysis of compound 5 with derivative weight curve

The higher than expected onset of volatility for compound 1 can be explained upon analysis of Figure 26. A small feature in the derivative weight loss curve can be seen as a hump at approximately $78^{\circ} \mathrm{C}$. This feature suggests the beginning of volatilization; it is possible that the $5^{\circ} \mathrm{C}$ per minute ramp rate of the TGA experiment may be too fast for the compound to establish an initial temperature of volatilization. The steepness of the weight loss curve between approximately $175^{\circ} \mathrm{C}$ and $250^{\circ} \mathrm{C}$ suggests a rather quick volatilization, however looking at the derivative weight loss curve it is apparent that there are two features to this volatilization suggesting a small amount of thermal decomposition during volatilization. A residual mass of $3.36 \%$ supports the small amount of decomposition which would have produced some non-volatile decomposition products. 
Compound 2, in Figure 27, demonstrates excellent volatility as the derivative weight loss curve is single featured and the temperature window in which this volatilization occurs is fairly narrow between $150^{\circ} \mathrm{C}$ and $225^{\circ} \mathrm{C}$. This, combined with a residual mass of $0.97 \%$, suggests that compound $\mathbf{2}$ is an excellent candidate as an ALD precursor. The $0.97 \%$ residual mass can be attributed to a small level of impurity in the sample and the volatilization of compound 2 can be thought of as complete.

This model TGA for compound $\mathbf{2}$ has an interesting contrast to compound $\mathbf{3}$ in Figure 28. The change in compound weight with increasing temperature for compound $\mathbf{3}$ indisputedly represents thermal decomposition of the compound. An initial hump in the derivative weight loss curve at approximately $75^{\circ} \mathrm{C}$ suggests a possible sublimation window which would explain why using a sublimation apparatus could provide a clean sublimation of the compound (as verified by ${ }^{1} \mathrm{H}$ NMR). If the compound were to be heated to that temperature, then a clean volatilization may be possible, however, with a heating ramp rate of $5^{\circ} \mathrm{C}$ per minute, an apparent complicated thermal decomposition of compound 3 occurs. A residual mass of $12.6 \%$ strongly supports the decomposition of the compound; however this residual mass does not represent the mass of aluminum in the compound $(6.8 \%)$, which could be desirable of an aluminum metal CVD precursor. It is interesting that compound $\mathbf{3}$ presents such a complicated thermal decomposition where the rest of the compounds do not. This can be attributed to the proton in the exocyclic position of the guanidinate ligand, presenting decomposition routes that are not present for the other compounds. 
The residual mass $(0.76 \%)$ left behind after the volatilization of compound 4 would suggest an excellent candidate as an ALD precursor. However, upon analysis of the derivative weight curve in Figure 29, it is evident that the volatilization has two features. The initial peak at approximately $165^{\circ} \mathrm{C}$ depicts a small level of thermal decomposition followed by volatilization represented by the peak at about $250^{\circ} \mathrm{C}$. The TGA of compound $\mathbf{5}$, in Figure 30 , is interesting to compare to that of compound $\mathbf{4}$ as the features of volatilization and decomposition are reversed, and their residual masses are both essentially zero. Instead, an initial volatilization feature at about $175^{\circ} \mathrm{C}$ precedes an element of decomposition at about $220^{\circ} \mathrm{C}$. The order of decomposition and volatilization is extremely important when selecting potential precursors for ALD, as in this case, compound $\mathbf{4}$ would not be suitable. Compound $\mathbf{5}$ on the other hand can be held at volatilization temperature, avoiding the element of decomposition.

Thus, upon Thermogravimetric analysis of compounds 1-5, compound $\mathbf{2}$ and compound 5 can be concluded as being the best candidates for ALD deposition. Both can provide clean sublimation, leaving only impurities behind as residual mass. The temperature at which the onset of volatilization occurs is significantly lower for compound $\mathbf{5}$, making it the best candidate as an ALD precursor.

\subsection{4 ${ }^{1}$ H NMR Thermolysis Studies}

To further explore the thermal decomposition of compounds 1-5, a ${ }^{1} \mathrm{H}$ NMR thermolysis study was performed. Each compound was dissolved in deuterated benzene in a regular NMR tube and sealed under vacuum using a propane torch. The samples were left over night to establish a vapour-liquid equilibrium before commencing the study. An initial ${ }^{1} \mathrm{H}$ NMR spectrum 
was collected for each compound to represent a room temperature sample with no decomposition. ${ }^{1} \mathrm{H}$ NMR spectra were collected periodically to track the thermolysis as the tubes were placed in a furnace and heated. The temperature of the furnace was increased approximately $10^{\circ} \mathrm{C}$ per day until one of the compounds exhibited signs of decomposition. The spectra collected for the duration of the experiment can be found in Appendix B.

At $56^{\circ} \mathrm{C}$, compound 1 and compound $\mathbf{2}$ began to show signs of decomposition as some small peaks were found emerging from the baseline of the spectra collected. The furnace was then held at $56^{\circ} \mathrm{C}$ and ${ }^{1} \mathrm{H}$ NMR spectra were collected for the isotherm until nearly all of compound 1 had converted in decomposition products. Compounds 3-5 remained completely stable at $56^{\circ} \mathrm{C}$, thus, after the decomposition of compound $\mathbf{1}$ had nearly completed, the oven temperature was increased once more to promote decomposition of compounds $3-5$. At $65^{\circ} \mathrm{C}$, compound $\mathbf{3}$ and $\mathbf{4}$ were noted to begin decomposition.

\section{First-order rate law:}

$\frac{d[A]}{d t}=-k[A]$

$$
[A]=[A]_{0} e^{-k t}
$$

\section{Half-life:}

$$
t_{1 / 2}=\frac{\ln 2}{k}
$$

From empirical chemical kinetics we know that the monitoring the molar concentration of a reactant over time can be used to extract a rate constant, $k$. By plotting the natural log of concentration with respect to time, as shown in Figure 30, the slope of the curve provides the rate constant for a first-order reaction. Table 8 provides the rate constants extracted for the 
decomposition of compounds 1-5. The half-life of the compound, $t_{1 / 2}$, was calculated using equation 3, and can also be found in Table 8.

Figure $31 \mathrm{~A}$ ) presents the data collected for compounds 1 and 2 for the isotherm at $56^{\circ} \mathrm{C}$ and Figure 31B) presents the data collected for compounds 3-5 for the isotherm at $65^{\circ} \mathrm{C}$. Using benzene as an internal standard, the ratio of a peak representing protons on the compound with respect to integration of a peak representing the residual protons on the benzene provides us with a ratio that is analogous to the concentration of compound in benzene. Compound $\mathbf{5}$ was completely stable for the duration of the isotherm at $65^{\circ} \mathrm{C}$ and was found stable at higher temperatures. Unfortunately this method has a limitation with respect to the temperature of the benzene: the data collected is only dependable up until the boiling point of benzene $\left(80^{\circ} \mathrm{C}\right)$.

From the data collected, it is quite evident that the relative thermal stability of compounds $1-5$ in solution is $1<2<4<3<5$. However, when comparing this result to the TGA data collected, the result is quite different. TGA determined that compounds $\mathbf{2}$ and $\mathbf{5}$ were found to have the best thermal stability and compounds $\mathbf{1}$ and $\mathbf{3}$ demonstrated the most decomposition upon thermolysis. The discrepancies between these two thermolysis methods is quite simple; the ${ }^{1} \mathrm{H}$ NMR thermolysis method is solution based, thus the reactivity found using this method does not directly model the stability of the compound during deposition techniques. What this method does allow is the exploration of weak points in the compound and associate potential decomposition mechanisms that the compound may experience. 


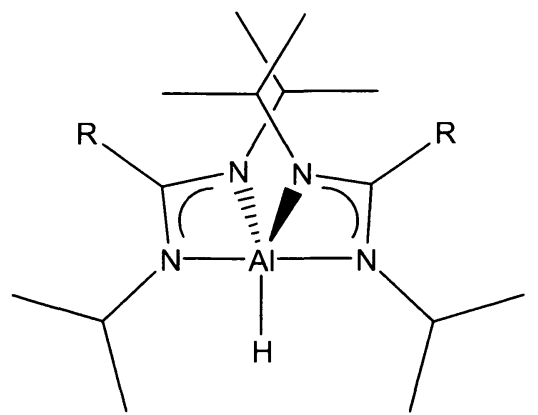

Table 8. Kinetic data collected for compounds 1-5

\begin{tabular}{ccccccc}
\hline Symbol & Compound & $\mathbf{R}$ & $\begin{array}{c}\text { Isothermal } \\
\text { Temperature }\end{array}$ & $\begin{array}{c}\text { Rate } \\
\text { Constant, } \mathbf{k}\end{array}$ & $\begin{array}{c}\text { Calculated } \\
\text { Half-Life, } \\
\mathbf{t}_{\mathbf{1} \mathbf{2}}\end{array}$ & $\begin{array}{c}\text { Correlation } \\
\text { Coefficient, } \\
\mathbf{R}^{\mathbf{2}}\end{array}$ \\
\hline $\mathbf{n}$ & $\mathbf{1}$ & $\mathrm{NMe}_{2}$ & $56^{\circ} \mathrm{C}$ & $4.4 \pm 0.2 \times 10^{-6} \mathrm{~s}^{-1}$ & 1.8 days & 0.98 \\
\hline & $\mathbf{2}$ & $\mathrm{NEt}_{2}$ & $56^{\circ} \mathrm{C}$ & $1.07 \pm 0.04 \times 10^{-6} \mathrm{~s}^{-1}$ & 7.5 days & 0.99 \\
\hline- & $\mathbf{3}$ & $\mathrm{N}^{\prime} \mathrm{Pr}, \mathrm{H}$ & $65^{\circ} \mathrm{C}$ & $1.49 \pm 0.02 \times 10^{-6} \mathrm{~s}^{-1}$ & 5.4 days & 1.00 \\
$\mathbf{\Delta}$ & $\mathbf{4}$ & $\mathrm{N}^{\prime} \mathrm{Pr}_{2}$ & $65^{\circ} \mathrm{C}$ & $1.66 \pm 0.07 \times 10^{-6} \mathrm{~s}^{-1}$ & 4.8 days & 0.99 \\
$\mathrm{X}$ & $\mathbf{5}$ & $\mathrm{Me}$ & $65^{\circ} \mathrm{C}$ & $\mathrm{N} / \mathrm{A}$ & $\mathrm{N} / \mathrm{A}$ & $\mathrm{N} / \mathrm{A}$ \\
\hline
\end{tabular}

A)

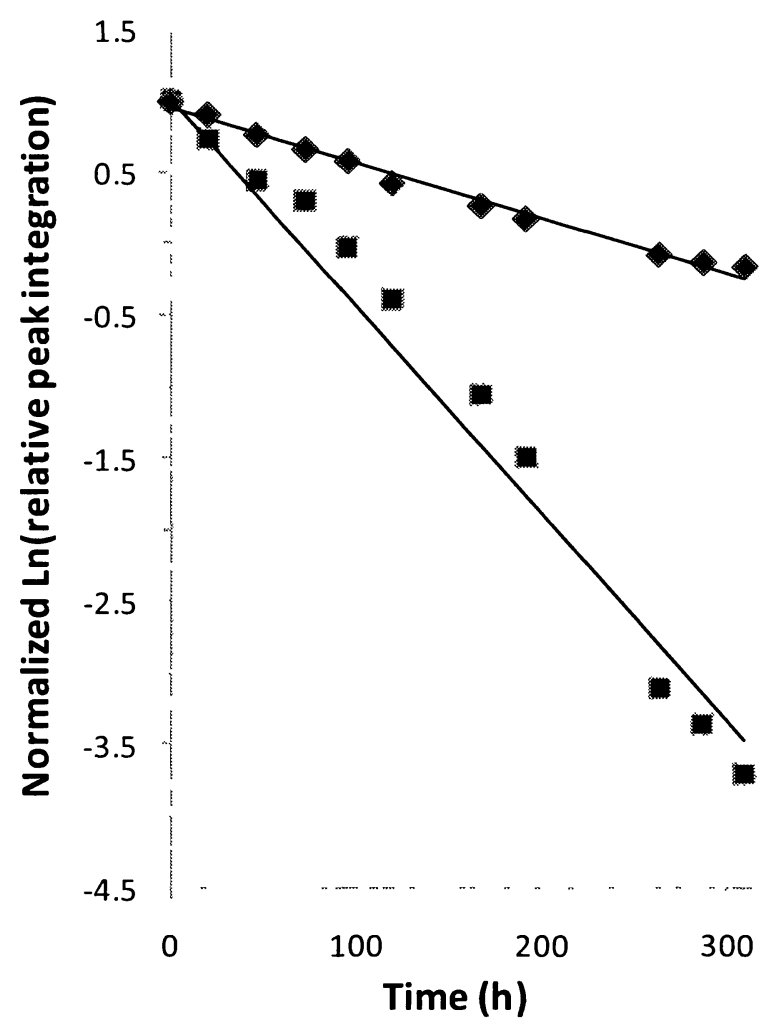

B)

\section{5}

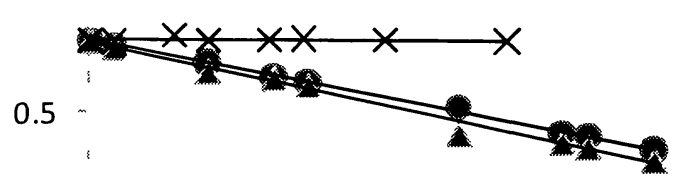

$-0.5$

$-1.5$

$-2.5$

$-3.5$

$-4.5$

$$
50 \text { Time (h) }
$$

Figure 31. ${ }^{1} \mathrm{H}$ NMR Boilup data collected for the decomposition of A) compounds 1 and 2 at $56^{\circ} \mathrm{C}$, and B) compounds 3,4 , and 5 at $65^{\circ} \mathrm{C}$ 
The benefit to monitoring the decomposition of a compound using ${ }^{1} H N M R$, is that decomposition products can often be identified. Fortunately, the decomposition of compound 1 resulted in products with known spectra which were able to be identified by matching peak chemical shifts. Figure 32 is a plot of the decreasing concentration of compound 1 at $56^{\circ} \mathrm{C}$ with increasing concentration of three by-products. The three by-products found to grow into the spectra over the course of the experiment were able to be identified as the structures shown in Figure 33. With known compounds, it was possible to normalise the peaks growing into the spectra allowing for the plot in Figure 32 to demonstrate the molar ratio of the parent compound to that of the decomposition products. Decomposition products $\left[\mathrm{Me}_{2} \mathrm{NC}\left(\mathrm{N}^{\prime} \mathrm{Pr}\right)_{2}\right] \mathrm{Al}\left(\mathrm{NMe}_{2}\right)_{2},\left[\mathrm{HC}\left(\mathrm{N}^{\prime} \mathrm{Pr}\right)_{2}\right]_{2} \mathrm{AlH}$, and $\left[\mathrm{HC}\left(\mathrm{N}^{\prime} \mathrm{Pr}\right)_{2}\right]_{3} \mathrm{Al}$ plotted in Figure 32 account of $98 \%$ of the parent compounds decomposition. Figure 33 depicts a fourth decomposition product, $\left[\mathrm{Me}_{2} \mathrm{NC}\left(\mathrm{N}^{\prime} \mathrm{Pr}\right)_{2}\right]_{2} \mathrm{Al}\left(\mathrm{NMe}_{2}\right)$, which was present among a few other minor peaks in the spectra.

The formation of formamidinate compounds as decomposition products was somewhat surprising considering they are the much more susceptible to CDI deinsertion when compared to acetamidinates and guanidinates. ${ }^{(26)}$ In order to explain the formation of the four identified decomposition products, Figure 34 presents a proposed mechanism. Although deinsertion of CDI is a well known decomposition pathway for amidinate and guanidinate compounds and has been well studied, this decomposition is believed to be the first known instance of a rapid reinsertion. ${ }^{(25,26,27,28)}$ It is believed that the thermolysis of compound 1 provided the required thermal energy to overcome an energy barrier similar to that describe by previous theoretic studies. $^{(25)}$ 


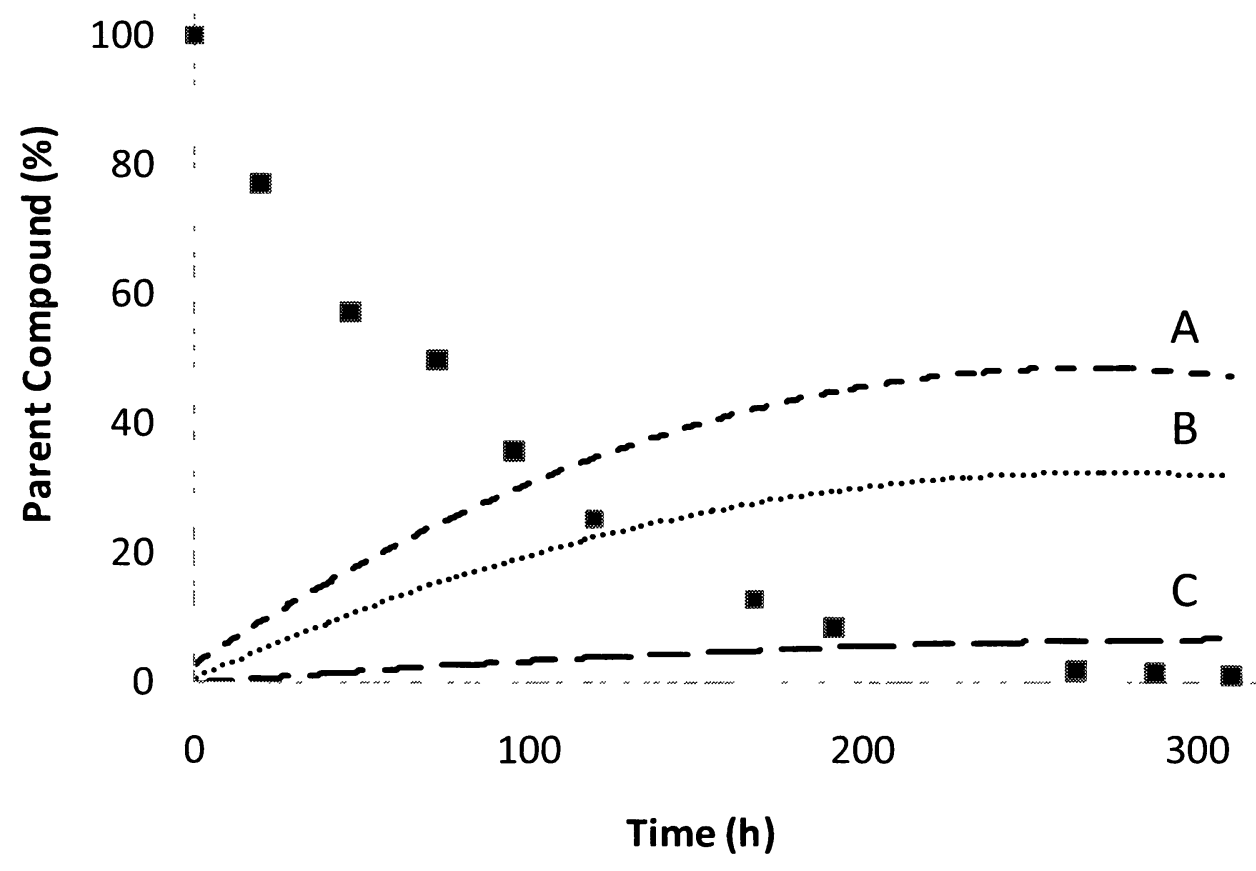

Figure 32. Decomposition of compounds $1(\mathrm{a})$ at $56^{\circ} \mathrm{C}$ into products $\left[\mathrm{Me}_{2} \mathrm{NC}\left(\mathrm{N}^{\mathrm{P} P r}\right)_{2}\right] \mathrm{Al}\left(\mathrm{NMe}_{2}\right)_{2}(--),\left[\mathrm{HC}\left(\mathrm{N}^{\mathrm{i} P r}\right)_{2}\right]_{2} \mathrm{AlH}(\ldots)$ and $\left[\mathrm{HC}\left(\mathrm{N}^{\prime} \mathrm{Pr}\right)_{2}\right]_{3} \mathrm{Al}(--)$.

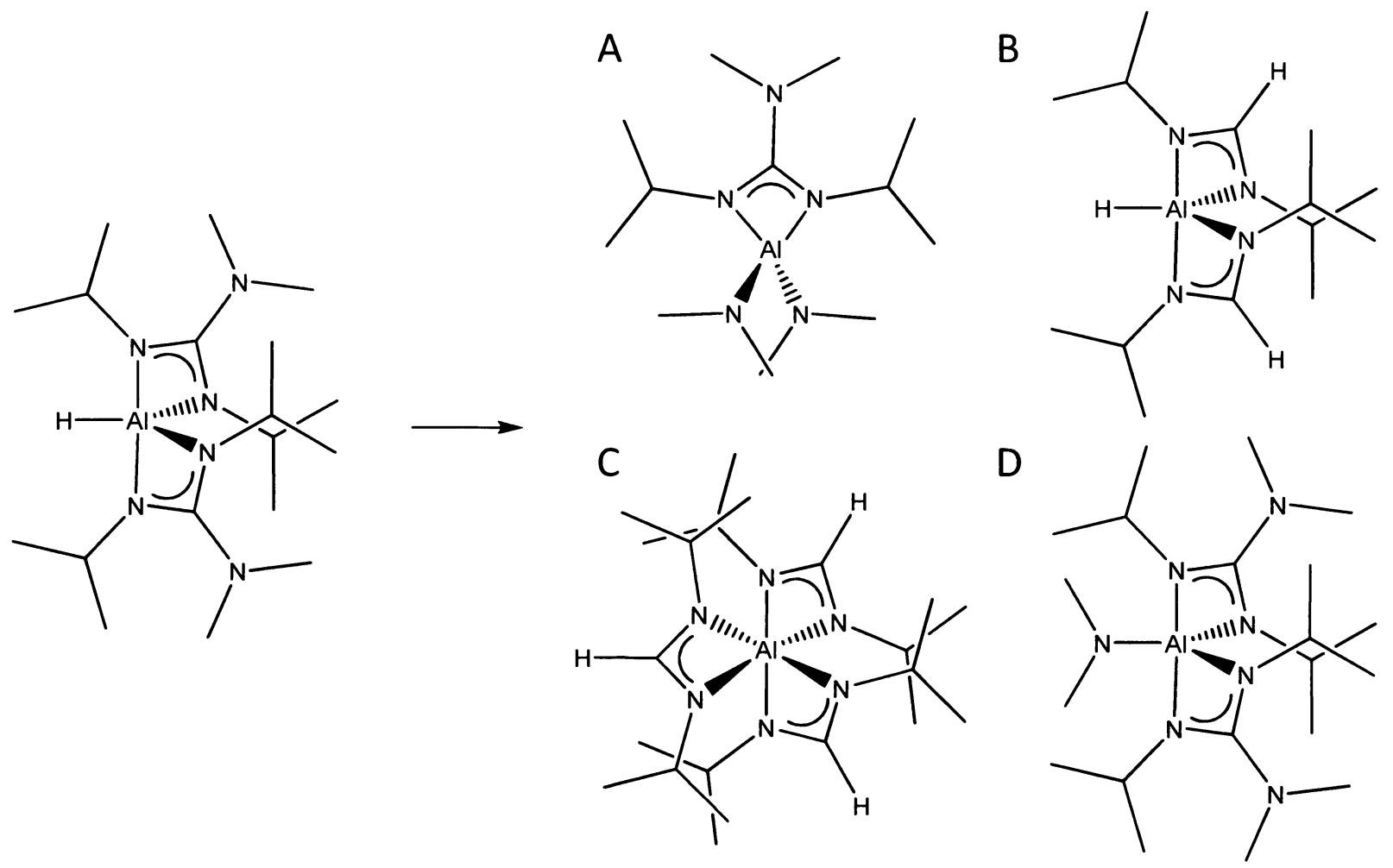

Figure 33. Decomposition products of compounds 1 at $56^{\circ} \mathrm{C}$ 

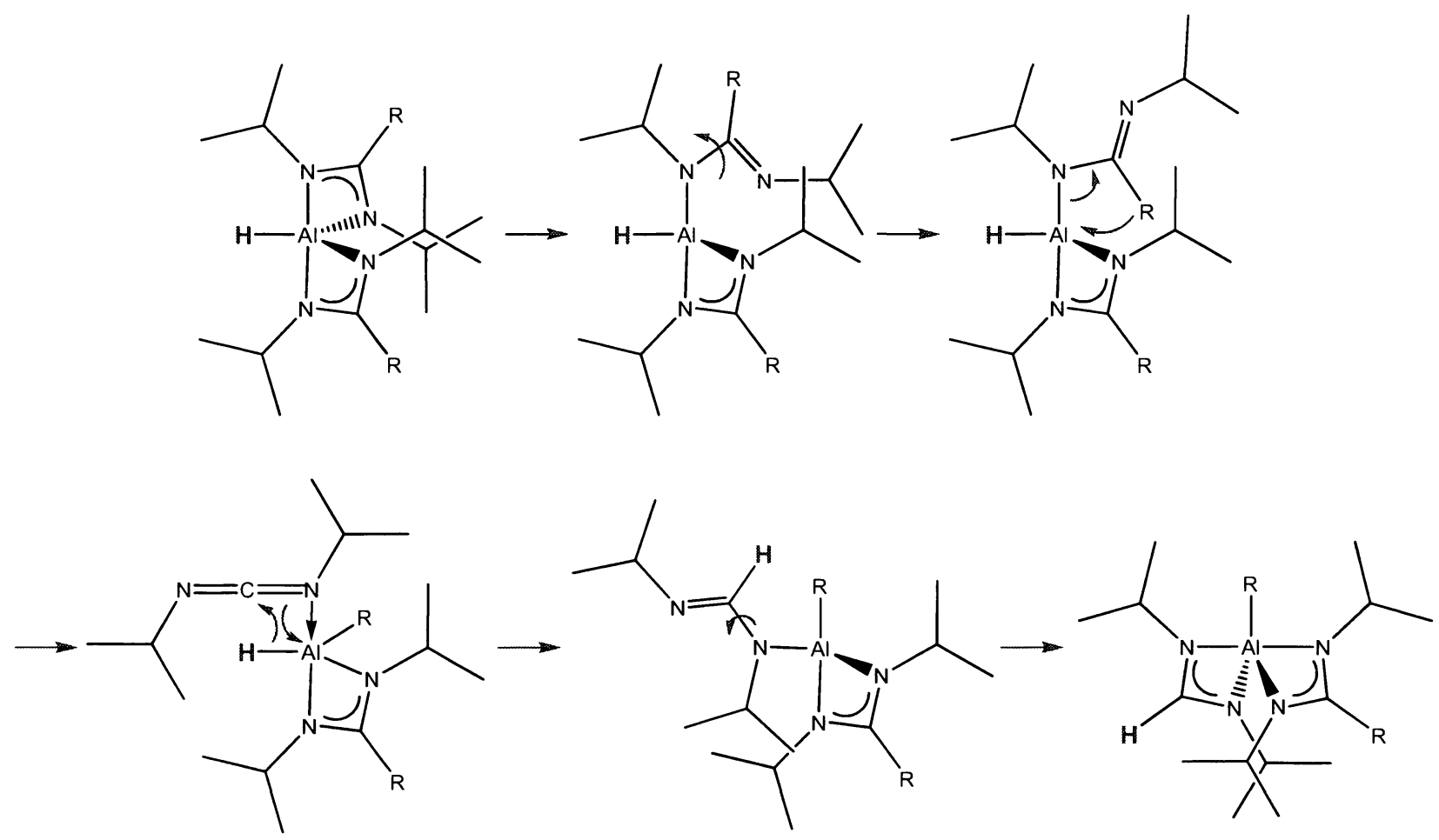

Figure 34. Proposed decomposition mechanism of guanidinate aluminum hydrides based on thermolysis of compound 1

The release of the datively bound nitrogen from the aluminum centre allows for a rotation to occur along the $\mathrm{N}-\mathrm{C}$ bond, bringing the exocyclic moiety into proximity of the aluminum. The aluminum then preferentially binds to the exocyclic amide, depicted in Figure 34 as ' $R$ ', resulting in formal production of the ' $\mathrm{Pr}$-CDI. Traditionally, this ' $\mathrm{Pr}$-CDI is found to exist in the ${ }^{1} \mathrm{H}$ NMR as an independent species. However, the absence of 'Pr-CDI peaks growing in to the ${ }^{1} \mathrm{H}$ NMR during the thermolysis suggests that the $\mathrm{Pr}-\mathrm{CDI}$ immediately reinserts across the Al-H bond, thus explaining the presence of formamidinate species in the ${ }^{1} \mathrm{H}$ NMR.

The reason why this reinsertion has not been previously observed is fairly simple; these amidinate aluminum hydrides are unique in that the $\mathrm{Al}-\mathrm{H}$ bond is much weaker than the Al-R 
bond which would result during 'Pr-CDI deinsertion. The fact that these compounds can be synthesized and stored at room temperature without significant decomposition is completely dependent on the activation energy required to deinsert 'Pr-CDI which results in the stabilization of the Al-H bond. Even further, these compounds demonstrate surprising thermal stability during thermolysis as evidenced by TGA results discussed above. The clean volatilization demonstrated by compound $\mathbf{2}$ and compound $\mathbf{5}$ demonstrate how this 'Pr-CDI deinsertion is much more prevalent in solution as the compounds exhibit higher stability in the gas phase.

\subsubsection{Time-of-Flight Mass Spectrometry}

The complex decomposition evident in the TGA collected for compound 3 (Figure 28) prompted further exploration into the gas phase decomposition products. Time-of-flight mass spectrometry (TOF-MS) of compound $\mathbf{3}$ was performed by a lab mate, Jason Coyle, at the University of Saskatchewan under the supervision of Dr. Jens Müller. The TOF-MS was performed on a custom built apparatus consisting of three connected vacuum chambers. Compound $\mathbf{3}$ was heated in the first chamber under reduced pressure to volatilize the compound where it would be carried through to the second chamber in a stream of argon gas, aided by a pressure differential $\left(\sim 10^{-4} \mathrm{mbar}\right.$ to $\left.\sim 10^{-8} \mathrm{mbar}\right)$. Situated between the first and second chamber is a sample skimmer with a $1.5 \mathrm{~mm}$ orfice to produce the molecular beam. Within the second chamber, the molecular beam undergoes electron impact ionization. The resultant ions are accelerated perpendicular to the molecular beam, into the TOF-MS situated in the third and final vacuum chamber ( $\left.10^{-9} \mathrm{mbar}\right)$. A $4.35 \mathrm{kV}$ mass filter was used to reduce the signal of the argon carrier gas preventing the detector from exceeding its detection limit. 


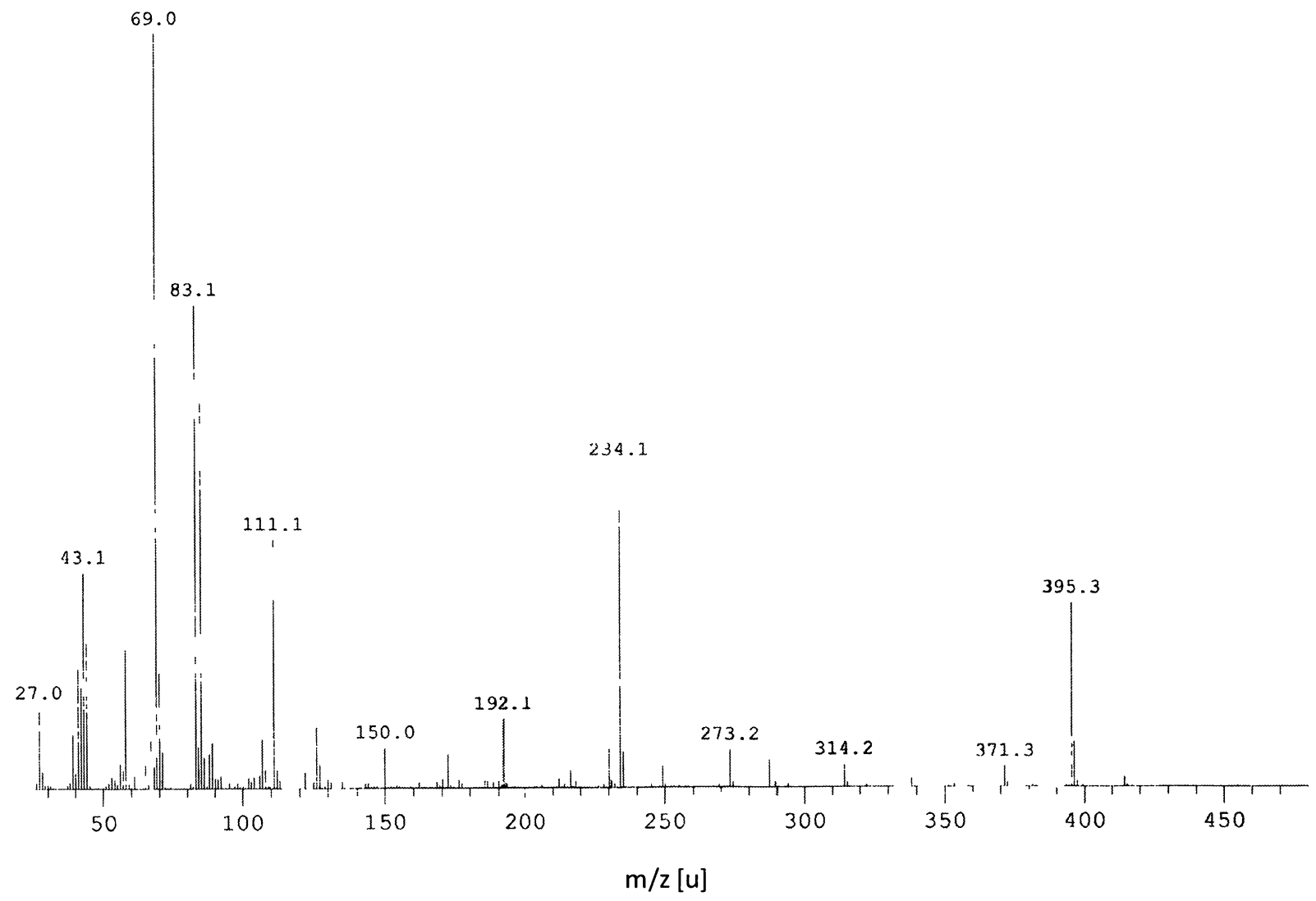

Figure 35. Electron impact mass spectrum of compound 3 at room temperature

TOF-MS experiments were performed on compound 3 at 15 different thermolysis temperatures ranging from $150^{\circ} \mathrm{C}$ to $500^{\circ} \mathrm{C}$. Figure 35 shows the electron impact MS collected for compound 3, demonstrating the fragmentation pattern at room temperature. The parent peak in the spectra at $395.3 \mathrm{u}$ represents M-1 of compound 3, which can be found as the predominant mass fragment detected during TOF-MS studies in Figure 36 . The intensity of $395.3 \mathrm{u}$ peak remains constant until about $225^{\circ} \mathrm{C}$ where the fragment intensity is found to decrease until at about $350^{\circ} \mathrm{C}$. It is at this temperature where complete loss of $395.3 \mathrm{u}$ detection occurs. Complete loss of signal for the parent compound can be attributed to complete thermal decomposition of compound 3. Throughout the set of TOF-MS experiments, six mass fragments are tracked with structures of interest shown in Figure 37. 


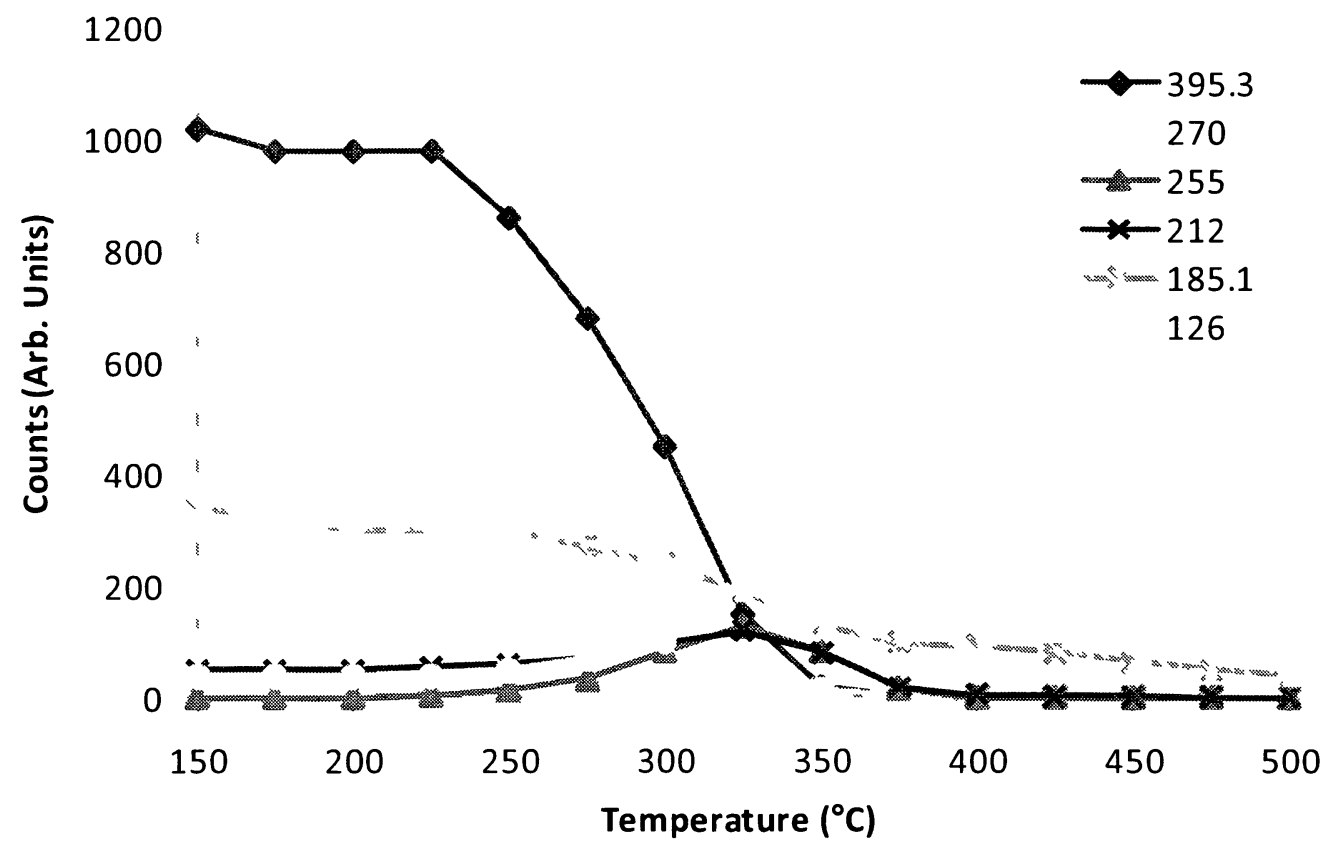

Figure 36. Temperature dependence of selected mass fragments measured by TOF-MS during thermolysis compound 3

During the fragmentation of compound 3, five other mass fragments were found to emerge from the baseline data. Even at lower temperatures, while the parent peak for compound $\mathbf{3}$ is still relatively high, a peak representing $185.1 \mathrm{u}$ is also present and decreases with increasing temperature. $185.1 \mathrm{u}$ corresponds to the mass of triisopropyl guanidine, shown in Figure 36, demonstrating the loss of a ligand from the parent compound. The presence of this peak suggests that a small amount of thermal decomposition exists even at low temperatures as the peak is not present in the fragmentation pattern collected at room temperature. This indication of thermal decomposition at low temperatures supports the decomposition found in the TGA data collected for the compound found in Figure 28. However, air-sensitivity of compound $\mathbf{3}$ often resulted in the production of triisopropyl guanidine, as discussed above. The fact that the $185.1 \mathrm{u}$ mass fragment remains at high temperatures even when the detection of compound $5 \mathrm{M}-1$ has ceased, suggests that the presence of guanidine 
may not be due to thermal decomposition, but rather from hydrolysis due to air exposure. The introduction of air to the sample may have simply occurred during transportation to the University of Saskatchewan where it had to be removed from the glovebox. Even with a parafilm seal around the vial cap, small amounts of air may have made its way into the vial. Whether or not air exposure happened during transit, the sample may also have inadvertently been exposed to air during manipulation and loading into apparatus, or simply small air leaks in the apparatus.

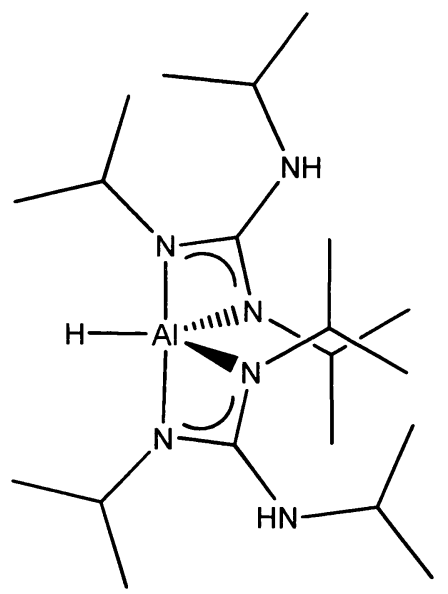

$396 u$

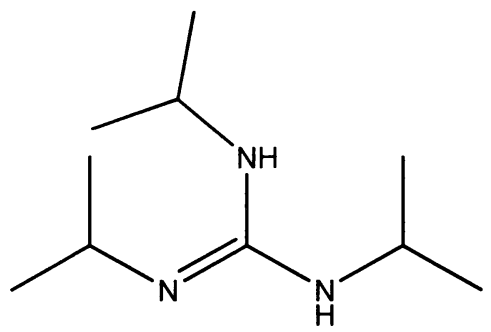

$185 \mathrm{u}$

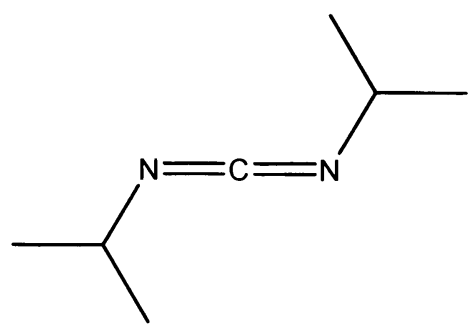

$126 u$

Figure 37. Mass fragments of interest

Interestingly, the decrease in $185.1 \mathrm{u}$ peak detection is mirrored by the increase in $126 \mathrm{u}$, a mass fragment representing iPr-CDI. The point at which these two mass fragments intersect, there is a surprising maximum found for mass fragments $270 u, 255 u$, and $212 u$. It is difficult to assign structural representations of these peaks with any certainty, but the masses $270 \mathrm{u}$ and $212 \mathrm{u}$ correspond to the deinsertion of ' $\mathrm{Pr}-\mathrm{CDI}$ and loss of a guanidine ligand from the parent compound, respectively. Thus, the fragmentation of compound 3 suggests that as the 
thermolysis passes from a loss of guanidine into a realm of 'Pr-CDI deinsertion, a balance is found in which the production of 4-coordinate aluminum species is at a maximum.

Or, in the case of the $185.1 \mathrm{u}$ peak being present due to air-exposure, then perhaps it is just coincidence that the maxima occur when the $185.1 \mathrm{u}$ and $126 \mathrm{u}$ signals cross, or the deinsertion of 'Pr-CDI from 185.1u. In this case, the thermolysis of compound $\mathbf{3}$ could be attributed completely to the deinsertion of 'Pr-CDI. This thermal decomposition would be more likely since 'Pr-CDI deinsertion is very common of amidinate compounds. $(26 ; 27 ; 47 ; 49 ; 28)$ Also supporting this hypothesis is the fact that mass fragments $270 u, 255 u$, and $212 u$ all follow the same detection pattern, suggesting that perhaps mass fragment $212 \mathrm{u}$ corresponding to the loss of guanidine from the parent compound was also coincidence, but instead, $212 \mathrm{u}$ and $255 \mathrm{u}$ are simply due to fragmentation of $270 \mathrm{u}$. It can be hypothesized that $255 \mathrm{u}$ represents the loss of a methyl group from $270 u$, and $212 \mathrm{u}$ represents the loss of an isopropyl amide group. Thus, all three mass units can be attributed to a potential 4-coordinate aluminum hydride species.

This production of a potential 4-coordinate aluminum species is quite interesting from a deposition point of view. The synthesis of 4-coordinate aluminum hydrides were unable to be performed as attempts often lead to oligomerization and literature procedures typically isolated dimers. $^{(39 ; 40)}$ If some control were able to be exercised, the thermolysis of compound 3 could lead to the gas phase production of a 4-coordinate aluminum species which could then be used to deposit aluminum metal films. If this were the case, a new method of deposition would be found, developing a process with a "pre-precursor": essentially a precursor to the deposition precursor. 


\section{ALUMINUM METAL DEPOSITION}

\subsection{Deposition Methods}

It is evident in the characterization chapter that compound $\mathbf{2}$ and compound $\mathbf{5}$ possess the best thermal characteristics and are the best candidates for use as deposition precursors. However, some of the thermolysis results were not performed until after deposition studies had commenced and compound $\mathbf{5}$ was not successfully synthesized and isolated until recently. Compound $\mathbf{3}$ was selected to perform deposition studies due to its ease of synthesis and thermal stability as determined by solution based ${ }^{1} \mathrm{H}$ NMR thermolysis studies.

While extreme air-sensitivity and the evidence of poor thermal stability can be attributed to compound $\mathbf{3}$, deposition studies were investigated. With the TOF-MS results presenting the possibility of the formation of a gas phase 4-coordinate aluminum precursor, the study of compound $\mathbf{3}$ deposition remains very interesting and worth-while investigating.

\subsubsection{Deposition Reactor}

Thin film depositions were performed using a custom made hot-walled atomic layer deposition reactor, as depicted in Figure 38. The reactor body is built around a Lindburg/Blue "Mini-Mite" tube furnace $(1 \mathrm{~cm}$ wide by $12 \mathrm{~cm}$ long) with an integrated, programmable temperature controller, providing a temperature range from $100^{\circ} \mathrm{C}$ to $1100^{\circ} \mathrm{C}$. A "Vacuum Deposition System", custom made by Angstrom Engineering Inc., provides electrical connections and pressure control to a variety of components in the system. The reactor is equipped with a valve array consisting of 5 pneumatically actuated valves purchased from Swagelok fluid system technologies. The valves are rated to a maximum temperature of $200^{\circ} \mathrm{C}$, 
and are heated externally using heating tape purchased from VWR. The heating tape is also used to heat a stainless steel precursor bubbler, made from components purchased from Swagelok, and stainless steel gas lines also obtained from Swagelok. The valve array, bubbler and lines are separated into 3 independent heating zones controlled by an Omron E5ZN-SDL modular temperature controller built into the Vacuum Deposition System. Nitrogen carrier gas flow in the system is controlled using a CCR Entela mass flow controller set to $200 \mathrm{sccm}$, or 50 sccm for low pressure experiments.

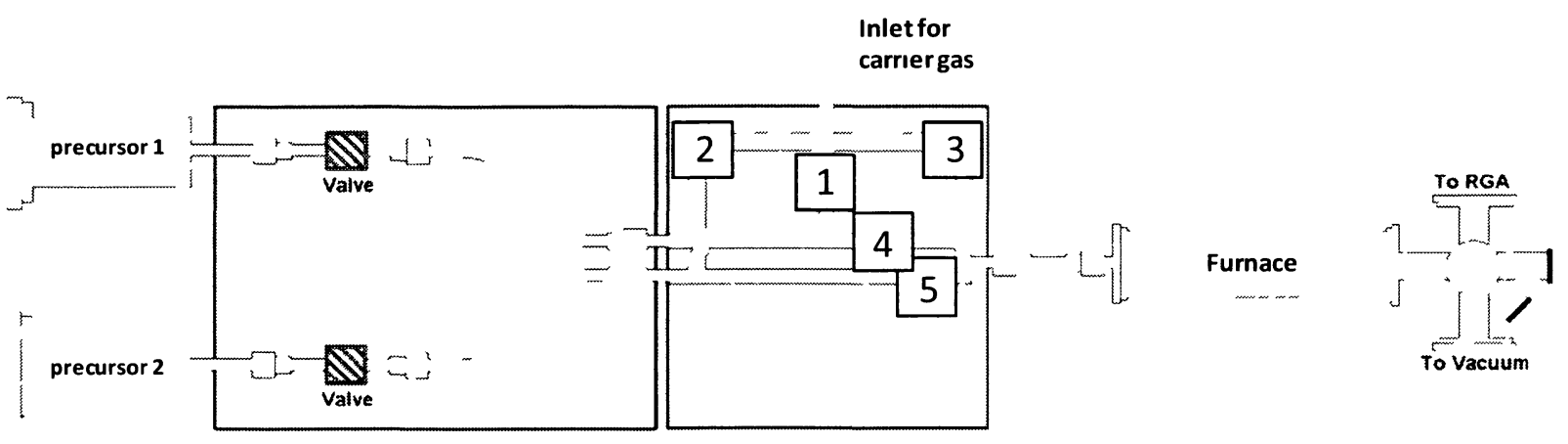

Figure 38. Schematic diagram of custom made hot-walled ALD reactor

The pneumatic valve array is controlled using a custom program written using LabVIEW software by National Instruments. The program allows for independent control of the 5 valves providing a platform for an ALD cycle to be programmed and repeated as desired. Valve 1 is used to control the flow of $\mathrm{N}_{2(\mathrm{~g})}$ carrier gas into the first precursor bubbler. Valve 2 provides the same service as valve 1 only to introduce a carrier gas to bubbler 2 . Since the secondary precursor in this work is forming gas $\left(5 \% \mathrm{H}_{2(\mathrm{~g})}\right.$ in $\left.\mathrm{N}_{2(\mathrm{~g})}\right)$, then valve 2 is not required. Valve 3 provides a direct line of $\mathrm{N}_{2(\mathrm{~g})}$ carrier gas to the reactor furnace. Valve 4 provides direct flow from bubbler 1 into the furnace. Similarly, valve 5 provides direct flow from bubbler 2 through to the furnace. 
The detailed experimental for substrate preparation as well as pulsed CVD experiments can be found in sections 5.2.1 and 5.2.2. Parameters used for each deposition experiment can be found in Appendix C with further characterization.

\subsubsection{Pulsed CVD}

The pulse program used for the deposition was modelled after one that was used previously. $(25,41)$ Figure $39 \mathrm{~A})$ presents the pulse program used in the first 5 preliminary deposition experiments. As thicker films are easier to characterize, attempts at growing thicker films by increasing the number cycles was attempted and found to be unsuccessful. Even increasing the number of cycles to over 800 was found to deposit only trace amounts of aluminum metal, detectable by $\mathrm{PXRD}$. Increasing the furnace temperature to promote nucleation at the substrate surface also failed to produce a thicker film. It was instead discovered that the pulse program was the issue causing the insufficient introduction of precursor to the reactor furnace.

A)

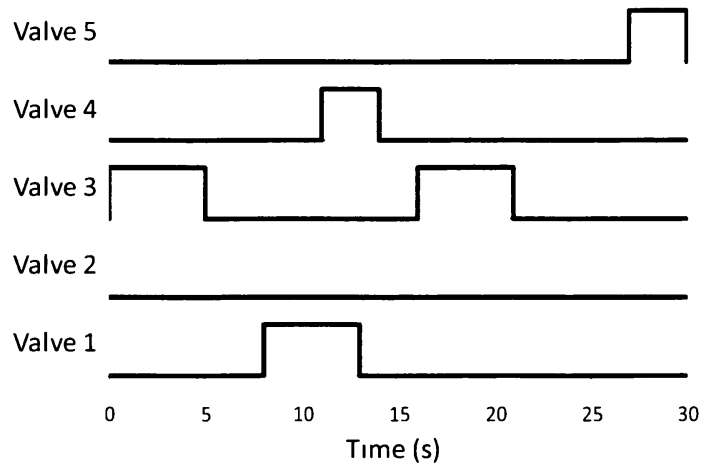

B)

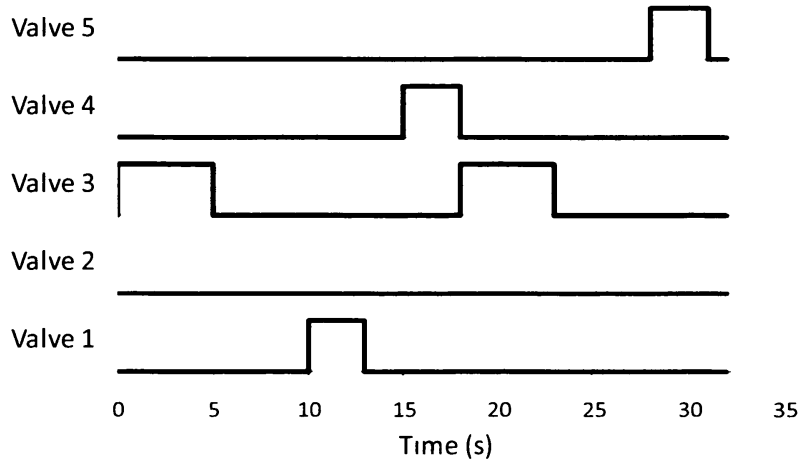

Figure 39. Pulse program used for A) preliminary experiments and B) bulk of deposition experiments

As shown in Figure 39A), an overlap in valve 1 and valve 4 exists. Valve 1 is opened first to bring nitrogen gas into the bubbler to mix with the precursor so it may carry the precursor to 
the reaction furnace upon opening valve 4 . It is thought that having valve 1 and 4 open at the same time would cause the carrier gas to bypass the bubbler altogether and pass through the lines causing the precursor vapour to be suppressed and remain trapped in the bubbler. The fact that any aluminum metal deposition occurred at all with this pulse program was quite lucky as small amounts of the precursor must have been able to make its way into the stream of flowing nitrogen. Since this discovery, the pulse program was fixed to that shown in Figure 39B) where valve 1 is closed after bringing nitrogen into the precursor bubbler and allowed to linger within the bubbler for a couple seconds to ensure mixing of the precursor vapour and the carrier gas. Valve 4 is then opened to allow the precursor to be carried through to the reaction chamber. This change in pulse program allowed for the deposition of thicker films which were able to be imaged using the SEM, allowing the pXRD data collected to be corroborated.

Using the sublimation temperature as a guide, the bubbler temperature of compound 3 was selected. Since the sublimation of compound 3 was performed at a pressure of $105 \mathrm{mTorr}$, and the ALD reactor was determined to have a base pressure in the range of approximately 70 $90 \mathrm{mTorr}$, the bubbler temperature was first set to $15^{\circ} \mathrm{C}$ below the $95^{\circ} \mathrm{C}$ sublimation temperature. A series of deposition experiments were performed varying the temperature of the bubbler. No film deposition was observed when the bubbler was set to $80^{\circ} \mathrm{C}$, the temperature was increased and successful aluminum metal deposition was found to occur at $95^{\circ} \mathrm{C}$. A bubbler temperature of $95^{\circ} \mathrm{C}$ was found to be sufficient to promote the volatilization of compound 3 and was used for the remainder of the deposition experiments. 
The furnace temperature was selected in a similar manner. Based on previous ALD processes, the initial furnace temperature was selected to be $250^{\circ} \mathrm{C}$, which fortunately resulted in aluminum metal deposition. The furnace temperature was increased up to $350^{\circ} \mathrm{C}$ in an attempt to promote further nucleation at the substrate surface.

\subsection{Film Characterization}

Characterization of the deposited films was primarily performed using powder X-ray diffraction ( $p X R D$ ) and scanning electron microscopy (SEM). Further analysis of films using SEM and energy dispersive X-ray spectroscopy (EDS) were attempted and will be discussed. The deposited films were reflective and coloured similarly to the silicon wafer substrates making it very difficult to determine whether a film deposition was successful or not. For this reason, the use of characterization methods was essential in monitoring the progress as deposition parameters were modified.

\subsubsection{Powder XRD}

Powder X-ray diffraction ( $\mathrm{pXRD)}$ is a technique which provides crystallographic information using Bragg's law. X-rays are diffracted off of the surface of a crystal and the intensity of X-rays being diffracted are detected. By varying the angle of detection, the intensity of X-rays changes depending on the crystallinity of the surface. Figure 40 demonstrates the powder pattern collected for a film deposited by compound $\mathbf{3}$ using the pulse CVD method described above. Bragg's law (eq. 4) can be used to calculate the lattice spacing of a given cubic system using Miller indices of the Bragg plane (eq.5). 


\section{Bragg's Law:}

$2 d \sin \theta=n \lambda$

Miller Indices of the Bragg Plane:

$d=\frac{\mathrm{a}}{\sqrt{h^{2}+k^{2}+l^{2}}}$

$\left(\frac{\lambda}{2 a}\right)^{2}=\frac{\sin ^{2} \theta}{h^{2}+k^{2}+l^{2}}$

*Please note, relation in Eq 5 is only applicable to cubıc crystal systems

Compositional information can also be gained as powder pattern data can often be compared to databases of characterized materials. Using an online crystallographic database, a search of the peaks resultant in the powder pattern collected (shown in Figure 40) was performed. ${ }^{(53)}$ The database retrieved literature sources which demonstrated this diffraction pattern, naming the material analyzed as aluminum metal. ${ }^{(54)}$

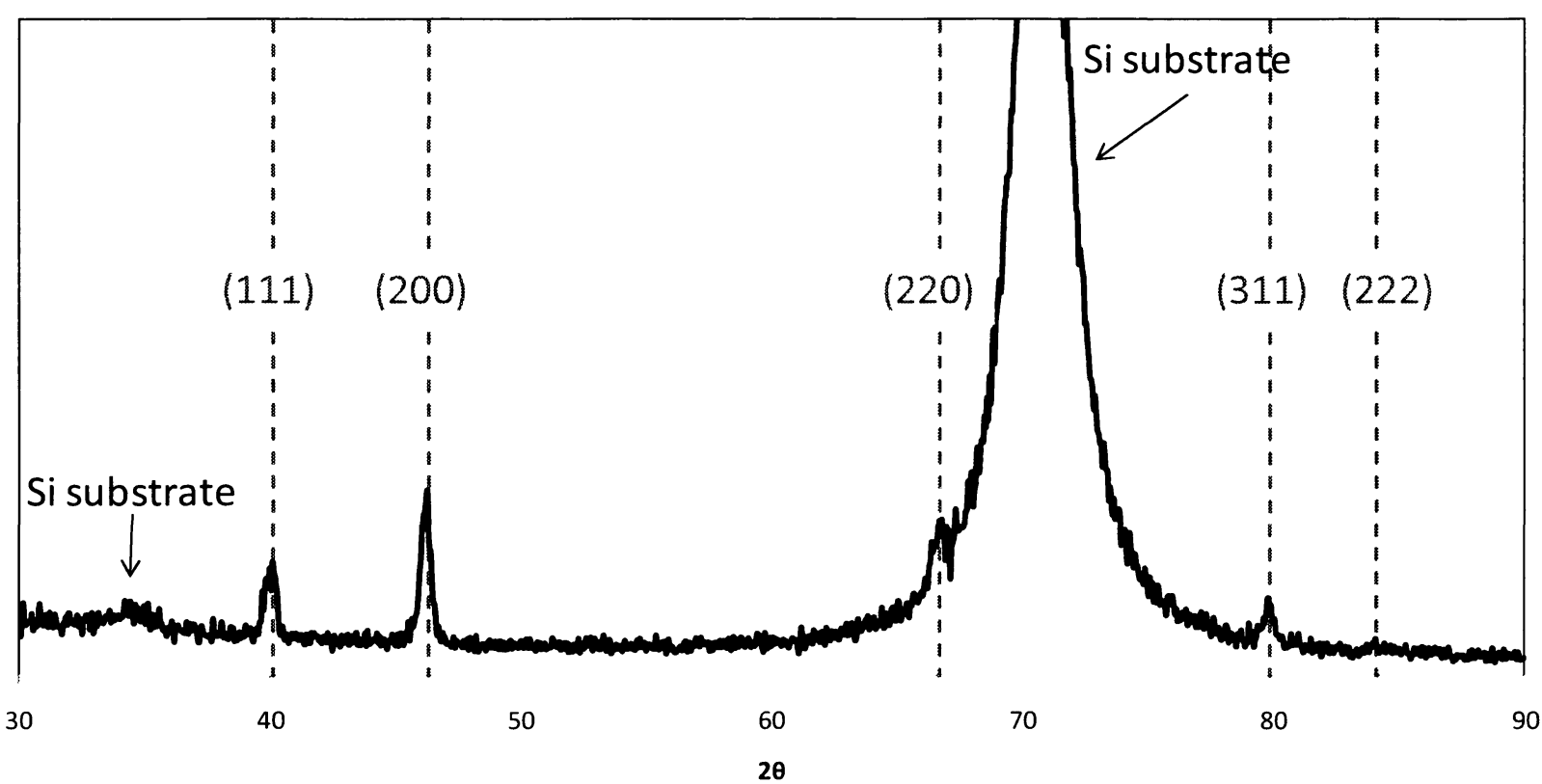

Figure 40. XRD powder pattern of film deposited with compound 3, overlaid with literature powder pattern for ccp aluminum metal ${ }^{(54) \neq}$ 
The literature powder pattern is shown in Figure 40 as vertical dotted lines which help demonstrate how well the deposited film matches the literature material. Table 9 reiterates this precision as only one of the peaks in the powder pattern is off by $0.1^{\circ}$. The Miller indices listed in Table 10 are that which were reported in the literature source.

Table 9. Comparison of experimental and literature PXRD data ${ }^{(54) \neq}$

\begin{tabular}{ccc}
\hline Experimental Peak & Literature Peak & Miller Indices \\
\hline $38.5^{\circ}$ & $38.5^{\circ}$ & $\left(\begin{array}{lll}1 & 1 & 1\end{array}\right)$ \\
$44.7^{\circ}$ & $44.8^{\circ}$ & $\left(\begin{array}{lll}2 & 0 & 0\end{array}\right)$ \\
$65.2^{\circ}$ & $65.2^{\circ}$ & $\left(\begin{array}{lll}2 & 2 & 0\end{array}\right)$ \\
$78.3^{\circ}$ & $78.3^{\circ}$ & $\left(\begin{array}{lll}3 & 1 & 1\end{array}\right)$ \\
$82.5^{\circ}$ & $82.5^{\circ}$ & $\left(\begin{array}{lll}2 & 2 & 2\end{array}\right)$ \\
\hline
\end{tabular}

The deposited films are confirmed as being aluminum metal, the Miller indices allow the determination of the space group as $\mathrm{Fm} \overline{3} \mathrm{~m}$, and the crystal packing as cubic closest packed (ccp). The other two peaks evident in Figure $40,36^{\circ}$ and $71^{\circ}$, can be attributed to the silicon wafer. The intensity of these silicon peaks suggests poor film coverage or penetration of the $X$ rays through a thin aluminum film to also characterise the underlying substrate. As will be shown using SEM, film coverage appears to be complete for most deposited films.

Although pXRD is a powerful tool in detecting the presence of aluminum metal, it does have one down fall: since the method operates on the detection of diffracted X-rays, amorphous materials often go undetected. Amorphous materials tend to scatter the X-rays resulting in very broad peaks which can get lost in the baseline. Thus, even though the method proves the presence of aluminum metal, it cannot confirm the absence of amorphous aluminum oxide which may result from small air leaks in the system as well as a surface passivation layer. 


\subsubsection{Scanning Electron Microscopy}

Scanning electron microscopy (SEM) of the deposited films were collected on a Tescan Vega-II XMU VPSEM most often operating at 30kV high vacuum mode providing a theoretical minimum resolution of $3 \mathrm{~nm}$. SEM employs an electron beam to raster scan the sample providing secondary electrons for detection. Through this method, information regarding the deposited films has been gained. Once the material has been identified as aluminum metal by pXRD, SEM methods are used to understand and tune the deposition process.

Figure 41 demonstrates how SEM can be used to help tune a deposition process. The snowflake formations found in waves on the surface is typical of crystalline precursor or ligand by-products which have nucleated on the surface and/or become trapped in the depositing film. These formations suggest insufficient mass flow within the reactor caused by a low flow of nitrogen purge gas. Without proper purge gas flow, the by-products of surface reaction are unable to be completely removed from the reaction chamber between precursor pulses. The flow rate and purge times for the deposition experiment had not been changed from other depositions which did not present these compound formations, rather, the nitrogen cylinder simply ran out of pressure over the course of the experiment resulting in low purge gas flow at the tail end of the deposition.

Once deposition flow rates, temperature zones, and pulse program were all optimized, the resultant films were smooth and often featureless. SEM became difficult to perform as there were no definable features to use to focus the instrument. Thus, the introduction of defects in the surface was used to verify the presence of the film, as shown in Figures 42-43. 


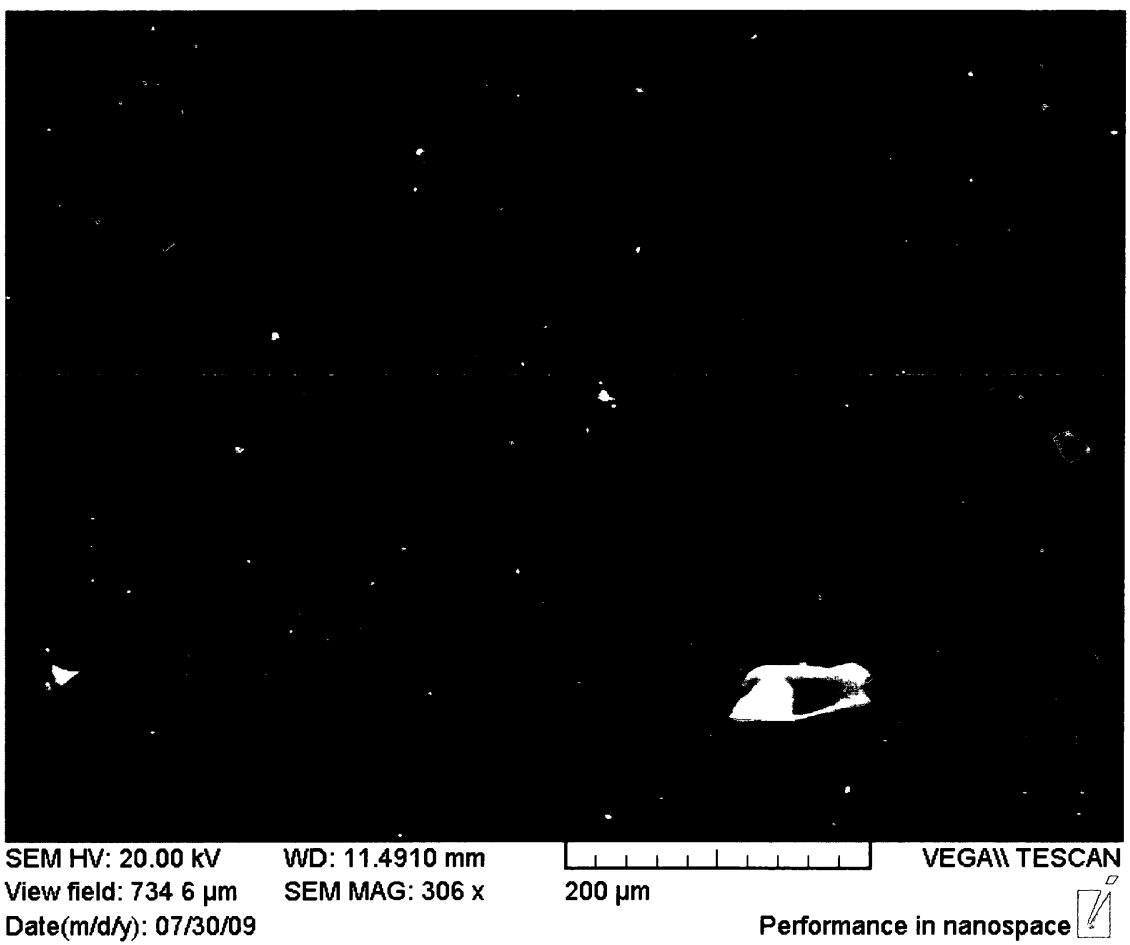

Figure 41. Compound trapped within film

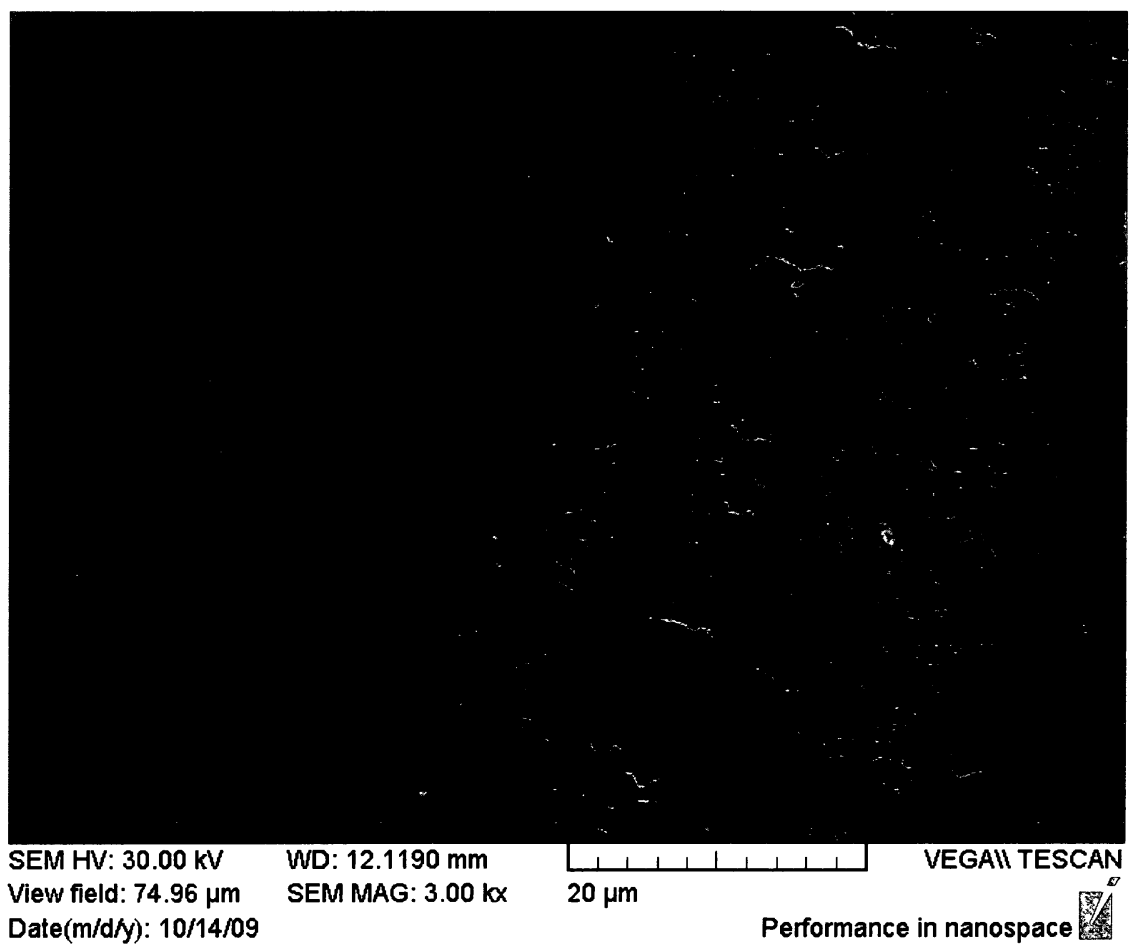

Figure 42. Scratch in film surface 
Figure 42 demonstrates a scratch test in which a sharp edge was dragged through the film. The track made by the razor can provide some insight into the films composition. With pXRD, it was confirmed that the film possessed aluminum metal in a ccp structure. However, the pXRD method falls short as the presence of amorphous materials often goes undetected. The flaking demonstrates a hard brittle film, supporting that the film is continuous aluminum metal as opposed to crystalline aluminum metal in a matrix of aluminum oxide.

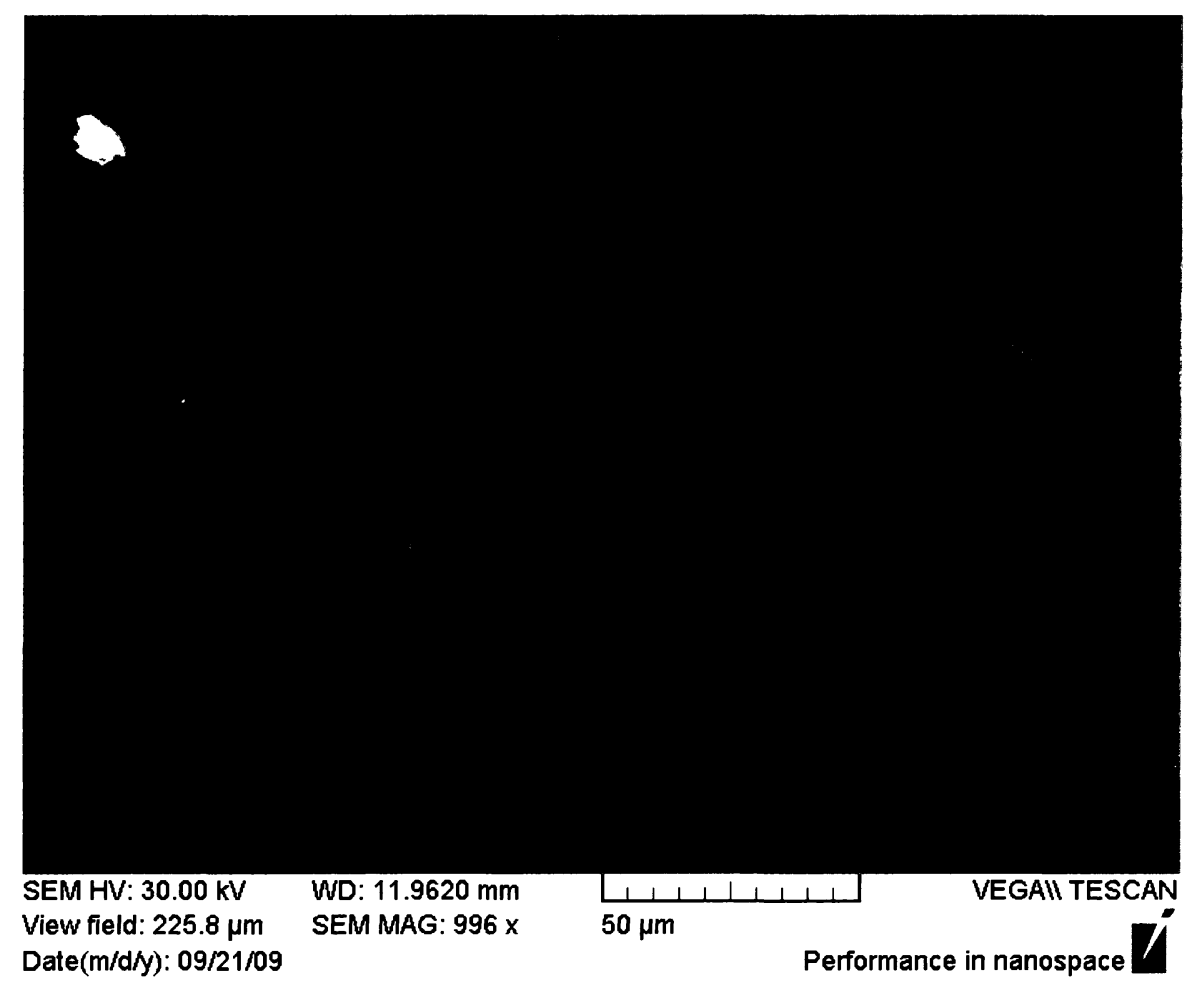

Figure 43. Scotch tape test of aluminum metal film

Figure 43 shows the result of a Scotch tape test. The scotch tape test is a simple method for evaluating the adhesion of the deposited film to the surface of the substrate. A piece of Scotch brand tape was applied to half of the deposited film and pressed down firmly using the back of a finger nail. After a resting period of about 1 minute, the tape was quickly removed in one fluid motion. Films which exhibit poor adhesion often become removed during this process 
as the film preferentially adheres to the tape as opposed to the substrate. For the deposited aluminum metal films, there was no observable film removed on the tape. With the use of SEM, it was found that a small amount of lifting did occur along the edge where the tape was adhered. Figure 43 shows an area of the film such that the tape was position in the foreground along the edge of the crack. Small pinhole formations on the lower edge indicate areas where some film was pulled off. As can be seen in the top left hand corner of the SEM, a piece of dust that may have fallen on the surface inside the reactor remains on the surface, any such formations on the area which was pressed with tape would be removed. The smooth, continuous nature of the film remains intact for the most part indicating a fairly adequate adhesion of the film to the substrate surface.

\subsubsection{Difficulties In Film Characterization}

Energy dispersive X-ray spectrometry (EDS) was attempted for elemental analysis of the deposited films. The electron beam within the SEM is focused on the sample where ground state electrons are excited and ejected from the electron shell. Higher energy electrons then drop down in energy in order to fill the hole that is left behind. As the electrons drop down in energy levels, they release energy in the form of radiation (X-rays). These detected $\mathrm{x}$-rays provide elemental analysis, as the energy difference between states is unique for each element.

Figure 44 represents a characteristic EDS collected for a typical deposited aluminum metal sample (same sample as shown in Figure 42). The detection of aluminum metal by this method is complicated by the fact that aluminum and silicon have similar discrete energies $1.4 \mathrm{keV}^{(55)}$ and $1.6 \mathrm{keV},{ }^{(56)}$ respectively. A difference of $0.2 \mathrm{keV}$ should provide enough difference between the two, but as apparent in Figure 44, a significant overlap exists between $\mathrm{Al}$ and $\mathrm{Si}$ 
making it difficult to distinguish the film from the substrate. The baseline of the EDS spectrum is also quite interesting showing a detection of X-rays at a very broad range of energies.

A strong signal at about 3.4keV (labelled as "Sn") and smaller peak at about 5.2keV (labelled as "Nd") are quite suspicious. The presence of neodymium within the film or even the SEM chamber seems quite ludicrous. Furthermore, the regular spacing between the peaks seems suspect: the peaks are each separated by about $1.7 \mathrm{keV}$. Thus, the EDS results collected were unable to adequately characterize the elemental composition of the film.

In order to estimate the thickness of the deposited films, SEM was employed. For this purpose, an extra thick film was prepared, which are typically easier to characterize by this method. A 22 hour long deposition was performed consisting of 2258 cycles with a long 6 second precursor pulse. Using a diamond tipped pen, the film and substrate were scored and then broken in half. The slide was then position on a perpendicular stage mount so that SEM could be performed on the edge of the slide. As demonstrated in Figure 45, the measurement of the film thickness remained challenging: SEMs A-D represent four of many SEM images taken for a single sample, varying only by stage angle, magnification, and wavelength dispersion (WD). 


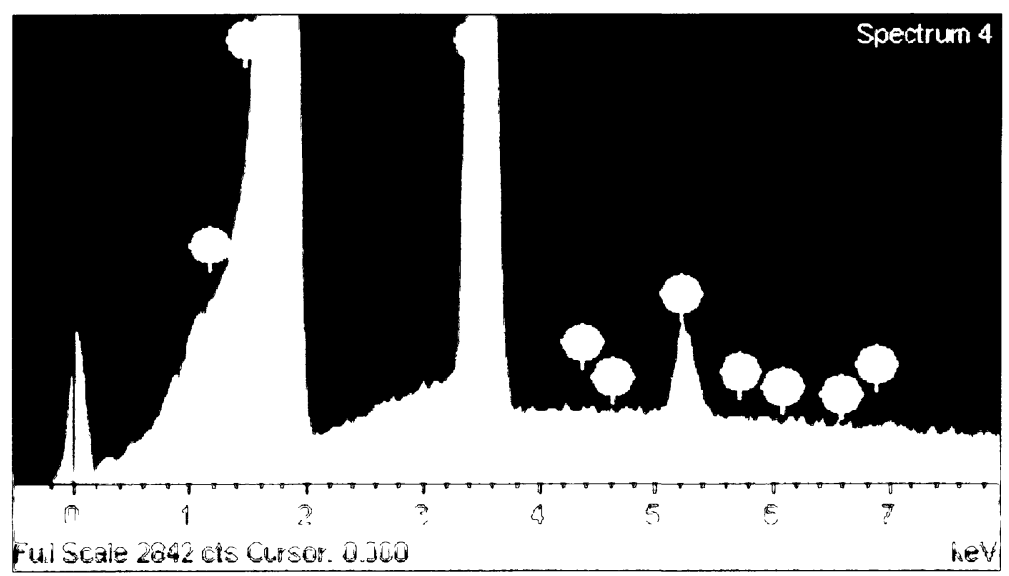

Figure 44. Energy dispersive spectroscopy of aluminum metal film

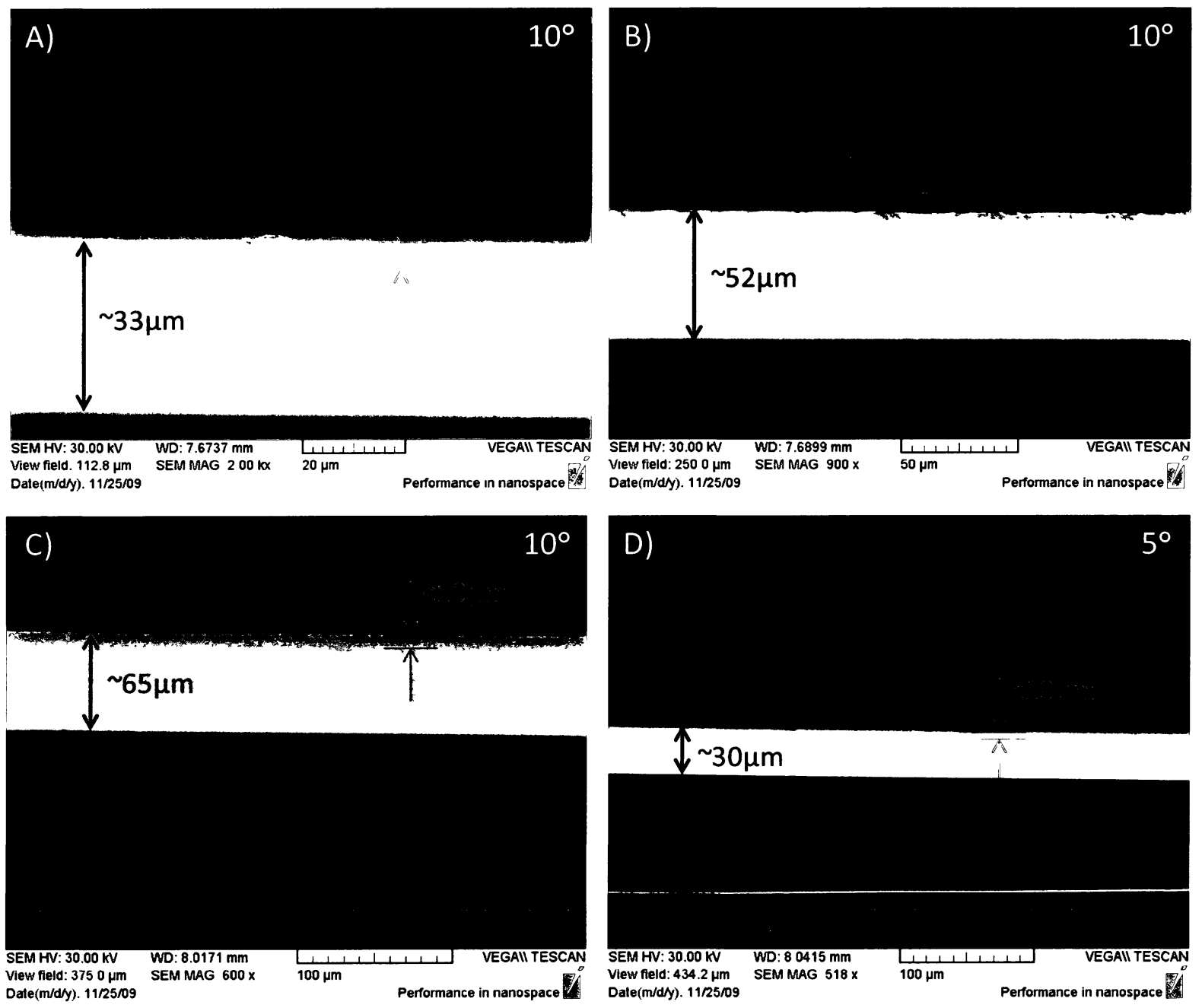

Figure 45. Edge of deposited film on Si wafer with A) $10^{\circ}$ tilt, 2000x, B) $10^{\circ}$ tilt, $900 x$, C) $10^{\circ}$ tilt, 600x, and D) $5^{\circ}$ tilt, 518x 
As was found when trying to perform elemental analysis using EDS, the discrete energies of aluminum metal and the silicon substrate are very similar. This issue also presents during imaging as the film and substrate are most often indistinguishable, requiring a high level of contrast enhancement. In images A, B, and C, an angle of $10^{\circ}$ is constant and yet the perceived film thickness ranges from approximately $33 \mu \mathrm{m}$ to $65 \mu \mathrm{m}$. A difference of about $20 \mu \mathrm{m}$ is observed between $A$ and $B$, and can be attributed to a combination of WD and magnification. Both images were collected with a WD of about $7.7 \mathrm{~mm}$ leaving magnification to be the only difference between the two. As magnification is increased, the WD must be tuned in order to properly focus the electron beam. It is possible that this focusing could be the source of the thickness variance. Furthermore, the thickness range of this perceived film relates to a deposition rate of $\sim 14.6 \mathrm{~nm}-28.8 \mathrm{~nm}$ per cycle. This deposition rate is not unheard of for a CVD process, but since this process is pulsed, the rate seems suspicious. ALD processes have growth rates on the order of $\AA$ /cycle. The 2258 cycle pulsed deposition would be expected to result in a film thickness of anywhere between say $500 \mathrm{~nm}$ and $5 \mu \mathrm{m}$.

It is instead hypothesized that the SEM images shown in Figure 45 does not actually represent the edge of the film and is instead showing a ridge created when cleaving the silicon wafer. When taking a closer look at these SEM images, a slightly darker area can be seen at the edge of the film. In Figure 45A), C), and D), This film thickness can be estimated to be around $6 \mu \mathrm{m}$ but is difficult to distinguish due to poor resolution. A $6 \mu \mathrm{m}$ film is a much more believable film thickness for the deposition resulting in a growth rate of approximately $2.7 \mathrm{~nm} / \mathrm{cycle}$. However, it is clear from this film characterization that further film analysis is required in order to continue the development of the aluminum metal deposition process. 


\section{CONCLUSIONS}

\subsection{Synthesis and Characterization of Heteroleptic Aluminum Compounds}

The synthesis of 5-coordinate heteroleptic amidinate aluminum hydride compounds were synthesized from hydrogen elimination and ligand exchange methods. Clear, colourless crystals of compounds $\mathbf{2}, \mathbf{3}$, and $\mathbf{4}$ were isolated from hydrogen elimination methods by crystallization with yields of $86 \%, 93 \%$, and $78 \%$, respectively. Clear, colourless crystals of compounds $\mathbf{1}$ and $\mathbf{5}$ were isolated from ligand exchange methods by crystallization with yields of $85 \%$ and $16 \%$, respectively.

Due to the weak aluminum hydride bonding, the synthesis of compounds 1-5 were required to be performed with care in order to avoid decomposition. The rate of the exothermic hydrogen elimination reactions was controlled by slow addition of amidine solution to a cooled alane solution to avoid thermal decomposition during synthesis. Ligand exchange provided compound $\mathbf{1}$ in good yield, but was found to be unsuitable for compound $\mathbf{5}$ which had a maximum yield of $16 \%$. The reaction was found to have a slow decomposition at room temperature, and when cooled to prevent decomposition, no reaction would proceed.

Structural characterization using SCXRD of compounds $\mathbf{1}, \mathbf{2}, \mathbf{4}$, and 5 demonstrated how slight variations in the exocyclic moiety provided trends in $\mathrm{Al}-\mathrm{H}$ bond length and amidinate bite angle relating to steric bulk and electronic character of the exocyclic position. The respective bite angles for compounds $1,2,4$, and 5 were determined to be $67.8^{\circ}, 67.5^{\circ} / 67.4^{\circ}, 67.4^{\circ} / 67.1^{\circ}$, and $66.7^{\circ}$, with respective Al-H bond lengths $1.58 \AA \AA$, $1.53 \AA \AA, 1.51 \AA \AA$, and $1.94 \AA ̊$. The chemical shift 
of the aluminum hydrides were found to be far downfield suggesting a high amount of electronic deshielding. The hydride peaks also exhibited broadening due to coupling with ${ }^{27} \mathrm{Al}$.

TGA was performed on the five compounds providing onset of volatilization $174.4^{\circ} \mathrm{C}$, $143.3^{\circ} \mathrm{C}, 153.5^{\circ} \mathrm{C}, 148.9^{\circ} \mathrm{C}$, and $118.8^{\circ} \mathrm{C}$, and residual mass $3.36 \%, 0.97 \%, 12.6 \%, 0.76 \%$, and $1.01 \%$ of compounds $1-5$, respectively. Compound 3 was found to possess a high level of decomposition upon thermolysis which was attributed to the presence of a proton in the exocyclic position. Compounds $\mathbf{2}$ and $\mathbf{5}$ were found to have a clean or controllable thermolysis and were determined to be good candidates as ALD precursors.

${ }^{1} \mathrm{H}$ NMR thermolysis studies investigated the solution decomposition processes for compounds 1-5 providing first order rate constants $4.4 \times 10^{-6} \mathrm{~s}^{-1}, 1.07 \times 10^{-6} \mathrm{~s}^{-1}, 1.49 \times 10^{-6} \mathrm{~s}^{-1}, 1.66$ $\times 10^{-6} \mathrm{~s}^{-1}$, and $4.0 \times 10^{-8} \mathrm{~s}^{-1}$, with half-lifes 1.8 days, 7.5 days, 5.4 days, 4.8 days, and 201 days for compounds 1 and 2 at $56^{\circ} \mathrm{C}$, and compounds $3-5$ at $65^{\circ} \mathrm{C}$, respectively. Decomposition of compound 1 produced four compounds which were identified as $\left[\mathrm{Me}_{2} \mathrm{NC}\left(\mathrm{N}^{\prime} \mathrm{Pr}\right)_{2}\right] \mathrm{Al}\left(\mathrm{NMe}_{2}\right)_{2}$, $\left[\mathrm{HC}\left(\mathrm{N}^{\prime} \mathrm{Pr}\right)_{2}\right]_{2} \mathrm{AlH},\left[\mathrm{HC}\left(\mathrm{N}^{\prime} \mathrm{Pr}\right)_{2}\right]_{3} \mathrm{Al}$, and $\left[\mathrm{Me}_{2} \mathrm{NC}\left(\mathrm{N}^{\prime} \mathrm{Pr}\right)_{2}\right]_{2} \mathrm{Al}\left(\mathrm{NMe}_{2}\right)$. A solution decomposition mechanism was determined to resemble the known 'Pr-CDI deinsertion mechanism with an additional reinsertion to stabilize the $\mathrm{Al}-\mathrm{H}$ bond and with ligand exchange processes.

TOF-MS studies were employed to further investigate the gas phase thermolysis of compound 3. Mass fragments detected during the gas phase thermolysis demonstrate an increase in 'Pr-CDI production as well as the presence of a potential 4-coordinate compound. This thermolysis suggests the possible gas phase evolution of a 4-coordinate precursor which may be the source of deposition during pulsed CVD experiments. 


\subsection{Aluminum Metal Deposition and Film Characterization}

Thin films of aluminum metal were grown using a pulsed CVD method using a hotwalled ALD reactor. The films were analyzed by $\mathrm{PXRD}$ and found to be aluminum metal in a cubic closest packed (cсp) arrangement. The film was found to be smooth and demonstrated fairly good adhesion, as demonstrated by a scotch tape test. The pulse sequence, temperature and gas flow within the reactor were optimized. Further optimization regarding growth rate was unable to be performed as accurate thickness measurements were unable to be achieved.

\subsection{Future Directions}

The results contained within this thesis exhibit great progress, and promise, in the development of a deposition process for aluminum metal thin films. However, before such a process can be used practically, for the deposition of interconnect materials there is much more research to be performed. The mechanism of deposition must be investigated further: is the successful deposition of aluminum metal films due to the gas phase decomposition of compound $\mathbf{3}$ into a 4-coordinate aluminum precursor, treating compound $\mathbf{3}$ as a "preprecursor"? Does compound 5, which seems to have superior thermal characteristics among the group, also produce aluminum metal thin films using only forming gas as a reducing agent? Would these depositions occur through the same mechanism, or would the strong C-C bond between the quaternary carbon and the exocyclic methyl group in compound $\mathbf{5}$ prevent ${ }^{\mathrm{P}} \mathrm{Pr}-\mathrm{CDI}$ deinsertion allowing for a more controlled deposition?

The characteristics of compound $\mathbf{5}$ appear to be quite promising and deserve further investigation. However, the current method of synthesis provides a very low yield as 
decomposition occurs in solution at room temperature, and at lower temperature synthesis does not proceed. Attempts at synthesizing acetamidine to use for a hydrogen elimination synthetic method were attempted but unsuccessful. Hydrogen elimination remains a promising method of synthesis for compound $\mathbf{5}$ and given more time for this project, the method would be revisited as efforts were not exhausted.

The deposition of aluminum metal will remain un-optimised until a dependable analysis method can be found in order to determine the thickness of the film. Analysis methods known to our group were all attempted and found to be unsuccessful. Other techniques used in the field require specialized equipment which our lab does not possess and are costly to have performed by another party. Once a method is found, then a series of films need to be grown with varying precursor pulse lengths in order to determine if these compounds exhibit selflimiting behaviour and if an ALD process could be possible. 


\section{EXPERIMENTAL}

\subsection{Heteroleptic Aluminum Hydrides}

\subsubsection{General Procedures}

All manipulations were performed in an MBraun Unilab nitrogen filled glove box unless otherwise indicated. Alane $\mathrm{N}, \mathrm{N}$-dimethylethylamine complex solution $(0.5 \mathrm{M}$ in toluene), aluminum chloride (reagent grade, 98\%), trimethyl aluminum (2.0M in hexanes), butyl lithium (2.5M in hexanes), methyl lithium (1.6M in diethyl ether), lithium dimethylamide (95\%), $N, N^{\prime}-$ diisopropylcarbodiimide (99\%), diethylamine ( $\geq 99.5 \%)$, isopropylamine $(\geq 99.5 \%)$, and diisopropylamine $(\geq 99.5 \%)$ were purchased from Aldrich Chemical Co. and used as received. Reagent grade, anhydrous solvents; toluene (99.5\%), ethyl ether (99\%), and hexanes (99\%), were purchased from Caledon Laboratories Ltd. These solvents were stored in an MBraun solvent purification system (MB-SPS) and then transferred into a glove box and stored over molecular sieves $(3 \mathrm{~A}, 3.2 \mathrm{~mm})$ prior to use. $\left[\mathrm{Me}_{2} \mathrm{NC}\left(\mathrm{N}^{\prime} \mathrm{Pr}\right)_{2}\right]_{3} \mathrm{Al}{ }^{(47)},\left[\mathrm{MeC}\left(\mathrm{N}^{\prime} \mathrm{Pr}\right)_{2}\right]_{3} \mathrm{Al}{ }^{(49)}, \mathrm{Al}_{2}\left(\mathrm{NR}_{2}\right)_{6}$ (57) , lithiated diethyldiisopropyl guanidinate ${ }^{(43)}$, lithiated tetraisopropyl guanidinate ${ }^{(43)}$, and triisopropyl guanidine ${ }^{(58)}$ were prepared following the literature procedures indicated.

All NMR spectra were collected on a Bruker AMX 300 spectrometer using deuterated benzene as a reference. Melting point determination was performed on an Electrothermal capillary melting point apparatus made by Mel-Temp. Electron impact mass spectrometry was performed by the mass spectrometry centre at the University of Ottawa. Single crystal X-ray diffraction was solved by Dr. Glenn Yap at the University of Delaware. Thermogravimetric analysis was performed on a TGA Q500 by TA instruments. 


\subsection{2 $\left[\mathrm{Me}_{2} \mathrm{NC}\left(\mathrm{N}^{\mathrm{i}} \mathrm{Pr}\right)_{2}\right]_{2} \mathrm{AlH}(1)$}

In a $50 \mathrm{~mL}$ pressure vessel, $\left[\mathrm{Me}_{2} \mathrm{NC}\left(\mathrm{N}^{\prime} \mathrm{Pr}\right)_{2}\right]_{3} \mathrm{Al}(1.050 \mathrm{~g}, 1.95 \mathrm{mmol})$ was dissolved in toluene $(30 \mathrm{~mL})$ with stirring. A solution of alane complexed with $\mathrm{N}, \mathrm{N}$-dimethylethylamine (3.0mL, $0.5 \mathrm{M}$ in toluene, $1.5 \mathrm{mmol}$ ) was added dropwise, in excess to $\left[\mathrm{Me}_{2} \mathrm{NC}\left(\mathrm{N}^{\prime} \mathrm{Pr}\right)_{2}\right]_{3} \mathrm{Al}$ and the pressure vessel was heated to $50^{\circ} \mathrm{C}$ for 24 hours. The solution was concentrated by vacuum and left to crystallize at $-30^{\circ} \mathrm{C}$. Clear colourless crystals of compound $\mathbf{1}$ were collected by filtration (0.916g, 2.5mmol, 85\%). M.P.: $61-64^{\circ} \mathrm{C} .{ }^{1} \mathrm{H}$ NMR $\left(300 \mathrm{MHz}, \mathrm{C}_{6} \mathrm{D}_{6}\right): 4.25$ [broad, $1 \mathrm{H}, \mathrm{Al}-\mathrm{H}$ ], 3.54 [sept, $\left.4 \mathrm{H}, \mathrm{CH}\left(\mathrm{CH}_{3}\right)_{2}\right], 2.48\left[\mathrm{~s}, 12 \mathrm{H}, \mathrm{N}\left(\mathrm{CH}_{3}\right)_{2}\right], 1.40\left[\mathrm{~d}, 24 \mathrm{H}, \mathrm{CH}\left(\mathrm{CH}_{3}\right)_{2}\right] .{ }^{13} \mathrm{C} \mathrm{NMR}\left(300 \mathrm{MHz}, \mathrm{C}_{6} \mathrm{D}_{6}\right)$ : $169.47\left[\mathrm{NC}\left(\mathrm{N}\left(\mathrm{CH}_{3}\right)_{2}\right) \mathrm{N}\right], 46.14\left[\mathrm{CH}\left(\mathrm{CH}_{3}\right)_{2}\right], 39.27\left[\mathrm{~N}\left(\mathrm{CH}_{3}\right)_{2}\right], 25.06\left[\mathrm{CH}\left(\mathrm{CH}_{3}\right)_{2}\right] . \mathrm{MS}: \mathrm{m} / \mathrm{z}(\%)$ : $367.3(2.7)[M-1]$.

\subsection{3 $\left[\mathrm{Et}_{2} \mathrm{NC}\left(\mathrm{N}^{\mathrm{P} P r}\right)_{2}\right]_{2} \mathrm{AlH}(2)$}

Diethylamine $(1.90 \mathrm{~g}, 26 \mathrm{mmol})$ was diluted with diethyl ether $(40 \mathrm{~mL})$ and placed in a freezer $\left(-30^{\circ} \mathrm{C}\right)$ to cool. To this solution, butyl lithium $(10 \mathrm{~mL}, 2.5 \mathrm{M}$ in hexanes, $25 \mathrm{mmol})$ was added dropwise slowly with stirring. After 1.5 hours, a solution of $N, N^{\prime}$-diisopropylcarbodiimide $(3.16 \mathrm{~g}, 25 \mathrm{mmol})$ diluted with diethyl ether $(15 \mathrm{~mL})$ was added dropwise to the reaction flask. The solution was left stirring overnight to form lithiated diethylguanidinate. The guanidinate was removed from the nitrogen filled glove box and hydrolyzed with distilled water $(0.45 \mathrm{~mL}$, $25 \mathrm{mmol})$. After 15 minutes, the reaction mixture was filtered by gravity to remove solid LiOH. The resultant solution of diethyl guanidine was concentrated and returned to the nitrogen filled glove box. The guanidine solution was cooled in the freezer and added to a solution of alane complexed with $\mathrm{N}, \mathrm{N}$-dimethylethylamine $(19.0 \mathrm{~mL}, 0.5 \mathrm{M}$ in toluene, $9.5 \mathrm{mmol}$ ) in a $250 \mathrm{~mL}$ round bottomed flask. The reaction was left to stir at room temperature over night. The 
solution was concentrated under vacuum and left to crystallize at $-30^{\circ} \mathrm{C}$. Clear, colourless crystals of compound 2 were collected by filtration $(4.48 \mathrm{~g}, 8.2 \mathrm{mmol}, 86 \%) . \mathrm{MP}: 117-119^{\circ} \mathrm{C} .{ }^{1} \mathrm{H}$ NMR (300 MHz, $\left.\mathrm{C}_{6} \mathrm{D}_{6}\right): 4.62$ [broad, $1 \mathrm{H}, \mathrm{Al}-\mathrm{H}$ ], 3.52 [sept, 4H, C(NCH(CH$\left.)_{2}\right)_{2}$ ], $2.96[\mathrm{q}, 8 \mathrm{H}$, $\left.\mathrm{CN}\left(\mathrm{CH}_{2} \mathrm{CH}_{3}\right)_{2}\right], 1.42\left[\mathrm{~d}, 24 \mathrm{H}, \mathrm{NCH}\left(\mathrm{CH}_{3}\right)_{2}\right], 0.89\left[\mathrm{t}, 12 \mathrm{H}, \mathrm{CN}\left(\mathrm{CH}_{2} \mathrm{CH}_{3}\right)_{2}\right] .{ }^{13} \mathrm{C} \mathrm{NMR}\left(300 \mathrm{MHz}, \mathrm{C}_{6} \mathrm{D}_{6}\right):$ $170.31\left[\mathrm{NC}\left(\mathrm{N}^{\prime} \mathrm{Pr}_{2}\right) \mathrm{N}\right], 47.16\left[\mathrm{CN}\left(\mathrm{CH}_{2} \mathrm{CH}_{3}\right)_{2}\right], 43.28\left[\mathrm{CNC}\left(\mathrm{CH}_{3}\right)_{2}\right], 25.98\left[\mathrm{CN}\left(\mathrm{CH}_{2} \mathrm{CH}_{3}\right)_{2}\right], 14.32$ [CNC(CH$\left.)_{2}\right] . M S: m / z(\%): 423.4(100)[M-1]$.

\subsection{4 ['PrHNC(N'Pr) $\left.{ }_{2}\right]_{2} A I H(3)$}

A solution of alane complexed with $\mathrm{N}, \mathrm{N}$-dimethylethylamine $(25.0 \mathrm{~mL}, 0.5 \mathrm{M}$ in toluene, $12.5 \mathrm{mmol}$ ) was diluted with toluene $(100 \mathrm{~mL})$ in a $250 \mathrm{~mL}$ round bottomed flask and placed in a freezer $\left(-30^{\circ} \mathrm{C}\right)$ to cool for 10 minutes. Triisopropyl guanidine $(4.19 \mathrm{~g}, 22.6 \mathrm{mmol})$ was dissolved in hexanes $(30 \mathrm{~mL})$ and transferred into a dropping funnel. The alane solution was removed from the freezer and left to warm up to room temperature as the ligand solution was added at a rate of 15 drops per minute. Once the addition was completed, the reaction mixture was left to stir at room temperature over night. The solution was concentrated under vacuum and left to crystallize at $-30^{\circ} \mathrm{C}$. Clear, colourless crystals of compound 3 were collected by filtration (4.18g, 10.5mmol, 93\%). MP: $110-111^{\circ} \mathrm{C} .{ }^{1} \mathrm{H}$ NMR (300 MHz, C6D6): 4.74 [broad, $1 \mathrm{H}, \mathrm{Al}-\mathrm{H}$ ], 3.54 [sept, 2H, $\left.\mathrm{HNCH}\left(\mathrm{Me}_{2}\right)\right], 3.51$ [sept, 4H, $\left.\mathrm{NCH}\left(\mathrm{Me}_{2}\right)\right], 1.42\left[\mathrm{~d}, 24 \mathrm{H}, \mathrm{CH}\left(\mathrm{CH}_{3}\right)_{2}\right], 0.89[\mathrm{~d}, 12 \mathrm{H}$, $\left.\mathrm{CH}\left(\mathrm{CH}_{3}\right)_{2}\right] .{ }^{13} \mathrm{C} N \mathrm{NMR}(300 \mathrm{MHz}, \mathrm{C6D} 6): 164.29$ [NC(NH'Pr)N], $46.58\left[\mathrm{CNC}\left(\mathrm{CH}_{3}\right)_{2}\right], 46.00$ $\left[\mathrm{CNHC}\left(\mathrm{CH}_{3}\right)_{2}\right], 25.51\left[\mathrm{CNC}\left(\mathrm{CH}_{3}\right)_{2}\right], 24.52\left[\mathrm{CNHC}\left(\mathrm{CH}_{3}\right)_{2}\right] . \mathrm{MS}: \mathrm{m} / \mathrm{z}(\%): 395(24)[\mathrm{M}-1]$.

\subsection{5 [Pr $\left.{ }_{2} \mathrm{NC}\left(\mathrm{N}^{\mathrm{P}} \mathrm{Pr}\right)_{2}\right]_{2} \mathrm{AIH}(4)$}

Diisopropylamine $(2.44 \mathrm{~g}, 24 \mathrm{mmol})$ was diluted with diethyl ether $(40 \mathrm{~mL})$ and placed in a freezer $\left(-30^{\circ} \mathrm{C}\right)$ to cool. To this solution, methyl lithium $(15 \mathrm{~mL}, 1.6 \mathrm{M}$ in ether, $23.3 \mathrm{mmol})$ was 
added dropwise slowly with stirring. After 1.5 hours, a solution of $N, N^{\prime}$-diisopropylcarbodiimide $(3.17 \mathrm{~g}, 24 \mathrm{mmol})$ diluted with diethyl ether $(15 \mathrm{~mL})$ was added dropwise to the reaction flask. The solution was left stirring overnight to form lithiated tetraisopropyl guanidinate. The reaction flask was removed from the nitrogen filled glove box and opened to atmosphere. To this, 1 equivalent of distilled water $(0.43 \mathrm{~mL}, 24 \mathrm{mmol})$ was added dropwise with stirring. After 15 minutes, clumped white $\mathrm{LiOH}$ was filtered off and the resulting guanidine solution was concentrated under vacuum. The flask was returned to the glove box where it was cooled in the freezer $\left(-30^{\circ} \mathrm{C}\right)$. In a $150 \mathrm{~mL}$ round bottomed flask, alane complexed with $\mathrm{N}, \mathrm{N}-$ dimethylethylamine $(24.0 \mathrm{~mL}, 0.5 \mathrm{M}$ in toluene, $12 \mathrm{mmol})$ was diluted with toluene $(30 \mathrm{~mL})$ and cooled to $-30^{\circ} \mathrm{C}$. The guanidine ligand solution was added dropwise to the alane solution slowly and left to stir over night. The volatiles were removed to isolate a yellow powder. The solid was recrystallized from toluene to yield colourless crystals of compound $4(4.50 \mathrm{~g}, 9.4 \mathrm{mmol}, 78 \%)$. MP: $112-113^{\circ} \mathrm{C} .{ }^{1} \mathrm{H}$ NMR $\left(300 \mathrm{MHz}, \mathrm{C}_{6} \mathrm{D}_{6}\right): 4.48$ [broad, $1 \mathrm{H}, \mathrm{Al}-\mathrm{H}$ ], 3.74 [sept, $4 \mathrm{H}, \mathrm{C}\left(\mathrm{NCH}\left(\mathrm{CH}_{3}\right)_{2}\right)_{2}$ ], 3.47 [sept, $\left.4 \mathrm{H}, \mathrm{HNCH}\left(\mathrm{CH}_{3}\right)_{2}\right], 1.44\left[\mathrm{~d}, 24 \mathrm{H}, \mathrm{C}\left(\mathrm{NCH}\left(\mathrm{CH}_{3}\right)_{2}\right)_{2}\right], 1.14\left[\mathrm{~d}, 24 \mathrm{H}, \mathrm{HNCH}\left(\mathrm{CH}_{3}\right)_{2}\right] .{ }^{13} \mathrm{C} \mathrm{NMR}$ $\left(300 \mathrm{MHz}, \mathrm{C}_{6} \mathrm{D}_{6}\right): 170.97\left[\mathrm{NC}\left(\mathrm{N}^{\prime} \mathrm{Pr} \mathrm{r}_{2}\right) \mathrm{N}\right], 50.46\left[\mathrm{NC}\left(\mathrm{CH}_{3}\right)_{2}\right], 47.00\left[\mathrm{CN}\left(\mathrm{C}\left(\mathrm{CH}_{3}\right)_{2}\right)_{2}\right], 26.27\left[\mathrm{NC}\left(\mathrm{CH}_{3}\right)_{2}\right]$, $24.31\left[\mathrm{CN}\left(\mathrm{C}\left(\mathrm{CH}_{3}\right)_{2}\right)_{2}\right] . \mathrm{MS}: \mathrm{m} / \mathrm{z}(\%): 479(100)[\mathrm{M}-1]$.

\subsection{6 $\left[\mathrm{MeC}\left(\mathrm{N}^{\mathrm{i} P r}\right)_{2}\right]_{2} \mathrm{AlH}(5)$}

In a $50 \mathrm{~mL}$ flask, $\left[\mathrm{MeC}\left(\mathrm{N}^{\prime} \mathrm{Pr}\right)_{2}\right]_{3} \mathrm{Al}(1.031 \mathrm{~g}, 3.3 \mathrm{mmol})$ was dissolved in toluene $(20 \mathrm{~mL})$ with stirring. A solution of alane complexed with $\mathrm{N}, \mathrm{N}$-dimethylethylamine $(15.0 \mathrm{~mL}, 0.5 \mathrm{M}$ in toluene, $7.5 \mathrm{mmol})$ diluted with toluene $(15 \mathrm{~mL})$ was added dropwise, in excess to $\left[\mathrm{MeC}\left(\mathrm{N}^{\prime} \mathrm{Pr}\right)_{2}\right]_{3} \mathrm{Al}$. The reaction was left to stir at room temperature for $66 \mathrm{~h}$ to reach a point of equilibrium. Volatiles were removed from the reaction flask and the resultant grey-white powder was transferred 
into a sublimation apparatus and heated to $53^{\circ} \mathrm{C}$ at $110 \mathrm{mTorr}$. Clear colourless crystals of compound $5(0.25 \mathrm{~g}, 0.56 \mathrm{mmol}, 16 \%)$ were collected from the condenser. M.P.: $50-51^{\circ} \mathrm{C} .{ }^{1} \mathrm{H}$ NMR (300 MHz, $\left.\mathrm{C}_{6} \mathrm{D}_{6}\right): 4.88$ [broad, $1 \mathrm{H}, \mathrm{Al}-\mathrm{H}$ ], 3.41 [sept, $4 \mathrm{H}, \mathrm{CH}\left(\mathrm{CH}_{3}\right)_{2}$ ] $, 1.48\left[\mathrm{~s}, 6 \mathrm{H}, \mathrm{CCH}_{3}\right], 1.27$ [d, 24H, $\left.\mathrm{CH}\left(\mathrm{CH}_{3}\right)_{2}\right] .{ }^{13} \mathrm{C}$ NMR $\left(300 \mathrm{MHz}, \mathrm{C}_{6} \mathrm{D}_{6}\right): 171.78\left[\mathrm{NC}\left(\mathrm{CH}_{3}\right) \mathrm{N}\right], 46.81\left[\mathrm{CH}\left(\mathrm{CH}_{3}\right)_{2}\right], 25.72$ $\left[\mathrm{N}\left(\mathrm{CH}_{3}\right)_{2}\right], 11.58\left[\mathrm{NC}\left(\mathrm{CH}_{3}\right) \mathrm{N}\right] . \mathrm{MS}: \mathrm{m} / 2(\%): 309.3(5.8)[\mathrm{M}-1]$.

\subsection{Deposition}

\subsubsection{Substrate Preparation}

Aluminum metal films were grown on p-type $\mathrm{Si}(100)$ test grade substrates purchased from University Wafer. The substrates were cut to size (approximately 1 to $1.5 \mathrm{~cm}$ wide) and cleaned prior to deposition experiments. Surface organics were removed from the substrates by sonication in acetone for 30 minutes in a 250D Aquasonic Ultrasonic Cleaner by VWR. The substrates were then rinsed with distilled water and immersed in an acid solution of a 1:10:10 ratio mixture of $\mathrm{H}_{2} \mathrm{O}_{2}: \mathrm{H}_{2} \mathrm{SO}_{4}: \mathrm{H}_{2} \mathrm{O}$ for 30 minutes, followed by a rinse with distilled water and then sonication in distilled water for 10 minutes. Next the substrates were dipped in a series of acid baths, intermittently rinsing with distilled water, in order to clean and etch the silicon surface: first, a 4:1 ratio of $\mathrm{H}_{2} \mathrm{SO}_{4}: \mathrm{H}_{2} \mathrm{O}_{2}$ for 10 minutes, second, a 1:1:6 ratio of $\mathrm{NH}_{4} \mathrm{OH}: \mathrm{H}_{2} \mathrm{O}_{2}: \mathrm{H}_{2} \mathrm{O}$ for 10 minutes, and last, a 1:1:6 mixture of $\mathrm{HCl}: \mathrm{H}_{2} \mathrm{O}_{2}: \mathrm{H}_{2} \mathrm{O}$ for 10 minutes. The substrates were air dried and then subjected to $\mathrm{UV} / \mathrm{O}_{3}$ in a UVO-cleaner (model 42 by Jelight company Inc.) for 30 seconds to grow a native oxide layer on the $\mathrm{Si}(100)$ surface. The substrates were stored in a furnace just under $100^{\circ} \mathrm{C}$. Three substrates were positioned in the ALD deposition chamber close to the injection point and placed under vacuum approximately 1 hour prior to the commencement of each deposition. 


\subsubsection{Pulsed Chemical Vapour Deposition}

Compound $\mathbf{3}$ was weighed into a vial and sealed into a stainless steel vessel, or 'bubbler', via a CF fitting within a nitrogen filled glovebox. The bubbler was removed from the glovebox and positioned in line with valve 4 of the reactor valve array where a second CF fitting was used to connect the bubbler to the line providing an air tight seal. The line connecting the bubbler to the valve array was purged by alternating vacuum and nitrogen gas 3 times to remove any atmosphere trapped in the line before the manual valve on the bubbler was opened. The atmosphere of the bubbler was also purged and then left under fresh nitrogen to ensure any oxygen leaks in the set up process be rectified. On the line extending from valve 5 , a rubber hose was used to connect a tank of forming gas $\left(5 \% \mathrm{H}_{2}\right.$ in $\left.\mathrm{N}_{2}\right)$ behind a manual valve. The line up to the valve and the hose to the regulator were each purged in a similar manner to the bubbler. The regulator was then turned on to fill the hose between the regulator and the now closed manual valve.

Using He gas, the apparatus was leak tested using the residual gas analyzer (RGA) to track any increases in He gas $(\mathrm{m} / \mathrm{z}=4 \mathrm{u})$ passing through the reactor. This along with a sufficient reactor base pressure of $<100 \mathrm{mTorr}$ were adequate to assure the integrity of the system and continue with the depositions. The lines and bubbler were then wrapped with thermal heating tape. This procedure was performed each time the precursor bubbler was reloaded between deposition runs.

The $\mathrm{H}_{2}$ flow was adjusted using the manual valve such that the system pressure remained even at about $475 \mathrm{mTorr}$. This value was selected as a means of gaining uniformity in flow between deposition runs but should not be thought of as an optimized parameter. 
Substrates were loaded into the reaction furnace on a copper holder and heated under vacuum to deposition temperature $\left(250-350^{\circ} \mathrm{C}\right)$. The heating tape surrounding the bubbler was heated to desired temperature $\left(95^{\circ} \mathrm{C}\right)$ and subsequent heating zones were also heated, stepping up $10^{\circ} \mathrm{C}$ each zone toward the deposition furnace to avoid condensation of precursor in the lines of the apparatus during the deposition run. The manual valve on the bubbler was then opened.

The deposition program used to control the opening and closing of the valves in the valve array was then set up and the deposition run was commenced. First a 5 second purge of nitrogen was allowed to pass through the deposition chamber followed by 5 seconds of vacuum. Nitrogen gas was then introduced to the bubbler and then allowed to pass through, carrying the precursor compound, to the deposition chamber. Fresh nitrogen was then allowed to flow through the chamber for 5 seconds to remove excess precursor and deposition products. A flow of forming gas was then brought through the reaction chamber to reduce the surface species, followed by a period of vacuum to clear the reaction chamber. The entire pulse sequence was repeated for a desired number of cycles. Detailed parameters used for each deposition can be found in Appendix C. 


\section{REFERENCES}

${ }^{1}$ Moore, G.E., Electronics, 1965, 38(8), 114-117.

${ }^{2}$ Moore, G. E. Excerpts from a conversation with Gordon Moore: Moore's Law. Intel Corporation, 2005.

${ }^{3}$ Leskela, M. and Ritala, M., Angew. Chem. Int. Ed., 2003, 42, 5548-5554.

${ }^{4}$ Volksen, W., Miller, R.D. and Dubois, G. Chem. Rev., 2010, 110, 56-110.

${ }^{5}$ Rickerby, J. and Steinke, J.H.G., Chem. Rev., 2002, 102, 1525-1549.

${ }^{6}$ Kim, H.-C., Park, S.-M. and Hinsberg, W.D. Chem. Rev., 2010, 110, 146-177.

${ }^{7}$ Lide, D.R. CRC Handbook of Chemistry and Physics, 86th Ed. Boca Raton, CRC Press, Taylor \& Francis Group, 2006.

${ }^{8}$ Vargel, C. Corrosion of Aluminum. Kidlington, Oxford: Elsevier Ltd., 2004.

${ }^{9}$ Kim, H., j. Vac. Sci. Technol. B, 2003, 21(6), 2231-2261.

${ }^{10}$ Kim, H.; Detavenier, C.; van der Straten, O.; Rossnagel, S.M.; Kellock, A.J.; Park, D.-G., J. Appl. Phys., 2005, 98(1), 14308-14316.

${ }^{11}$ Romang, A.H. and Watkins, J.J., Chem. Rev., 2010, 110, 459-478.

${ }^{12}$ Jensen, K.F. and Kern, W. (Eds.). Thin Film Processes II. New York : Academic Press, 1991.

${ }^{13}$ Yuan, C.Y. and Dornfeld, D. IEEE International Symposium on Electronics and the Environment, San Francisco, CA : 2008.

${ }^{14}$ Crowell, J.E., J. Vac. Sci. Technol. A, 2003, 21, S88.

${ }^{15}$ Suntola, T. and Antson, J. Method for Producing Compound Films. US patent, No. 4,058,430 November 15, 1977.

${ }^{16}$ Suntola, T and Hyvarinen, J. Ann. Rev. Meter. Sci., 1985, 15, 177-95.

${ }^{17}$ Leskala, M. and Ritala, M. Journal de Physique IV, 1995, 5(11), 937-951.

${ }^{18}$ George, S.M., Chem. Rev., 2010, 110, 111-131.

${ }^{19}$ Lee, Y.J. and Kang, S.-W., Electrochemical and Solid-State Letters, 2002, 5(10), C91-C93.

${ }^{20}$ Gordon, R.; Hausmann, D.; Kim, E.; Shepard, J., Chem. Vap. Deposition, 2003, 9(2), 73-78. 
${ }^{21}$ Leskela, M. and Ritala, M., J. Phys. IV France, 1999, 9, 837-852.

${ }^{22}$ Leskela, M. and Ritala, M., Chem. Vap. Deposition, 2003, 9(1), 45-49.

${ }^{23}$ Utriainen, M.; Kroger-Laukkanen, M.; Johansson, L.; L., Niinistom, Appl. Surf. Sci., 2000, 157, 151-158.

${ }^{24}$ Shriner, R.L. and Neumann, F.W., Chem. Rev., 1944, 35(3), 351-425.

${ }^{25}$ Brazeau, A.L. Theoretical and Experimental Investigations of Ligand Exchange in Amidinate and Guanidinate Ligand Systems and the Atomic Layer Deposition of Aluminum Oxide, MSc Thesis; Carleton University Press, Ottawa, ON, 2007.

${ }^{26}$ Delahunt, J. R., A Thermolysis Study of Aluminum Acetamidinates, Guanidinates, and Formamidinates. Poster presentation: 40th Inorganis Discussion Weekend, Toronto, ON, 2007.

${ }^{27}$ Coyle, J.P.; Monillas, W.H.; Yap, G.P.A.; Barry, S.T., Inorg. Chem. , 2008, 47, 683-689.

${ }^{28}$ Ziffle, L.C.; Kenney, A.P.; Barry, S.T.; Muller, J., Polyhedron, 2008, 27, 1832-1840.

${ }^{29}$ Coyle, J.P.; Johnson, P.A.; DiLabio, G.A.; Barry, S.T.; Muller, J., Inorg. Chem. , 2010, 49, 28442850.

${ }^{30}$ Serp, P and Kalck, P., Chemical Reviews, 2002, 102, 3085.

${ }^{31}$ Gunter, P.L.; Niemantsverdriet, W.J.H.; Ribeiro, F.H.; Somorjai, G.A., Catal. Rev.-Sci. Eng., 1997, 39(77).

${ }^{32}$ Sindorf, D.W. and Maclel, G.E., J. Phys. Chem. , 1983, 87, 5516-5521.

${ }^{33}$ Chae, J., Park, H.S. and Kang, S.W., Electrochem. Solid-State Lett., 2002, 5(6), C64-66.

${ }^{34}$ George, S.M., Ott, A.W. and Klaus, J.W., J. Phys. Chem., 1996, 100, 13121-13131.

${ }^{35}$ Greenwood, N.N. and Earnshaw, A. Chemistry of the elements. Oxford : ButterworthHeinemann, 1997.

${ }^{36}$ Aldridge, S. and Downs, A.J., Chem. Rev. , 2001, 101, 3305-3365.

${ }^{37}$ Kaesz, H.D. and Saillant, R.B., Chem. Rev., 1972, 72(3), 231-281.

${ }^{38}$ Frigo, D. M.; van Eijden, G. J. M.; Reuvers, P. J.; Smit, C.J., Chem. Mater., 1994, 6(2), 190-195.

${ }^{39}$ Cole, M. L.; Jones, C.; Junk, P.C.; Marc Kloth, M.; Stasch, A., Chem. Eur. J., 2005, 11, 4482 4491. 
${ }^{40}$ Himmel, H.J., Inorg. Chem., 2007, 46(16), 6585-6593.

${ }^{41}$ Delahunt, J.R. Aluminum Metal Thin Films by Vapour Deposition. Ottawa : BSc Thesis; Carleton University Press, Ottawa, ON, 2008.

${ }^{42}$ Feil, F. and Harder, S., Eur. J. Inorg. Chem., 2005, 4438-4443.

${ }^{43}$ Bailey, P.J. and Pace, S., Coord. Chem. Rev., 2001, 214, 91-141.

${ }^{44}$ Kurzer, F. and Douraghi-Zadeh, K., Chem. Rev., 1967, 7(2), 107-152.

${ }^{45}$ Khorana, H. G., Chem. Rev., 1953, 145-165.

${ }^{46}$ Brazeau, A.L.; Wang, Z.; Rowley, C.N.; Barry, S.T. , Inorg. Chem. , 2006, 45, 2276-2281.

${ }^{47}$ Kenney, A. P.; Yap, G. P. A.; Richeson, D. S.; Barry, S. T., Inorg. chem., 2005, 44, 2926.

${ }^{48}$ Brazeau, A.L.; DiLabio, G.A.; Kreisel, K.A.; Monillas, W.; Yap, G.P.A.; Barry, S.T. , Dalton Trans., 2007, 3297-3304.

${ }^{49}$ Rowley, C. N., DiLabio, G. A. and Barry, S. T., Inorg. Chem., 2005, 44, 1983-1991.

${ }^{50}$ Aaltonen, T.; Alen, P.; Ritala, M.; Leskela, M., Chem. Vap. Deposition, 2003, 9(1), 45-49.

${ }^{51}$ Addison, A. W.; Rao, T. N.; Reedijk, J.; van Rijin, J.; Verschoor, G. C., J. Chem. Soc., Dalton Trans., 1984, 7, 1349-1356.

${ }^{52}$ Russegger, P.; Brickmann, J., Chem. Phys. Let. 2, s.l. : chemical physica letters, 1975, 30(2), 276-278.

${ }^{53}$ Downs, R.T. and Hall-Wallace, M. The American Mineralogist Crystal Structure Database. [Online] Department of Geosciences, University of Arizona, Tucson, Arizona, 2003. <http://rruff.geo.arizona.edu/AMS/amcsd.php>.

${ }^{54}$ Wycoff, R.W.G. Crystal Structures. 2nd Ed. . New York, New York : Interscience Publishers, 1963. Vol. 1, pp 7-83.

${ }^{55}$ Wagner, C.D., J. Vac. Sci. Technol., 1978, 15(2). 518-523.

${ }^{56}$ Taylor, J.A., Appl. Surf. Sci., 1981, 7, 168-184.

${ }^{57}$ Waggoner, K. M., Olmstead, M. M. and Power, P.P., Polyhedron, 1990, 9, 257.

${ }^{58}$ Foley, S. R., Yap, G. P. A. and Richeson, D. S., Chem. Commun., 2000, 16, 1515. 


\section{APPENDICES:}




\section{Appendix A: Structural Determination by Single Crystal XRD}

Selected crystal data and structural refinement parameters for compounds $1,2,4$, and $5 \ldots . . . A-1$ Selected geometric parameters for compounds 1, 2, 4, and 5

\section{Compound 1: $\left[\mathrm{Me}_{2} \mathrm{NC}\left(\mathrm{N}^{\mathrm{i}} \mathrm{Pr}\right)_{2}\right]_{2} \mathrm{AlH}$}

-Selected structural views.

-Full crystallographic information '.cif'

A-4 to $A-15$

\section{Compound 2: $\left[\mathrm{Et}_{2} \mathrm{NC}\left(\mathrm{N}^{\mathrm{i} P r}\right)_{2}\right]_{2} \mathrm{AlH}$}

-Selected structural views

A-16

-Full crystallographic information '.cif'

A-17 to $A-26$

\section{Compound 4: $\left[{ }^{i} \mathrm{Pr}_{2} \mathrm{NC}\left(\mathrm{N}^{\mathrm{i}} \mathrm{Pr}\right)_{2}\right]_{2} \mathrm{AlH}$}

-Selected structural views

-Full crystallographic information '.cif'

A-28 to $A-44$

\section{Compound 5: $\left[\mathrm{MeC}\left(\mathrm{N}^{\mathrm{i}} \mathrm{Pr}\right)_{2}\right]_{2} \mathrm{AlH}$}

-Selected structural views.

-Full crystallographic information '.cif'

$A-46$ to $A-52$ 
Data Tables for compounds 1, 2, 4, and 5:

Table 10. Selected crystal data and structural refinement parameters for compounds 1, 2, 4, and 5

\begin{tabular}{|c|c|c|c|c|}
\hline & Compound 1 & Compound 2 & Compound 4 & Compound 5 \\
\hline fw & 368.55 & 424.38 & 480.76 & 310.46 \\
\hline$T(\mathrm{~K})$ & 206 & 200 & 200 & 200 \\
\hline cryst syst & Monoclinic & Orthorhombic & Triclinic & Monoclinic \\
\hline space group & $\mathrm{p} 2(1) / c$ & $\mathrm{p} 2(1) 2(1) 2(1)$ & P-1 & $\mathrm{C} 2 / \mathrm{c}$ \\
\hline$c(\AA)$ & 19.03 & 17.00 & 19.04 & 14.19 \\
\hline$\alpha$ (deg) & 90 & 90 & 81.06 & 90 \\
\hline$B$ (deg) & 98.11 & 90 & 84.35 & 107.87 \\
\hline$v$ (deg) & 90 & 90 & 89.66 & 90 \\
\hline abs coeff $\left(\mathrm{mm}^{-1}\right)$ & 0.099 & 0.091 & 0.086 & 0.104 \\
\hline refinement method & $\begin{array}{l}\text { Full-matrix least-squares } \\
\text { on } \mathrm{F}^{2}\end{array}$ & $\begin{array}{l}\text { Full-matrix least-squares } \\
\text { on } \mathrm{F}^{2}\end{array}$ & $\begin{array}{l}\text { Full-matrix least-squares } \\
\text { on } \mathrm{F}^{2}\end{array}$ & $\begin{array}{l}\text { Full-matrix least-squares } \\
\text { on } \mathrm{F}^{2}\end{array}$ \\
\hline $\begin{array}{l}R \text { indices } \\
{[1>2 \sigma(1)]^{*}}\end{array}$ & $\begin{array}{l}R 1=0.0632 \\
W R 2=0.1408\end{array}$ & $\begin{array}{l}R 1=0.0442, \\
W R 2=0.1195\end{array}$ & $\begin{array}{l}R 1=0.0607 \\
W R 2=0.1450\end{array}$ & $\begin{array}{l}R 1=0.0440 \\
W R 2=0.1286\end{array}$ \\
\hline
\end{tabular}




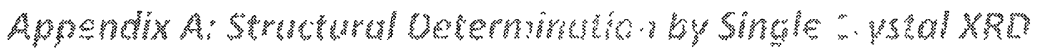

Table 2. Selected geometric parameters for compounds 1, 2, 4, and 5

\begin{tabular}{|c|c|c|c|c|}
\hline & Compound 1 & Compound 2 & Compound 4 & Compound 5 \\
\hline \multicolumn{5}{|l|}{ Bond Length ( $(\AA)$} \\
\hline Al-H1 & 1.58 & 1.53 & 1.51 & 1.94 \\
\hline Al-N1 & 2.00 & 2.03 & 2.03 & 2.06 \\
\hline Al-N2 & 1.95 & 1.94 & 1.93 & 1.94 \\
\hline Al-N4 & 2.00 & 2.03 & 2.06 & \\
\hline Al-N5 & 1.95 & 1.94 & 1.93 & \\
\hline \multicolumn{5}{|l|}{ Bond Angle (deg) } \\
\hline N1-Al-N2 & 67.8 & 67.5 & 67.4 & 103.0 \\
\hline N4-Al-N5 & 67.8 & 67.4 & 67.1 & 161.7 \\
\hline N2-Al-N4 & 102.3 & 103.2 & 103.5 & \\
\hline N1-Al-N5 & 102.7 & 105.1 & 102.3 & \\
\hline N2-Al-N5 & 116.7 & 163.8 & 161.9 & \\
\hline N1-Al-N4 & 162.3 & 120.5 & 117.6 & 66.7 \\
\hline N1-Al-H1 & 100.5 & 96.7 & 98.2 & 99.2 \\
\hline N2-Al-H1 & 122.9 & 118.5 & 119.4 & 122.3 \\
\hline N4-Al-H1 & 97.2 & 99.5 & 99.9 & \\
\hline N5-Al-H1 & 120.4 & 121.0 & 123.0 & \\
\hline \multicolumn{5}{|l|}{ Torsion Angle (deg) } \\
\hline Al-N1-C1-N2 & 0.8 & 4.4 & 1.7 & 1.1 \\
\hline Al-N4-C2-N5 & 1.8 & 3.9 & 3.2 & 0.7 \\
\hline \multicolumn{5}{|c|}{ Sum of All Angles (deg) } \\
\hline IN3 & 358.9 & 358.9 & 358.9 & 358.9 \\
\hline$\Sigma N 6$ & 357.3 & 357.3 & 357.3 & 357.3 \\
\hline
\end{tabular}




\section{Compound 1: $\left[\mathrm{Me}_{2} \mathrm{NC}\left(\mathrm{N}^{\mathrm{i}} \mathrm{Pr}\right)_{2}\right]_{2} \mathrm{AlH}$}

View perpendicular to $\mathrm{Al}-\mathrm{H} 1$ bond

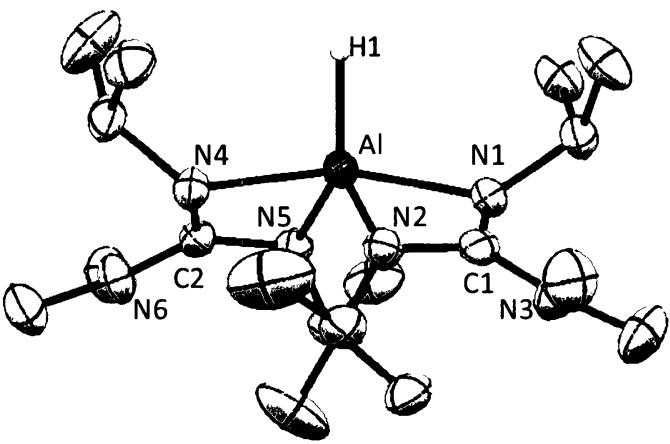

View along $\mathrm{Al}-\mathrm{H} 1$ bond

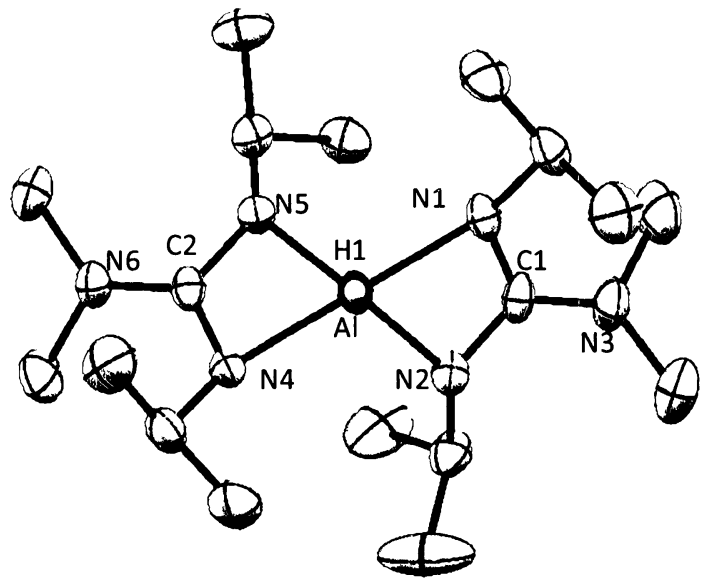

View of unit cell along a axis

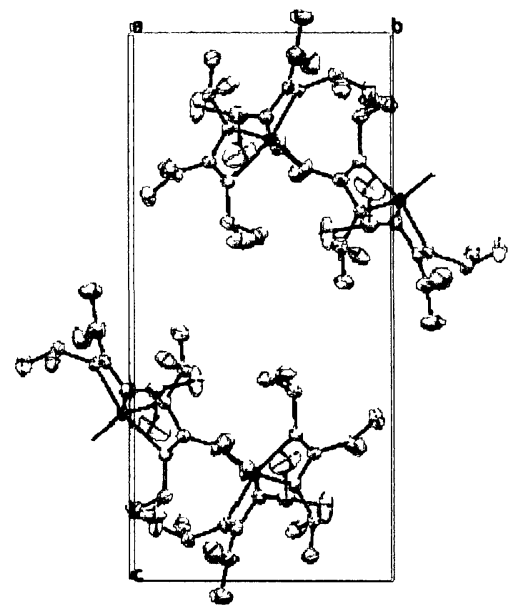

View of unit cell along $b$ axis

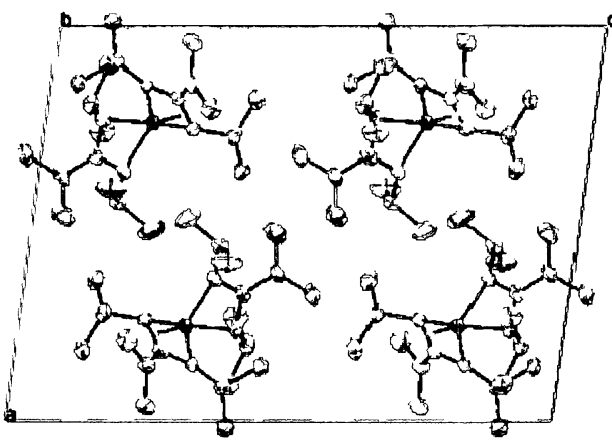

View of unit cell along $c$ axis

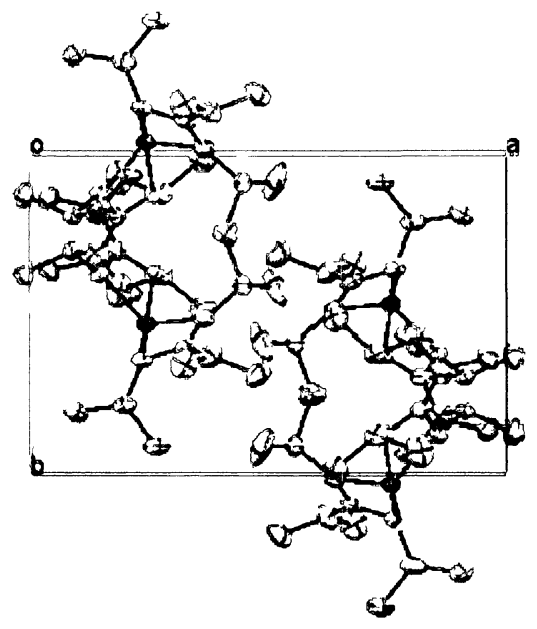




\section{Compound 1: $\left[\mathrm{Me}_{2} \mathrm{NC}\left(\mathrm{N}^{i} \mathrm{Pr}\right)_{2}\right]_{2} \mathrm{AlH}$.cif file}

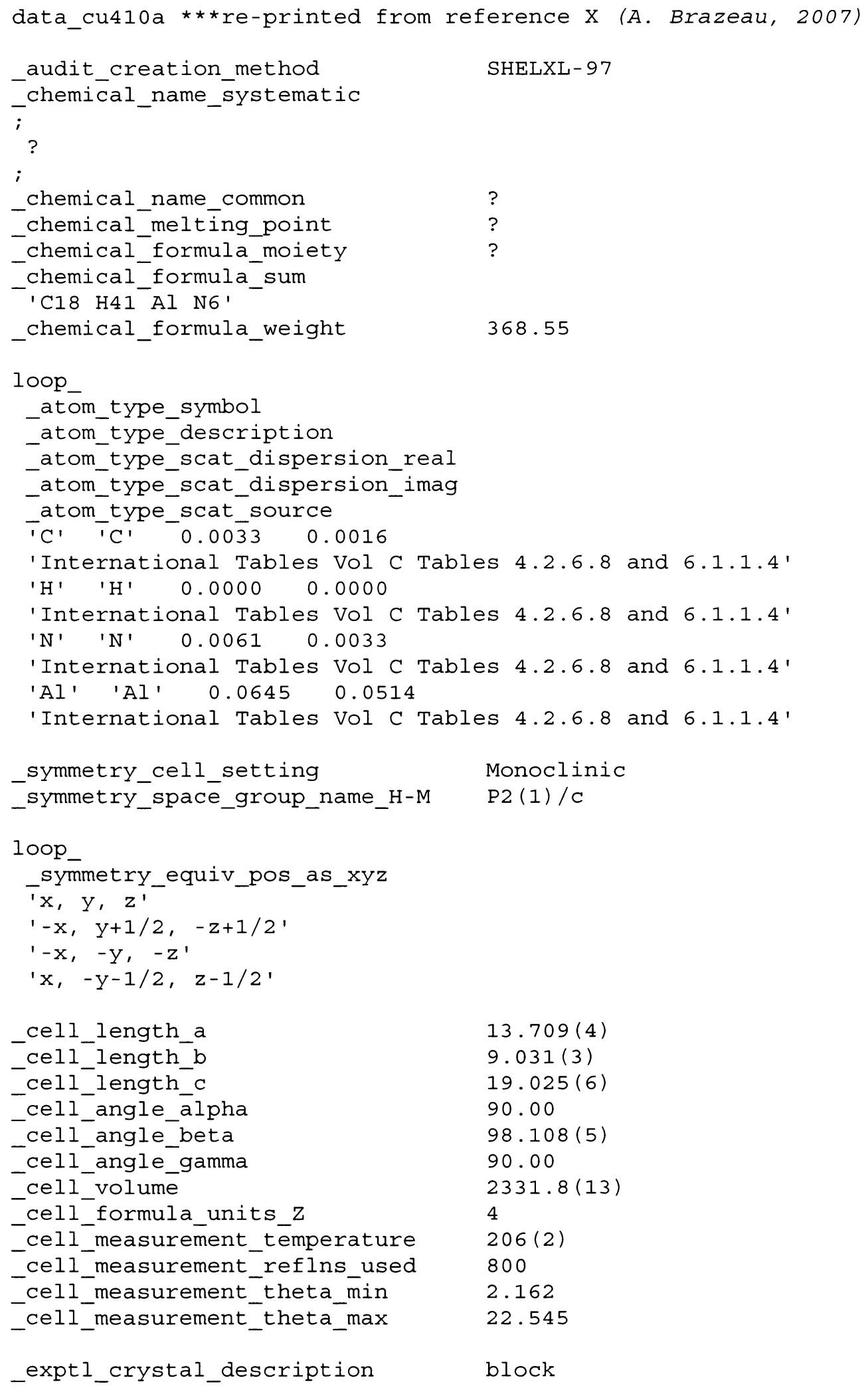




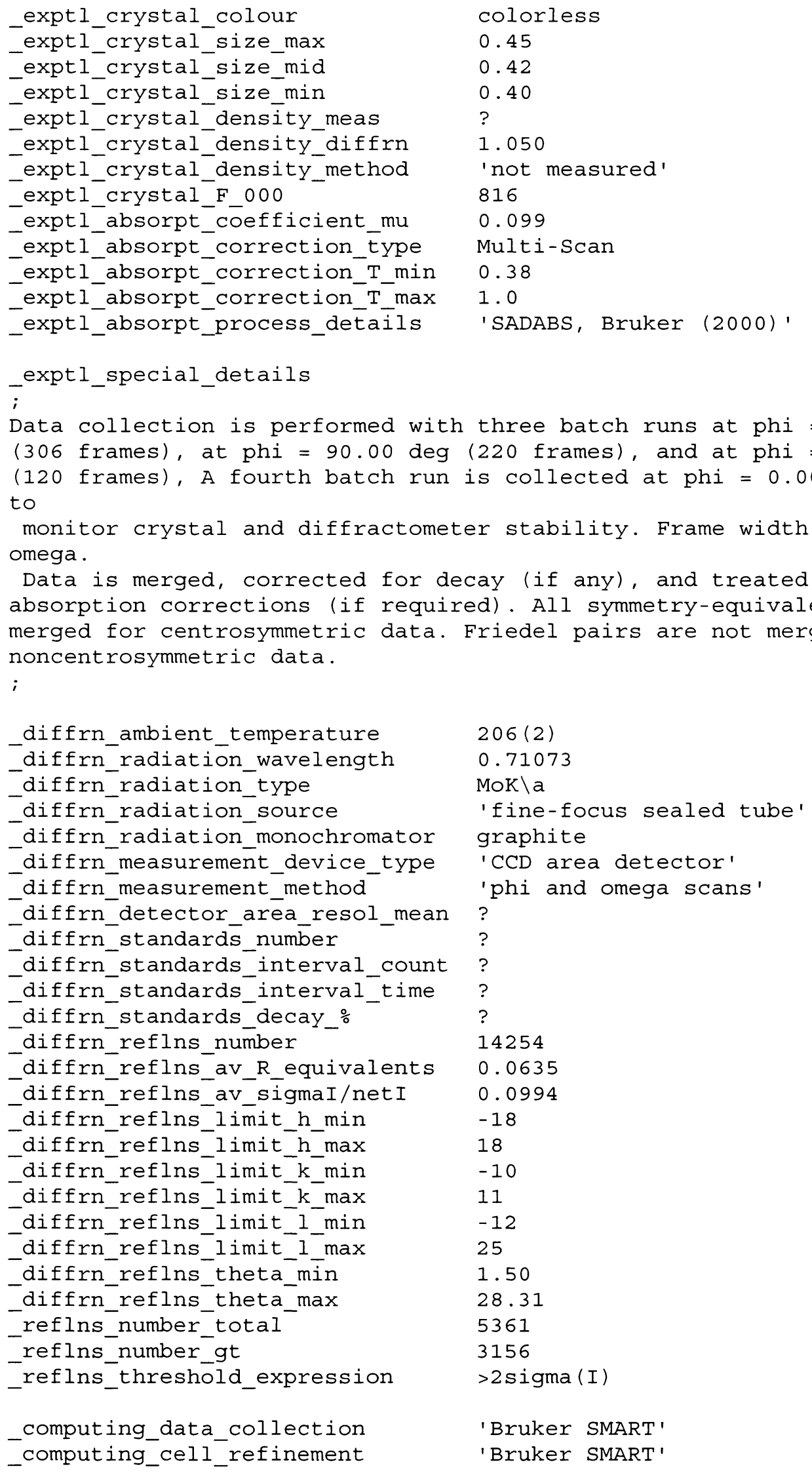




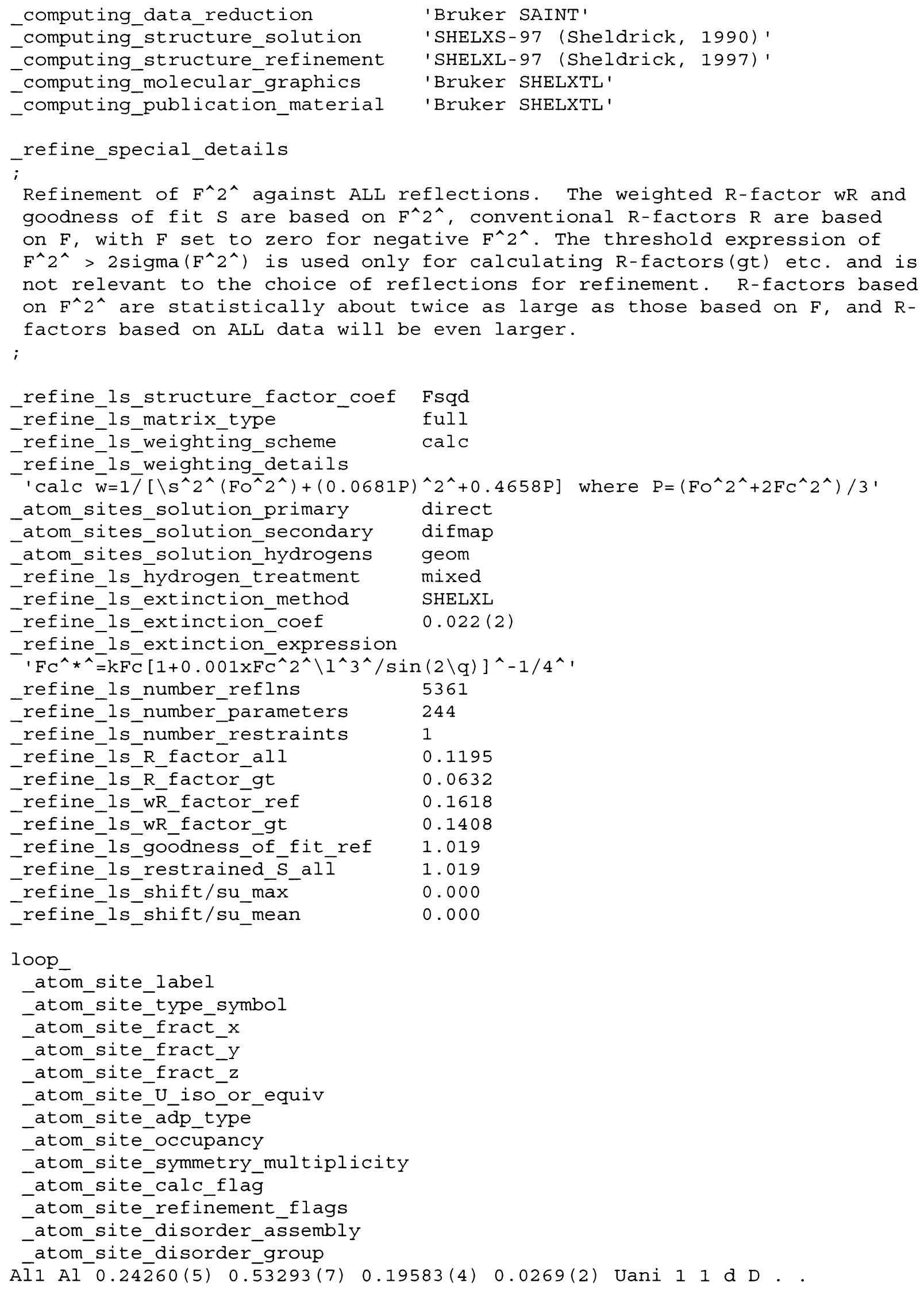


H2 $\mathrm{H} \quad 0.2228(16) \quad 0.664(2) \quad 0.2467(10) \quad 0.034(6)$ Uiso $11 \mathrm{~d} D$. N3 N $0.14599(13) \quad 0.37551(19) \quad 0.17441(9) \quad 0.0266(4)$ Uani $11 d .$. C4 C $0.18089(17) \quad 0.3016(2) \quad 0.23375(12) \quad 0.0279(5)$ Uani $11 d$. . N5 N $0.25838(14) \quad 0.3732(2) \quad 0.26899(10) \quad 0.0290(4)$ Uani $11 d . .$. N6 N $0.14418(15) \quad 0.1695(2) \quad 0.25428(10) \quad 0.0359(5)$ Uani $11 d . .$. C7 C $0.0394(2) \quad 0.1387(3) \quad 0.24680(15) \quad 0.0473(7) \quad$ Uani $11 d$. . H7A H 0.00290 .22520 .22770 .071 Uiso 11 calc $R$. H7B H $0.0210 \quad 0.1147 \quad 0.29290 .071$ Uiso 11 calc $R$. .

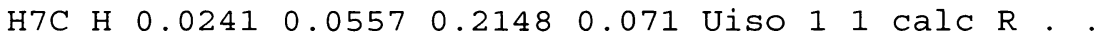
C8 C $0.2067(2) \quad 0.0640(3) \quad 0.29719(15) \quad 0.0480(7) \quad \operatorname{Uani} 11$ d. . . H8A H $0.1890-0.0358 \quad 0.28150 .072$ Uiso 11 Calc R. . H8B H $0.19790 .07490 .3466 \quad 0.072$ Uiso 1 I calc R. .

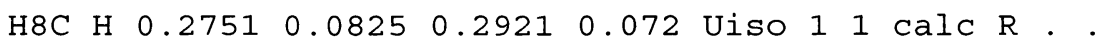
C9 C $0.27603(19) \quad 0.3780(3) \quad 0.34757(12) \quad 0.0392(6)$ Uani 11 d. . H9A H $0.2954 \quad 0.2778 \quad 0.36550 .047$ Uiso 11 Calc $R$. . C10 C $0.1843(2) \quad 0.4253(4) \quad 0.37848(15) \quad 0.0636(9)$ Uani 11 d . . H10A H 0.13130 .35620 .36360 .095 Uiso 11 calc $R$. . H10B H $0.16490 .5238 \quad 0.3616 \quad 0.095$ Uiso 11 Calc R. . H10C H $0.19850 .4261 \quad 0.42990 .095$ Uiso 11 calc R. . C11 C $0.3604(2) \quad 0.4835(3) \quad 0.37039(14) \quad 0.0484(7) \quad \operatorname{Uani} 11$ d. . H11A H $0.41810 .45170 .3503 \quad 0.073$ Uiso 11 Calc R . H11B H $0.3751 \quad 0.48390 .42180 .073$ Uiso 11 calc R . H1IC H $0.3420 \quad 0.58250 .35370 .073$ Uiso 11 calc R . . C12 C $0.08860(17) \quad 0.3029(2) \quad 0.11248(12) \quad 0.0313(6)$ Uani $11 d .$. H12A H 0.08150 .19670 .12380 .038 Uiso 11 calc R . . C13 C $-0.01415(19) \quad 0.3700(3) \quad 0.09572(14) \quad 0.0483(7)$ Uani $11 d .$. H13A H -0.04890 .35830 .13640 .072 Uiso 11 calc R. . H13B H -0.05020 .31990 .05500 .072 Uiso 11 calc $R$. . $\mathrm{H} 13 \mathrm{C} \mathrm{H}-0.00870 .47440 .08510 .072$ Uiso 11 calc $\mathrm{R}$. C14 C $0.1412(2) \quad 0.3144(3) \quad 0.04713(13) \quad 0.0417(7)$ Uani 11 d . . H14A H $0.2067 \quad 0.27250 .0578 \quad 0.063$ Uiso 11 Calc R. . H14B H $0.1460 \quad 0.4176 \quad 0.0340 \quad 0.063$ Uiso 11 calc R. $\mathrm{H} 14 \mathrm{C}$ H 0.10410 .26030 .00810 .063 Uiso 11 calc R . N15 N $0.36378(14) \quad 0.5154(2) \quad 0.15368(10) 0.0314(5)$ Uani $11 d$. C16 C $0.32644(18) \quad 0.5993(2) \quad 0.09791(12) \quad 0.0314(6)$ Uani $11 d . .$. $\mathrm{N} 17 \mathrm{~N} \quad 0.23451(15) \quad 0.64103(19) \quad 0.10289(10) 0.0305(5)$ Uani $11 d$.

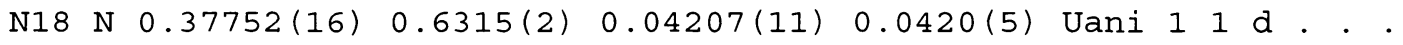
C19 C $0.4773(2) \quad 0.6879(3) \quad 0.05400(17) \quad 0.0615$ (9) Uani 11 d . . H19A H 0.50100 .68800 .10450 .092 Uiso 11 Calc R. . H19B H $0.47830 .78810 .0358 \quad 0.092$ Uiso 11 calc $R$. . H19C H 0.51940 .62530 .02970 .092 Uiso 11 calc R . . C20 C $0.3238(2) \quad 0.6552(4) \quad-0.02874(14) \quad 0.0599(9)$ Uani 11 d . . H2OA H $0.36350 .6213-0.0640 \quad 0.090$ Uiso 11 calc $R$. H2OB H $0.3098 \quad 0.7599-0.0357 \quad 0.090$ Uiso 11 CalC R. H20C H $0.26240 .6003-0.03370 .090$ Uiso 11 calc R. . C21 C $0.1958(2) \quad 0.7859(2) \quad 0.07675(14) \quad 0.0384(6)$ Uani 11 d. . H21A H 0.18790 .78570 .02420 .046 Uiso 11 Calc R . . C22 C $0.0954(2) \quad 0.8071(3) \quad 0.10023(15) \quad 0.0482(7) \quad \operatorname{Uani} 11$ d. . H22A H $0.0528 \quad 0.72540 .08300 .072$ Uiso 11 calc $\mathrm{R}$. . H22B H $0.0668 \quad 0.8994 \quad 0.08100 .072$ Uiso 11 calc $\mathrm{R}$. . $\mathrm{H} 22 \mathrm{C}$ H $0.1026 \quad 0.81030 .1517 \quad 0.072$ Uiso 1 l calc $\mathrm{R}$. . C23 C $0.2648(2) 0.9124(3) \quad 0.10436(18) \quad 0.0589$ (8) Uani 11 d... H23A H $0.3267 \quad 0.90220 .08560 .088$ Uiso 11 Calc R. . H23B H $0.27700 .9090 \quad 0.15580 .088$ Uiso 11 calc $R$. H23C H 0.23461 .00630 .08920 .088 Uiso 11 Calc R. C24 C $0.44483(18) \quad 0.4104(3) \quad 0.15091(14) \quad 0.0383(6)$ Uani 11 d . . H24A H 0.48130 .44130 .11200 .046 Uiso 11 Calc R A 1 
C25 C $0.4077(2) \quad 0.2553(3) \quad 0.1354(2) \quad 0.0710(10)$ Uani 11 d. B. H25A H 0.36990 .25180 .08830 .107 Uiso 11 Calc $R$. . H25B H 0.36610 .22620 .17020 .107 Uiso 11 Calc $R$. $\mathrm{H} 25 \mathrm{C} \mathrm{H} 0.46310 .18790 .1376 \quad 0.107$ Uiso 1 l calc R . . C26 C $0.5154(2) \quad 0.4143(5) \quad 0.2192(2) 0.0929(14)$ Uani 11 d 1 $\begin{array}{lllllllllllll}\mathrm{H} 26 \mathrm{~A} & \mathrm{H} & 0.5391 & 0.5147 & 0.2283 & 0.139 & \text { Uiso } 1 & 1 & \mathrm{CalC} & \mathrm{R} & \mathrm{B} & 1\end{array}$ $\begin{array}{lllllllllllllll}\mathrm{H} 26 \mathrm{~B} & \mathrm{H} & 0.5707 & 0.3494 & 0.2154 & 0.139 & \text { Uiso } 1 & 1 & \mathrm{calC} & \mathrm{R} & \mathrm{B} & 1\end{array}$

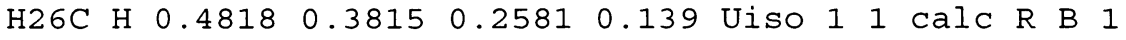

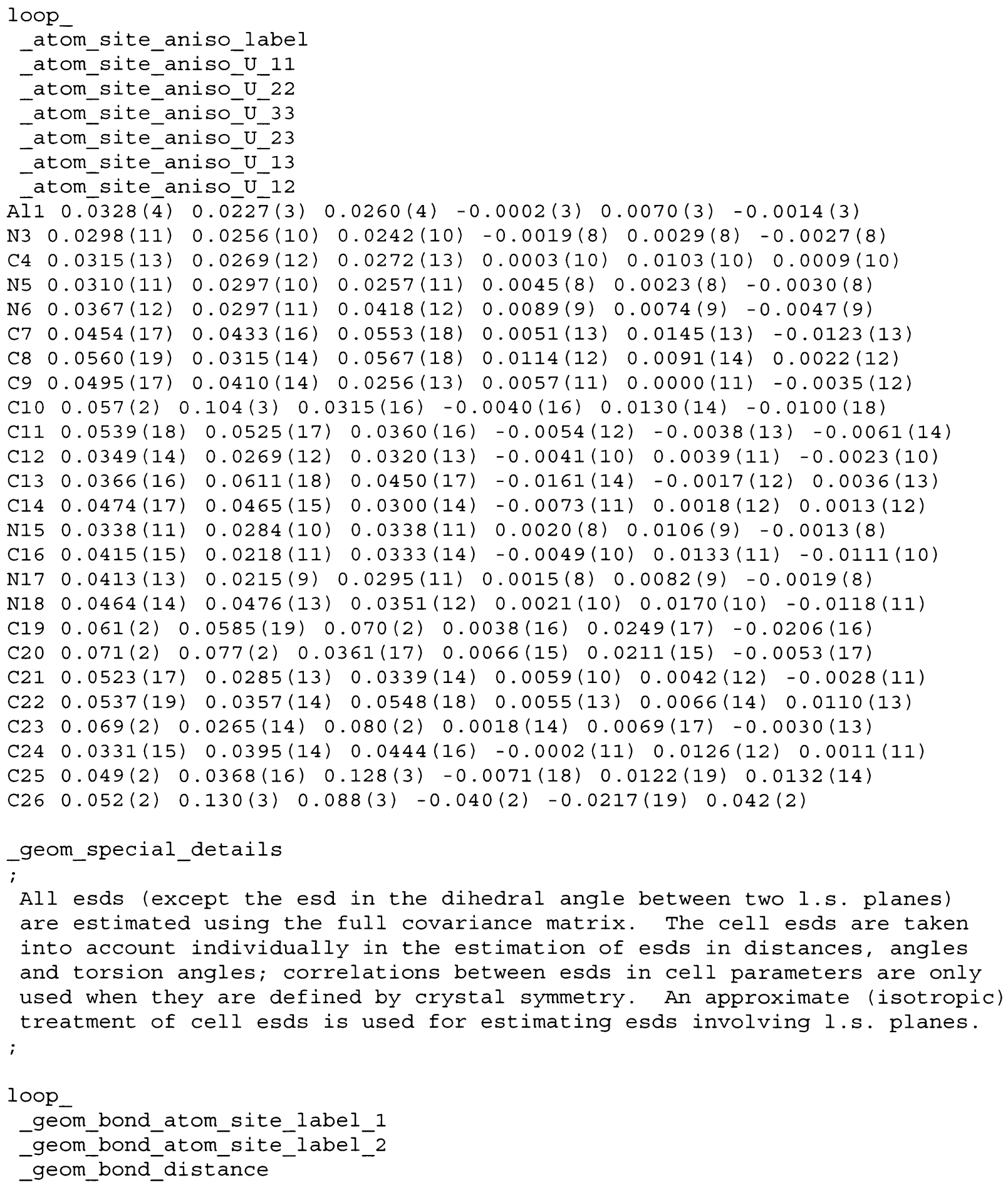




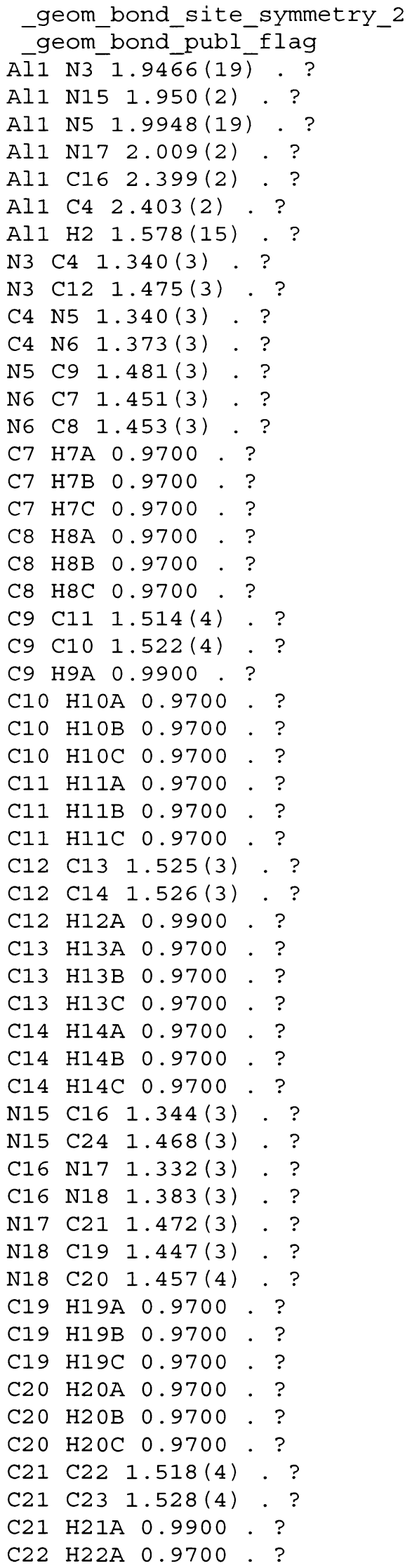




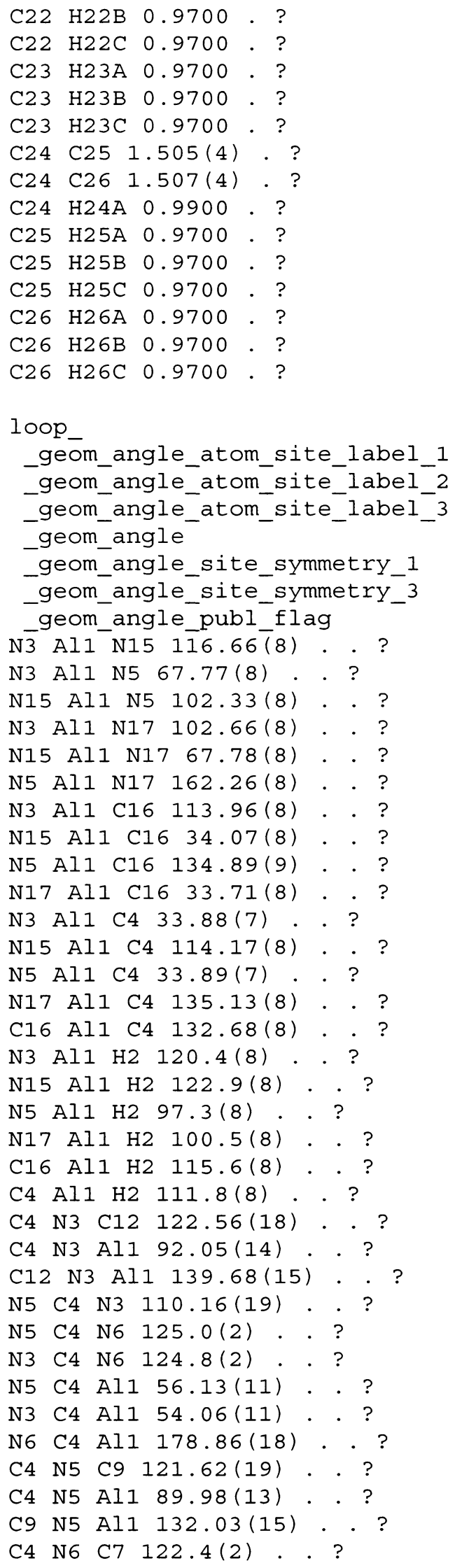




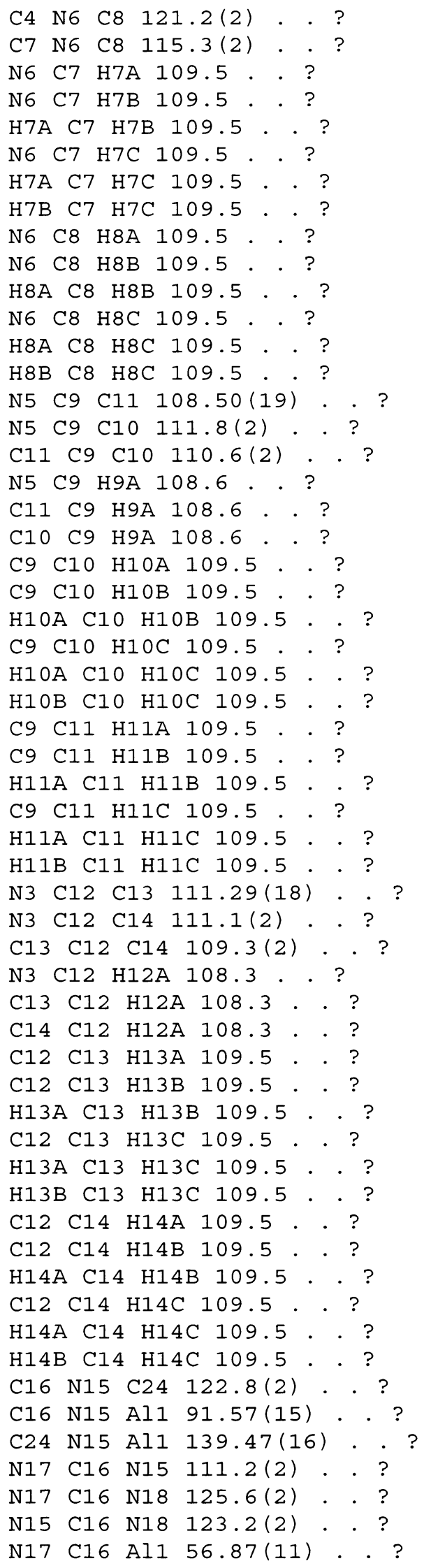




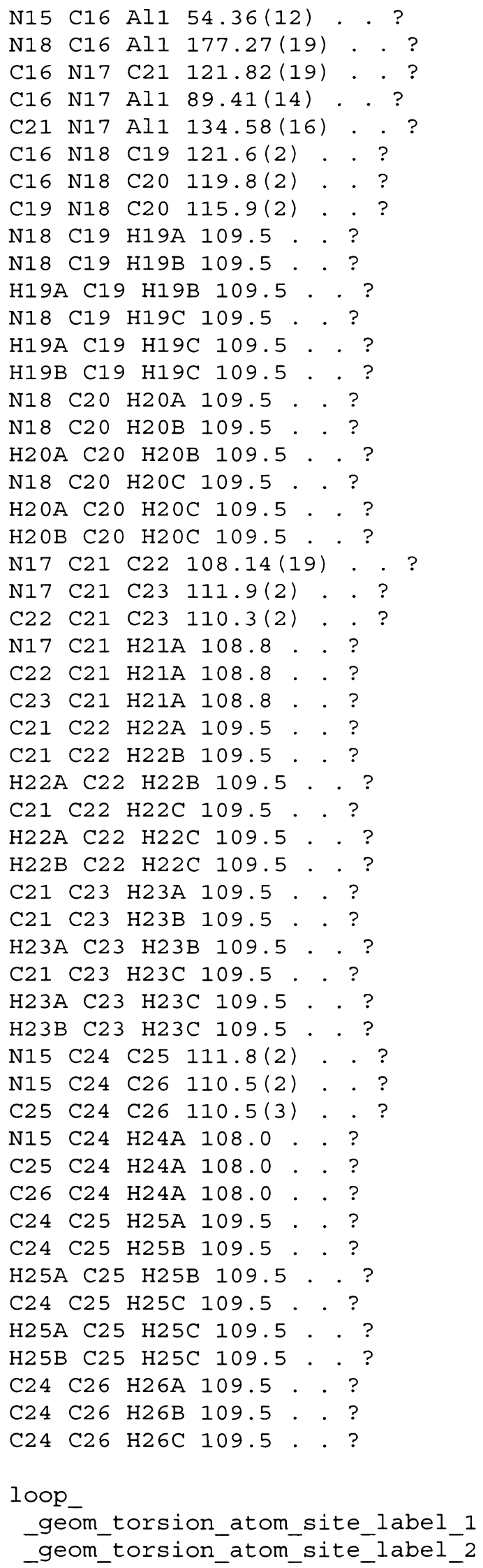




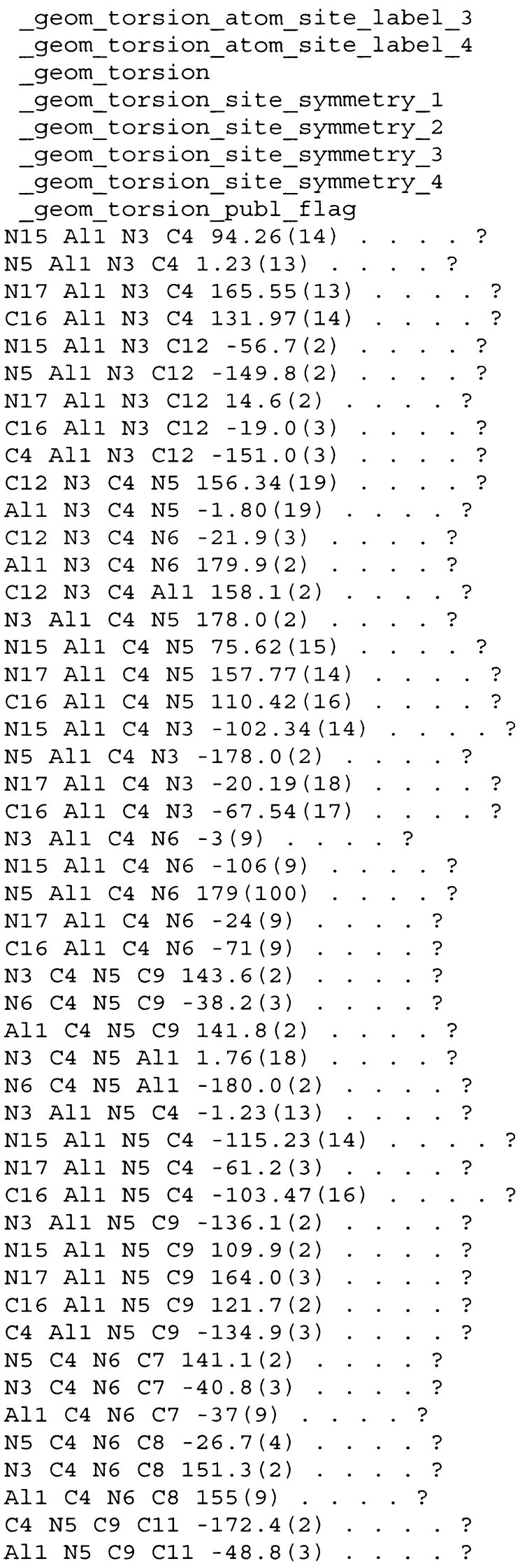




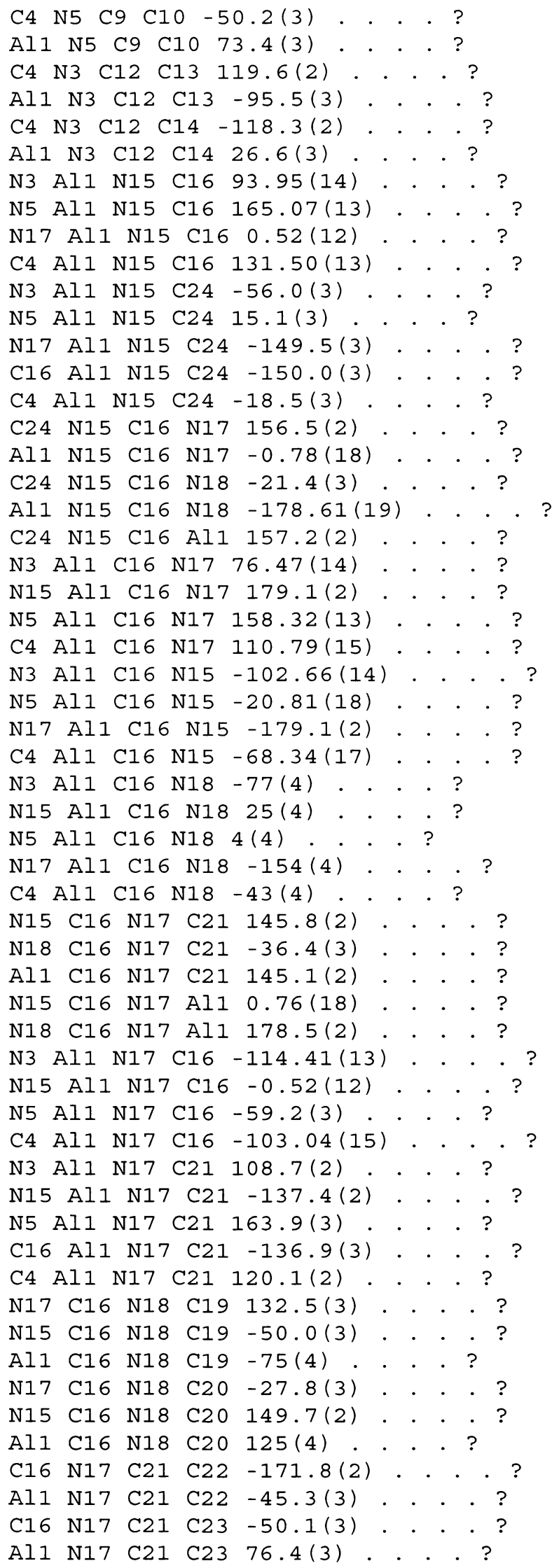


C16 N15 C24 C25 $-96.4(3)$. . . ?

A11 N15 $\mathrm{C} 24 \quad \mathrm{C} 25 \quad 47.1(4) \quad . \quad . \quad$ ?

C16 N15 C24 C26 $140.1(3)$. . . . ?

Al1 N15 C24 C26 $\quad$ C $76.4(3) \quad . \quad . \quad . \quad$ ?

_diffrn_measured_fraction_theta_max 0.925 _diffrn_reflns_theta_full 28.31

_diffrn_measured_fraction_theta_full 0.925

_refine_diff_density_max $0.2 \overline{4} 8$

refine_diff_density_min $\quad-0.345$

_refine_diff_density_rms 0.052 


\section{Compound 2: $\left[\mathrm{Et}_{2} \mathrm{NC}\left(\mathrm{N}^{\mathrm{i}} \mathrm{Pr}\right)_{2}\right]_{2} \mathrm{AlH}$}

View perpendicular to $\mathrm{Al}-\mathrm{H} 1$ bond

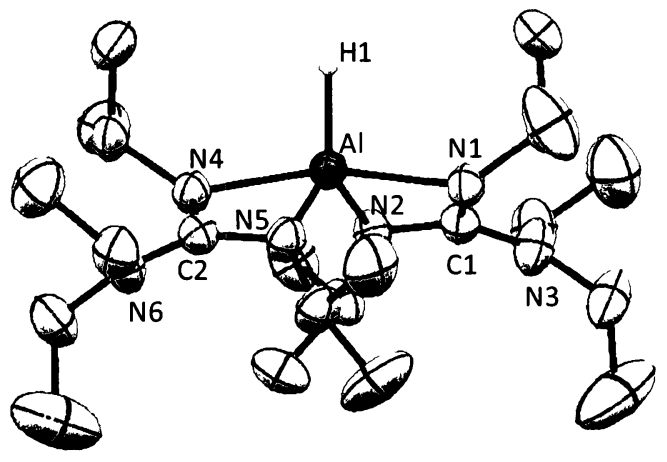

View along Al-H1 bond

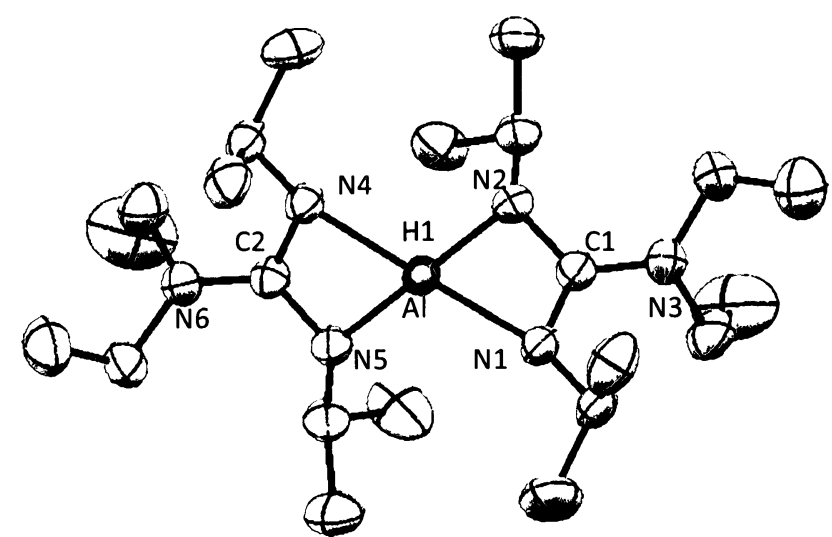

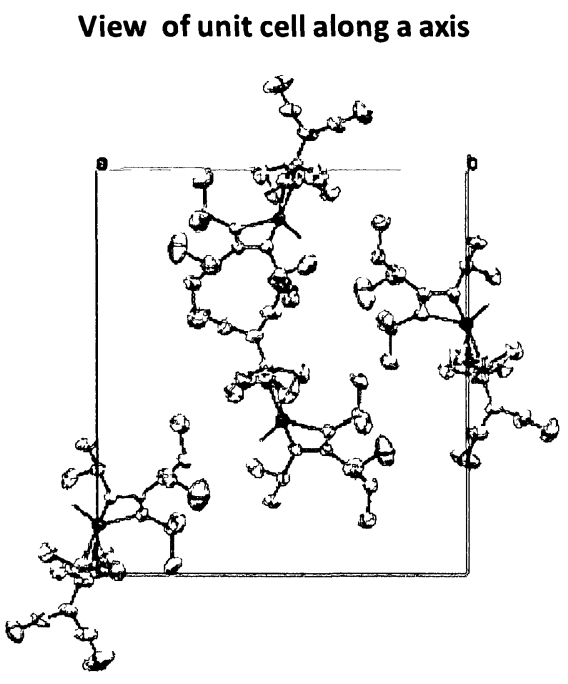

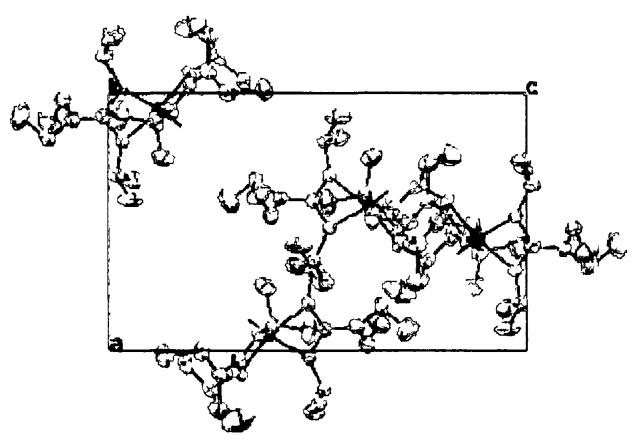

View of unit cell along $c$ axis

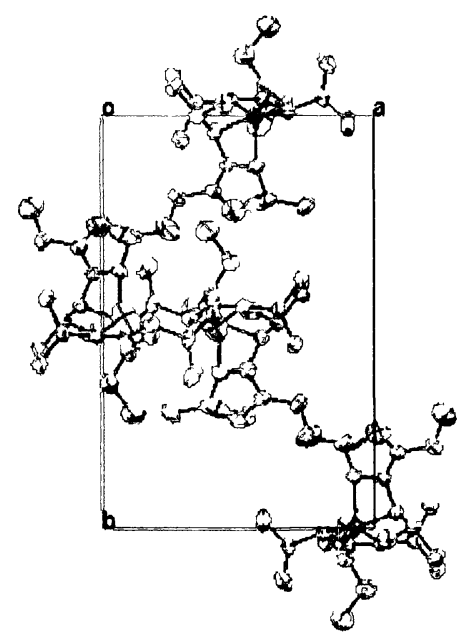




\section{Compound 2: $\left[\mathrm{Et}_{2} \mathrm{NC}\left(\mathrm{N}^{\mathrm{i} P r}\right)_{2}\right]_{2} \mathrm{AlH}$.cif file}

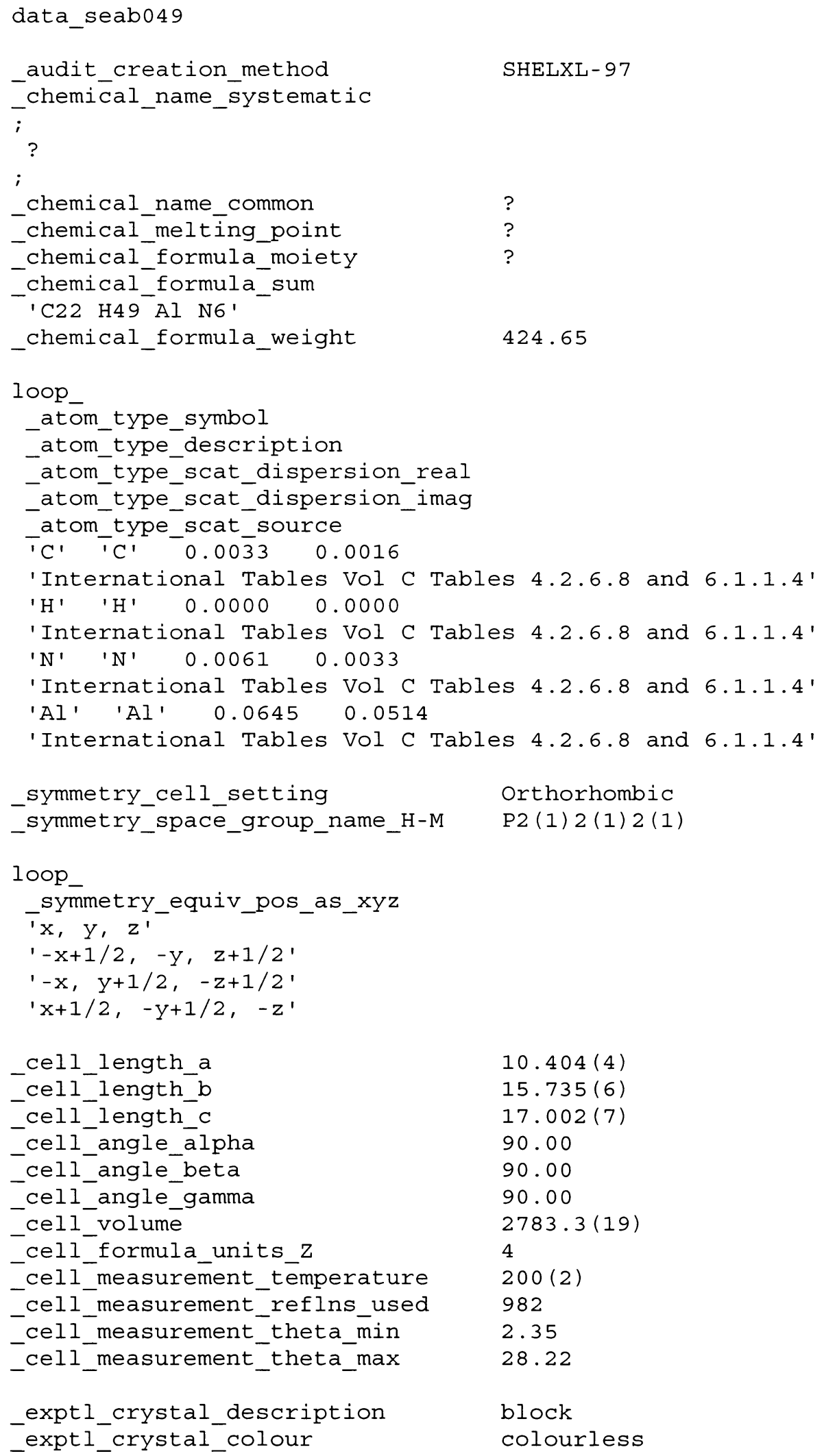




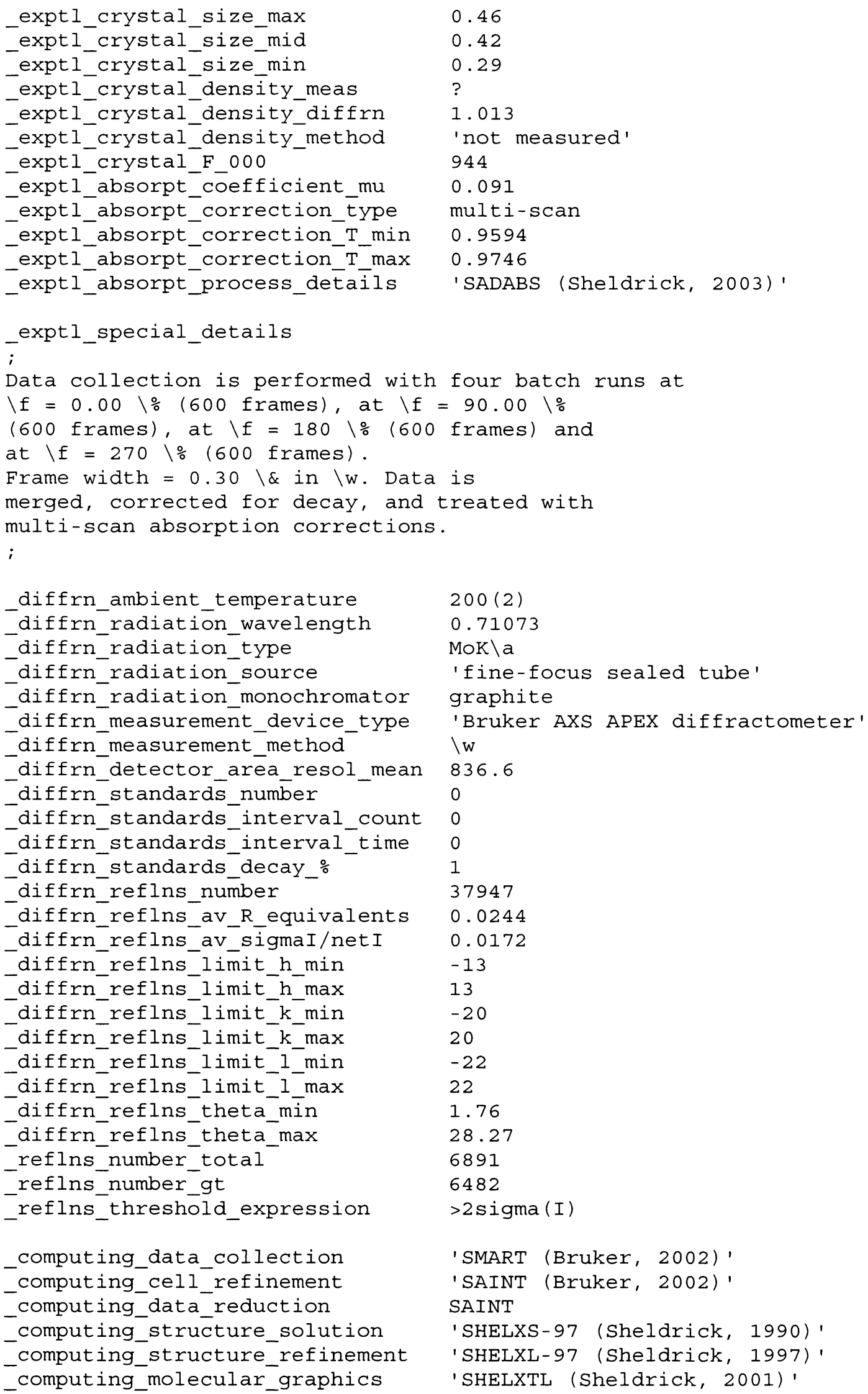


_computing_publication_material_SHELXTL (Sheldrick, 2001)'

_refine_special_details

;

Refinement of $\mathrm{F}^{\wedge} 2^{\wedge}$ against $\mathrm{ALL}$ reflections. The weighted $\mathrm{R}$-factor wR and goodness of fit $S$ are based on $F^{\wedge} 2^{\wedge}$, conventional $R$-factors $R$ are based on $F$, with $F$ set to zero for negative $F^{\wedge} 2^{\wedge}$. The threshold expression of $\mathrm{F}^{\wedge} 2^{\wedge}>2$ sigma $\left(\mathrm{F}^{\wedge} 2^{\wedge}\right)$ is used only for calculating $\mathrm{R}$-factors (gt) etc. and is not relevant to the choice of reflections for refinement. R-factors based on $F^{\wedge} 2^{\wedge}$ are statistically about twice as large as those based on $F$, and Rfactors based on ALL data will be even larger.

;

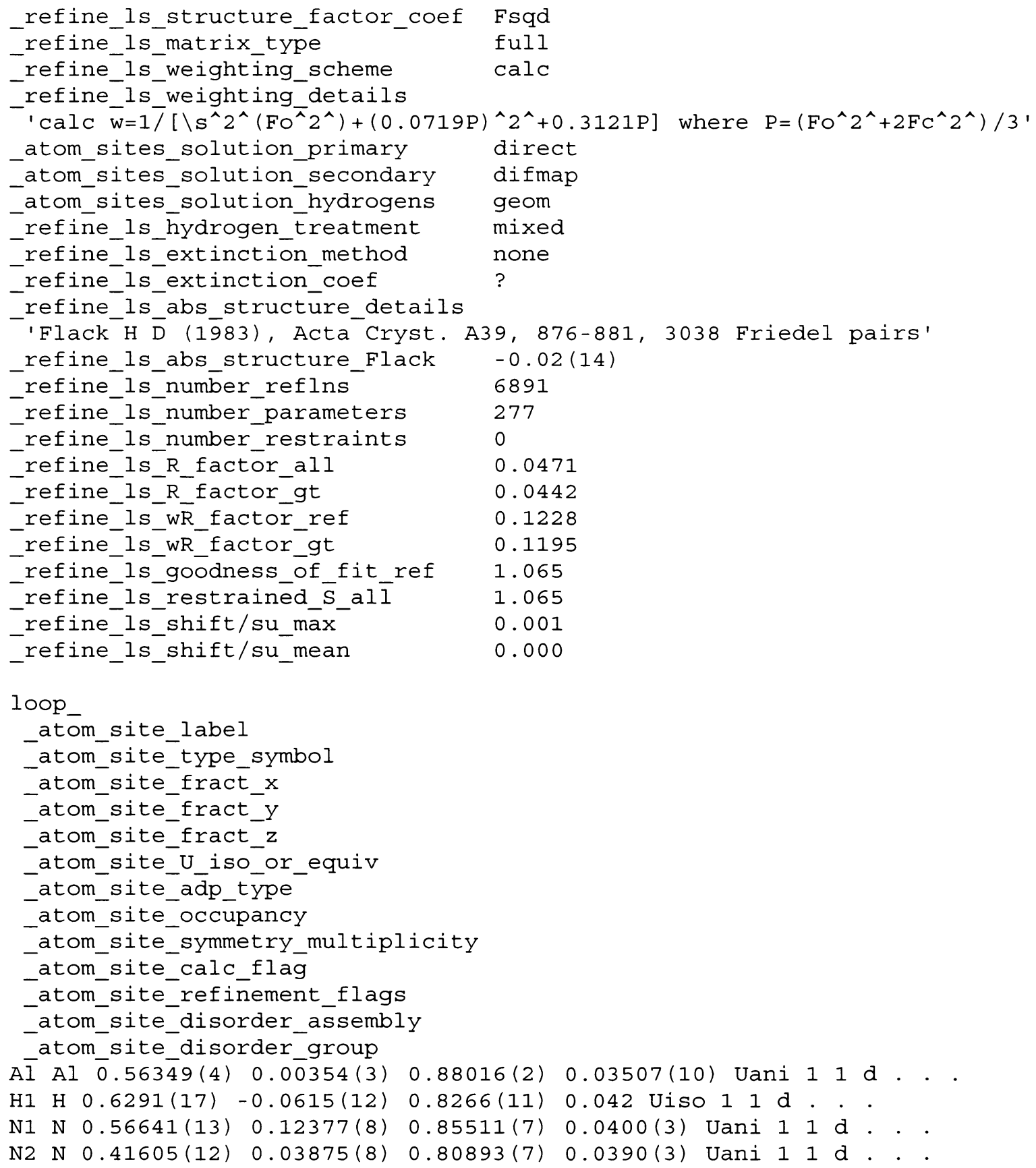


N3 N $0.41353(14) \quad 0.18276(9) \quad 0.76331(9) \quad 0.0480(3)$ Uani 11 d. . . N4 N $0.47682(11) \quad-0.03680(8) \quad 0.97392(7) \quad 0.0388(3) \quad$ Uani $11 d . .$. N5 N $0.68496(11) \quad-0.00954(9) \quad 0.97301(7) \quad 0.0385(3)$ Uani $11 d$. N6 N $0.59801(15) \quad-0.07238(11) \quad 1.09027(8) \quad 0.0526(4)$ Uani $11 d$. C1 C $0.60858(18) \quad 0.20542(10) 0.88890(10) 0.0498(4)$ Uani 11 d . . H1A H $0.5593 \quad 0.25210 .86270 .060$ Uiso 11 Calc R. . C2 C $0.5813(4) \quad 0.20888(16) \quad 0.97653(13) \quad 0.0901(9)$ Uani 11 d . . H2A H 0.48830 .20630 .98540 .135 Uiso 11 calc R . .

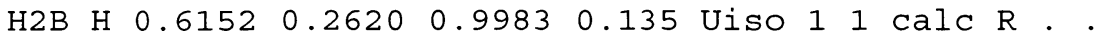
H2C H 0.62280 .16061 .00260 .135 Uiso 11 Calc $R$. C3 C $0.7505(2) \quad 0.21974(16) \quad 0.87359(16) \quad 0.0729(6)$ Uani 11 d. . . H3A H $0.76730 .21610 .8170 \quad 0.109$ Uiso 11 Calc R . . H3B H $0.8007 \quad 0.17620 .90110 .109$ Uiso 11 calc R. . H3C H $0.7752 \quad 0.27610 .89280 .109$ Uiso 1 l calc R. . C4 C $0.35346(16) \quad 0.00281(11) \quad 0.73860(10) \quad 0.0479(3)$ Uani $11 d .$. H4A H 0.29910 .04790 .71390 .057 Uiso 11 calc $R$. C5 C $0.2668(2) \quad-0.07033(14) \quad 0.76369(15) \quad 0.0665(5)$ Uani 11 d. . . H5A H $0.2011-0.04920 .80010 .100$ Uiso 11 Calc R. . H5B H $0.3184-0.11410 .78990 .100$ Uiso 11 calc R. H5C H $0.2250-0.09480 .71720 .100$ Uiso 11 Calc R. . C6 C $0.4523(2) \quad-0.02680(14) \quad 0.67874(10) \quad 0.0591(5)$ Uani 11 d. .

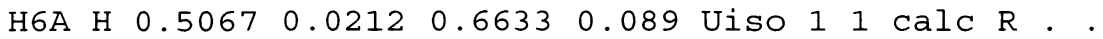

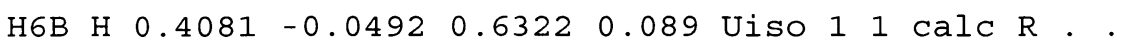
H6C H $0.5057-0.07150 .70200 .089$ Uiso 11 calc R. . C7 C $0.4966(2) \quad 0.23962(11) \quad 0.71799(10) \quad 0.0528(4)$ Uani 11 d . . H7A H $0.5871 \quad 0.2291 \quad 0.7331 \quad 0.063$ Uiso 11 Calc $R$. . H7B H $0.4758 \quad 0.29910 .73190 .063$ Uiso 11 CalC R. . C8 C $0.4837(3) \quad 0.22887(16) \quad 0.62975(13) \quad 0.0732(6)$ Uani 11 d . . H8A H $0.53770 .27090 .6030 \quad 0.110$ Uiso 11 Calc $R$. . $\begin{array}{lllllllllllll}\mathrm{H} 8 \mathrm{~B} & \mathrm{H} & 0.3937 & 0.2373 & 0.6144 & 0.110 & \text { Uiso } 1 & 1 & \mathrm{CalC} & \mathrm{R} & \text {. }\end{array}$ $\mathrm{H} 8 \mathrm{C}$ H $0.51110 .17150 .6148 \quad 0.110$ Uiso 1 l calc $\mathrm{R}$. . C9 C $0.27562(19) \quad 0.19780(13) \quad 0.76170(15) \quad 0.0620(5)$ Uani $11 d$. . H9A H $0.23120 .14810 .7850 \quad 0.074$ Uiso 11 calc R. . H9B H $0.2473 \quad 0.20290 .7063 \quad 0.074$ Uiso 1 l calc R. . C10 C $0.2361(4) \quad 0.2766$ (2) $0.8057(3) 0.1271$ (15) Uani 11 d. . HIOA H $0.1430 \quad 0.28460 .80080 .191$ Uiso 11 Calc $R$. . H10B H $0.2807 \quad 0.3260 \quad 0.78350 .191$ Uiso 11 Calc $R$. . H10C H $0.2588 \quad 0.2706 \quad 0.86130 .191$ Uiso 11 Calc R. . C11 C $0.46350(14) \quad 0.11727(9) \quad 0.80775(8) \quad 0.0378(3)$ Uani $11 d .$. C12 C $0.35079(15)-0.02961(11) 1.01245(10) 0.0457(3)$ Uani $11 d .$. H12A H $0.3639-0.03301 .07060 .055$ Uiso 11 calc R. . C13 C $0.28998(18) \quad 0.05630(13) \quad 0.99321(14) \quad 0.0600(5)$ Uani 11 d . . H13A H 0.34690 .10201 .01110 .090 Uiso 11 Calc R. . H13B H $0.20690 .06101 .0200 \quad 0.090$ Uiso 11 calc R. . $\mathrm{H} 13 \mathrm{C}$ H 0.27720 .06090 .93630 .090 Uiso 1 I Calc R. . C14 C $0.26110(18)-0.10133(14) \quad 0.98800(15) 0.0623(5)$ Uani $11 d$. . H14A H $0.3010-0.15611 .00050 .093$ Uiso 11 Calc R. . H14B H $0.2450-0.0980 \quad 0.93130 .093$ Uiso 11 Calc R. . $\mathrm{H} 14 \mathrm{C} \mathrm{H} 0.1796-0.09611 .01640 .093$ Uiso 11 calc $\mathrm{R}$. . C15 C $0.81582(15) \quad-0.04279(12) \quad 0.98449(10) \quad 0.0476(4)$ Uani $11 d$. H15A H $0.8318-0.04881 .04220 .057$ Uiso 11 calc R. . C16 C $0.91105(19) \quad 0.02031(19) \quad 0.9516(2) \quad 0.0903(9)$ Uani 11 d. . . H16A H $0.9078 \quad 0.0728 \quad 0.98250 .135$ Uiso 11 CalC R. . H16B H $0.88930 .0328 \quad 0.89670 .135$ Uiso 11 Calc $R$. . $\mathrm{H} 16 \mathrm{C} \mathrm{H} 0.9978-0.00380 .95420 .135$ Uiso $11 \mathrm{CalC} R$. . C17 C $0.8327(2) \quad-0.12913(16) \quad 0.94621(14) \quad 0.0689(6)$ Uani $11 d .$. H17A H $0.7687-0.16870 .96730 .103$ Uiso 11 calc R . . 
H17B H $0.9192 \quad-0.1507 \quad 0.9573 \quad 0.103$ Uiso 11 calc $\mathrm{R}$. .

H17C H $0.8212-0.12380 .88920 .103$ Uiso 11 calc $R$. .

C18 C $0.5268(2) \quad-0.14871(14) \quad 1.11350(12) \quad 0.0614(5)$ Uani $11 d .$.

H18A H $0.4776-0.16981 .06770 .074$ Uiso 11 calc $R$. .

H18B H $0.4644-0.13341 .15510 .074$ Uiso 11 calc $R$. .

C19 C $0.6129(3) \quad-0.21946(19) \quad 1.14360(19) \quad 0.0919(9)$ Uani $11 d .$.

H19A H $0.5605-0.26941 .15610 .138$ Uiso 11 Calc $R$. .

H19B H $0.6578-0.20031 .19110 .138$ Uiso 11 calc $R$. .

H19C H $0.6760-0.23431 .10310 .138$ Uiso 11 calc $R$. .

C20 C $0.6708(2)-0.02671(17) \quad 1.14971(11) \quad 0.0645(5)$ Uani $11 d .$.

H20A H 0.71660 .02131 .12460 .077 Uiso 11 Calc R. .

H20B H $0.7361-0.06521 .17260 .077$ Uiso 11 Calc $R$.

C21 C $0.5865(4) \quad 0.0069(3) \quad 1.21444(18) \quad 0.1271(15)$ Uani $11 d .$.

H21A H 0.64020 .03151 .25590 .191 Uiso 11 calc R. .

H21B H $0.5350-0.03961 .23630 .191$ Uiso 11 calc $R$. .

H21C H 0.52920 .05071 .19320 .191 Uiso 11 Calc R. .

C22 C $0.58711(14)-0.03988(10) \quad 1.01513(8) 0.0393(3)$ Uani 11 d. .

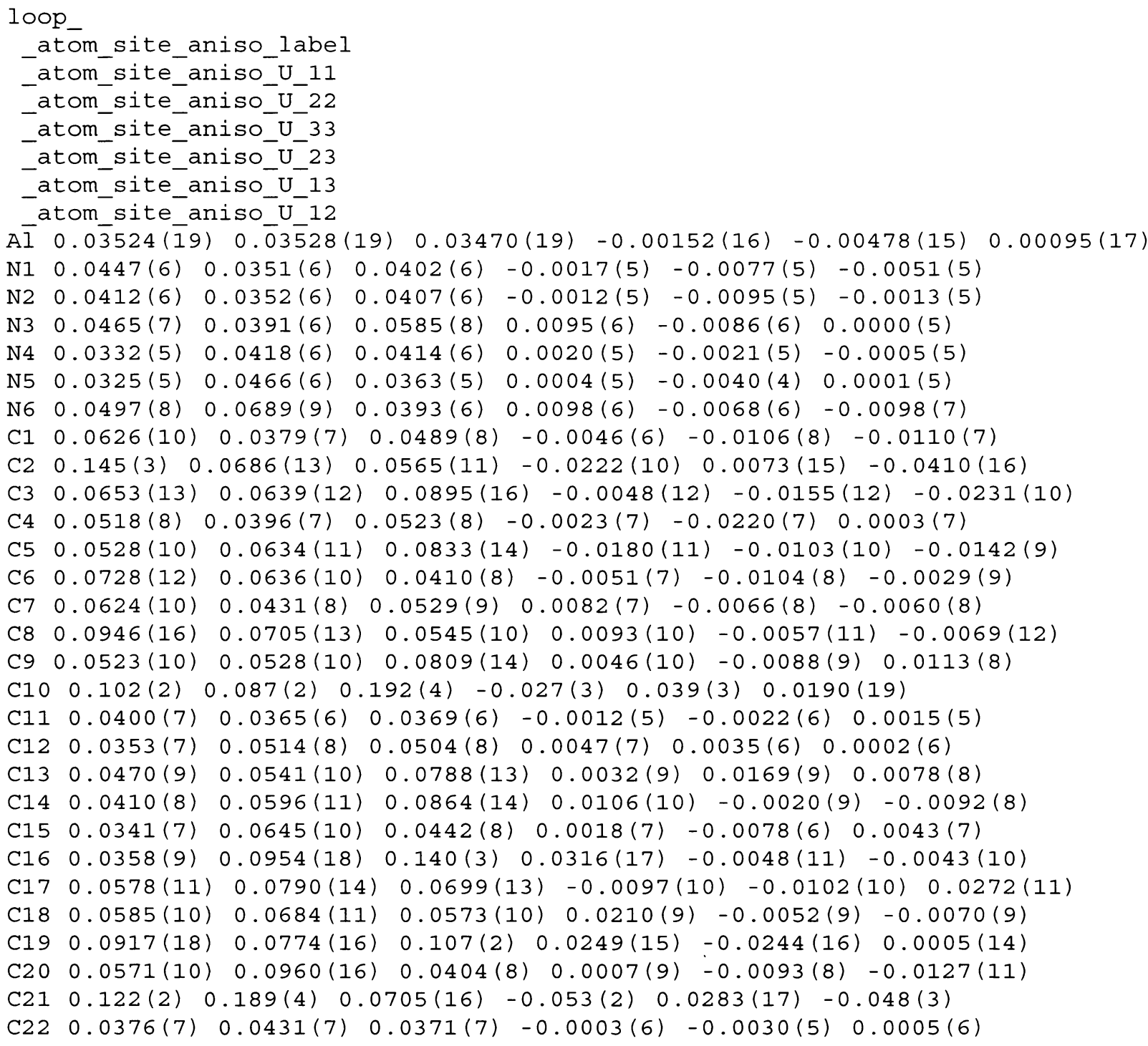

_geom_special_details 
All esds (except the esd in the dihedral angle between two l.s. planes) are estimated using the full covariance matrix. The cell esds are taken into account individually in the estimation of esds in distances, angles and torsion angles; correlations between esds in cell parameters are only used when they are defined by crystal symmetry. An approximate (isotropic) treatment of cell esds is used for estimating esds involving l.s. planes.

;

loop

_geom_bond_atom_site_label_1

-geom_bond_atom_site_label_2

-geom_bond_distance

_geom_bond_site_symmetry_2

-geom_bond_publ_flag

Ā N4 $\overline{1} .9384(14)$. ?

Al N1 1.9395(15). ?

Al N2 2.0314(14). ?

Al N5 2.0326 (13). ?

Al C22 2.4069(17).?

Al C11 2.4083(16). ?

N1 C11 1.3436(19) . ?

N1 C1 1.4741(19) . ?

N2 C11 $1.3306(19)$. ?

N2 C4 1.4743 (19) . ?

N3 C11 1.3795(19) . ?

N3 C9 $1.454(2)$. ?

N3 C7 $1.463(2)$. ?

N4 C22 1.3453(19) . ?

N4 C12 $1.470(2)$. ?

N5 C22 1.333(2) . ?

N5 C15 1.4715(19) . ?

N6 C22 1.381(2).?

N6 C20 1.453(3). ?

N6 C18 1.465(3) . ?

C1 C3 $1.516(3)$. ?

C1 C2 1.518(3).?

C4 C6 $1.520(3)$. ?

C4 C5 $1.523(3)$. ?

C7 C8 $1.516(3)$. ?

C9 C10 1.505(4) . ?

$\mathrm{C} 12 \mathrm{C} 14$ 1.522(3) . ?

$\mathrm{C} 12 \mathrm{C} 13 \mathrm{1.528}(3)$. ?

C15 C16 1.510(3). ?

C15 $\mathrm{C} 17 \quad 1.517(3)$. ?

C18 C19 $1.518(3)$. ?

C20 C21 1.504(4). ?

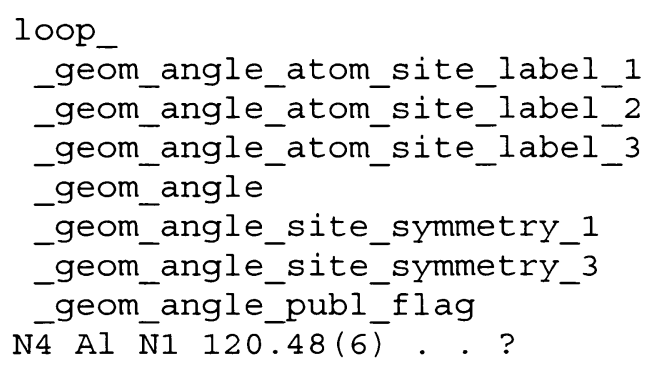




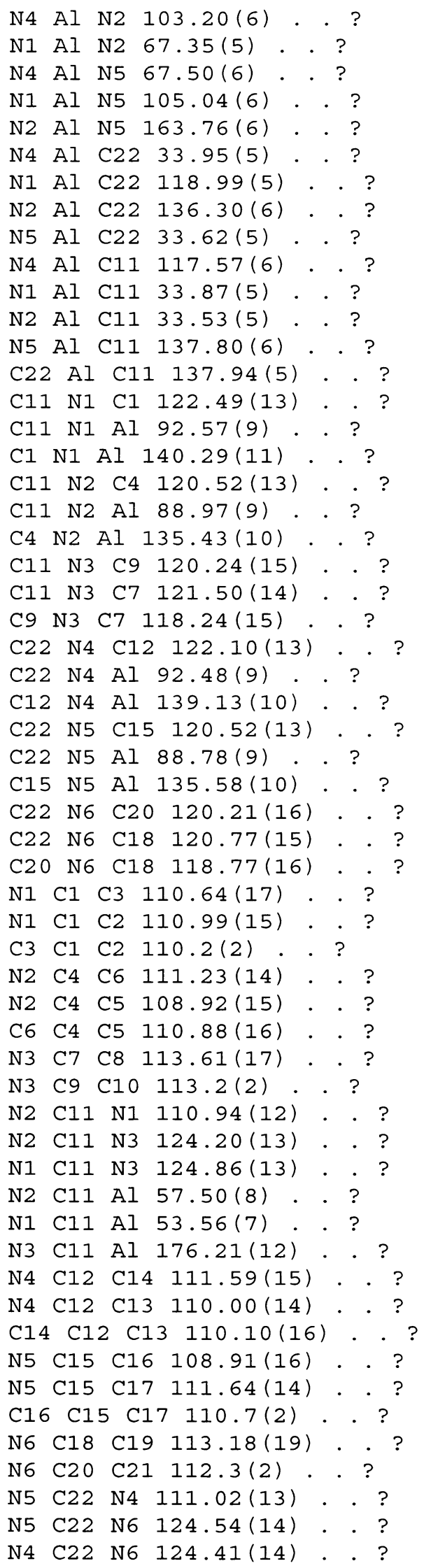




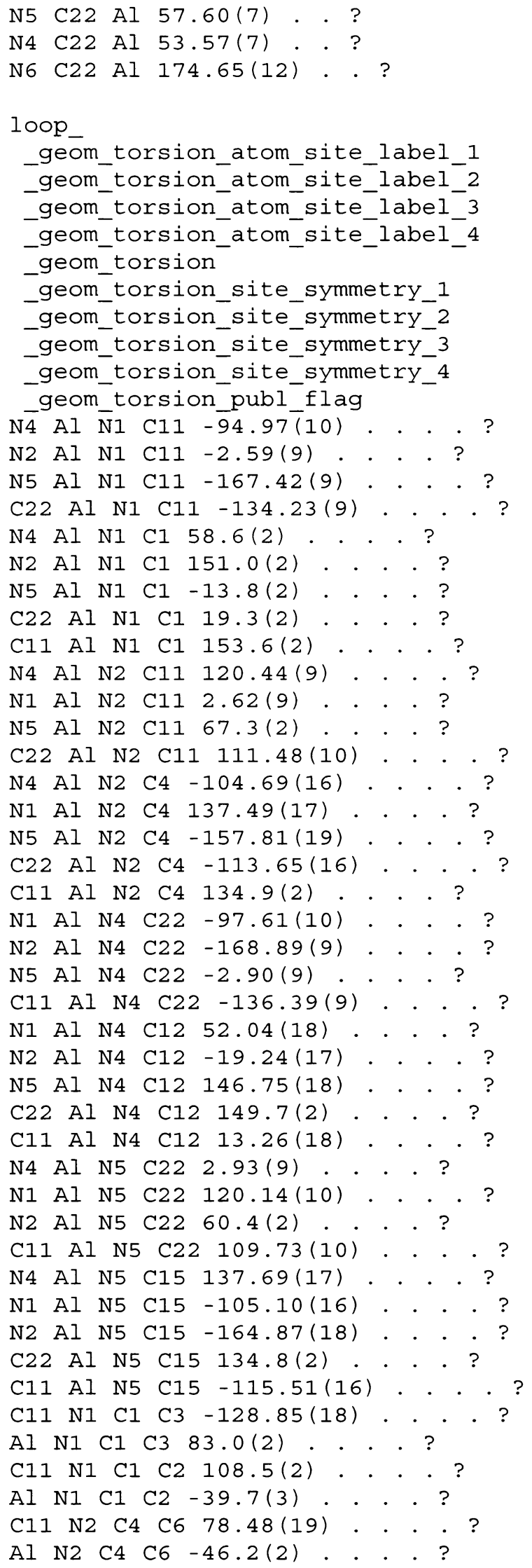




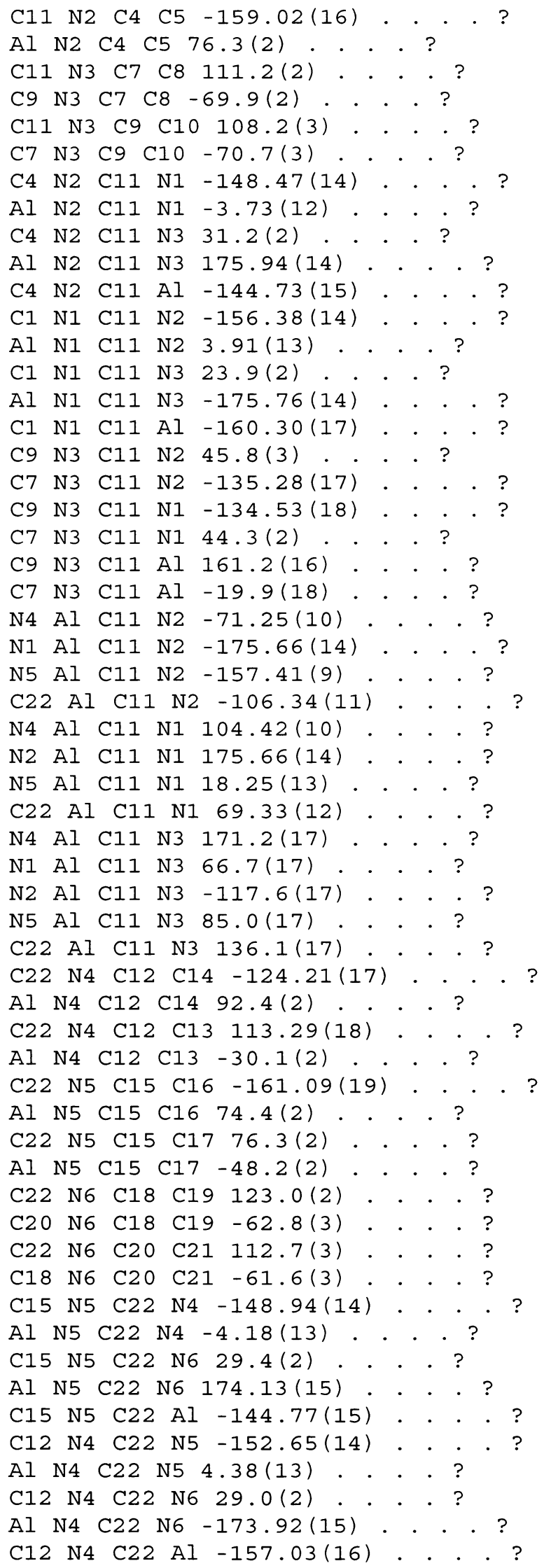




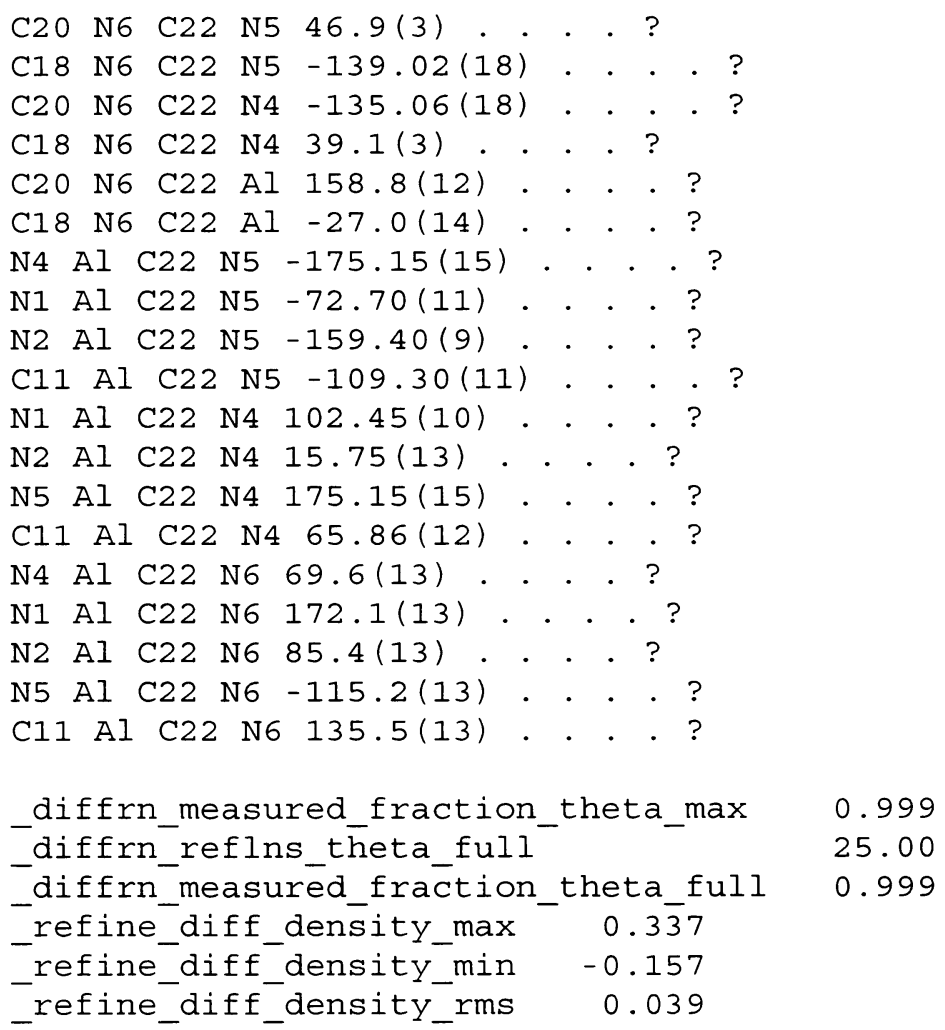




\section{Compound 4: [ $\left.{ }^{\mathrm{i}{ }_{2}} \mathrm{NC}\left(\mathrm{N}^{\mathrm{i}} \mathrm{Pr}\right)_{2}\right]_{2} \mathrm{AlH}$}

View perpendicular to $\mathrm{Al}-\mathrm{H} 1$ bond

View along Al-H1 bond
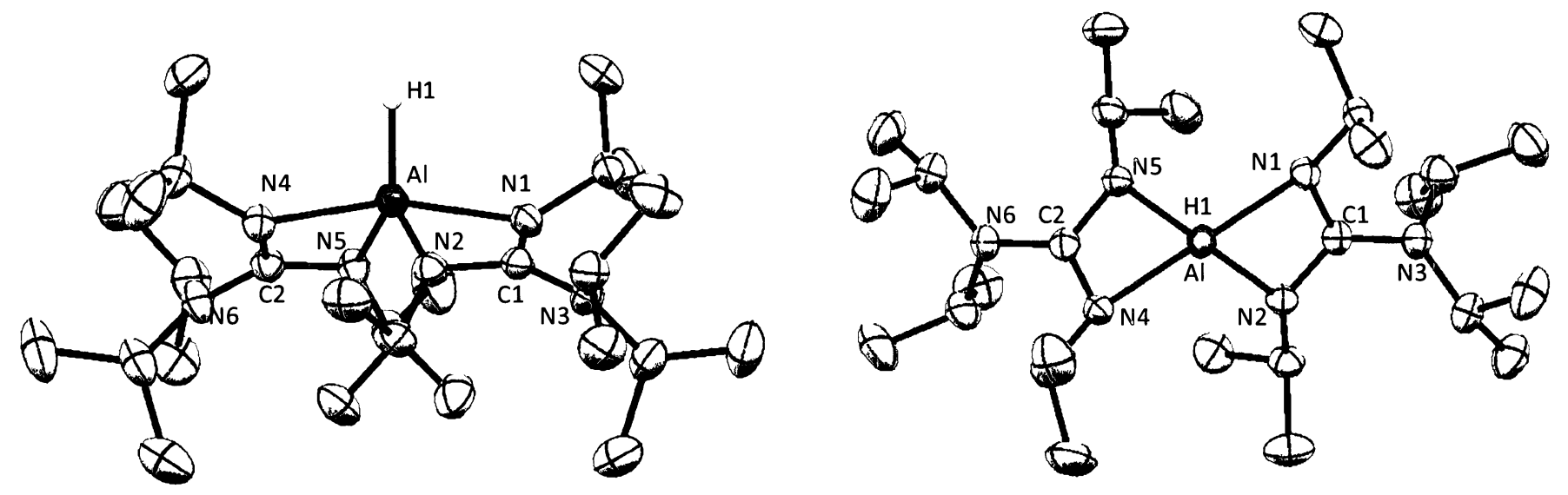

View of unit cell along a axis

View of unit cell along $b$ axis
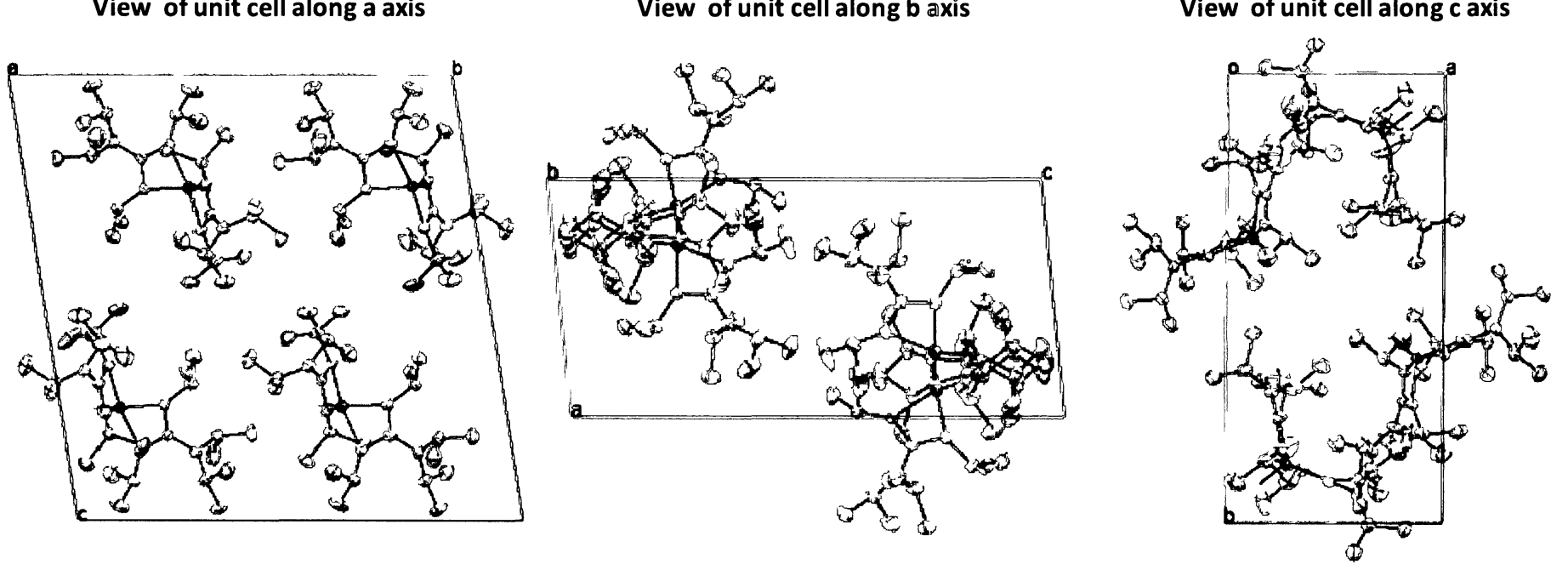


\section{Compound 4: [ $\left.\mathrm{Pr}_{2} \mathrm{NC}\left(\mathrm{N}^{\mathrm{i}} \mathrm{Pr}\right)_{2}\right]_{2} \mathrm{AlH}$.cif file}

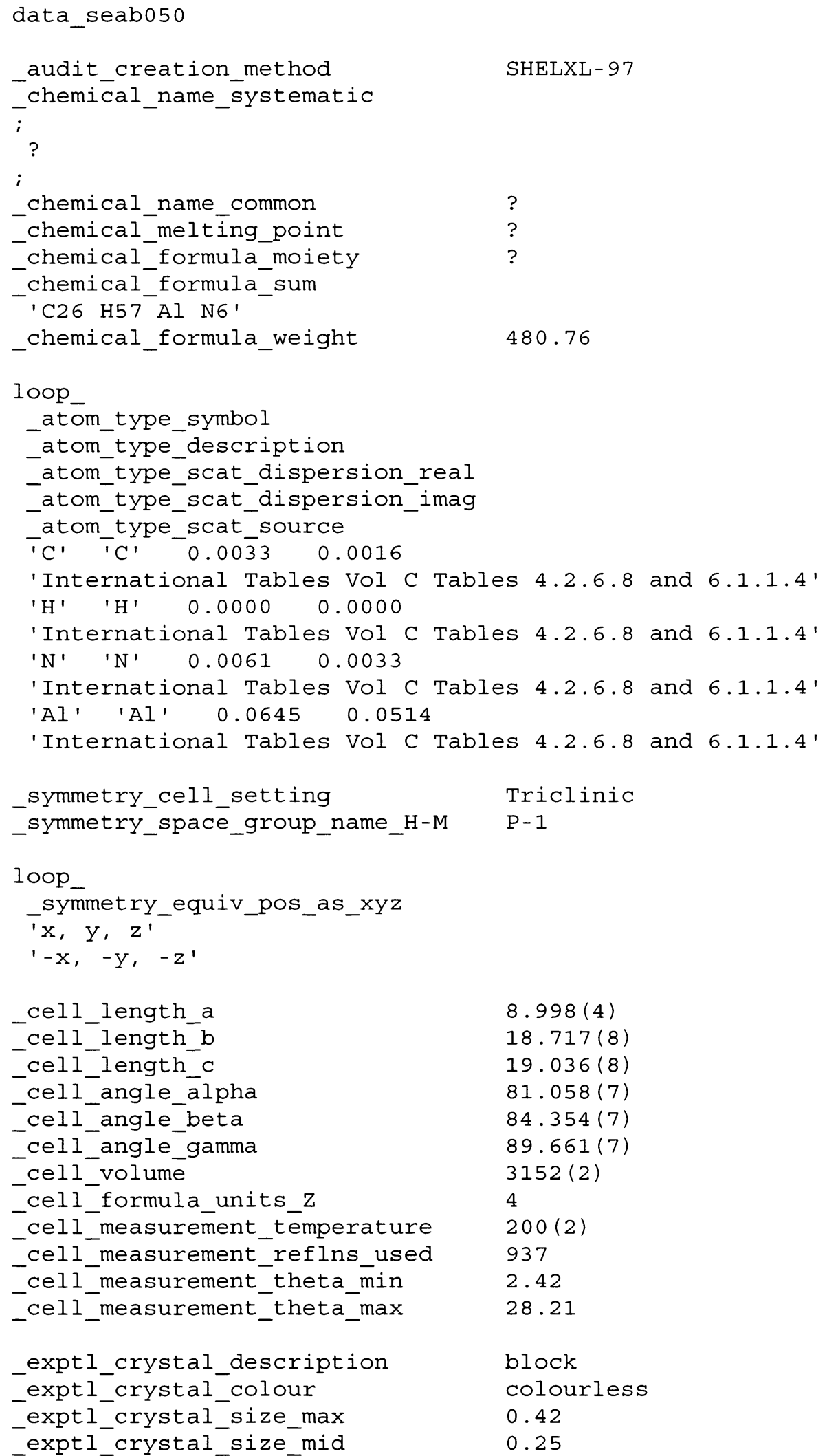




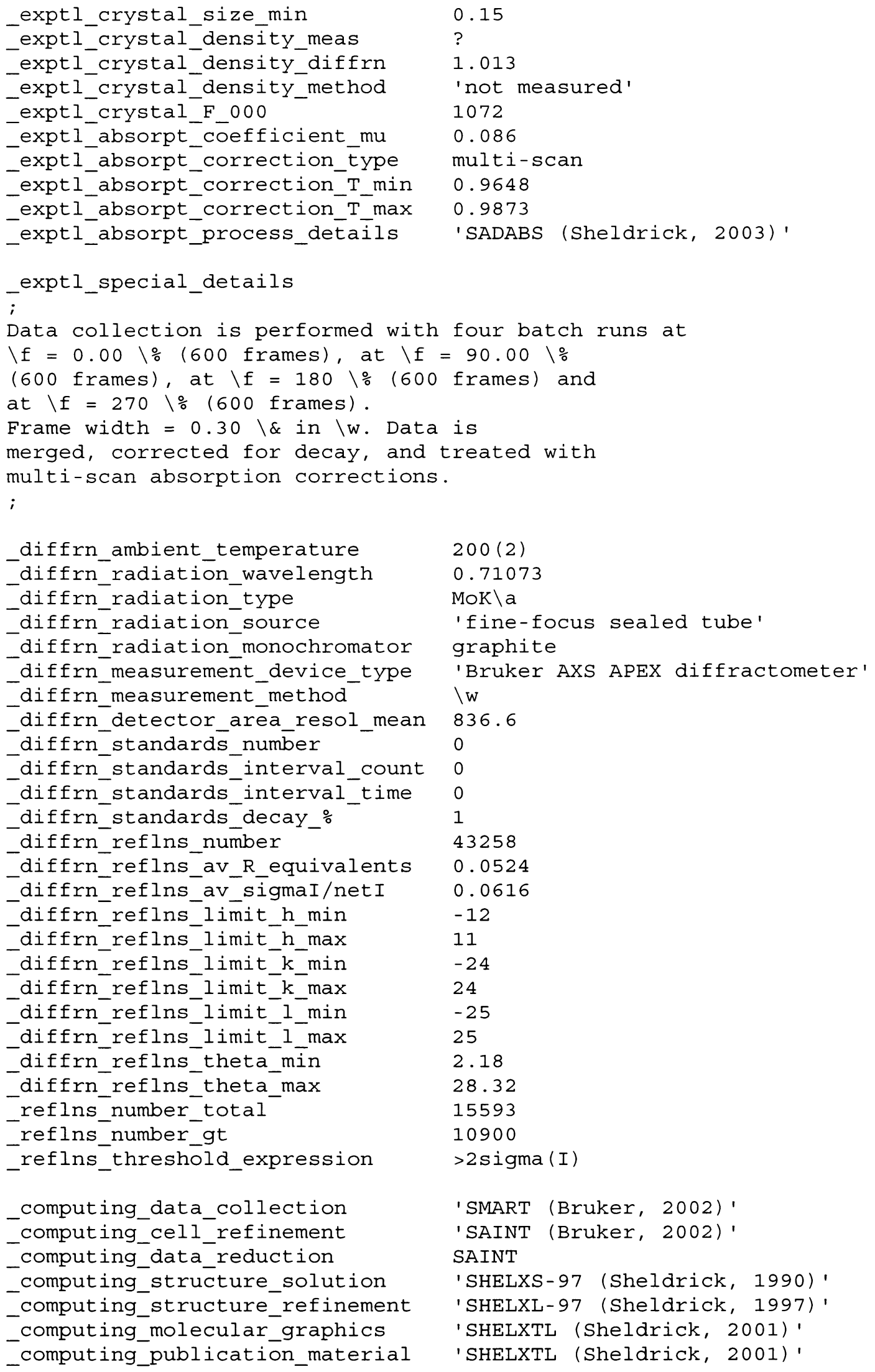




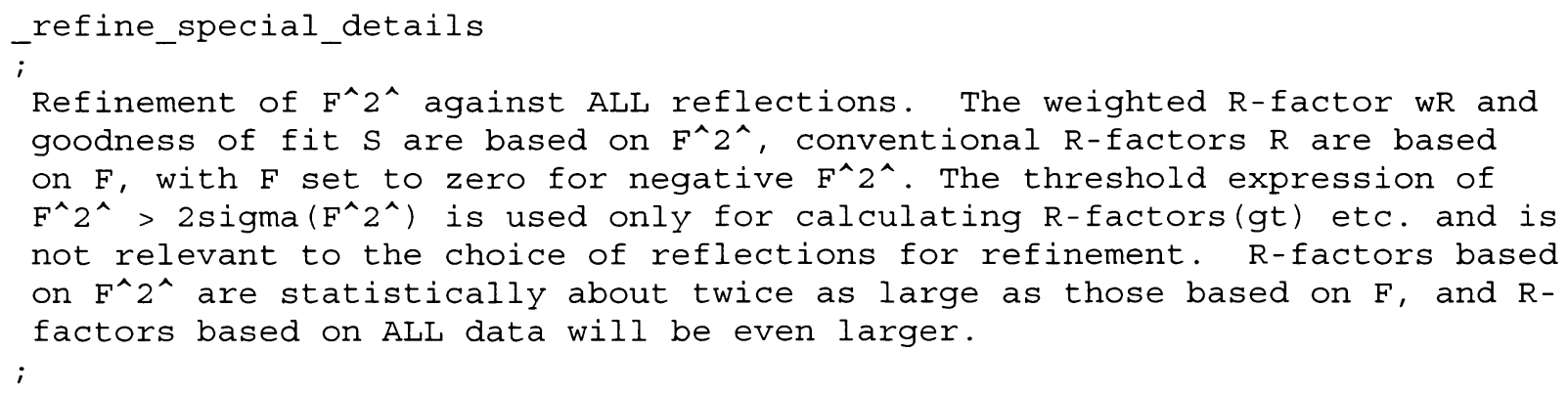

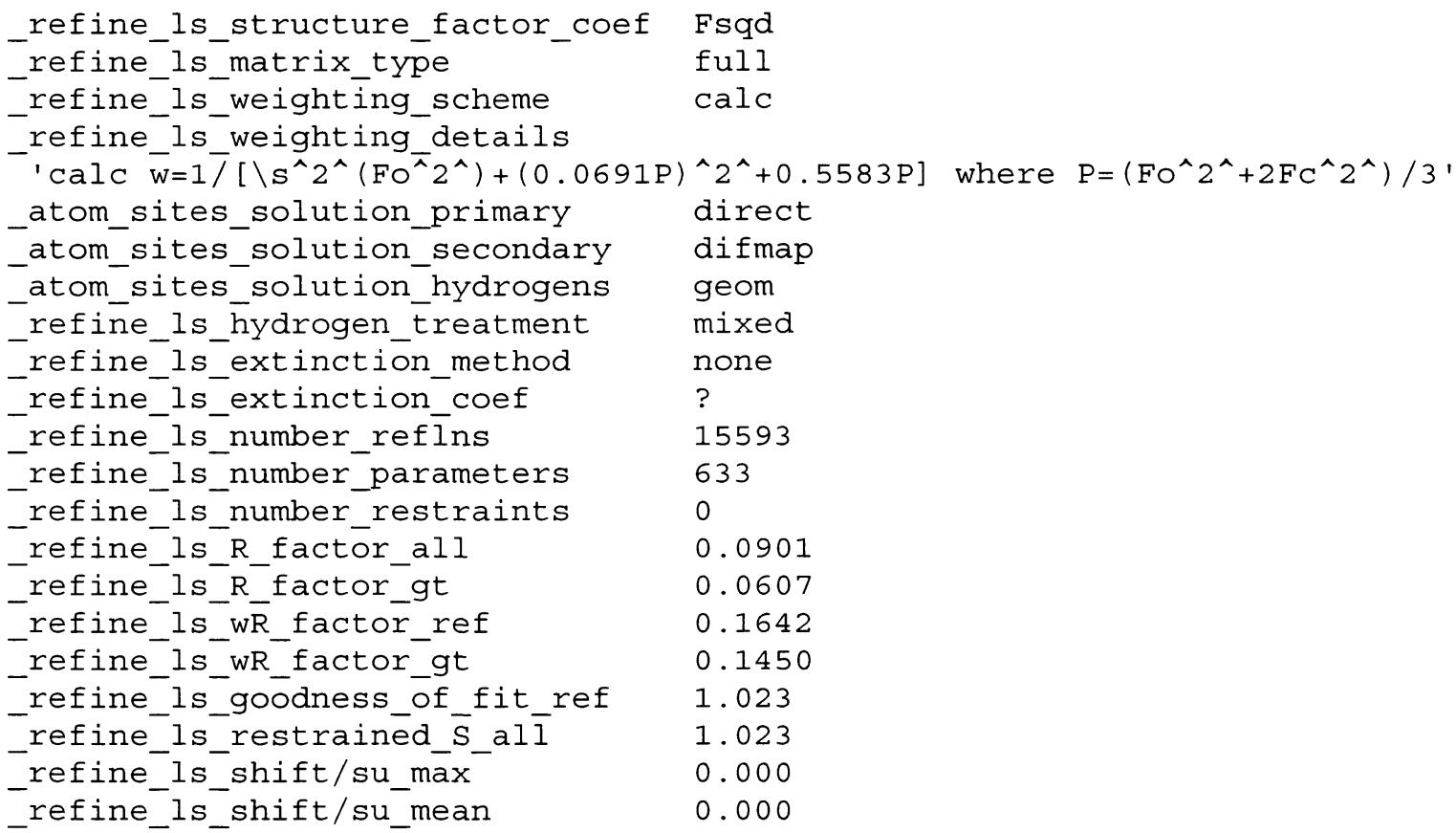

loop

atom site label

atom site type symbol

-atom_site_fract_x

-atom_site_fract_y

-atom_site_fract_z

-atom_site_U_iso_or_equiv

-atom_site_adp_type

-atom_site_occupancy

-atom_site_symmetry multiplicity

-atom_site_calc_flag

_atom_site_refiñement_flags

_atom_site_disorder_assembly

atom_site_disorder_group

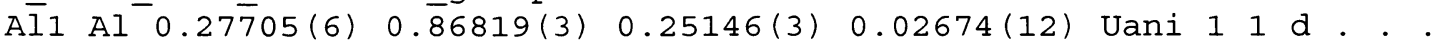

H1 H $0.143(2) 0.9168(9) \quad 0.2543(9) \quad 0.032$ Uiso 11 d. . .

Al2 Al $1.11429(5) \quad 0.36756(3) \quad 0.25543(3) \quad 0.02617(12)$ Uani $11 d .$.

$\mathrm{H} 2 \mathrm{H} 1.245(2) 0.4154(9) \quad 0.2609(9) 0.031$ Uiso $11 \mathrm{~d} .$.

N1 N $0.35811(16) \quad 0.87985(8) \quad 0.34630(7) \quad 0.0320(3)$ Uani $11 d . .$.

$\mathrm{N} 2 \mathrm{~N} \quad 0.47972(15) \quad 0.90422(8) \quad 0.23891(7) \quad 0.0287(3)$ Uani $11 \mathrm{~d} . .$.

N3 N $0.60527(16) 0.93259(8) \quad 0.33799(8) 0.0346(3)$ Uani $11 d . .$.

N4 N $0.25428(16) \quad 0.83658(7) \quad 0.15580(7) \quad 0.0293(3)$ Uani $11 d . .$.

N5 N $0.24759(16) 0.76523(7) \quad 0.25924(7) \quad 0.0292(3)$ Uani $11 d . .$. 
$\mathrm{N} 6 \mathrm{~N} \quad 0.20930(18) \quad 0.70946(8) \quad 0.15561(8) \quad 0.0358(3)$ Uani $11 d . .$. N7 N $0.99693(15) \quad 0.37725(8) \quad 0.35091(7) \quad 0.0279(3)$ Uani 11 d . . N8 N $0.91494(15) \quad 0.40431(8) \quad 0.24477(7) \quad 0.0279(3)$ Uani $11 d . .$. N9 N $0.75110(15) \quad 0.42935(8) \quad 0.34694(8) \quad 0.0312(3)$ Uani $11 d . .$. N10 N $1.17465(16) \quad 0.33902(7) \quad 0.15781(7) \quad 0.0286(3) \quad$ Uani $11 d .$. N11 N $1.14058(16) \quad 0.26470(7) \quad 0.25966(7) \quad 0.0289(3) \quad$ Uani $11 d .$. N12 N $1.22033(18) \quad 0.21244(8) \quad 0.15345(8) \quad 0.0343(3)$ Uani 11 d . . C1 C $0.3073(2) \quad 0.90197(12) \quad 0.41518(10) \quad 0.0453(5)$ Uani 11 d . . HIA H $0.3918 \quad 0.92790 .43090 .054$ Uiso 11 calc $R$. C2 C $0.2664(5) \quad 0.83696(18) \quad 0.47130(14) \quad 0.1096(14)$ Uani 11 d . . . H2A H $0.3494 \quad 0.8028 \quad 0.4726 \quad 0.164$ Uiso 11 calc $R$. H2B H $0.17710 .81340 .4596 \quad 0.164$ Uiso 11 calc $R$. . $\mathrm{H} 2 \mathrm{C} \mathrm{H} 0.24630 .85250 .51810 .164$ Uiso 11 calc $\mathrm{R}$. C3 C $0.1779(3) \quad 0.95445(18) \quad 0.40674(15) \quad 0.0822(10)$ Uani 11 d . . H3A H 0.20930 .99640 .37090 .123 Uiso 11 calc $R$. H3B H 0.14770 .97050 .45260 .123 Uiso 11 calc $R$. $\mathrm{H} 3 \mathrm{C} \mathrm{H} 0.09340 .93020 .39130 .123$ Uiso 11 calc $\mathrm{R}$. C4 C $0.6172(2) \quad 0.90174(10) \quad 0.19061(9) \quad 0.0324(4)$ Uani 11 d. . H4A H 0.70420 .90880 .21780 .039 Uiso 11 calc R . . C5 C $0.6216(2) \quad 0.96180(12) \quad 0.12654(11) \quad 0.0488(5)$ Uani 11 d . . H5A H $0.6171 \quad 1.00890 .14310 .073$ Uiso 11 Calc R . H5B H 0.53610 .95650 .09960 .073 Uiso 11 Calc $R$. . $\mathrm{H} 5 \mathrm{C}$ H $0.71450 .9588 \quad 0.09570 .073$ Uiso 11 Calc $\mathrm{R}$. C6 C $0.6323(2) \quad 0.82830(11) \quad 0.16598(11) \quad 0.0454(5)$ Uani $11 d . .$. H6A H $0.63560 .79050 .2076 \quad 0.068$ Uiso 11 Calc R. .

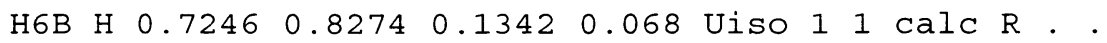
H6C H 0.54660 .81970 .14030 .068 Uiso 11 Calc $R$. . C7 C $0.6665(3) \quad 0.88504(13) \quad 0.39681(12) \quad 0.0557(6)$ Uani $11 d . .$. H7A H 0.58430 .85020 .41800 .067 Uiso 11 calc R . . C8 C $0.7937(3) \quad 0.83908(15) \quad 0.37016(16) \quad 0.0755(8)$ Uani 11 d . . $\begin{array}{lllllllllllll}\mathrm{H} 8 \mathrm{~A} & \mathrm{H} & 0.7602 & 0.8126 & 0.3340 & 0.113 & \text { Uiso } 1 & 1 & \mathrm{CalC} & \mathrm{R}\end{array}$.

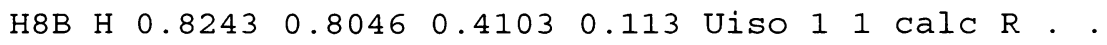

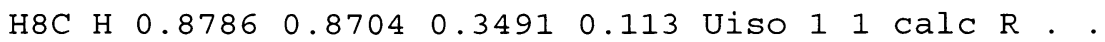
C9 C $0.7062(4) \quad 0.92323(18) \quad 0.45765(14) \quad 0.0840(9)$ Uani 11 d . . H9A H $0.61840 .9486 \quad 0.4757 \quad 0.126$ Uiso 11 calc $R$. H9B H 0.78720 .95820 .44010 .126 Uiso 11 calc $\mathrm{R}$. . $\mathrm{H} 9 \mathrm{C} \mathrm{H} 0.73870 .88750 .49630 .126$ Uiso $11 \mathrm{calc} R$. . C10 C $0.6611(2) 1.00528(10) \quad 0.30623(11) \quad 0.0405(4)$ Uani 11 d . . HIOA H 0.62101 .01560 .25860 .049 Uiso 11 Calc R. . C11 C $0.8306(2) \quad 1.01307(13) \quad 0.29079(14) \quad 0.0564(6)$ Uani 11 d . . H11A H $0.86820 .9766 \quad 0.26190 .085$ Uiso 11 Calc $R$. . H11B H 0.87641 .00610 .33590 .085 Uiso 11 Calc $R$. . H11C H 0.85581 .06150 .26470 .085 Uiso 11 Calc $R$. . C12 C $0.5964(3) \quad 1.06364(13) \quad 0.34807(14) \quad 0.0595(6)$ Uani $11 d$. . H12A H 0.48751 .05810 .35610 .089 Uiso 11 calc $R$. . H12B H 0.62201 .11150 .32080 .089 Uiso 11 calc R. . $\mathrm{H} 12 \mathrm{C} \mathrm{H} 0.63821 .05870 .39420 .089$ Uiso 11 calc $\mathrm{R}$. C13 C $0.48421(19) \quad 0.90589(9) \quad 0.30906(9) \quad 0.0291(4) \quad \operatorname{Uani} 11$ d . . C14 C $0.1834(2) \quad 0.86158(10) \quad 0.09024(10) \quad 0.0385(4)$ Uani $11 d$. . H14A H 0.14780 .81830 .07160 .046 Uiso 11 calc $R$. . C15 C $0.0496(3) \quad 0.90832(14) \quad 0.10595(14) \quad 0.0629(7)$ Uani $11 d . .$. H15A H -0.02130 .88080 .14250 .094 Uiso 11 calc $R$.

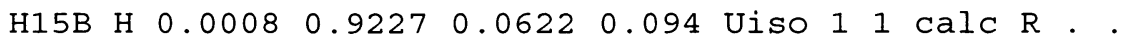
$\mathrm{H} 15 \mathrm{C} \mathrm{H} 0.08280 .9516 \quad 0.12330 .094$ Uiso 11 calc $\mathrm{R}$. . C16 C $0.2939(3) \quad 0.90339(15) \quad 0.03307(12) \quad 0.0655(7)$ Uani 11 d . . H16A H $0.38230 .87380 .0252 \quad 0.098$ Uiso 11 Calc R. . $\mathrm{H} 16 \mathrm{~B}$ H $0.3236 \quad 0.94820 .04880 .098$ Uiso 11 Calc $\mathrm{R}$. . 
H16C H $0.24690 .9151-0.01160 .098$ Uiso 1 l calc R. .

C17 C $0.2798(2) \quad 0.69838(10) 0.30622(9) \quad 0.0364(4)$ Uani $11 d$. . H17A H $0.28090 .65750 .2781 \quad 0.044$ Uiso 1 l calc R. .

C18 C $0.1580(3) \quad 0.68313(12) 0.36864(11) 0.0529(6)$ Uani $11 d . .$.

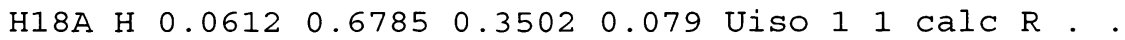

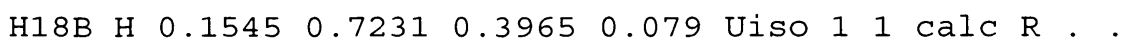
H18C H $0.1801 \quad 0.6380 \quad 0.3993 \quad 0.079$ Uiso 1 l CalC R . . C19 C $0.4319(2) \quad 0.70292(11) \quad 0.33378(11) \quad 0.0460(5)$ Uani $11 d . .$. H19A H $0.5097 \quad 0.7086 \quad 0.29340 .069$ Uiso 11 Calc R . .

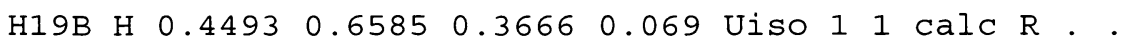

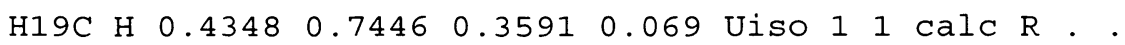
$\mathrm{C} 20 \mathrm{C} 0.0844(2) \quad 0.66003(10) \quad 0.18653(11) \quad 0.0428(5)$ Uani $11 \mathrm{~d} .$. $\mathrm{H} 20 \mathrm{~A} \mathrm{H} \quad 0.0575 \quad 0.6710 \quad 0.2360 \quad 0.051$ Uiso 1 l calc R. . C21 C $-0.0561(3) \quad 0.67513(13) \quad 0.14761(15) \quad 0.0639(7)$ Uani $11 d .$. H21A H $-0.0767 \quad 0.7271 \quad 0.14150 .096$ Uiso 11 calc R. . H21B H $-0.1410 \quad 0.64850 .1758 \quad 0.096$ Uiso 1 I calc R. . H21C H -0.04050 .65950 .10070 .096 Uiso 1 I calc R. . C22 C $0.1227(3) \quad 0.57979(11) \quad 0.19538(13) \quad 0.0533(6)$ Uani $11 d . .$.

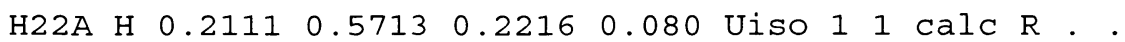
$\begin{array}{llllllllllllllll}\mathrm{H} 22 \mathrm{~B} & \mathrm{H} & 0.1431 & 0.5651 & 0.1482 & 0.080 & \text { Uiso } 1 & 1 & \mathrm{CalC} & \mathrm{R}\end{array}$. H22C H $0.0383 \quad 0.5514 \quad 0.2220 \quad 0.080$ Uiso 11 calc R . . C23 C $0.3133(3) \quad 0.70042(11) \quad 0.09272(10) 0.0460(5)$ Uani $11 d . .$. H23A H $0.3602 \quad 0.7490 \quad 0.0750 \quad 0.055$ Uiso 11 calc R . C24 C $0.4414(3) \quad 0.64947(13) \quad 0.11264(14) \quad 0.0636(7)$ Uani $11 d . .$. H24A H $0.4870 \quad 0.66450 .1528 \quad 0.095$ Uiso 1 l calc R. . $\begin{array}{lllllllllllll}\mathrm{H} 24 \mathrm{~B} & \mathrm{H} & 0.5166 & 0.6514 & 0.0715 & 0.095 & \text { Uiso } 1 \mathrm{calC} & \mathrm{R}\end{array}$. $\mathrm{H} 24 \mathrm{C} \mathrm{H} 0.4026 \quad 0.59990 .12640 .095$ Uiso 1 l calc R . . C25 C $0.2400(4) \quad 0.67889(15) \quad 0.03000(13) \quad 0.0743(8)$ Uani $11 d .$. H25A H 0.16120 .71340 .01700 .111 Uiso 11 calc R. . H25B H 0.19650 .63020 .04370 .111 Uiso 1 l calc R. . $\mathrm{H} 25 \mathrm{C} \mathrm{H} 0.31520 .6792-0.01100 .111$ Uiso 1 I calc R . . C26 C $0.23574(19) \quad 0.76867(9) \quad 0.18901(9) \quad 0.0284(3)$ Uani $11 d . .$. C27 C $1.0242(2) \quad 0.39900(10) 0.41947(9) \quad 0.0336(4)$ Uani $11 d . .$. H27A H $0.92640 .41140 .4442 \quad 0.040$ Uiso 1 l calc R . C28 C $1.0916(3) \quad 0.33662(12) \quad 0.46625(10) \quad 0.0500(5)$ Uani $11 d . .$.

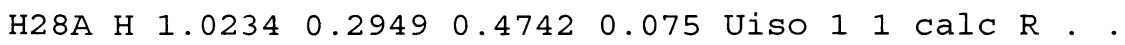
$\begin{array}{lllllllllllll}\mathrm{H} 28 \mathrm{~B} & \mathrm{H} & 1.1078 & 0.3511 & 0.5122 & 0.075 & \text { Uiso } 1 & 1 & \mathrm{CalC} & \mathrm{R}\end{array}$.

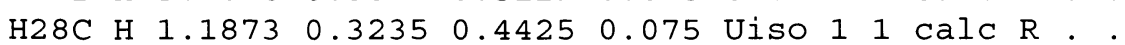
C29 C $1.1263(3) \quad 0.46507(12) \quad 0.40808(12) \quad 0.0509(5)$ Uani $11 d . .$. H29A H 1.08370 .50420 .37590 .076 Uiso 1 l calc R . . H29B H $1.22490 .4527 \quad 0.38690 .076$ Uiso 1 l calc R . . H29C H 1.13610 .48090 .45410 .076 Uiso 1 l calc R. . C30 C $0.7960(2) \quad 0.40335(10) \quad 0.19722(9) \quad 0.0323(4)$ Uani $11 d$. .

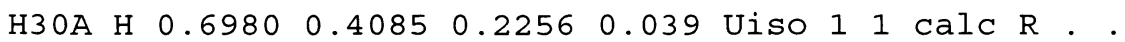
C31 C $0.8134(3) \quad 0.46616(12) 0.13531(11) \quad 0.0494(5)$ Uani $11 d . .$. H31A H $0.8082 \quad 0.5120 \quad 0.1542 \quad 0.074$ Uiso 1 l calc R. .

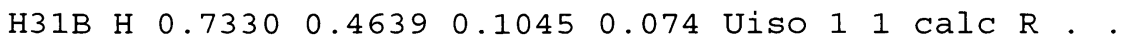
H31C H 0.91010 .46280 .10750 .074 Uiso 1 l calc R. . C32 C $0.7941(2) 0.33161$ (11) 0.16859 (11) 0.0452 (5) Uani $11 d . .$.

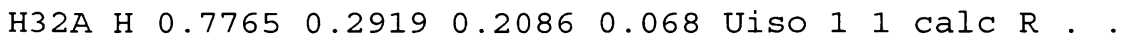

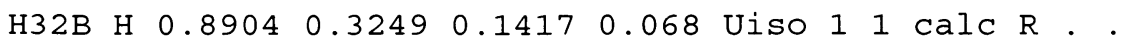

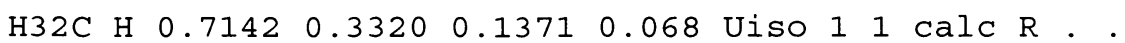
C33 C $0.6669(2) \quad 0.37834(11) \quad 0.40371(10) \quad 0.0411(4)$ Uani $11 d . .$. H33A H $0.74170 .3437 \quad 0.42460 .049$ Uiso 11 calc R . C34 C $0.5927(3) \quad 0.41313(15) \quad 0.46534(12) \quad 0.0609(6)$ Uani $11 d .$. H34A H 0.66750 .44040 .48460 .091 Uiso 1 l calc R. . H34B H $0.5485 \quad 0.3754 \quad 0.5030 \quad 0.091$ Uiso 11 calc R. . 
H34C H $0.51430 .44590 .4480 \quad 0.091$ Uiso 11 calc $\mathrm{R}$. .

C35 C $0.5546(2) \quad 0.33293(13) \quad 0.37352(13) \quad 0.0552(6)$ Uani $11 d .$.

H35A H 0.60570 .31030 .33490 .083 Uiso 11 calc R. .

$\begin{array}{llllllllllll}\mathrm{H} 35 \mathrm{~B} & \mathrm{H} & 0.4751 & 0.3642 & 0.3549 & 0.083 & \text { Uiso } 1 & 1 & \mathrm{CalC} & \mathrm{R} & \text {. }\end{array}$

H35C H 0.51130 .29530 .41150 .083 Uiso 11 calc $\mathrm{R}$. .

C36 C $0.7021(2) \quad 0.50198(10) \quad 0.31740(11) \quad 0.0375(4)$ Uani 11 d. .

H36A H 0.76360 .51520 .27050 .045 Uiso 11 calc R . .

C37 C $0.5398(2) \quad 0.50866(12) \quad 0.30041(12) \quad 0.0490(5)$ Uani $11 d .$.

H37A H $0.5179 \quad 0.47210 .27110 .073$ Uiso 11 calc $R$. .

H37B H $0.52380 .5570 \quad 0.2742 \quad 0.073$ Uiso 11 calc $R$. .

H37C H $0.47360 .50120 .3450 \quad 0.073$ Uiso 11 Calc R. .

C38 C $0.7399(3) \quad 0.55931(12) \quad 0.36277(14) \quad 0.0576(6)$ Uani 11 d . .

H38A H $0.84350 .55350 .3742 \quad 0.086$ Uiso 11 Calc $R$. .

H38B H 0.67280 .55330 .40710 .086 Uiso 1 I calc $R$. .

H38C H 0.72730 .60770 .33590 .086 Uiso 11 calc R . .

C39 C $0.88356(18) \quad 0.40418(9) \quad 0.31559(9) \quad 0.0267(3)$ Uani $11 d .$.

C40 C $1.2707(2) \quad 0.36569(10) \quad 0.09195(10) \quad 0.0377(4)$ Uani $11 d .$.

H4OA H 1.31380 .32300 .07180 .045 Uiso 11 calc $R$. .

C41 C $1.1822(3) \quad 0.40892(15) \quad 0.03666(12) \quad 0.0651(7)$ Uani $11 d$. .

H41A H $1.09680 .3800 \quad 0.02870 .098$ Uiso 11 Calc R. .

$\begin{array}{lllllllllll}\mathrm{H} 41 \mathrm{~B} & \mathrm{H} & 1.2462 & 0.4214 & -0.0083 & 0.098 & \text { Uiso } 1 & 1 \text { calc } \mathrm{R} .\end{array}$

$\mathrm{H} 41 \mathrm{C} \mathrm{H} 1.14630 .45330 .05380 .098$ Uiso 11 calc R. .

C42 C $1.3987(3) \quad 0.41099(13) \quad 0.10826(13) \quad 0.0580(6)$ Uani $11 d . .$.

H42A H $1.45590 .38210 .1436 \quad 0.087$ Uiso 11 calc R. .

H42B H $1.35870 .4536 \quad 0.12740 .087$ Uiso 11 calc $R$. .

H42C H 1.46410 .42650 .06430 .087 Uiso 11 Calc R. .

C43 C $1.0889(2) \quad 0.19677(10) \quad 0.30425(9) \quad 0.0373(4)$ Uani 11 d . .

H43A H 1.09720 .15750 .27390 .045 Uiso 11 Calc R. .

C44 C $1.1865(3) \quad 0.17699(12) \quad 0.36545$ (11) 0.0595 (6) Uani $11 d$.

H44A H 1.29130 .17590 .34610 .089 Uiso 11 calc $R$. .

H44B H 1.15650 .12930 .39190 .089 Uiso 11 Calc $R$. .

$\mathrm{H} 44 \mathrm{C} \mathrm{H} 1.17420 .21310 .39770 .089$ Uiso 11 Calc $\mathrm{R}$.

C45 C $0.9262(3) \quad 0.20193(11) \quad 0.33337(11) \quad 0.0501(5)$ Uani $11 d . .$.

H45A H 0.86380 .21040 .29360 .075 Uiso 11 calc R. .

H45B H 0.91490 .24210 .36090 .075 Uiso 11 calc $R$. .

H45C H 0.89520 .15660 .36440 .075 Uiso 11 calc $\mathrm{R}$. .

C46 C $1.3335(2) \quad 0.16163(10) \quad 0.18219(11) \quad 0.0414(4)$ Uani $11 d .$.

H46A H 1.34090 .17050 .23220 .050 Uiso 11 calc R. .

C47 C $1.2929(3) \quad 0.08192(11) \quad 0.18817(13) \quad 0.0543(6)$ Uani $11 d .$.

H47A H 1.19560 .07250 .21610 .081 Uiso 11 Calc R. .

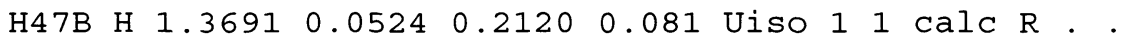

$\mathrm{H} 47 \mathrm{C} \mathrm{H} 1.28780 .06960 .14030 .081$ Uiso $11 \mathrm{calc} R$.

C48 C $1.4889(3) \quad 0.17783(13) \quad 0.14280(15) \quad 0.0630(7)$ Uani 11 d . .

H48A H 1.51140 .22960 .13880 .095 Uiso 11 calc R . .

$\mathrm{H} 48 \mathrm{~B}$ H 1.49170 .16450 .09490 .095 Uiso 11 calc $\mathrm{R}$.

H48C H $1.56340 .1498 \quad 0.16940 .095$ Uiso 11 Calc R. .

C49 C $1.1411(3) \quad 0.20576(11) \quad 0.09052(10) \quad 0.0445(5)$ Uani 11 d. . .

H49A H 1.10020 .25480 .07480 .053 Uiso 11 Calc R .

C50 C $1.2389(3) \quad 0.18701(15) \quad 0.02598(12) \quad 0.0713(8)$ Uani 11 d . .

H50A H 1.32150 .22200 .01340 .107 Uiso 11 calc $R$. .

H50B H $1.1790 \quad 0.1887-0.01450 .107$ Uiso 11 calc R. .

H50C H 1.27920 .13830 .03760 .107 Uiso 11 Calc R. .

C51 C $1.0053(3) \quad 0.15498(13) \quad 0.10969(14) \quad 0.0622(7)$ Uani 11 d. . .

H51A H 0.94620 .16750 .15200 .093 Uiso 11 calc R. .

H51B H 1.03920 .10490 .11980 .093 Uiso 11 Calc R. .

H51C H 0.94380 .16010 .06950 .093 Uiso 11 calc R . .

C52 C $1.18059(19) \quad 0.27047(9) \quad 0.18903(9) \quad 0.0283(3)$ Uani $11 d$. 


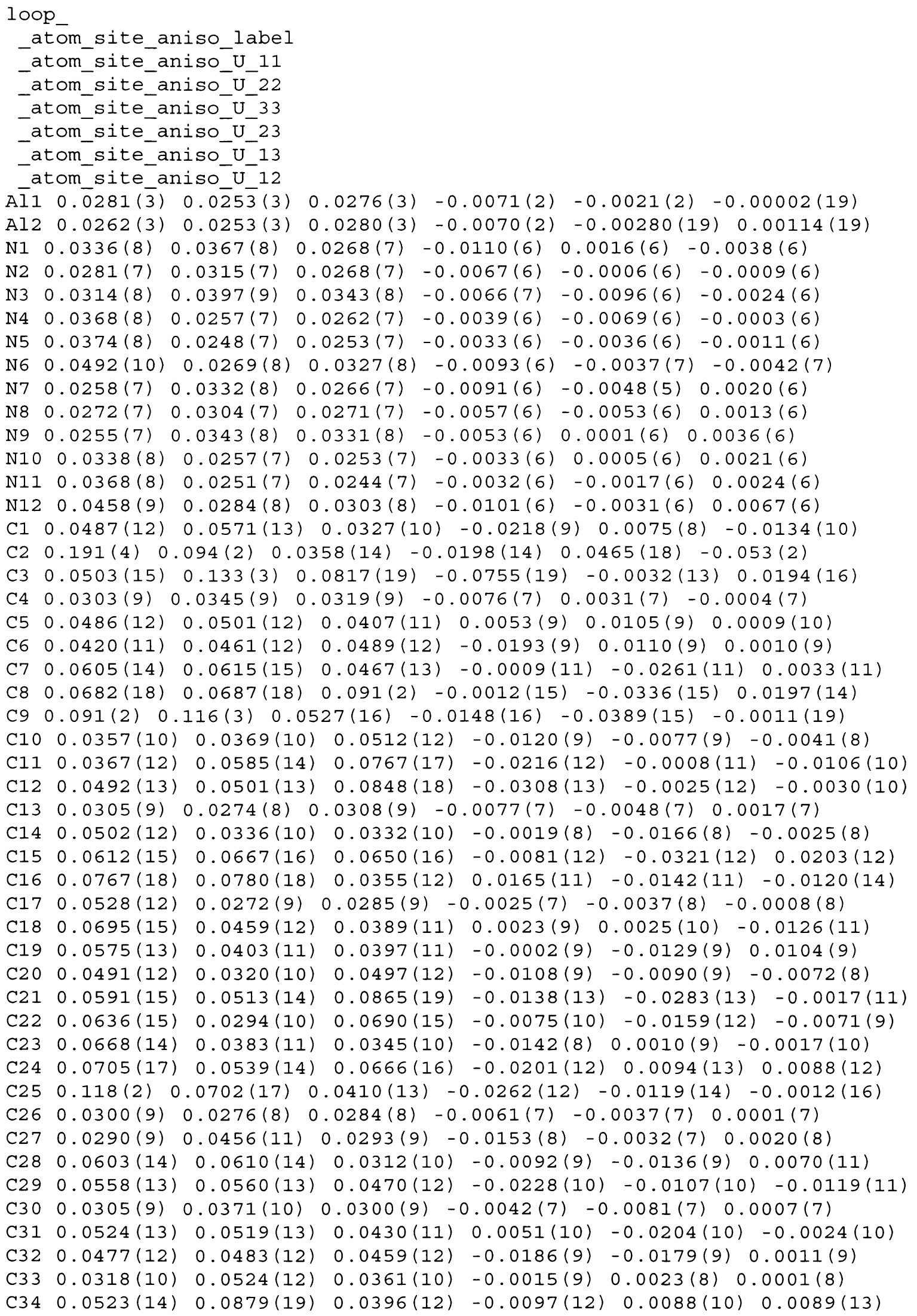




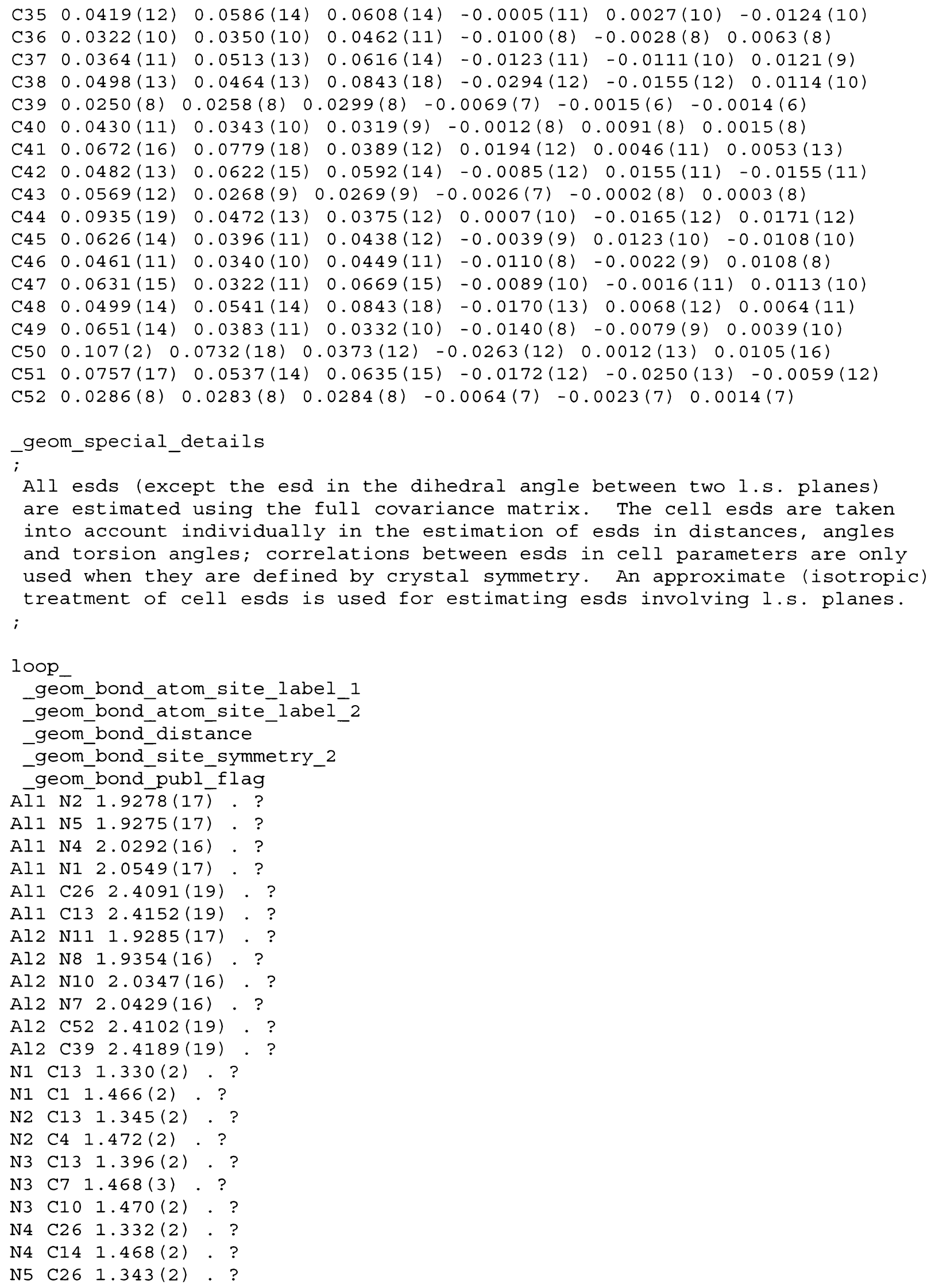




$$
\begin{aligned}
& \text { N5 C17 } 1.466(2) . ? \\
& \text { N6 C26 1.393(2) . ? } \\
& \text { N6 C20 } 1.476(2) \text {. ? } \\
& \text { N6 C23 } 1.476(2) \text {. ? } \\
& \text { N7 C39 } 1.330(2) \text {. ? } \\
& \text { N7 C27 } 1.469(2) \text {. ? } \\
& \text { N8 C39 } 1.349(2) \text {. ? } \\
& \text { N8 C30 } 1.470(2) \text {. ? } \\
& \text { N9 C39 } 1.393(2) \text {. ? } \\
& \text { N9 C36 } 1.471(2) \text {. ? } \\
& \text { N9 C33 } 1.476(2) \text {. ? } \\
& \mathrm{N} 10 \mathrm{C} 52 \quad 1.331(2) \text {. ? } \\
& \mathrm{N} 10 \mathrm{C} 40 \mathrm{1.472}(2) \text {. ? } \\
& \mathrm{N} 11 \mathrm{C} 521.345(2) \text {. ? } \\
& \mathrm{N} 11 \mathrm{C} 431.465(2) \text {. ? } \\
& \mathrm{N} 12 \mathrm{C} 52 \text { 1.394(2) . ? } \\
& \mathrm{N} 12 \mathrm{C} 491.475(2) \text {. ? } \\
& \mathrm{N} 12 \mathrm{C} 46 \quad 1.476(2) \text {. ? } \\
& \text { C1 C2 } 1.510(3) \text {. ? } \\
& \text { C1 C3 } 1.524(3) \text {. ? } \\
& \text { C4 C5 } 1.523(3) \text {. ? } \\
& \text { C4 C6 } 1.521(3) \text { ? } \\
& \text { C7 C8 } 1.522(4) \text {. ? } \\
& \text { C7 C9 } 1.524(3) \text {. ? } \\
& \text { C10 C11 } 1.527(3) \text {. ? } \\
& \mathrm{C} 10 \mathrm{C} 121.529(3) . ? \\
& \text { C14 C15 1.517(3) . ? } \\
& \text { C14 C16 1.522(3) . ? } \\
& \text { C17 C19 1.521(3) . ? } \\
& \text { C17 C18 1.529(3) . ? } \\
& \text { C20 C22 1.526(3). ? } \\
& \text { C20 C21 1.530(3).? } \\
& \text { C23 C25 1.527(3). ? } \\
& \text { C23 C24 1.530(3). ? } \\
& \mathrm{C} 27 \mathrm{C} 281.516(3) \text { ? } \\
& \text { C27 C29 1.521(3) . ? } \\
& \text { C30 C32 } 1.526(3) \text {. ? } \\
& \text { C30 C31 } 1.527(3) \text {. ? } \\
& \text { C33 C34 1.525(3) . ? } \\
& \text { C33 C35 } 1.533(3) \text {. ? } \\
& \begin{array}{lll}
\mathrm{C} 36 & \mathrm{C} 37 & 1.527(3) . ?
\end{array} \\
& \text { C36 C38 1.537(3) . ? } \\
& \mathrm{C} 40 \mathrm{C} 411.513(3) \text { ? } \\
& \text { C40 C42 1.518(3). ? } \\
& \text { C43 C45 1.522(3). ? } \\
& \text { C43 C44 1.526(3) . ? } \\
& \text { C46 C47 1.523(3) . ? } \\
& \text { C46 C48 1.529(3) . ? } \\
& \mathrm{C} 49 \mathrm{C} 501.525(3) \text { ? } \\
& \text { C49 C51 } 1.532(3) \text {. ? }
\end{aligned}
$$




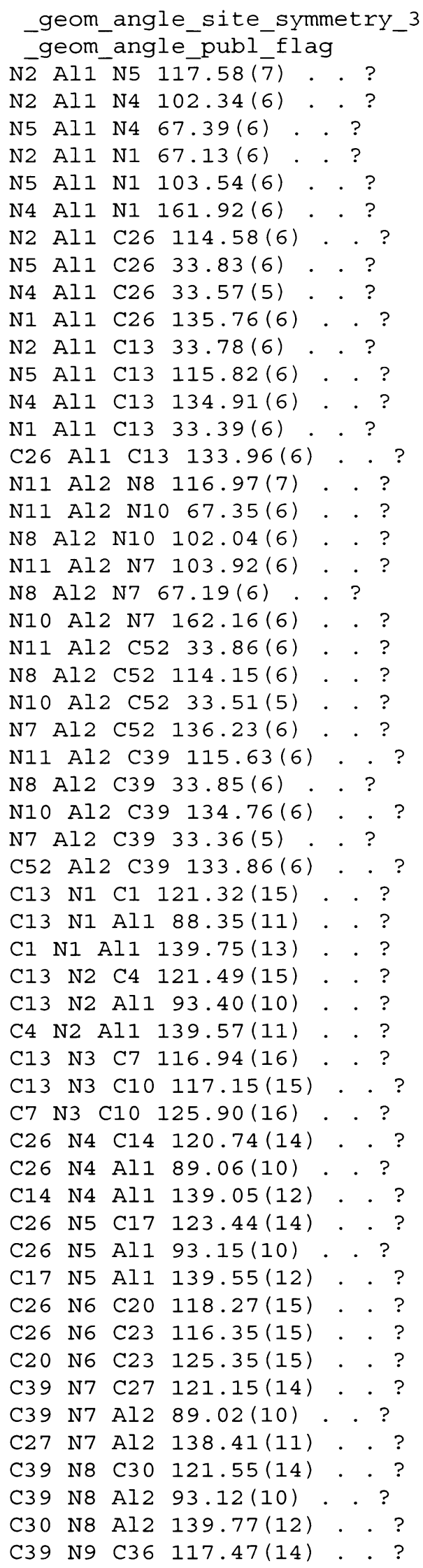




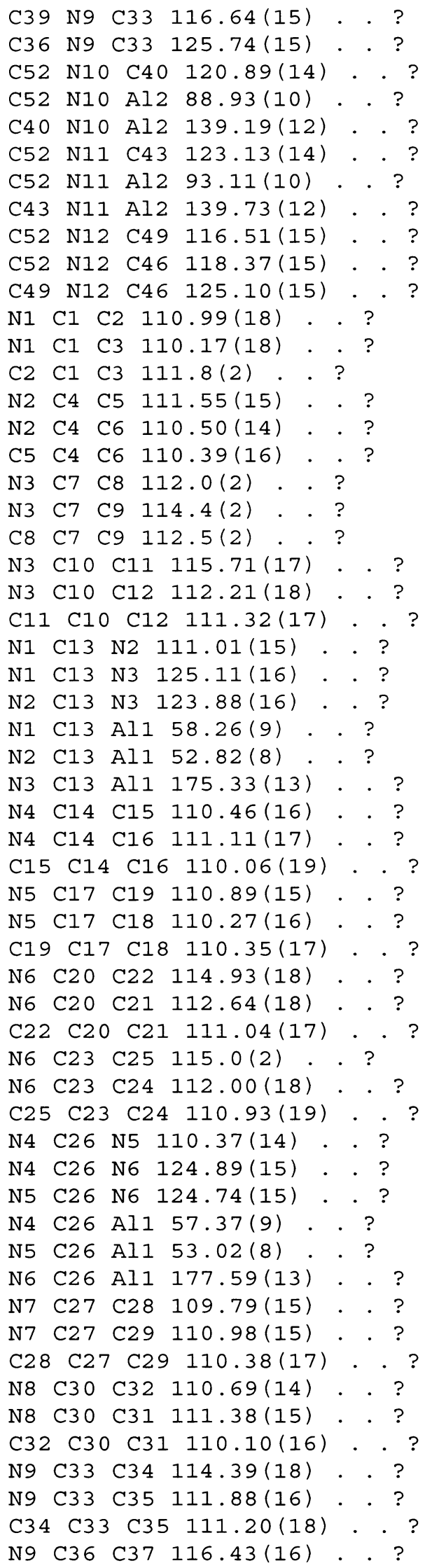




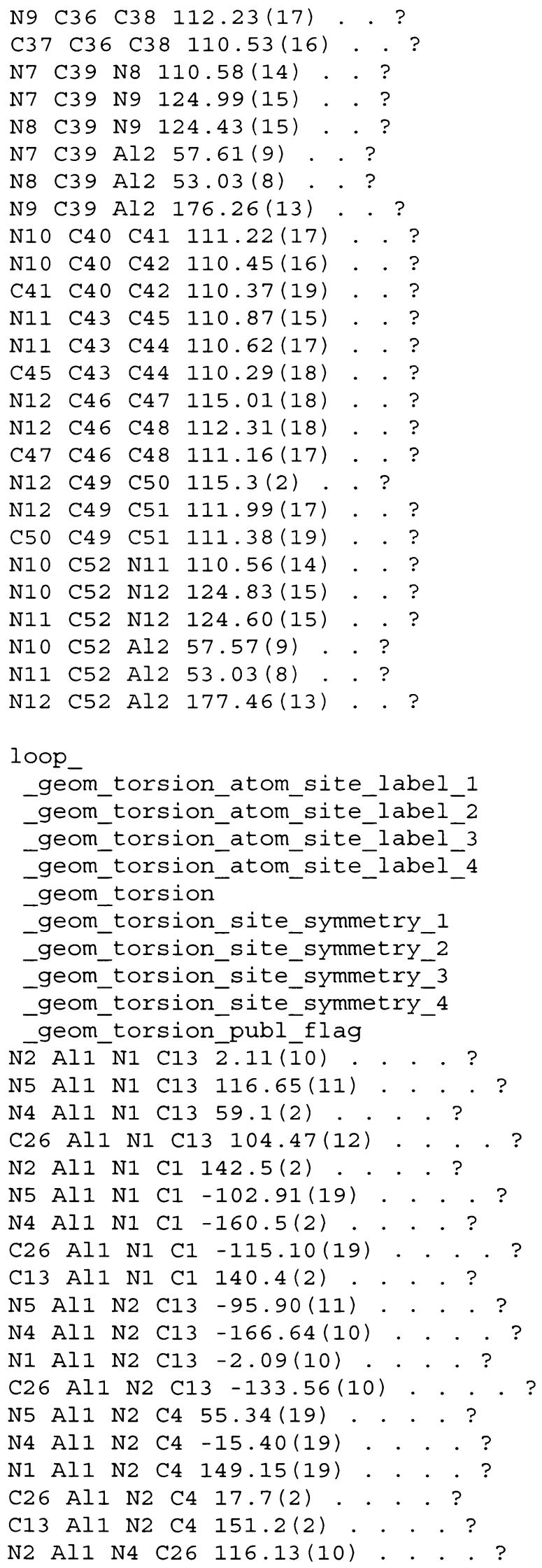




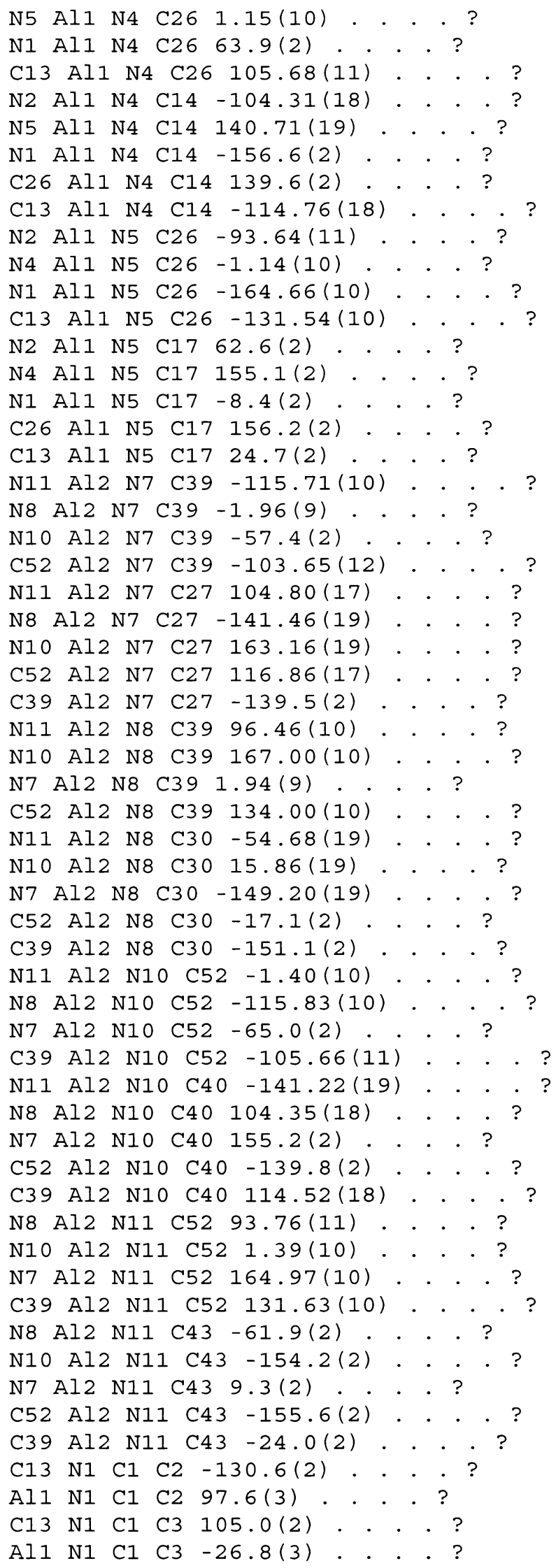




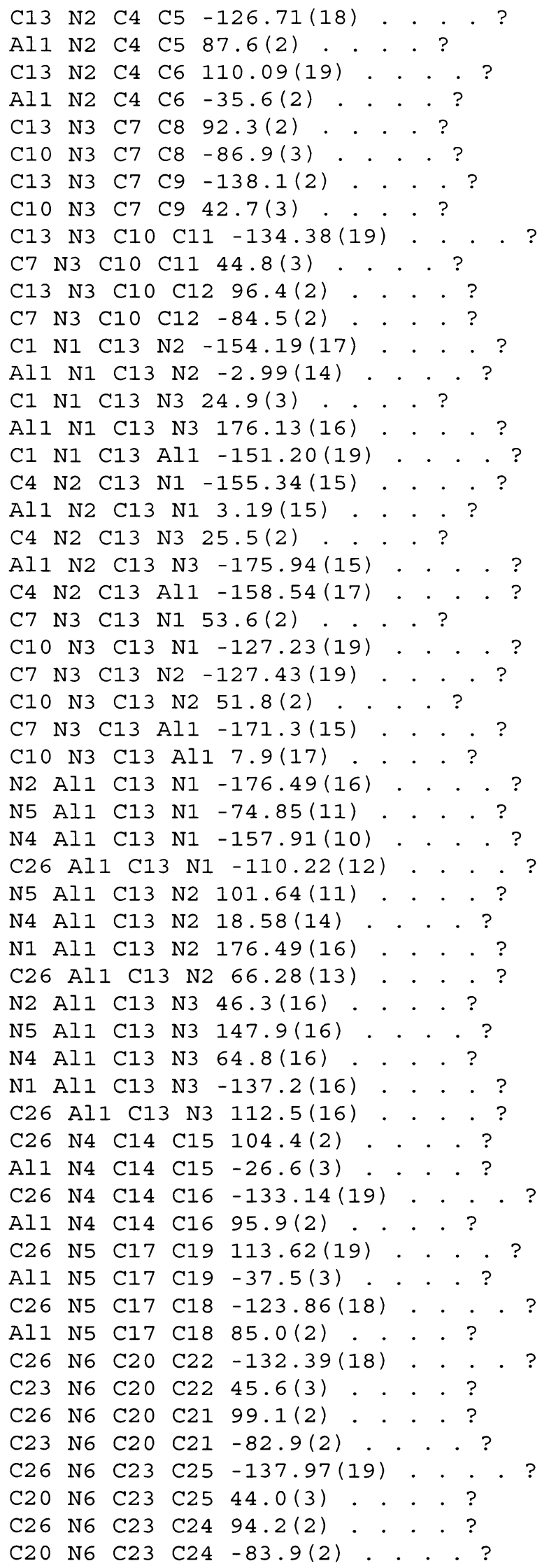




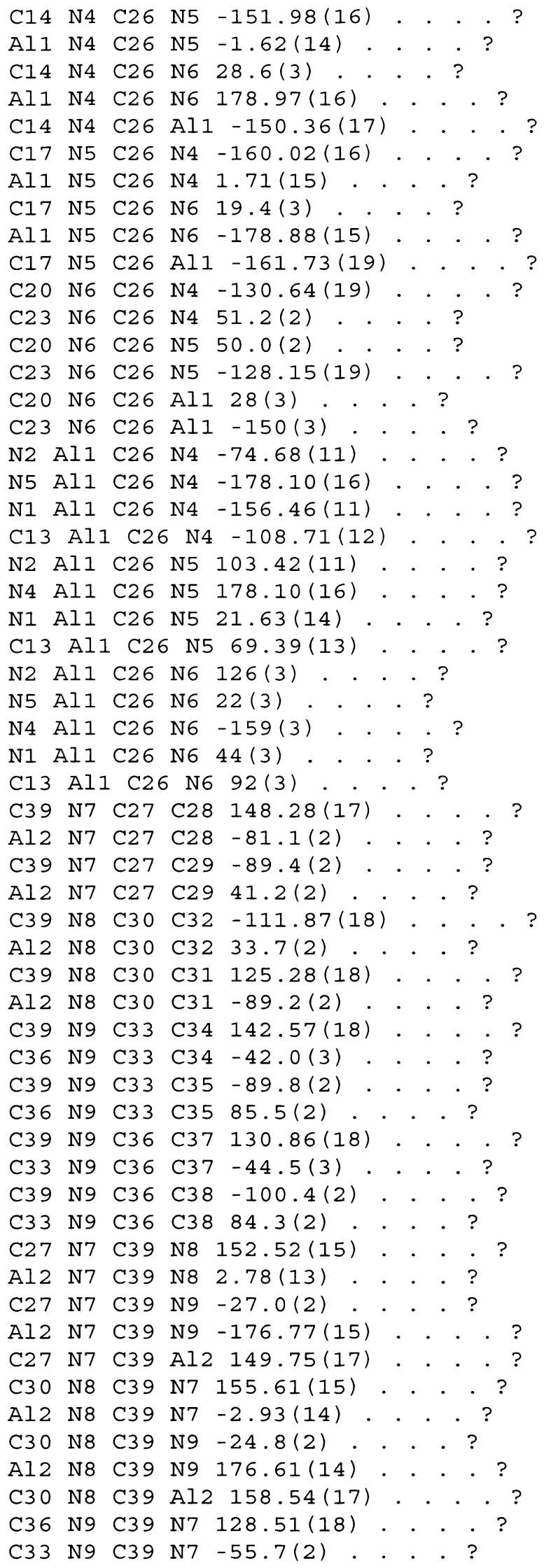




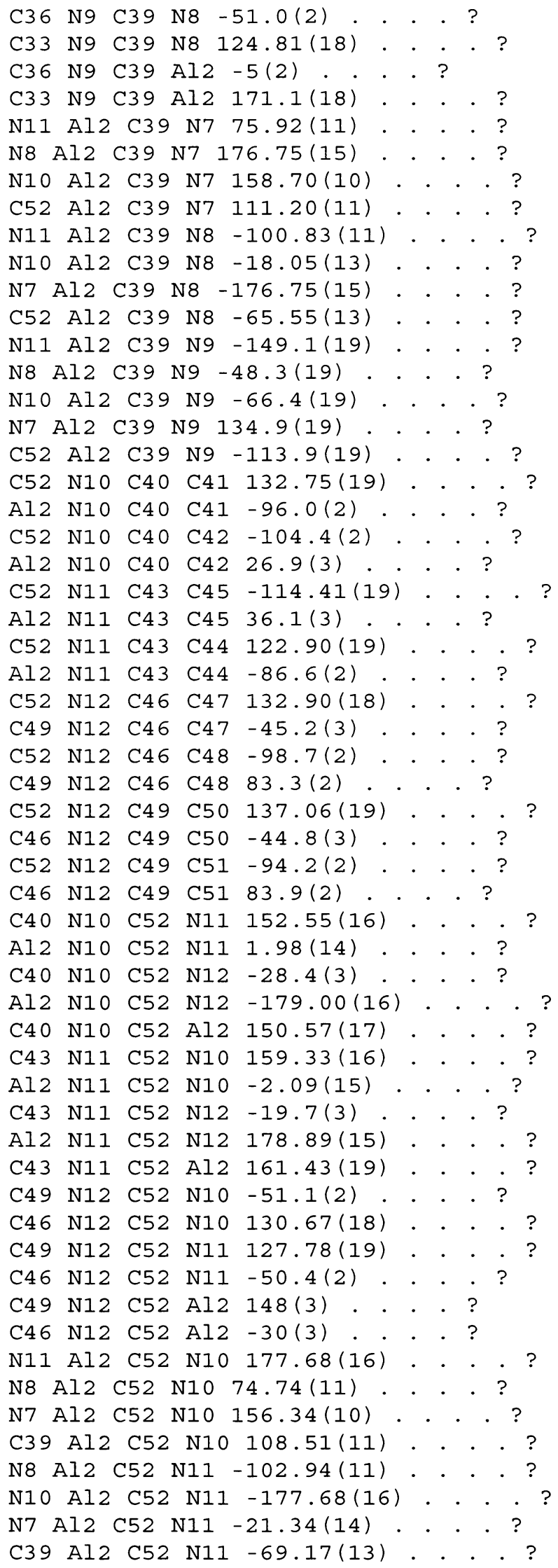


$\mathrm{N} 11$ Al2 $\mathrm{C} 52 \mathrm{~N} 12-21(3)$. . . ?

N8 Al2 C52 N12 $-124(3)$. . . ?

N10 Al2 C52 N12 $161(3)$. . . ?

N7 Al2 C52 N12 $-43(3)$. . . ?

C39 Al2 C52 N12 $-90(3)$. . . ?

_diffrn_measured_fraction_theta_max 0.995

_diffrn_reflns_theta_full 25.00

_diffrn_measured_fraction_theta_full 1.000

_refine_diff_density_max $0.3 \overline{81}$

refine_diff density min $\quad-0.168$

_refine_diff_density_rms 0.047 


\section{Compound 5: $\left[\mathrm{MeC}\left(\mathrm{N}^{\mathrm{i}} \mathrm{Pr}\right)_{2}\right]_{2} \mathrm{AlH}$}

View perpendicular to Al-H1 bond

View along Al-H1 bond
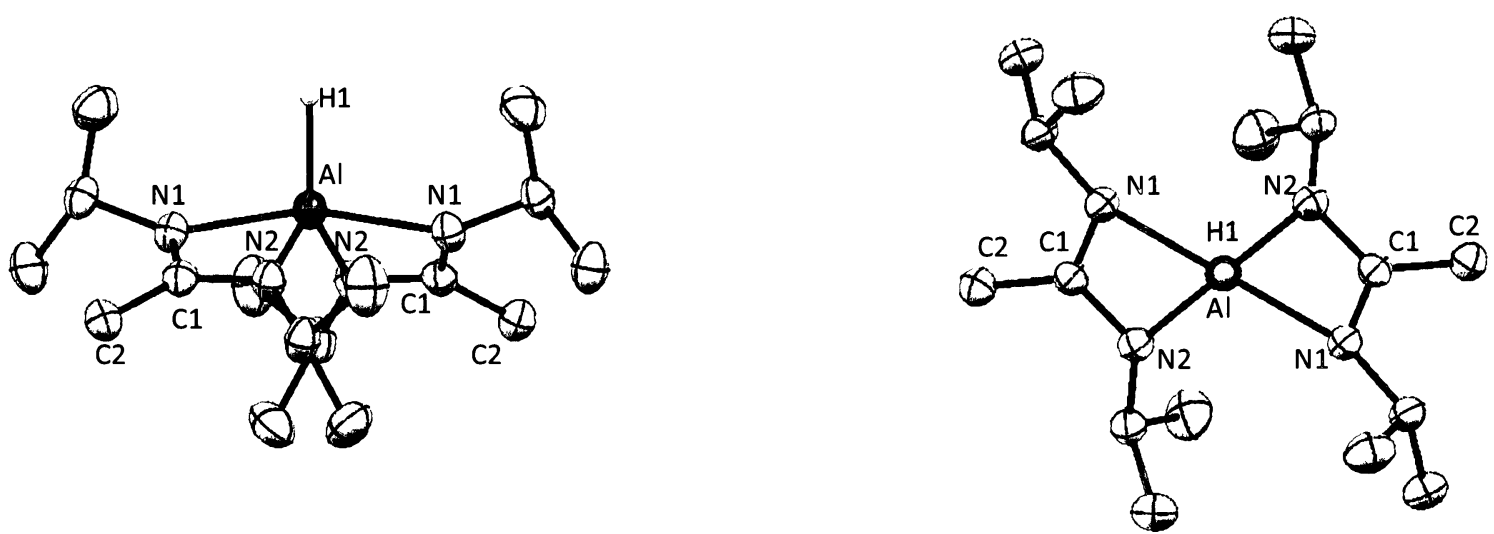

View of unit cell along a axis

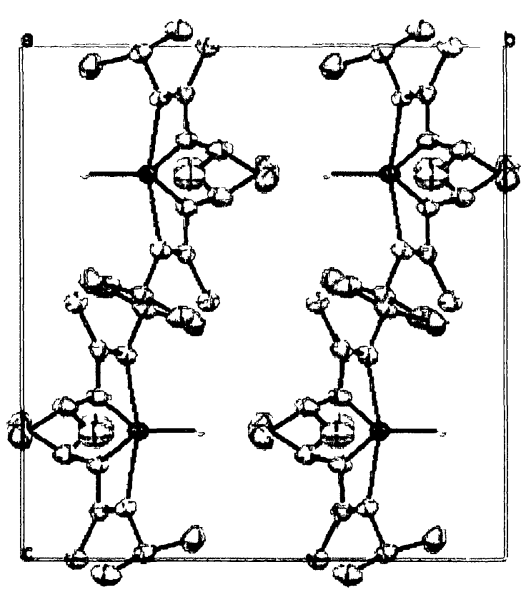

View of unit cell along $b$ axis

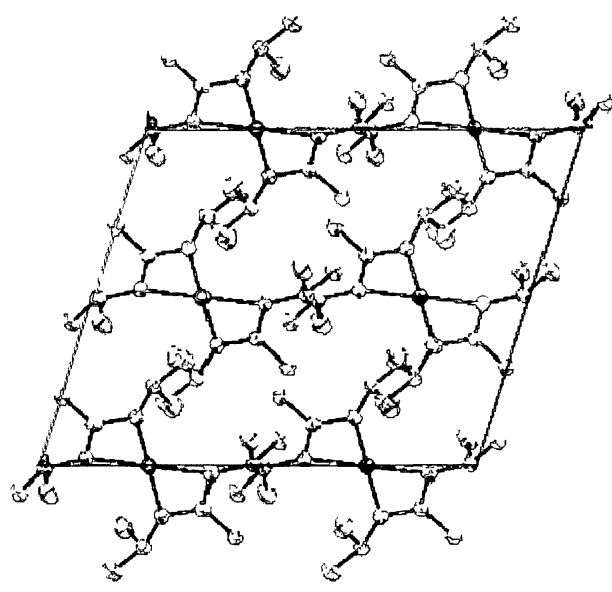

View of unit cell along $c$ axis

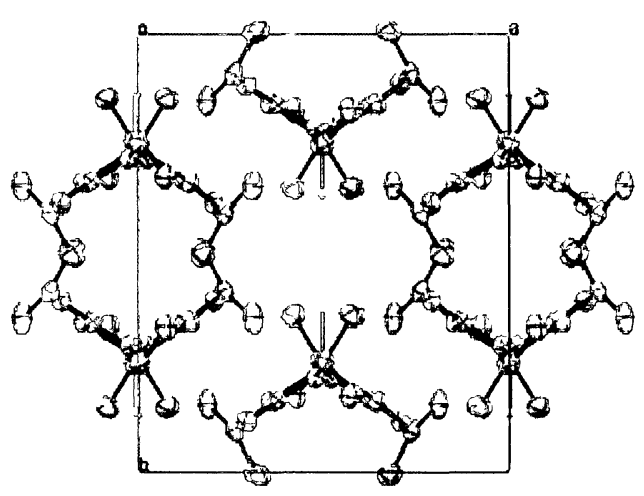




\section{Compound 5: $\left[\mathrm{MeC}\left(\mathrm{N}^{\mathrm{i}} \mathrm{Pr}\right)_{2}\right]_{2} \mathrm{AlH}$.cif file}

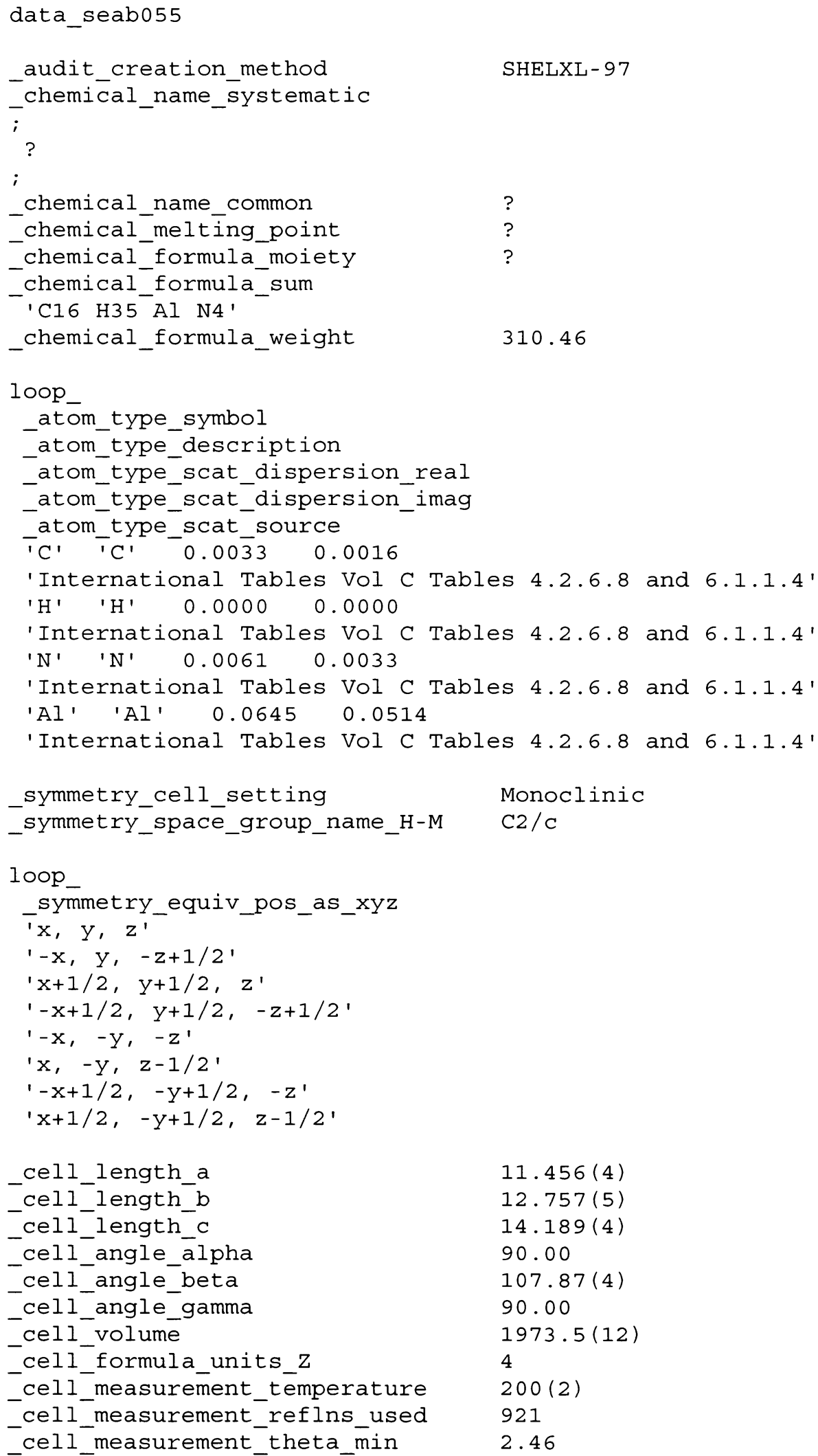




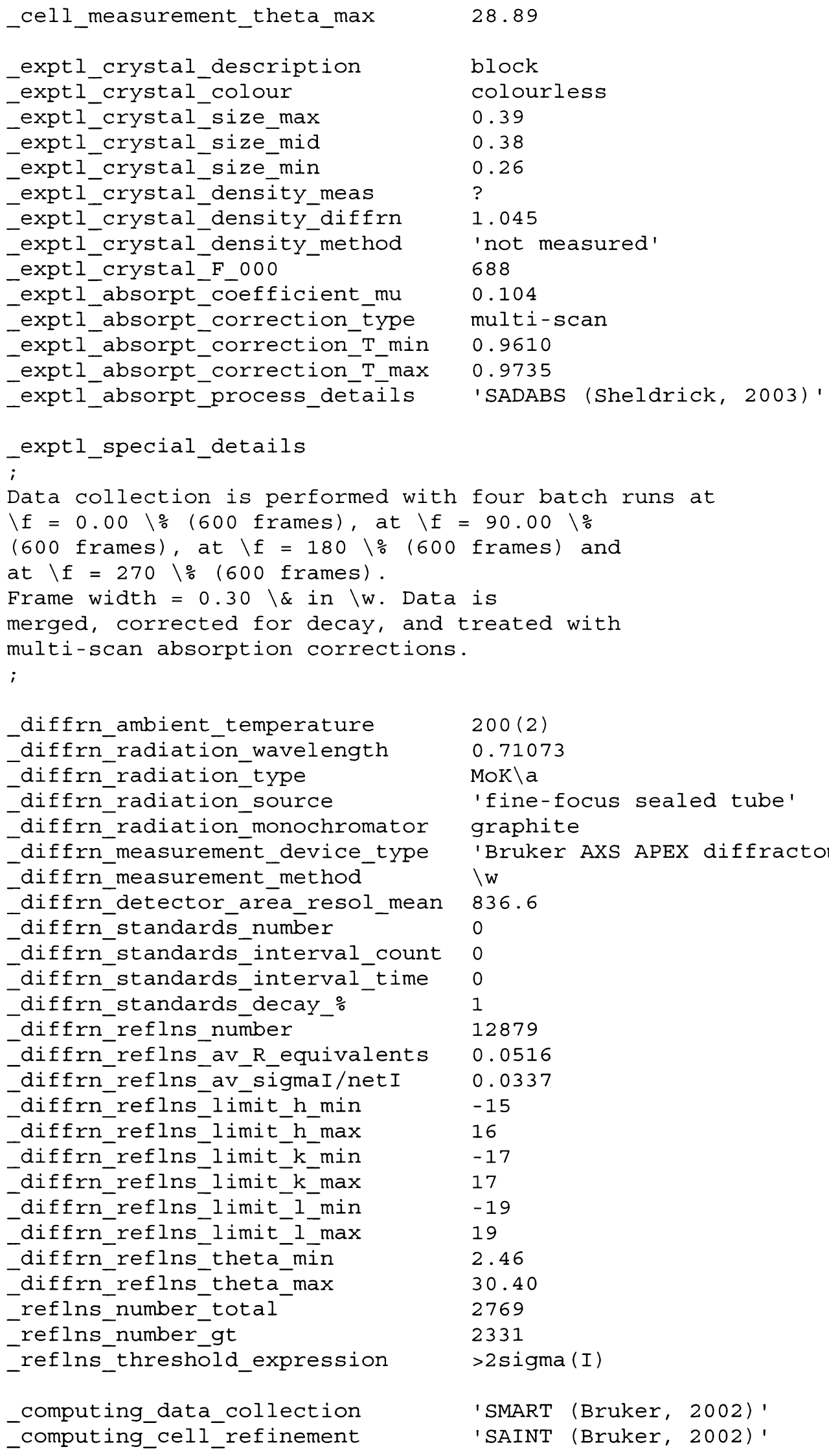


_computing_data_reduction _computing_structure_solution _computing_structure_refinement _computing_molecular_graphics _computing_publication_material
SAINT

'SHELXS-97 (Sheldrick, 1990)'

' SHELXL-97 (Sheldrick, 1997)'

'SHELXTL (Sheldrick, 2001)'

'SHELXTL (Sheldrick, 2001)'

_refine_special_details

i

Refinement of $\mathrm{F}^{\wedge} 2^{\wedge}$ against $\mathrm{ALL}$ reflections. The weighted $\mathrm{R}$-factor wR and goodness of fit $S$ are based on $F^{\wedge} 2^{\wedge}$, conventional $R$-factors $R$ are based on $F$, with $F$ set to zero for negative $F^{\wedge} 2^{\wedge}$. The threshold expression of $\mathrm{F}^{\wedge} 2^{\wedge}>2 \mathrm{sigma}\left(\mathrm{F}^{\wedge} 2^{\wedge}\right)$ is used only for calculating R-factors (gt) etc. and is not relevant to the choice of reflections for refinement. R-factors based on $F^{\wedge} 2^{\wedge}$ are statistically about twice as large as those based on $F$, and $R-$ factors based on ALL data will be even larger. ;

refine_ls structure factor_coef Fsqd

refine ls matrix type full

_refine_ls_weighting_scheme calc

refine ls weighting details

$' \operatorname{calc} \bar{w}=1 /\left[\backslash \mathrm{S}^{\wedge} 2^{\wedge}\left(\mathrm{FO} \mathrm{O}^{\wedge} 2^{\wedge}\right)+(0.0922 \mathrm{P})^{\wedge} 2^{\wedge}+0.7490 \mathrm{P}\right]$ where $\mathrm{P}=\left(\mathrm{FO} \mathrm{O}^{\wedge} 2^{\wedge}+2 \mathrm{FC}^{\wedge} 2^{\wedge}\right) / 3^{\prime}$

_atom_sites_solution_primary

direct

atom sites solution secondary

-atom_sites_solution_hydrogens

difmap

refine ls hydrogen treatment

_refine_ls_extinction_method

refine_ls_extinction_coef

refine Is number refins

refine Is number parameters

-refine_ls_number_restraints

-refine_ls_R_factor_all

geom

mixed

none

?

2769

102

0

_refine_ls_R_factor_gt

0.0537

_refine_ls_wR_factor_ref

0.0440

refine_ls_wR_factor_gt

0.1546

0.1286

refine_ls_restrained_ $\bar{S}$ al $\bar{l}$

1. 054

_refine_ls_shift/su_max

1. 054

refine_ls_shift/su mean

0.000

0.000

loop

atom site label

-atom_site type symbol

_atom_site_fract_x

_atom_site_fract_y

_atom_site_fract_z

_atom_site_u_iso_or_equiv

-atom_site_adp_type

-atom_site_occupancy

-atom site symmetry multiplicity

_atom_site_calc_flag

-atom site refiñement flags

_atom_site_disorder_assembly

atom_site_disorder_group

$A \bar{I}$ Al $\overline{0} .500 \overline{0} 0.23863 \overline{(4)} 0.7500 \quad 0.02874(17)$ Uani 12 d $\mathrm{S}$.

H1 H $0.5000 \quad 0.3687(18) \quad 0.7500 \quad 0.043$ Uiso 12 d $S$. .

$\mathrm{N} 1 \mathrm{~N} \quad 0.35526(9) \quad 0.15750(8) \quad 0.68358(7) \quad 0.0317(2) \quad \operatorname{Uani} 11 \mathrm{~d}$. 
$\mathrm{N} 2 \mathrm{~N} \quad 0.47889(9) \quad 0.21289(8) \quad 0.60234(7) \quad 0.0302(2) \quad$ Uani $11 d$. C1 C $0.26195(12) \quad 0.08986(11) \quad 0.70327(9) \quad 0.0368(3)$ Uani $11 d .$. H1A H 0.20440 .06630 .63840 .044 Uiso 11 calc R . . C2 C $0.18860(14) \quad 0.15130(15) \quad 0.75819(12) \quad 0.0535(4)$ Uani $11 d . .$. H2A H $0.1498 \quad 0.21190 .71830 .080$ Uiso 11 Calc R. . H2B H $0.2437 \quad 0.17550 .82190 .080$ Uiso 11 calc $R$. $\mathrm{H} 2 \mathrm{C} \mathrm{H} 0.12520 .10600 .76960 .080$ Uiso 1 calc $\mathrm{R}$. . C3 C $0.32222(17) \quad-0.00724(13) \quad 0.76133(13) 0.0536(4)$ Uani 11 d . . H3A H $0.3633-0.04750 .72190 .080$ Uiso 11 calc $R$. . H3B H $0.2594-0.05090 .77570 .080$ Uiso 11 calc $R$. . $\mathrm{H} 3 \mathrm{C} \mathrm{H} 0.38260 .01450 .82360 .080$ Uiso 11 Calc $\mathrm{R}$. C4 C $0.50677(12) \quad 0.25260(10) \quad 0.51394(10) \quad 0.0344(3)$ Uani 11 d. . . H4A H $0.42730 .2690 \quad 0.46230 .041$ Uiso 11 calc R . . C5 C $0.57961(15) \quad 0.35456(12) \quad 0.54063(12) \quad 0.0483(4)$ Uani 11 d . .

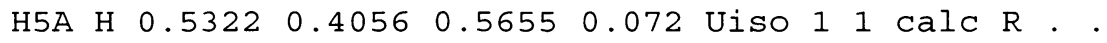
H5B H $0.5957 \quad 0.38290 .48160 .072$ Uiso 11 calc $R$. . $\mathrm{H} 5 \mathrm{C} \mathrm{H} 0.65760 .3406 \quad 0.59190 .072$ Uiso 1 l calc $\mathrm{R}$. . C6 C $0.57558(14) \quad 0.17247(13) \quad 0.47035(11) \quad 0.0450(3)$ Uani 11 d . . H6A H 0.52760 .10770 .45450 .067 Uiso 11 calc $R$. .

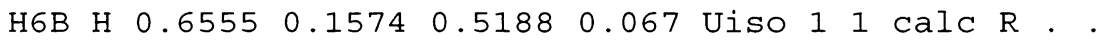
H6C H 0.58770 .20110 .40990 .067 Uiso 1 l calc $R$. . C7 C $0.37602(11) \quad 0.16079(9) \quad 0.59503(9) \quad 0.0293(3)$ Uani 11 d . . C8 C $0.29316(12) \quad 0.11258(10) \quad 0.50137(9) \quad 0.0351(3)$ Uani 11 d. . . $\begin{array}{lllllllllllll}\mathrm{H} 8 \mathrm{~A} & \mathrm{H} & 0.3123 & 0.1421 & 0.4441 & 0.053 & \text { Uiso } 1 & 1 & \mathrm{CalC} & \mathrm{R} & \text {. }\end{array}$ H8B H $0.2075 \quad 0.12770 .4960 \quad 0.053$ Uiso 11 Calc R . . H8C H $0.3058 \quad 0.03650 .50310 .053$ Uiso 11 calc $R$. .

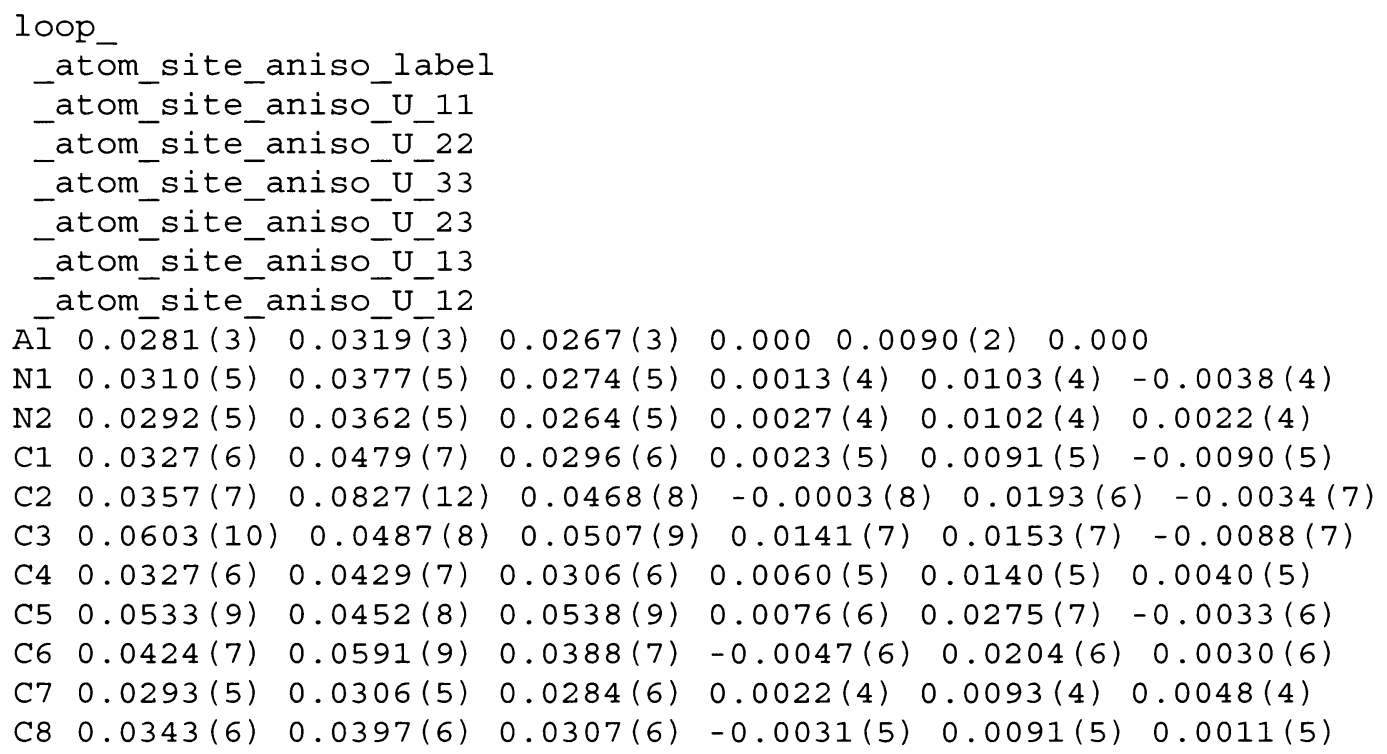

_geom_special_details

All esds (except the esd in the dihedral angle between two 1.s. planes) are estimated using the full covariance matrix. The cell esds are taken into account individually in the estimation of esds in distances, angles and torsion angles; correlations between esds in cell parameters are only used when they are defined by crystal symmetry. An approximate (isotropic) treatment of cell esds is used for estimating esds involving l.s. planes. ; 


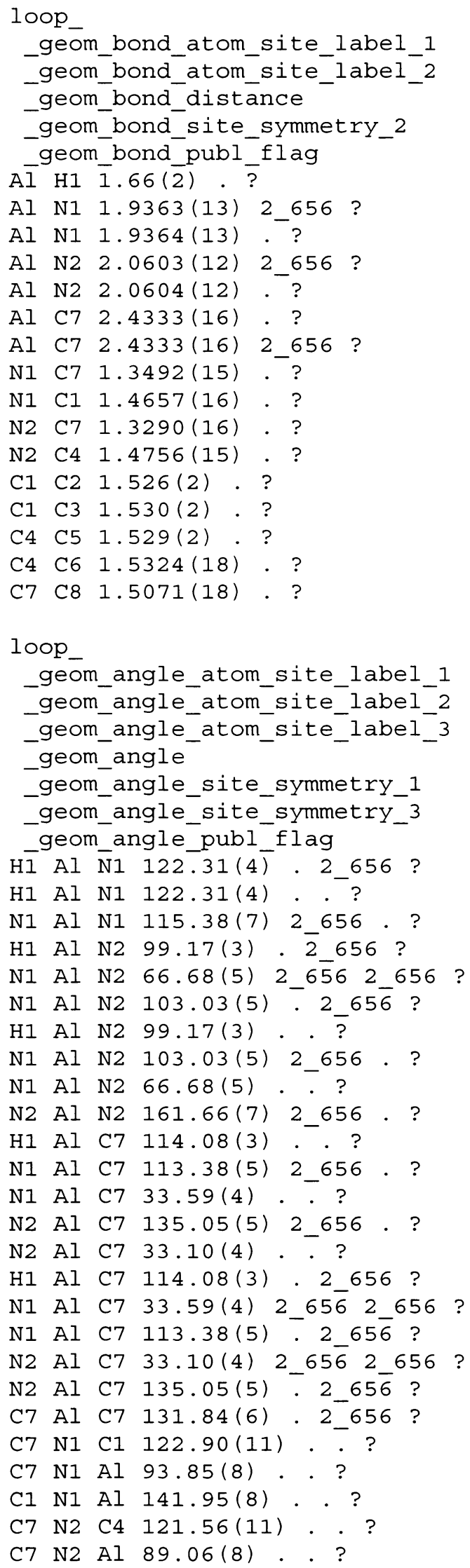




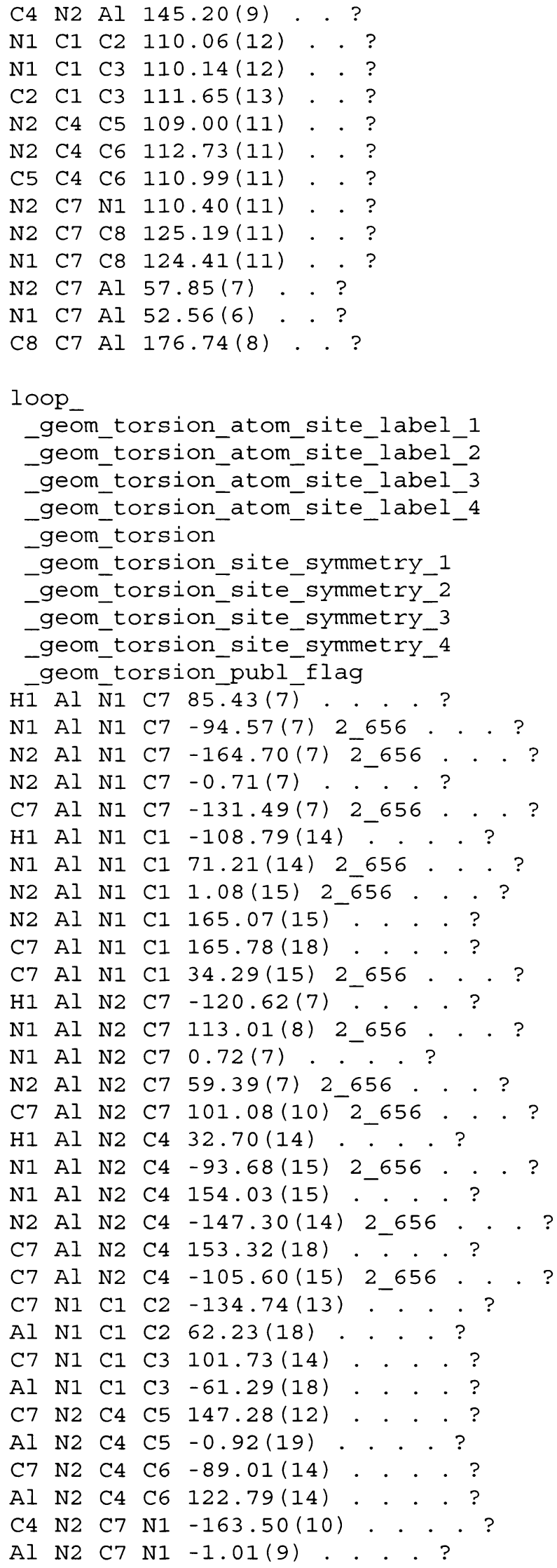




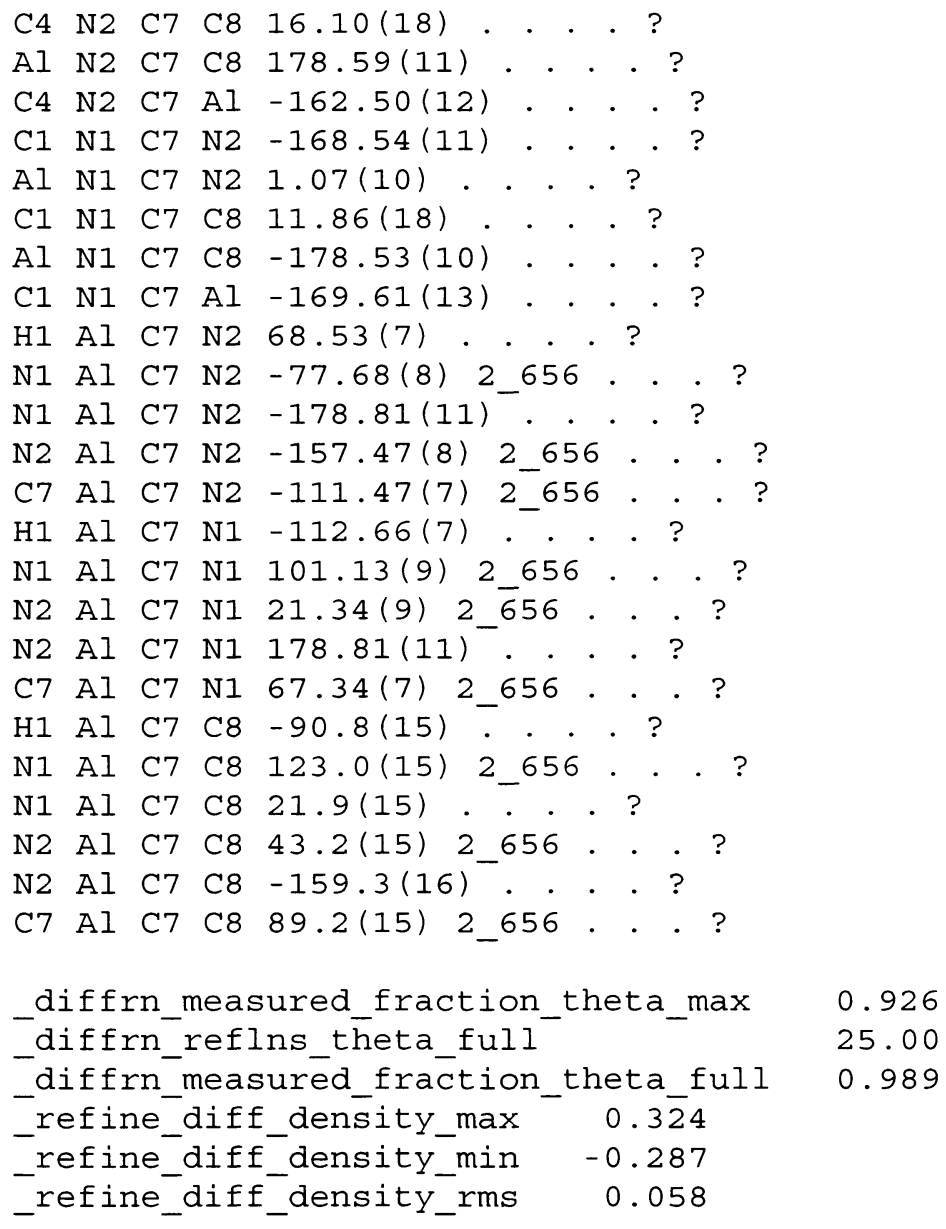




\section{Appendix B: ${ }^{1}$ H NMR Spectra for Thermolysis Studies}

Summary of boil up data.

B-

1

Chemical shift of hydride for compound 1-5.

B-

2

Compound 1: $\left[\mathrm{Me}_{2} \mathrm{NC}\left(\mathrm{N}^{\mathrm{i}} \mathrm{Pr}\right)_{2}\right]_{2} \mathrm{AlH}$

- at room temperature.

- at $56^{\circ} \mathrm{C}$ after 310 hours.

\section{Compound 2: $\left[\mathrm{Et}_{2} \mathrm{NC}\left(\mathrm{N}^{\mathrm{i}} \mathrm{Pr}\right)_{2}\right]_{2} \mathrm{AlH}$}

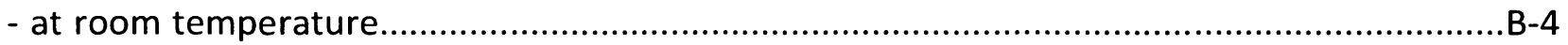

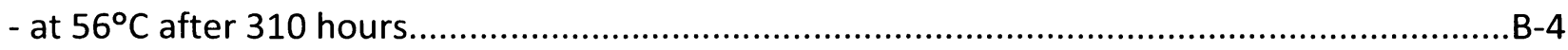

\section{Compound 3: ['PrHNC(N'Pr) $]_{2}$ AlH}

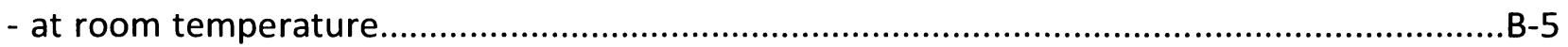

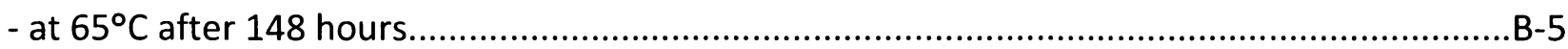

\section{Compound 4: [i'Pr$\left.{ }_{2} \mathrm{NC}\left(\mathrm{N}^{\mathrm{i}} \mathrm{Pr}\right)_{2}\right]_{2} \mathrm{AlH}$}

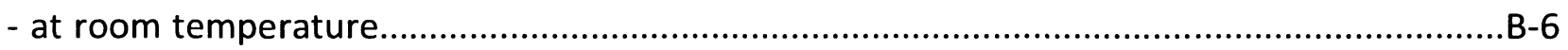

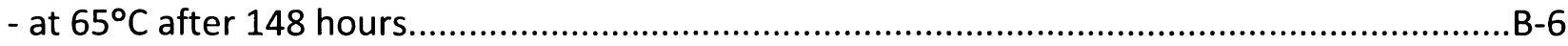

\section{Compound 5: $\left[\mathrm{MeC}\left(\mathrm{N}^{\mathrm{i}} \mathrm{Pr}\right)_{2}\right]_{2} \mathrm{AlH}$}

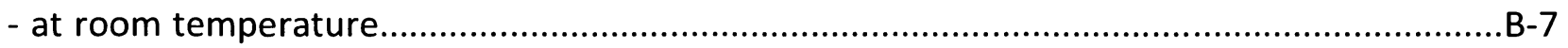

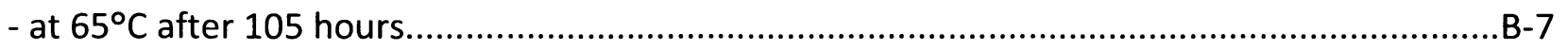




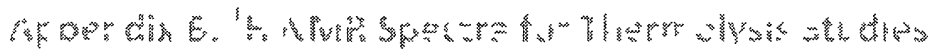

Summary of boil up data:

Table 1. Kinetic data collected for compounds 1-5

\begin{tabular}{|c|c|c|c|c|c|c|}
\hline Symbol & Compound & $\mathbf{R}$ & $\begin{array}{l}\text { Isothermal } \\
\text { Temperature }\end{array}$ & $\begin{array}{c}\text { Rate } \\
\text { Constant, k }\end{array}$ & $\begin{array}{c}\text { Half-Life, } \\
t_{1 / 2}\end{array}$ & $\begin{array}{c}\text { Correlation } \\
\text { Coefficient, } \\
\mathbf{R}^{2}\end{array}$ \\
\hline घ & 1 & $\mathrm{NMe}_{2}$ & $56^{\circ} \mathrm{C}$ & $4.4 \pm 0.2 \times 10^{-6} \mathrm{~s}^{-1}$ & 1.8 days & 0.98 \\
\hline$\bullet$ & 2 & $\mathrm{NEt}_{2}$ & $56^{\circ} \mathrm{C}$ & $1.07 \pm 0.04 \times 10^{-6} \mathrm{~s}^{-1}$ & 7.5 days & 0.99 \\
\hline$\bullet$ & 3 & $\mathrm{~N}^{\prime} \mathrm{Pr}, \mathrm{H}$ & $65^{\circ} \mathrm{C}$ & $1.49 \pm 0.02 \times 10^{-6} \mathrm{~s}^{-1}$ & 5.4 days & 1.00 \\
\hline$\Delta$ & 4 & $\mathrm{~N}^{\prime} \mathrm{Pr}_{2}$ & $65^{\circ} \mathrm{C}$ & $1.66 \pm 0.07 \times 10^{-6} \mathrm{~s}^{-1}$ & 4.8 days & 0.99 \\
\hline$x$ & 5 & $\mathrm{Me}$ & $65^{\circ} \mathrm{C}$ & $N / A$ & $N / A$ & $N / A$ \\
\hline
\end{tabular}

A)

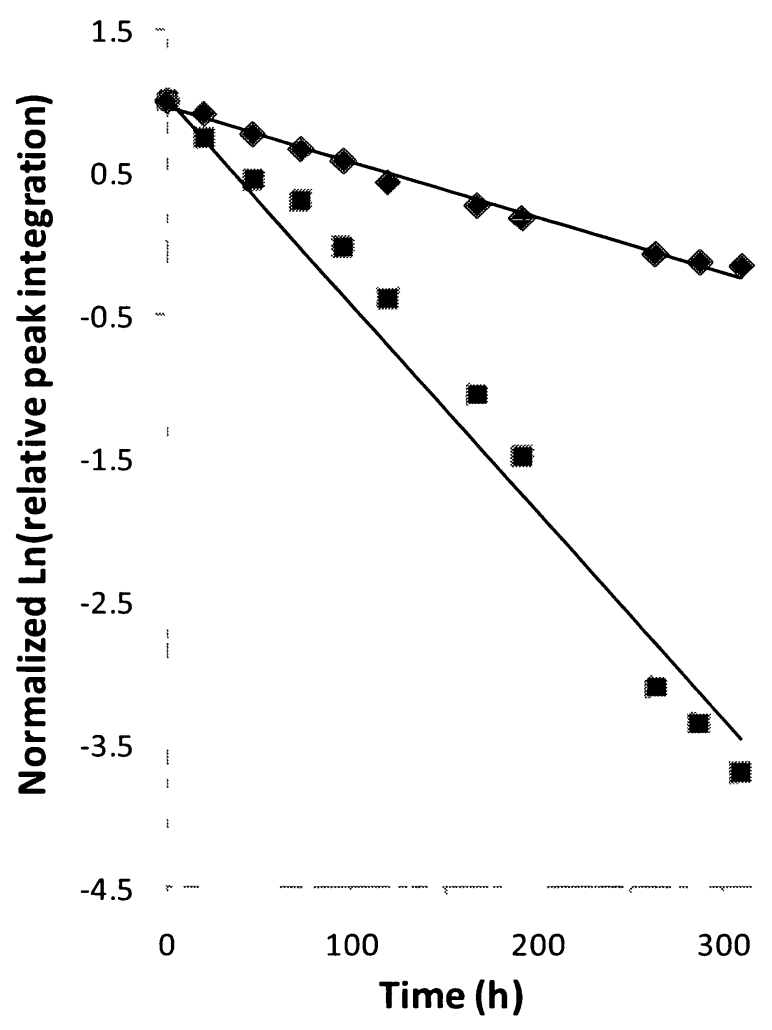

B)

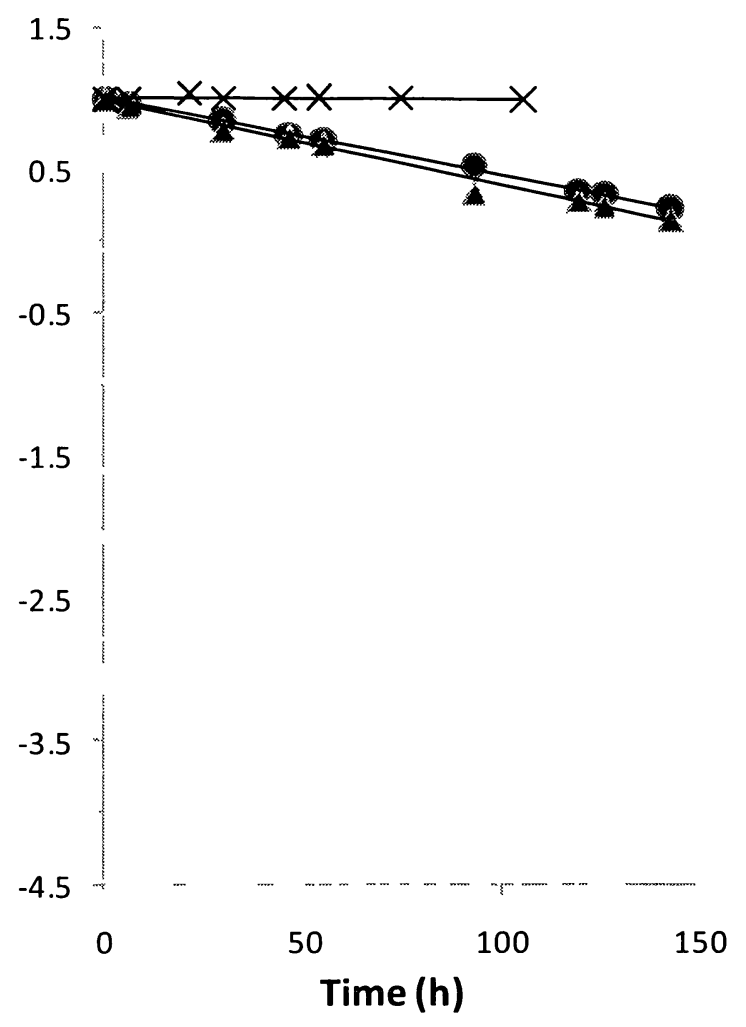

Figure 1. ${ }^{1} \mathrm{H}$ NMR Boilup data collected for the decomposition of A) compounds 1 and 2 at $56^{\circ} \mathrm{C}$, and B) compounds 3,4 , and 5 at $65^{\circ} \mathrm{C}$ 
Chemical shift of hydride for compound 1-5:

Table 2. Chemical shift of Al-H for compound 1-5

\begin{tabular}{cc}
\hline Compound & $\begin{array}{c}\boldsymbol{\delta} \text { of Al-H } \\
\text { (ppm) }\end{array}$ \\
\hline $\mathbf{1}$ & 4.25 \\
2 & 4.62 \\
3 & 4.74 \\
4 & 4.48 \\
5 & 4.88 \\
\hline
\end{tabular}
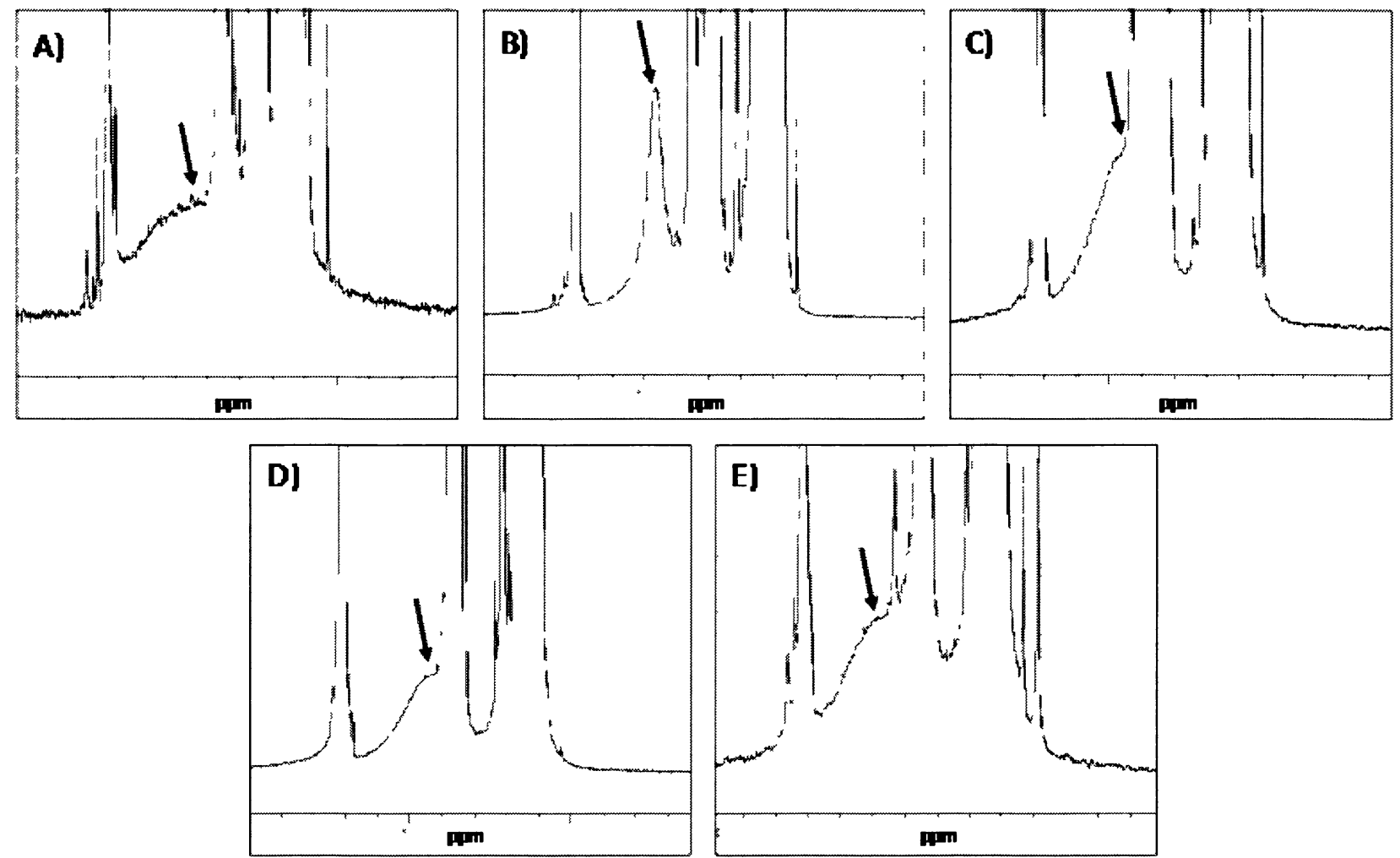

Figure 2. Hydride peak in baseline of ${ }^{1} H$ NMR for A) compound 1 , B) compound 2, C) compound 3, D) compound 4, and E) compound 5 


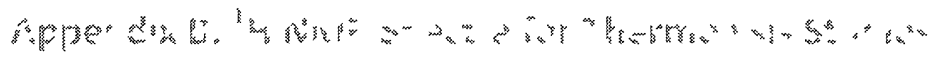

\section{Compound 1: [ $\left.\mathrm{Me}_{2} \mathrm{NC}\left(\mathrm{N}^{\prime} \mathrm{Pr}\right)_{2}\right]_{2} \mathrm{AlH}$}

Room Temperature

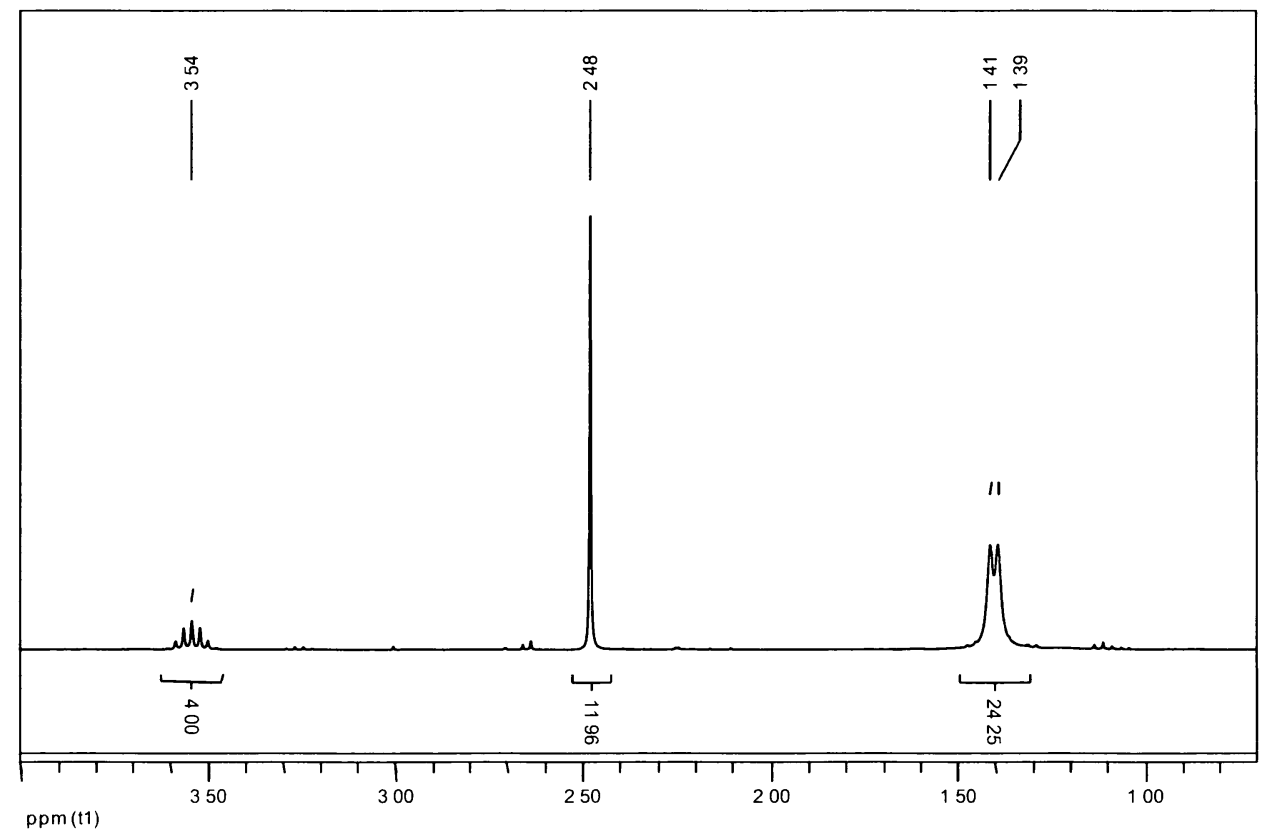

$56^{\circ} \mathrm{C}$ after 310 hours:

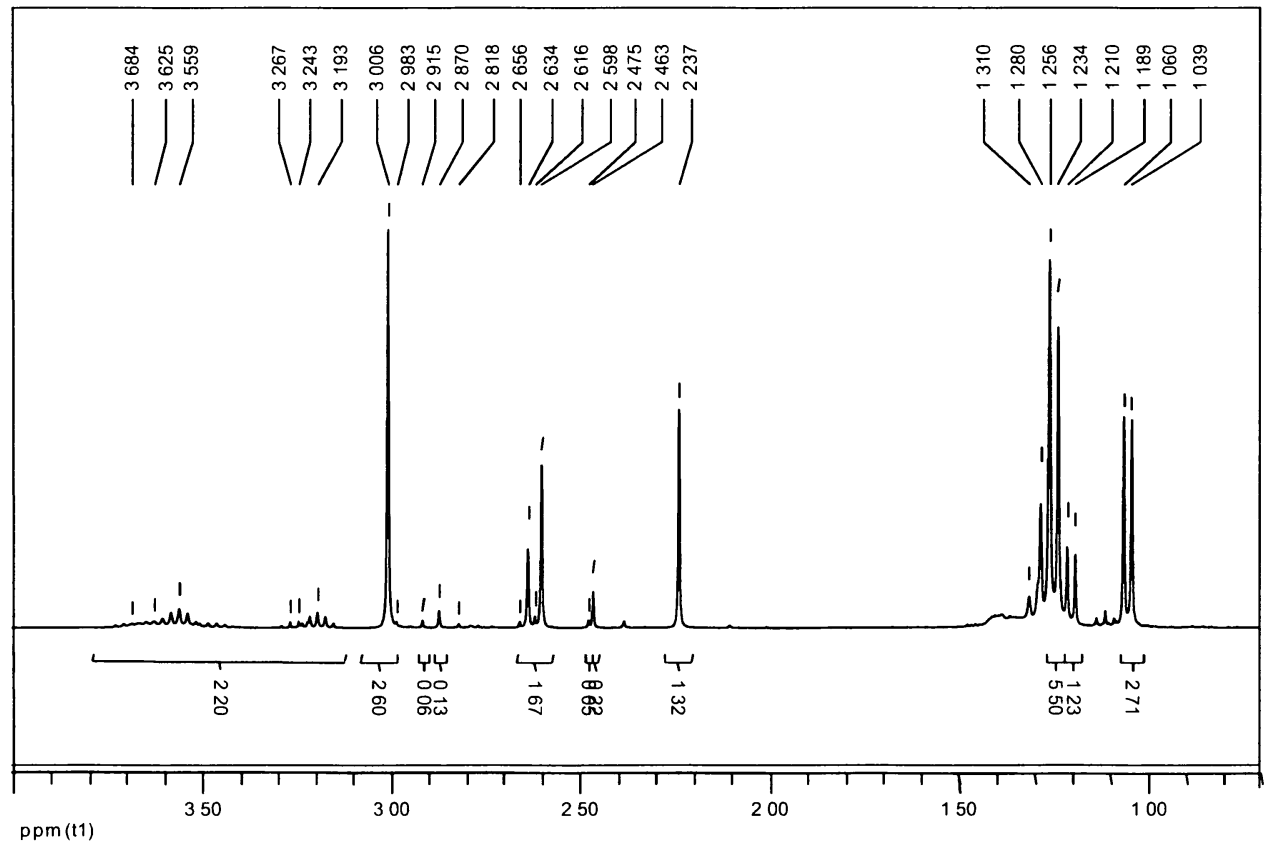




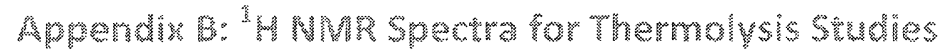

\section{Compound 2: $\left[\mathrm{Et}_{2} \mathrm{NC}\left(\mathrm{N}^{\mathrm{i}} \mathrm{Pr}\right)_{2}\right]_{2} \mathrm{AlH}$}

\section{Room Temperature:}

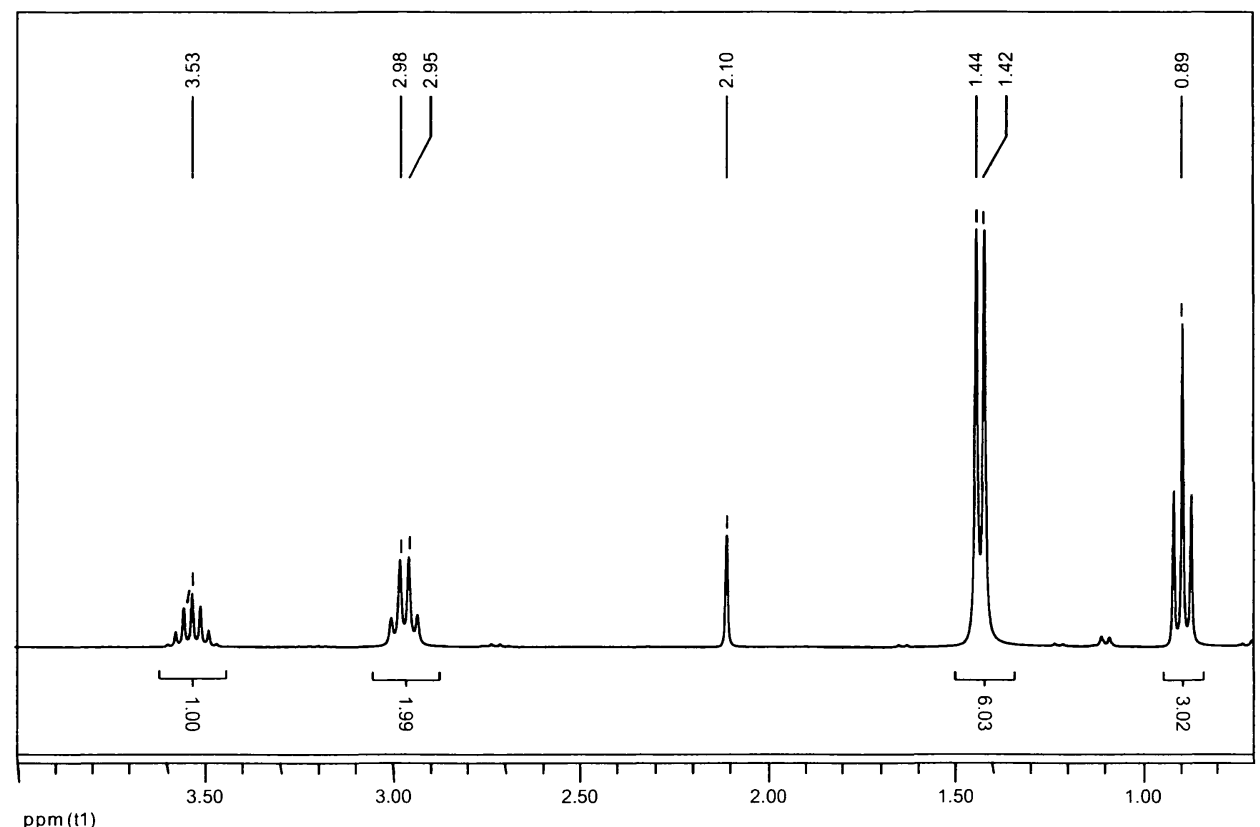

$56^{\circ} \mathrm{C}$ after 310 hours:

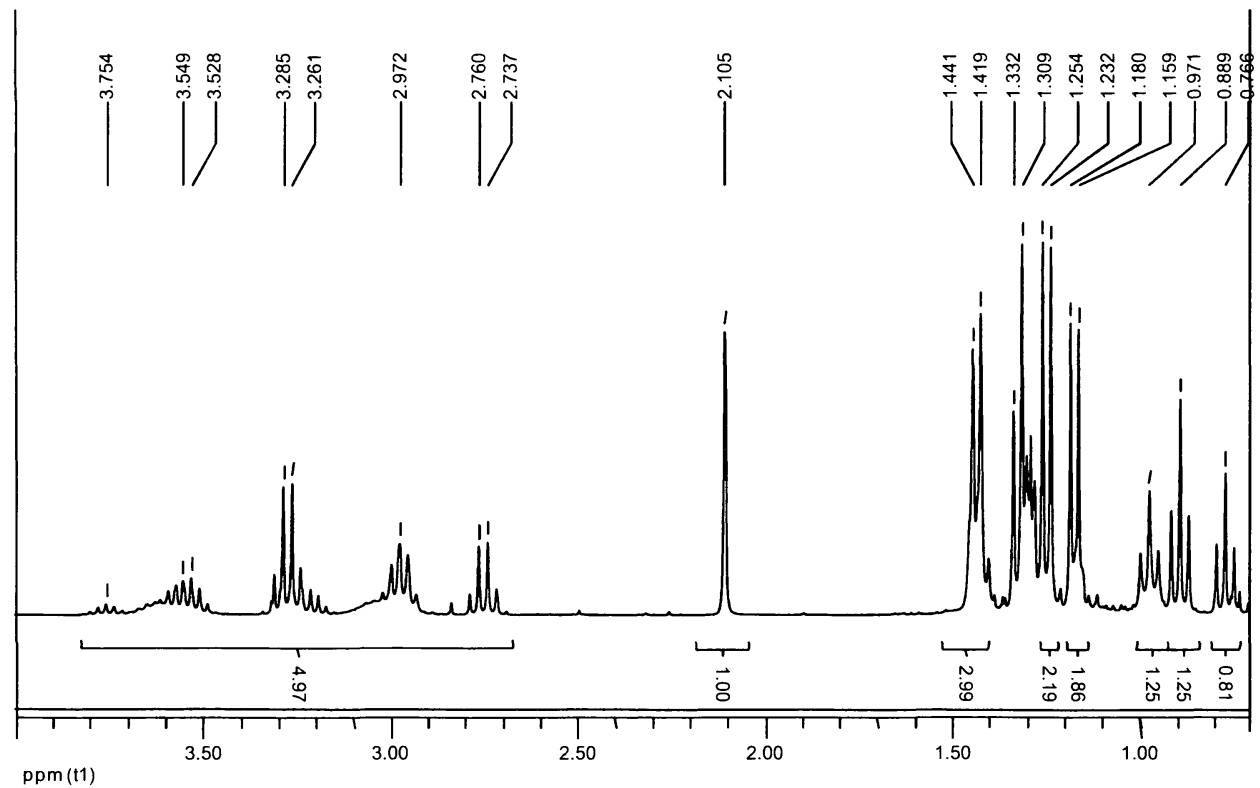




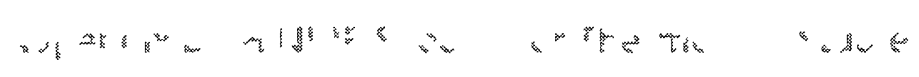

\section{Compound 3: ['PrHNC(N'Pr) $\left.)_{2}\right]_{2} \mathrm{AlH}$}

\section{Room Temperature}

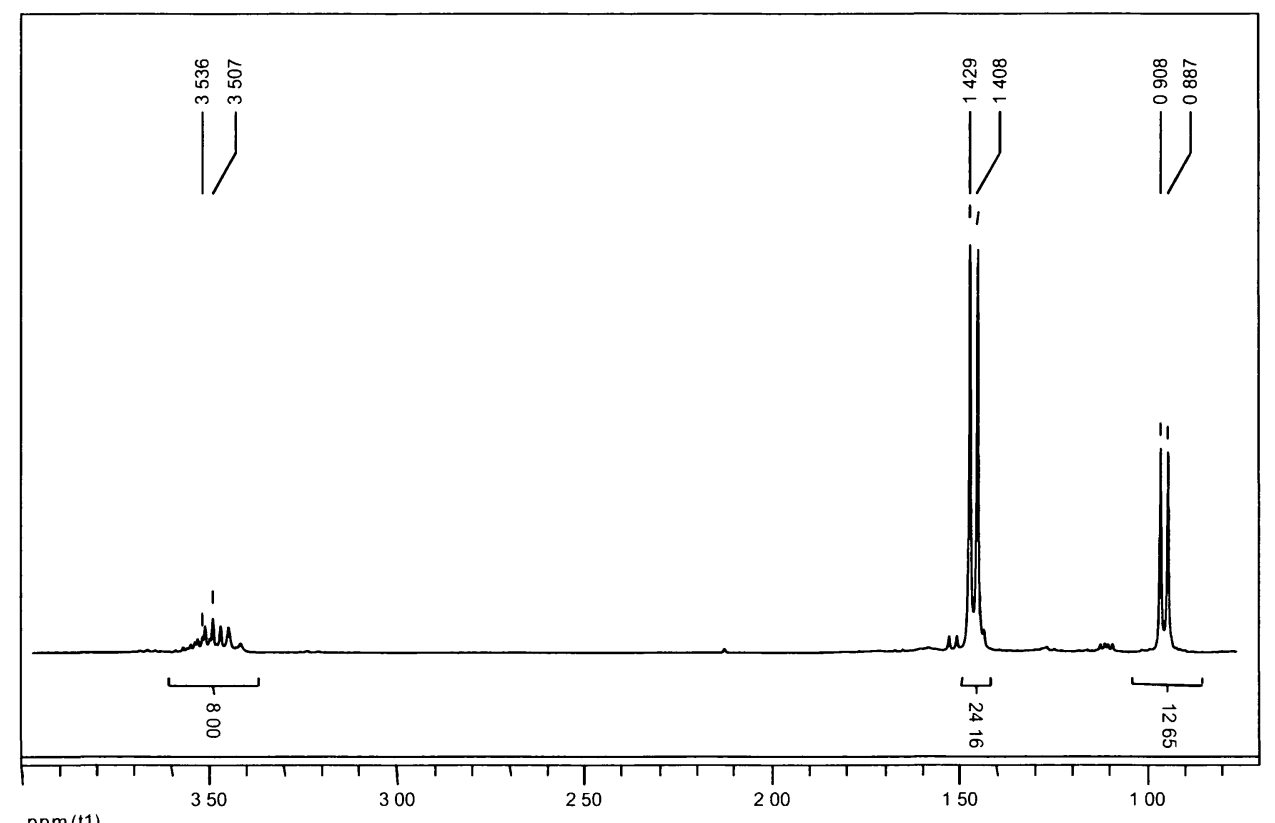

ppm(t1)

\section{$65^{\circ} \mathrm{C}$ after 148 hours}

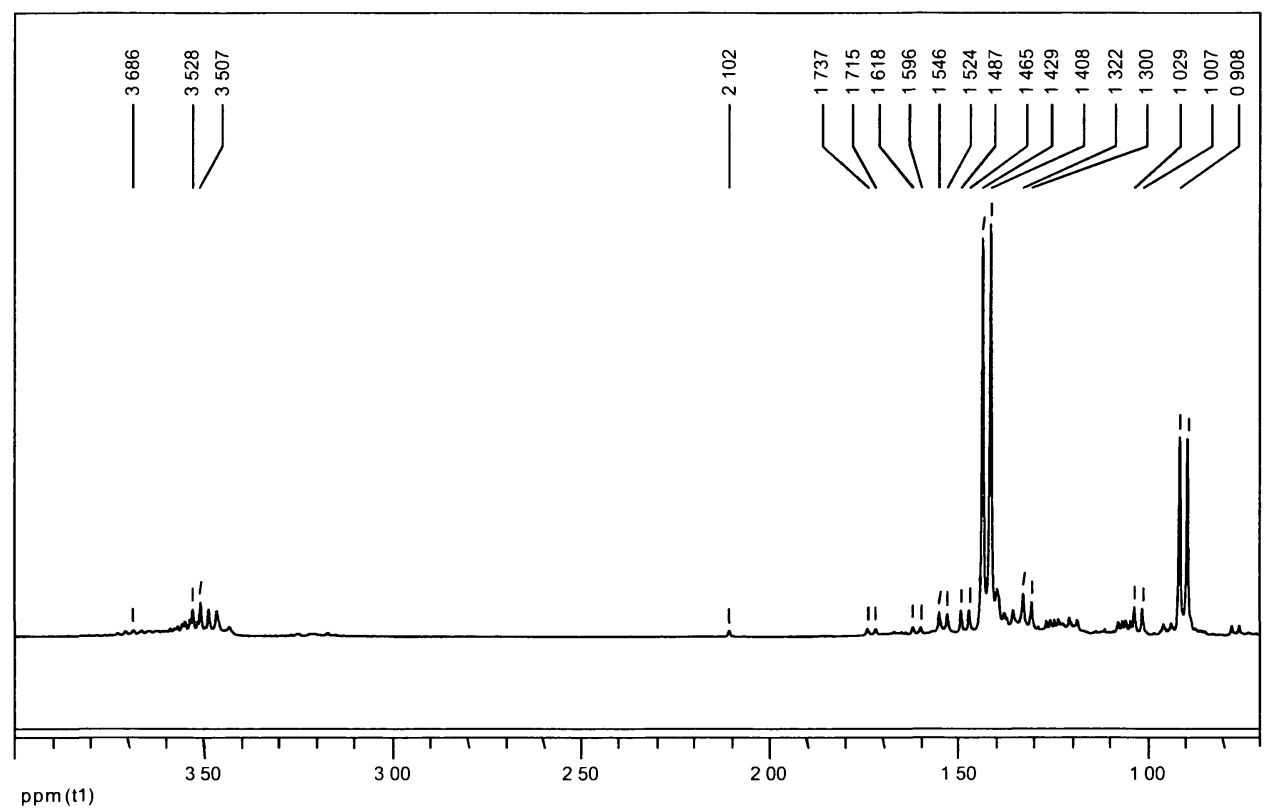




\section{Compound 4: $\left[{ }^{\mathrm{i}} \mathrm{Pr}_{2} \mathrm{NC}\left(\mathrm{N}^{\mathrm{i}} \mathrm{Pr}\right)_{2}\right]_{2} \mathrm{AlH}$}

Room Temperature:

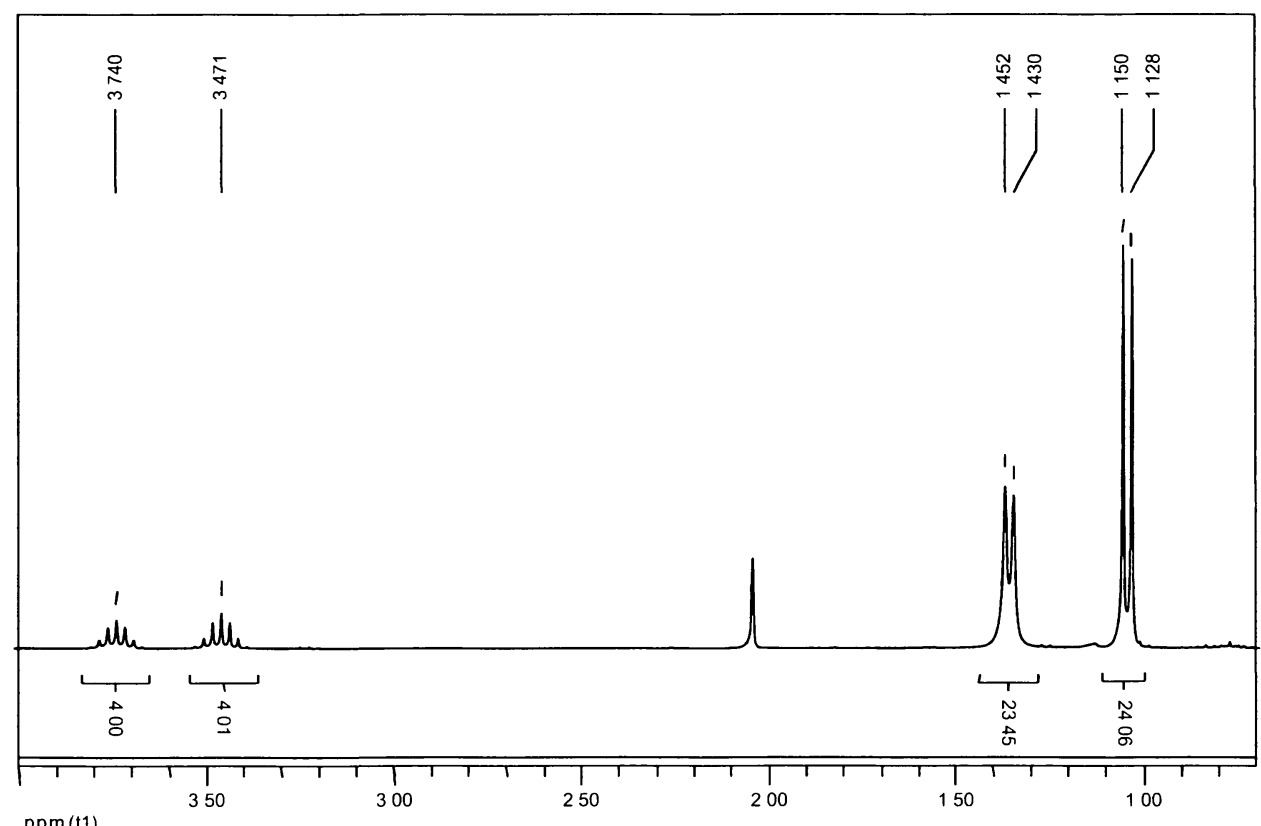

$\operatorname{ppm}(\mathrm{t} 1)$

$65^{\circ} \mathrm{C}$ after 148 hours:

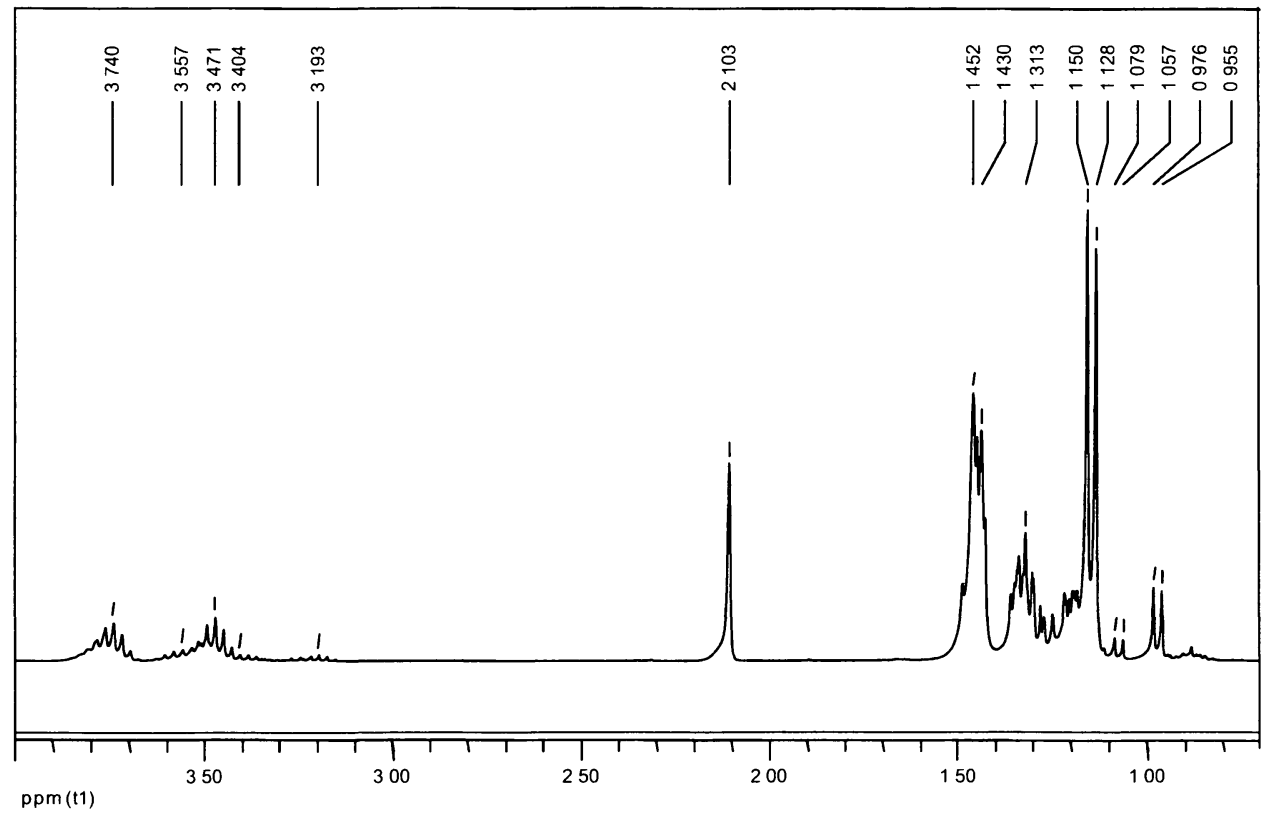


6.

\section{Compound 5: [MeC(N'Pr $\left.)_{2}\right]_{2} \mathrm{AlH}$}

Room Temperature:

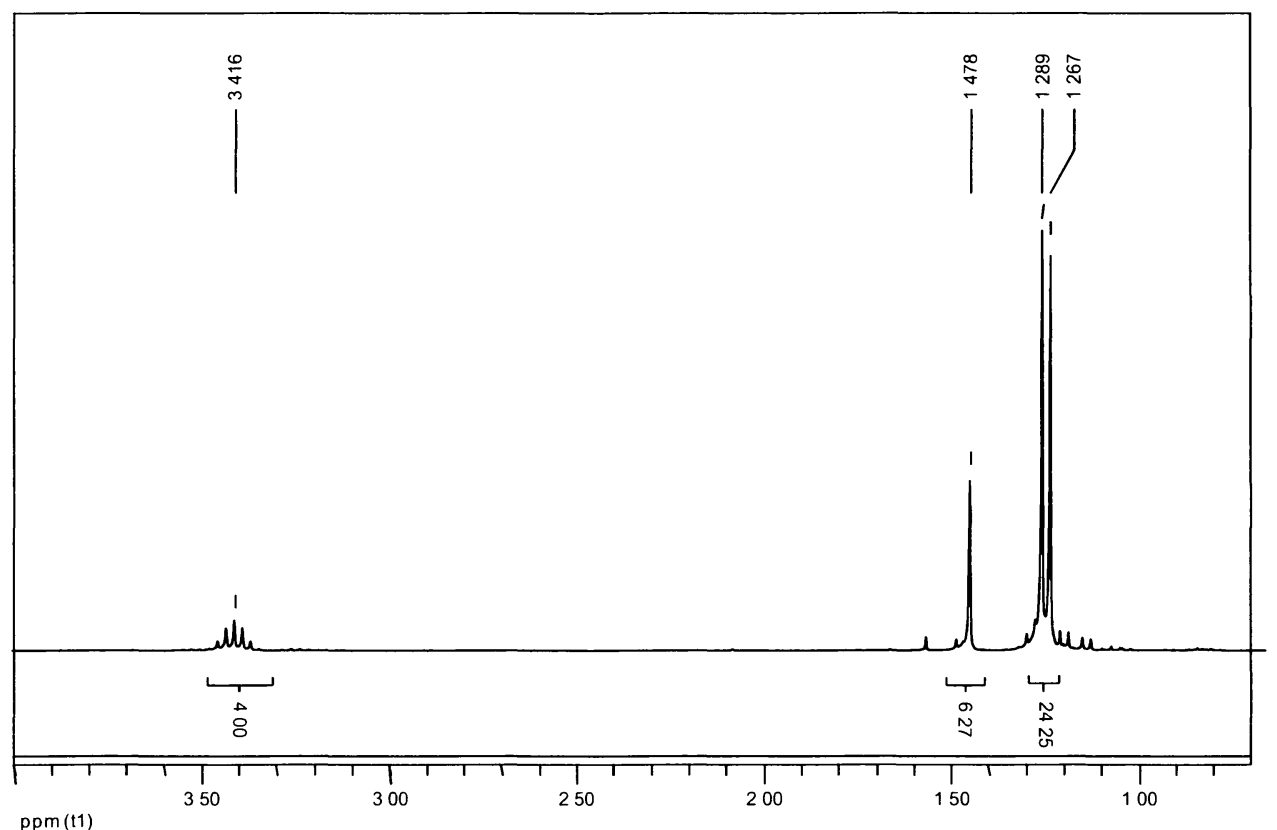

$65^{\circ} \mathrm{C}$ after 105 hours:

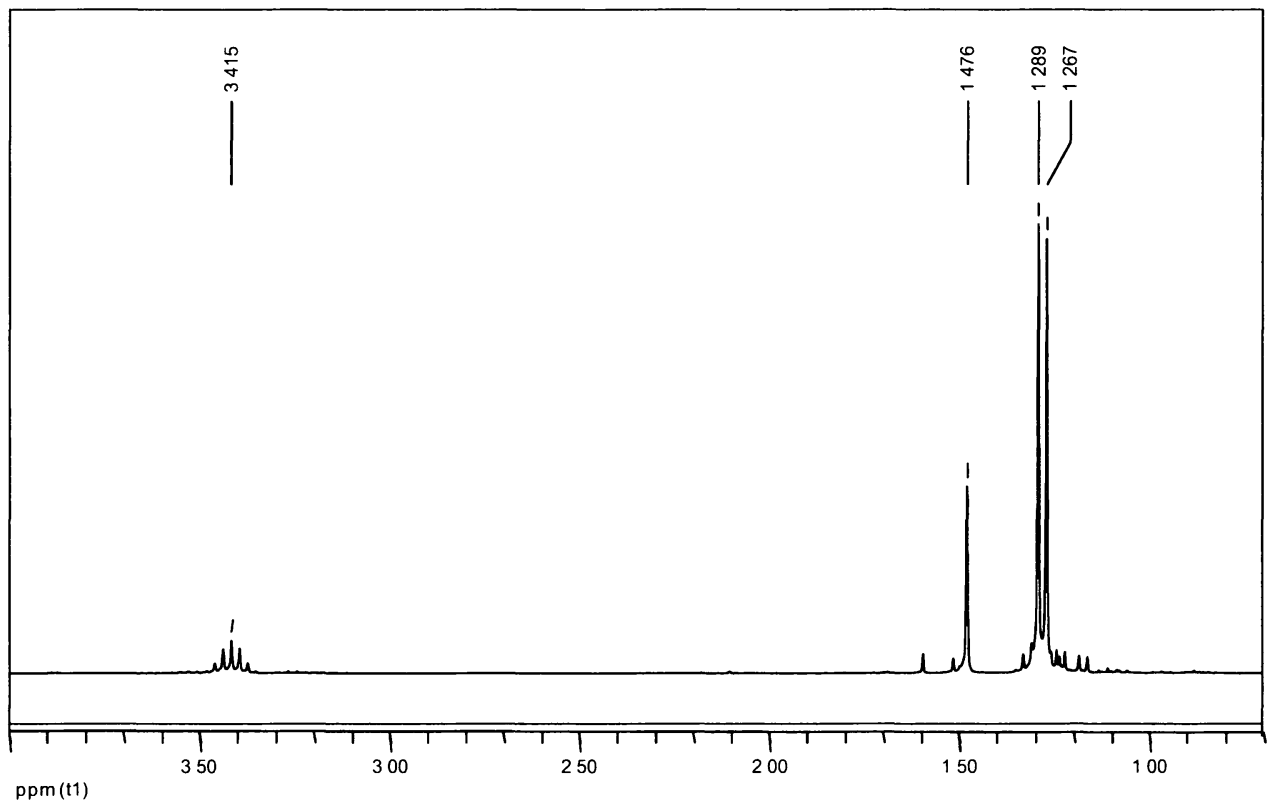




\section{Appendix C: Deposition Parameters}

Schematic diagram of hot-walled ALD reactor...........................................................

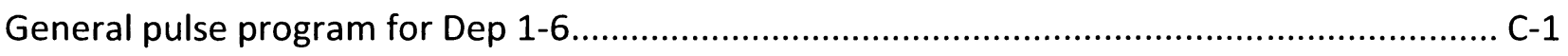

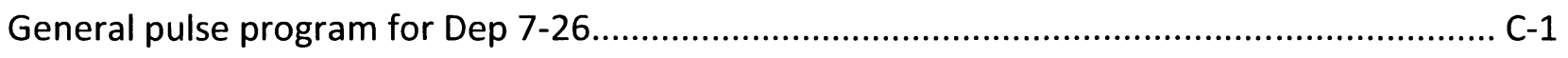

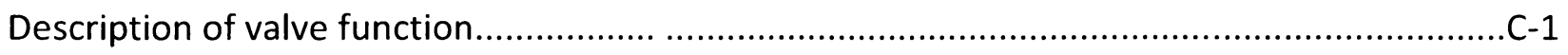

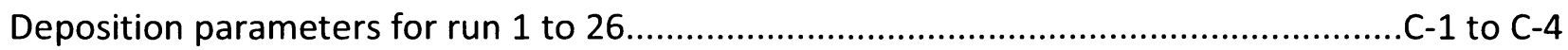




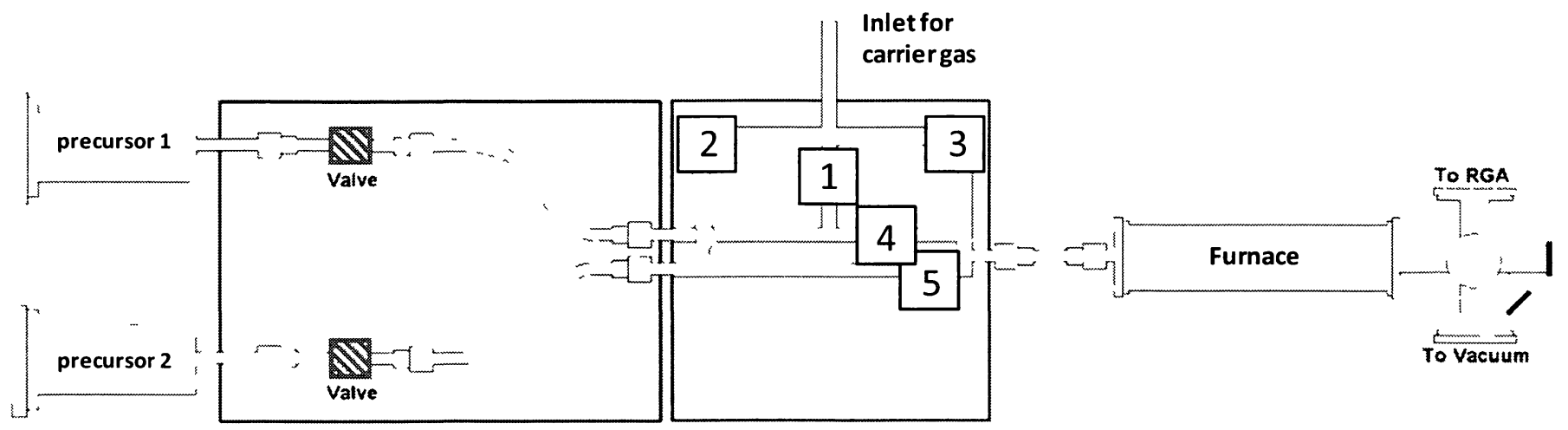

Figure 46. Schematic diagram of hot-walled ALD reactor

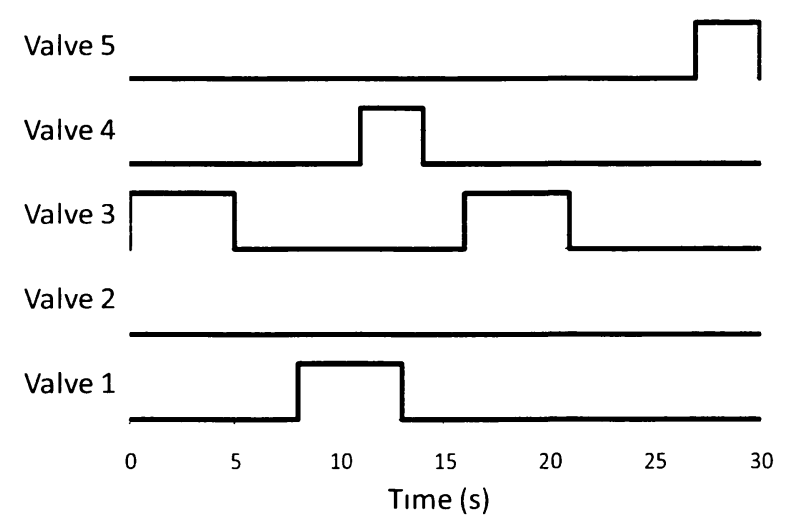

Figure 47. General pulse program for Dep 1-6

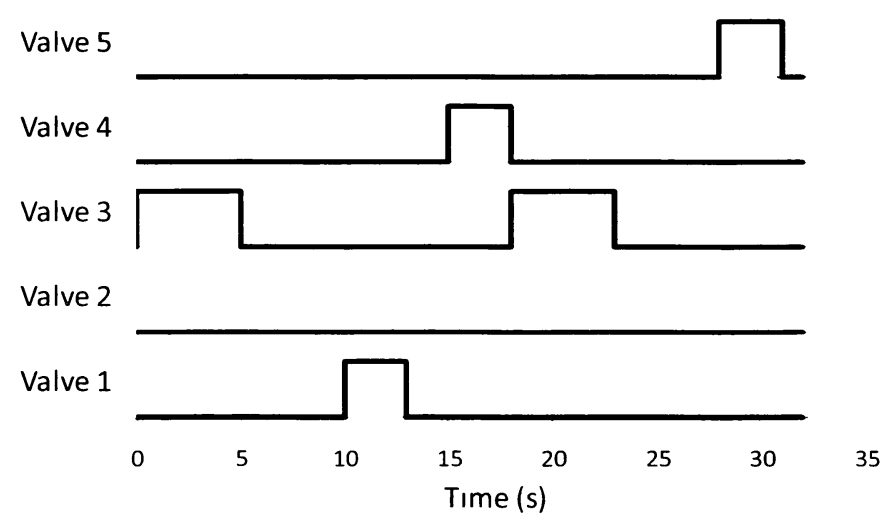

Figure 48. General pulse program for Dep 7-26

\section{Description of valve function:}

Valve 1: Introduces nitrogen carrier gas into precursor bubbler

Valve 2: Introduces nitrogen carrier gas into secondary precursor line (N/A for this work)

Valve 3: Introduces nitrogen carrier gas into deposition furnace

Valve 4: Opens precursor bubbler to deposition furnace

Valve 5: Opens secondary precursor line to deposition furnace 


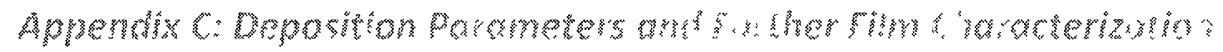

Table 11. Deposition run parameters

\begin{tabular}{|c|c|c|c|c|c|c|c|c|c|c|}
\hline & & Dep 1 & Dep 2 & Dep 3 & Dep 4 & Dep 5 & Dep 6 & Dep 7 & Dep 8 & Dep 9 \\
\hline \multirow{5}{*}{ 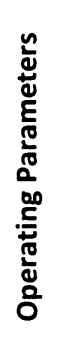 } & Bubbler $\mathrm{T}\left({ }^{\circ} \mathrm{C}\right)$ & 80 & 95 & 95 & 95 & 105 & 95 & 95 & 95 & 95 \\
\hline & Furnace $\mathrm{T}\left({ }^{\circ} \mathrm{C}\right)$ & 250 & 250 & 275 & 300 & 350 & 250 & 250 & 250 & 300 \\
\hline & Base pressure (mTorr) & --- & --- & --- & --- & --- & 70 & 78 & --- & 76 \\
\hline & $\begin{array}{l}\text { System pressure during } \\
\text { H2 flow (mTorr) }\end{array}$ & very high & very high & very high & very high & very high & very high & 470 & 470 & 475 \\
\hline & \# of cycles & 500 & 500 & 800 & 775 & 820 & 250 & 250 & 250 & 500 \\
\hline \multirow{9}{*}{ 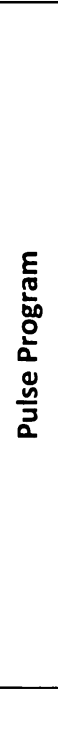 } & 3 close & 1000 & 1000 & 1000 & 1000 & 1000 & 1000 & 1000 & 1000 & 1000 \\
\hline & 1 open & 1600 & 1600 & 1600 & 1600 & 1600 & 1600 & 1600 & 2000 & 2000 \\
\hline & 1 close & 2600 & 2600 & 2600 & 2600 & 2600 & 2000 & 2000 & 2600 & 2600 \\
\hline & 4 open & 2200 & 2200 & 2200 & 2200 & 2200 & 2200 & 2200 & 3000 & 3000 \\
\hline & 4 close & 2800 & 2800 & 2800 & 2800 & 2800 & 2800 & 2800 & 3600 & 3600 \\
\hline & 5 open & 5400 & 5400 & 5400 & 5400 & 5400 & 4800 & 4800 & 5600 & 5600 \\
\hline & 5 close & 6000 & 6000 & 6000 & 6000 & 6000 & 5000 & 5400 & 6200 & 6200 \\
\hline & Delay & 0 & 0 & 0 & 0 & 0 & 0 & 200 & 200 & 200 \\
\hline & Total run time (h) & 4.17 & 4.17 & 6.67 & 6.46 & 6.83 & 1.74 & 1.94 & 2.22 & 4.44 \\
\hline
\end{tabular}




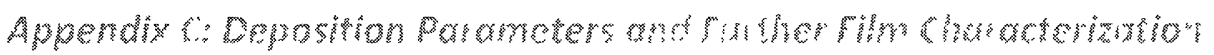

Table 2. Deposition run parameters (Continued)

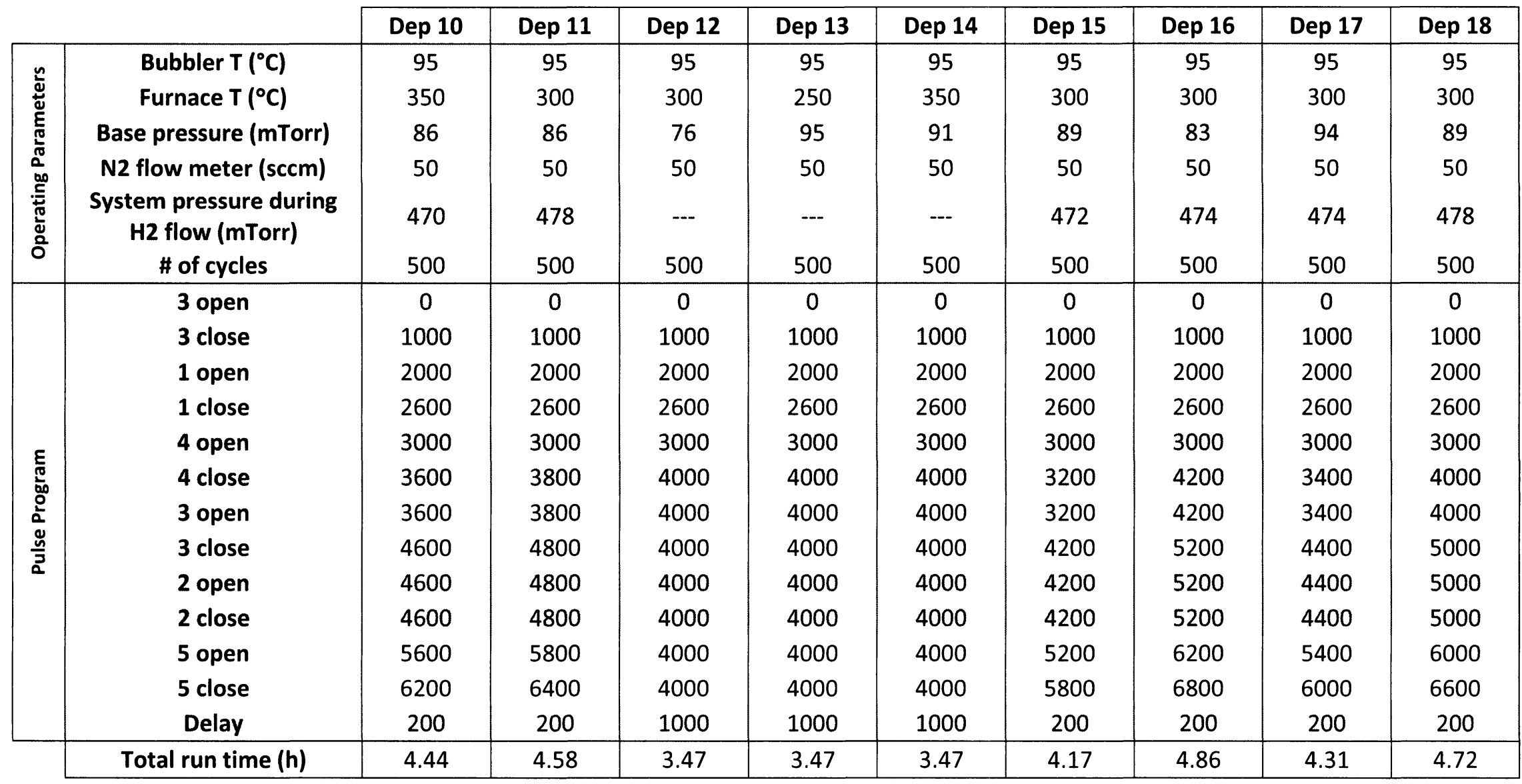




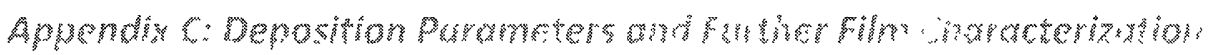

Table 3. Deposition run parameters (Continued)

\begin{tabular}{|c|c|c|c|c|c|c|c|c|c|}
\hline & & Dep 19 & Dep 20 & Dep 21 & Dep 22 & Dep 23 & Dep 24 & Dep 25 & Dep26 \\
\hline \multirow{6}{*}{ 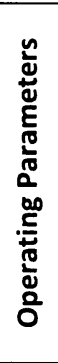 } & Bubbler $\mathrm{T}\left({ }^{\circ} \mathrm{C}\right)$ & 95 & 95 & 95 & 95 & 95 & 95 & 95 & 95 \\
\hline & Furnace $\mathrm{T}\left({ }^{\circ} \mathrm{C}\right)$ & 250 & 250 & 250 & 250 & 250 & 250 & 250 & 250 \\
\hline & Base pressure (mTorr) & 85 & 87 & 84 & 88 & 94 & 98 & 91 & 82 \\
\hline & N2 flow meter (sccm) & 50 & 50 & 50 & 50 & 50 & 50 & 50 & 50 \\
\hline & $\begin{array}{l}\text { System pressure during } \\
\text { H2 flow (mTorr) }\end{array}$ & 445 & 433 & 447 & 440 & 473 & 434 & 441 & 475 \\
\hline & \# of cycles & 400 & 400 & 350 & 300 & 400 & 400 & 300 & 2258 \\
\hline \multirow{14}{*}{ 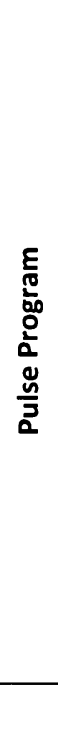 } & 3 open & 0 & 0 & 0 & 0 & 0 & 0 & 0 & 0 \\
\hline & 3 close & 1000 & 1000 & 1000 & 1000 & 1000 & 1000 & 1000 & 1000 \\
\hline & 1 open & 2000 & 2000 & 2000 & 2000 & 2000 & 2000 & 2000 & 2000 \\
\hline & 1 close & 2600 & 2600 & 2600 & 2600 & 2600 & 2600 & 2600 & 2600 \\
\hline & 4 open & 3000 & 3000 & 3000 & 3000 & 3000 & 3000 & 3000 & 3000 \\
\hline & 4 close & 3400 & 3600 & 3800 & 4000 & 3200 & 3100 & 4000 & 4200 \\
\hline & 3 open & 3400 & 3600 & 3800 & 4000 & 3200 & 3100 & 4000 & 4200 \\
\hline & 3 close & 4400 & 4600 & 4800 & 5000 & 4200 & 4100 & 5000 & 5200 \\
\hline & 2 open & 4400 & 4600 & 4800 & 5000 & 4200 & 4100 & 5000 & 5200 \\
\hline & 2 close & 4400 & 4600 & 4800 & 5000 & 4200 & 4100 & 5000 & 5200 \\
\hline & 5 open & 5400 & 5600 & 5800 & 6000 & 5200 & 5100 & 6000 & 6200 \\
\hline & 5 close & 6000 & 6200 & 6400 & 6600 & 5800 & 5700 & 6600 & 6800 \\
\hline & Delay & 200 & 200 & 200 & 200 & 200 & 200 & 200 & 200 \\
\hline & Total run time (h) & 3.44 & 3.56 & 3.21 & 2.83 & 3.33 & 3.28 & 2.83 & 21.95 \\
\hline
\end{tabular}

